TESIS DE DOCTORADO EN GEOFÍSICA

\title{
IMPLEMENTACIÓN DE LA RED DE ESTACIONES SISMOLÓGICAS DE LA UNIVERSIDAD NACIONAL DE LA PLATA. APLICACIONES.
}

NORA CRISTINA SABBIONE

DIRECTORA: Dra. ANA MARIA OSELLA

SUBDIRECTOR: Ing. ROBERTO MARIO PINCIROLI

Noviembre de 2004.

DEPARTAMENTO DE SISMOLOGÍA E INFORMACIÓN METEOROLÓGICA FACULTAD DE CIENCIAS ASTRONÓMICAS Y GEOFÍSICAS UNIVERSIDAD NACIONAL DE LA PLATA 
a Federico, Erica, Ernesto, Rodolfo, Fernando, Francisco, Gerardo, Dory y César quienes me apoyaron y permitieron la concreción de este trabajo. 


\section{Agradecimientos}

Sin lugar a dudas, deseo expresar que no hubiera podido llevar a cabo este trabajo sin la guía de la Dra. Ana María Osella. Ella además de haber cumplido con responsabilidad y dedicación la dirección de esta Tesis, guiándome, aconsejándome y aportando valiosas correcciones, ha excedido sobradamente su función, para acompañarme en lo personal y afectivo durante este proceso.

A la memoria del Ing. Roberto Mario Pinciroli, quien durante los dos primeros años de este trabajo y por un período de diez años en Proyectos conjuntos me siguió y ayudó a resolver todos los temas. A él le estaré siempre agradecida.

La instalación y operación de las estaciones sismológicas digitales mencionadas en este trabajo son el resultado del esfuerzo de un grupo de personas entre las que cabe destacar principalmente a quienes se ocupan de la atención y operación de las mismas: el personal del Departamento de Sismología de FCAGLP, el personal del Observatorio Geofísico de Trelew y el personal de la Estación Astronómica Rio Grande. Además, la instalación de la estación DSPA ha sido posible gracias a la familia Larminat, dueños de la Estancia Despedida, que cedieron el lugar para el emplazamiento de la estación y permanentemente nos dan su ayuda.

Deseo manifestar también mi agradecimiento a todos aquellos que en algún momento me alentaron; en particular a la Geof. María Laura Rosa, al Ing. Juan S. Carmona y al Lic. Ruben E. Martínez. 


\section{ÍNDICE}

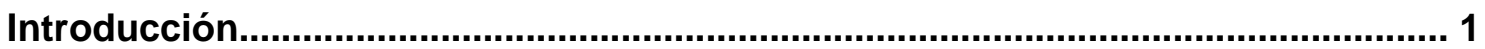

1.- Capítulo 1 : Introducción a la Sismometría.................................................... 4

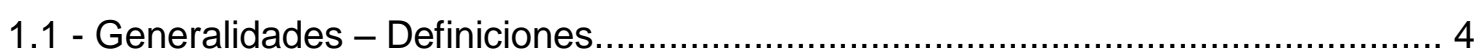

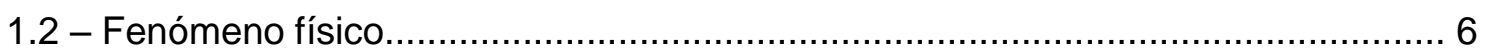

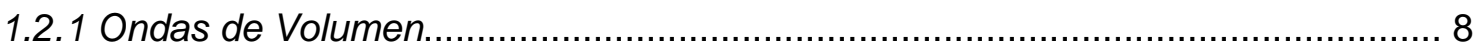

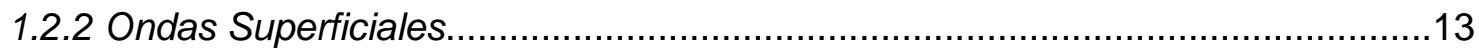

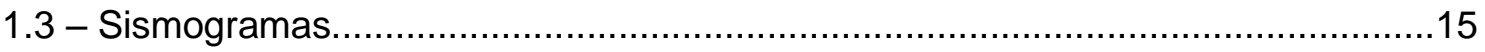

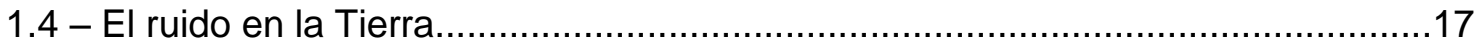

2.-Capítulo 2 : Estación sismológica moderna........................................................ 21

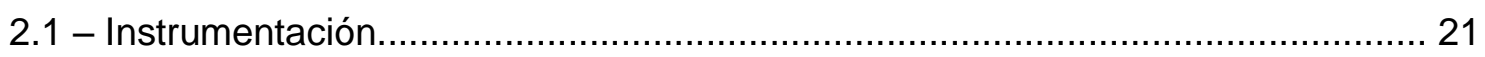

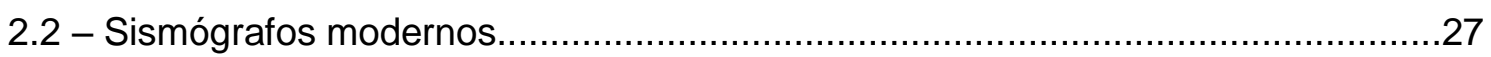

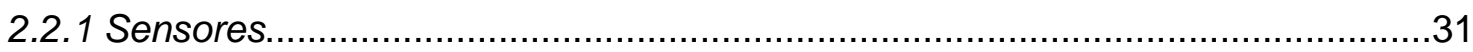

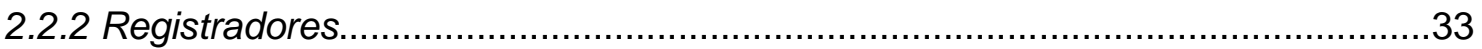

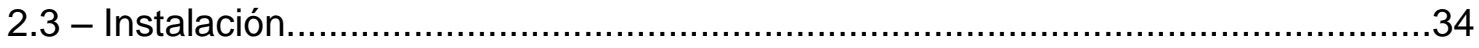

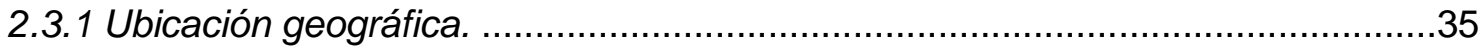

2.3.2 Selección del sitio y campaña de ruido sísmico. ..............................................36

2.3.3 Instalación de la estación sismológica. ...............................................................38

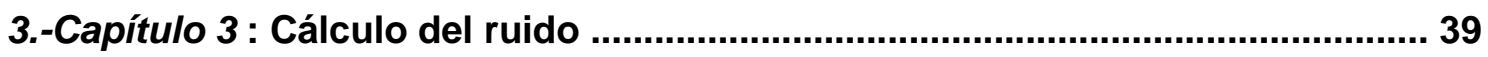

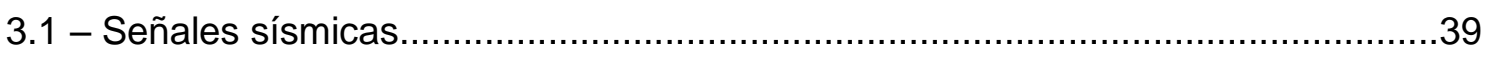

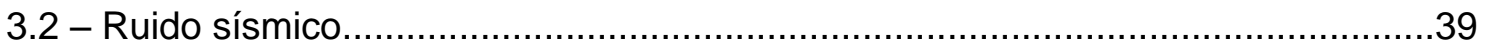

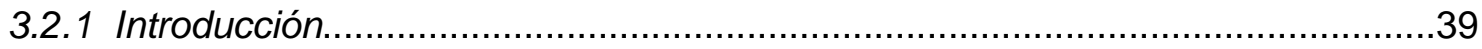

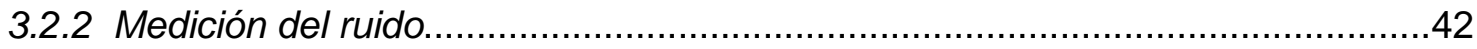

3.2.3 Relación entre mediciones de densidad de potencia espectral y de amplitud.....43

4.-Capítulo 4: Estación Sismológica LPAD, La Plata Digital................................ 47

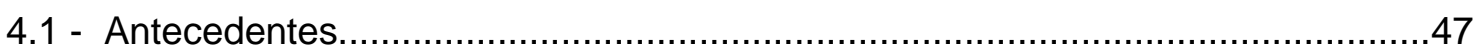

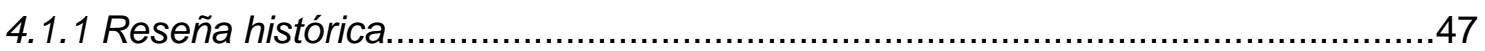

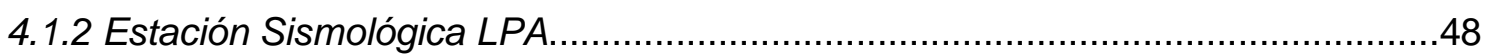

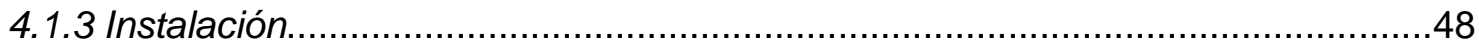

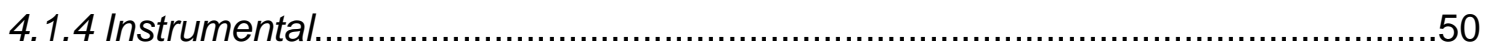




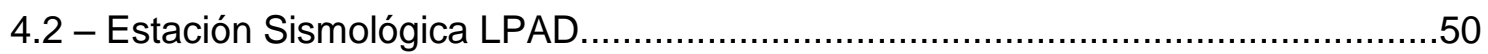

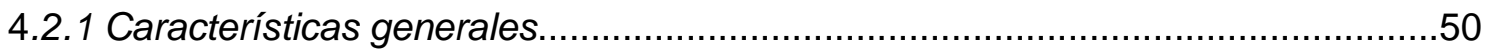

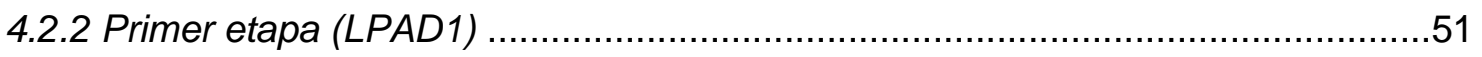

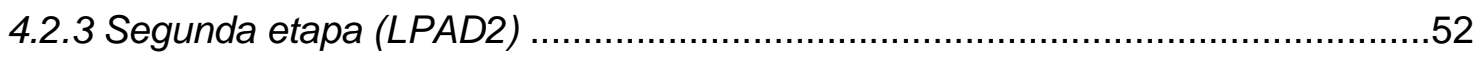

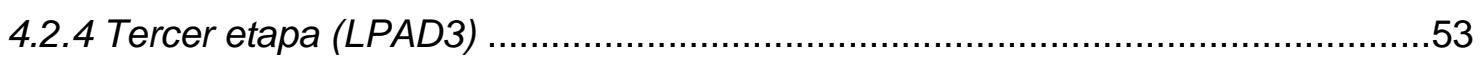

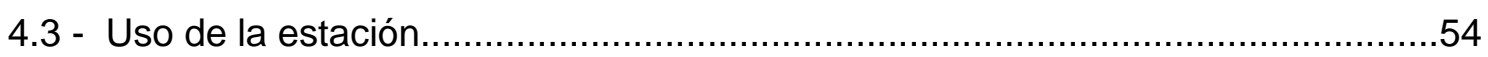

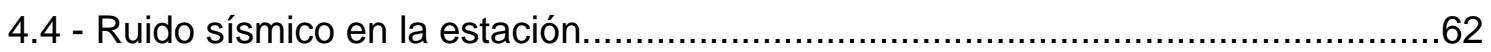

4.4.1 Aspectos del ruido sísmico en aceleración..........................................................62

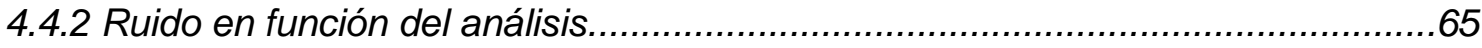

de densidad espectral de potencia en aceleración.

5.- Capítulo 5: Estación sismológica TRWA. Trelew Argentina. ............................67

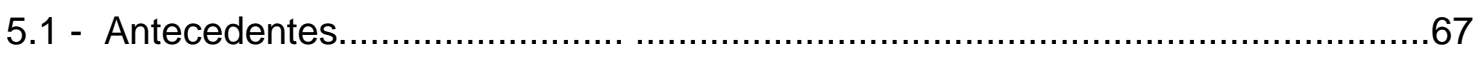

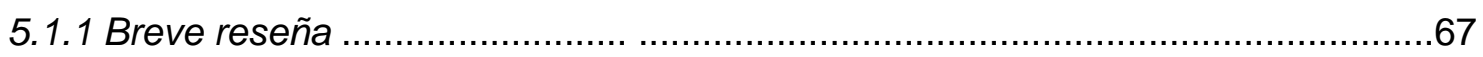

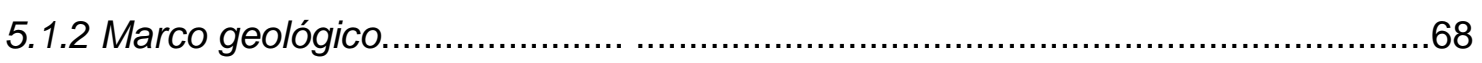

5.1.3 Instalación de instrumental de corto período........................................................69

5.1.4 Análisis del ruido y comparación con La Plata......................................................72

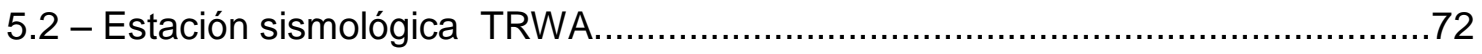

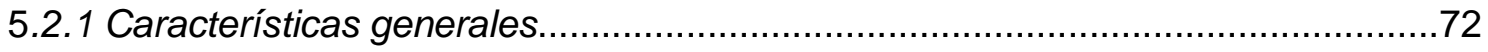

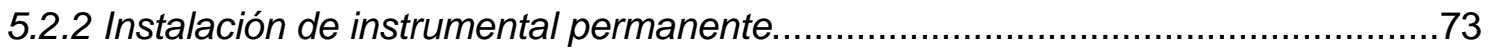

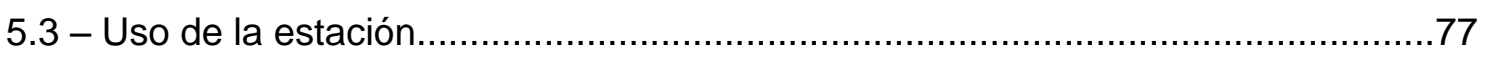

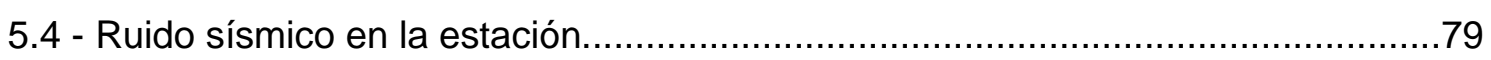

5.4.1 Aspectos del ruido sísmico en aceleración.......................................................79

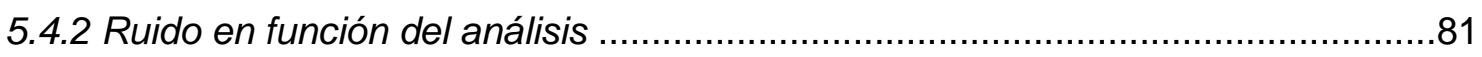

de densidad espectral de potencia en aceleración.

6.- Capítulo 6: Estación Sismológica DSPA. Eª Despedida, Argentina....................82

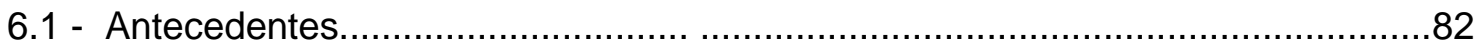

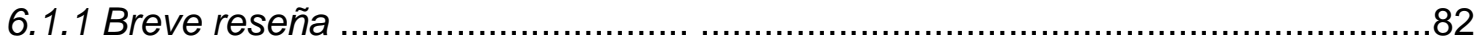

6.1.2 Marco geológico y geodinámico................................................................. 83

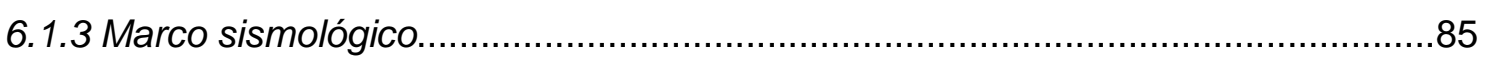

6.2 - Estación sismológica DSPA. 1era etapa...................................................... 86

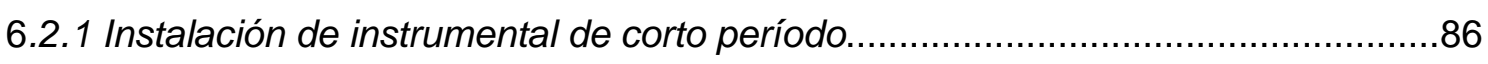

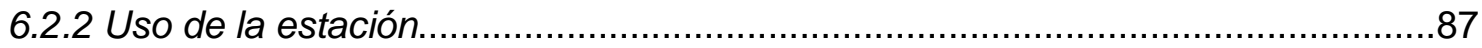

6.2.3 Análisis del ruido en velocidad con instrumental de corto período........................89 


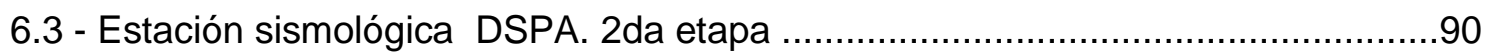

6.3.1 - Instalación de instrumental banda ancha ........................................................90

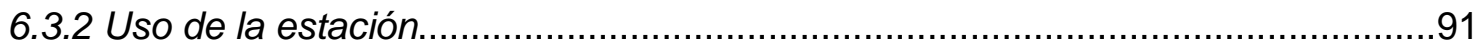

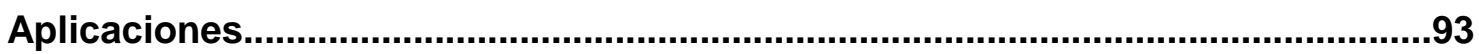

7.- Capítulo 7: Determinación e inversión de curvas de dispersión de ondas Rayleigh en distintas trayectorias hacia La Plata (Pcia. de Bs.As.)......................94

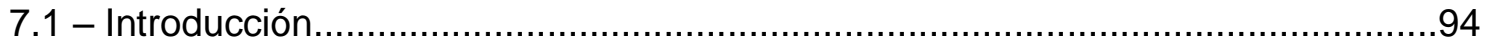

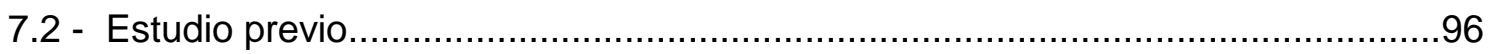

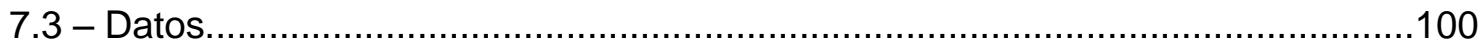

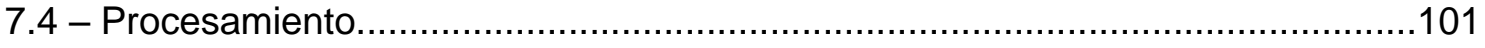

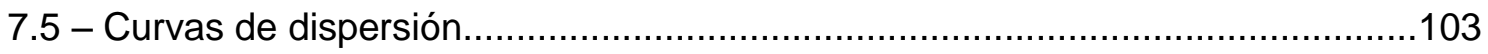

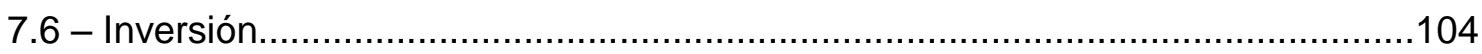

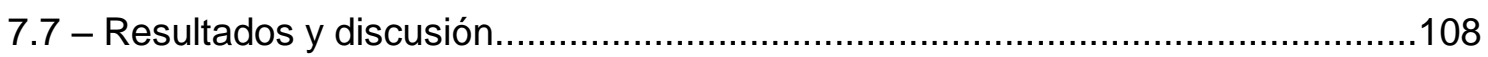

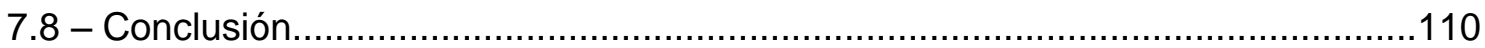

8. Capítulo 8 : El movimiento causado por los sismos de mayor magnitud de la zona central de Chile en edificios altos de Buenos Aires ..........................112

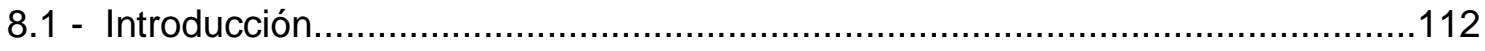

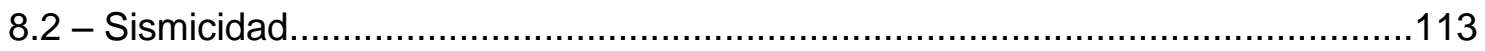

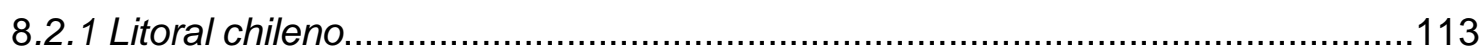

8.2.2 - Ciudad de Buenos Aires y alrededores........................................................117

8.3 - Seis edificios altos de la ciudad de Buenos Aires...........................................121

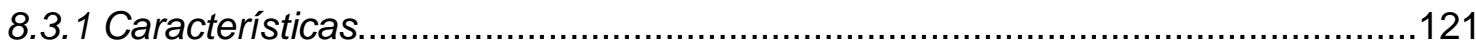

8.3.2 Periodos fundamentales de vibración horizontal.............................................123

8.4 - Efecto de sismos de mayor magnitud de la zona Central de Chile ...................124

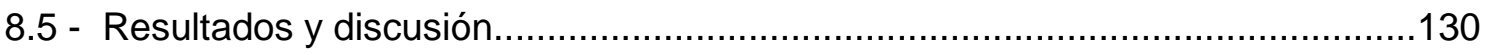

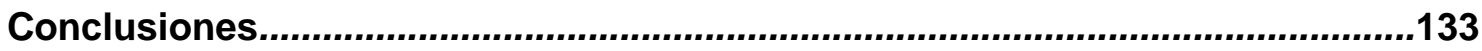

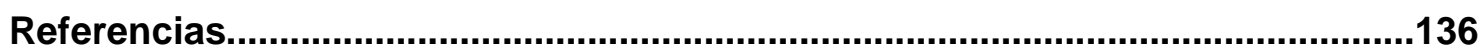




\section{INTRODUCCIÓN}

La estructura sísmica de la corteza y manto superior que yace debajo de América del Sur aún se mantiene pobremente caracterizada, particularmente en comparación con otras regiones, tales como Norteamérica y Eurasia. Esto se debe a que Sudamérica no cuenta con una red de sismógrafos permanente equivalente a la del hemisferio Norte y esa falta de instrumentación lleva a una subestimación de su sismicidad. Por otro lado, al no contar con una buena distribución de estaciones, los mecanismos de foco no pueden ser determinados en cualquier sitio para el caso de bajas magnitudes, lo cual dificulta avanzar en el conocimiento de los procesos dinámicos y de la estructura terrestre para esas regiones. Obviamente, si se contara con un número mayor de estaciones y con buena distribución, eventos de menor magnitud podrían ser detectados y analizados.

La Red Nacional de Estaciones Sismológicas (INPRES) de la República Argentina está conformada por cincuenta estaciones. Hay diecisiete estaciones en el noroeste, veintisiete en el centro y oeste, tres al noreste y una en Patagonia y Tierra del Fuego. De todas estas estaciones sólo cinco corresponden a estaciones digitales de banda ancha.

Claramente queda evidenciada la necesidad de lograr una mejor cobertura de nuestro territorio nacional, especialmente en la región este-sudeste. Con el fin de suplir esa falencia en esta zona, me he planteado como objetivo a largo plazo la implementación de una red digital con instrumental de última generación que abarque la zona patagónica.

En este contexto, realicé como primer paso la actualización de la Estación sismológica de La Plata. Esta estación data de 1962 y ha formado parte de la red mundial World Wide Standard Seismographic Network (WWSSN) hasta el año 1996. Esta estación analógica había quedado desactualizada, de modo que su modernización era necesaria para mantener su contribución dentro del contexto internacional.

Como segundo paso y continuando con el objetivo planteado, instalé dos nuevas estaciones en Trelew (Chubut) y Despedida (Tierra del Fuego) lo cual ya permite una mínima cobertura de la zona.

La determinación del sitio adecuado para la instalación de una nueva estación requiere de un amplio estudio de prefactibilidad. El criterio más importante para la 
selección de un buen lugar es el bajo ruido sísmico, pero también hay que considerar que en algunos casos este criterio debe ser adecuado para asegurar una buena cobertura. Otros factores técnicos importantes en la definición del lugar son la accesibilidad y la posibilidad de contar con electricidad, mientras que el factor más importante como especificación no técnica, es el soporte local disponible.

Este proceso requiere un exhaustivo análisis para optimizar todos estos parámetros, tratando de mantener la más alta calidad y que sea compatible con los recursos económicos disponibles. Por eso la necesidad de los estudios de calidad de los registros a fin de garantizar que los datos obtenidos satisfagan los parámetros necesarios para su incorporación a bases de datos internacionales como para confeccionar una base de datos local.

Teniendo en cuenta estos criterios, en este trabajo describo los pasos seguidos para la actualización de la estación de La Plata y para la instalación de las nuevas estaciones, los distintos tipos de instrumentos utilizados y los estudios de ruido realizados a tal efecto. Luego presento el nivel de ruido en densidad de potencia espectral de la aceleración y lo comparo con las curvas de nivel de ruido tomadas como standards actualmente, dando cuenta de la calidad de los registros que se obtienen.

Como ejemplo de la potencialidad y del procesamiento de los datos digitales que se están adquiriendo, presento luego dos estudios concretos, en este caso, con datos de la estación sismológica de La Plata. Las Aplicaciones desarrolladas en la segunda parte de este Trabajo de Tesis, son aportes al conocimiento de la tectónica y a la ingeniería sismorresistente en nuestro territorio nacional.

En una primera aplicación, presento el análisis de dispersión de ondas Rayleigh a efectos de investigar las propiedades físicas del sistema litósfera-astenósfera en la región de las Sierras Pampeanas. A tal efecto realizo la inversión de las curvas de dispersión obtenidas y defino perfiles de velocidad de corteza y manto superior. Previo a realizar este estudio he analizado otras trayectorias continentales hacia La Plata a fin de comprobar que los datos de nuestra estación resultaban útiles para obtener curvas de dispersión que reflejen claramente las distintas trayectorias entre evento y estación.

Como segunda aplicación, presento la determinación de los valores de aceleración a los que han sido sometidos los edificios más altos de la ciudad de Buenos Aires, al momento de estar excitados por las ondas sísmicas generadas en ocasión de sismos en el litoral chileno. La ciudad de Buenos Aires, con una población 
metropolitana de cerca de 10 millones de habitantes, está situada en el este de la gran llanura formada a partir de potentes capas de sedimentos aluviales que constituyen la pampa argentina. La actividad sísmica del área que la circunda es baja; sin embargo, la historia sísmica del oeste argentino así como de la costa oeste de América del Sur muestran que en cada centuria ocurren varios eventos sísmicos de magnitud mayor que $\mathrm{Ms}=8$, que pueden ser percibidos en esta zona. Teniendo en cuenta que actualmente los edificios más altos superan los cien metros de altura, es conveniente estimar las amplitudes del movimiento en los pisos superiores de estos edificios cuando son sacudidos por las ondas generadas por eventos sísmicos distantes y de gran magnitud.

Este trabajo consta de tres primeros capítulos donde se realiza una presentación teórica de los conceptos en Sismometría, Estación sismológica moderna y Cálculo de ruido, que luego serán utilizados; otros tres capítulos, cada uno de los cuales corresponde a una de las estaciones sismológicas digitales que se han actualizado e instalado en estos últimos años y finalmente dos capítulos con Aplicaciones del uso de datos digitales obtenidos en la estación sismológica La Plata. 


\section{Capítulo 1 \\ INTRODUCCIÓN A LA SISMOMETRÍA}

\section{1 - Generalidades - Definiciones}

La Tierra se compone, entre otros, de silicatos y de aleaciones de hierro con la importante propiedad que, en el amplio rango de condiciones de presión y temperatura existente en el planeta, los materiales responden casi elásticamente bajo la aplicación de fuerzas transitorias de pequeña magnitud, pero en forma viscosa bajo la aplicación de fuerzas de larga duración. Esta dependencia con el tiempo de las propiedades de los materiales significa que la Tierra "suena como una campana" cuando son aplicadas fuerzas de corta duración, tales como un rápido movimiento de rocas a través de una superficie de falla o detonación de una explosión, mientras que la convección global de flujo - fluido continuamente rehace la superficie y el interior del planeta a lo largo de una escala de tiempo geológico. La vibración mecánica resulta del comportamiento cuasi-elástico, que involucra excitación y propagación de ondas elásticas en el interior. Estas ondas son registradas por instrumentos que captan el movimiento del suelo, llamados sismómetros, hecho que permite el posterior análisis de las mismas.

La Sismología es el estudio de la generación, propagación y registro de ondas elásticas en la Tierra (y otros cuerpos celestes) y de las fuentes que las producen. Es una disciplina basada en lo observacional.

Tanto fuentes naturales como artificiales de energía deformacional pueden producir ondas sísmicas, perturbaciones elásticas que se expanden alejándose de la fuente como resultado de un esfuerzo transitorio de desbalanceo en las rocas.

Los procedimientos sismológicos proveen la mejor resolución de la estructura interna de la Tierra; esto se debe a que las ondas elásticas tienen la mayor penetración cuando ocurren terremotos tectónicos de magnitudes grandes y la física que las gobierna es sensible espacial y temporalmente al camino seguido por la energía. Estas propiedades proveen una resolución mucho mayor que la que se puede obtener a través de los campos eléctricos, gravitacional, magnético y térmico, ya que estos promedian grandes regiones y períodos temporales.

La información básica se encuentra en los sismogramas: registros del movimiento del suelo en función del tiempo.

En Sismología, debido a los diferentes tipos de fuentes así como a las distintas ondas sísmicas resultantes, se analiza un gran rango de las escalas. 
Los microsismos detectables tienen un momento sísmico (cantidad resultante del producto entre el área de la superficie de falla, el coeficiente de rigidez de la roca y el desplazamiento promedio en la falla) del orden de $10^{5} \mathrm{Nm}$ y grandes terremotos tienen momentos sísmicos tan grandes como $10^{23} \mathrm{Nm}$.

Las amplitudes del movimiento de las ondas sísmicas son directamente proporcionales a los momentos sísmicos, de modo que los desplazamientos de las ondas sísmicas desarrollan un enorme rango. El ruido natural del suelo, que es fuertemente dependiente de la frecuencia, tiene su límite en las menores amplitudes que se pueden medir, típicamente desplazamientos de $1 \mathrm{~nm}$ en $1 \mathrm{~Hz}$, mientras que los mayores desplazamientos son del orden de $1 \mathrm{~m}$. Esto implica un rango de amplitudes de $10^{9}$.

La banda de frecuencias en la que estamos interesados también es grande, las frecuencias de las ondas sísmicas usadas en sísmica de exploración son tan altas como $1000 \mathrm{~Hz}$, mientras que los mayores períodos de ondas estacionarias excitadas por grandes terremotos tienen frecuencias del orden de $1 \times 10^{-4} \mathrm{~Hz}$ y las frecuencias de las ondas de marea terrestre son del orden de $1.0 \times 10^{-5} \mathrm{~Hz}$. En suma, las movimientos transitorios del suelo cubren un rango de frecuencias de $10^{8} \mathrm{~Hz}$ (Tabla 1.1).

\begin{tabular}{|c||c||}
\hline \multicolumn{1}{|c||}{ Frecuencia (Hz) } & Tipo de mediciones \\
\hline \hline $0.00001-0.0001$ & Mareas Terrestres \\
\hline $0.0001-0.001$ & Oscilaciones libres de la Tierra, Terremotos. \\
\hline $0.001-0.01$ & Ondas superficiales, ondas P y $S$, Terremotos de $\mathrm{M}\rangle 6$ \\
\hline $0.1-10$ & Ondas $\mathrm{P}$ y $\mathrm{S}$, Terremotos con $\mathrm{M}\rangle 2$ \\
\hline $10-1000$ & Ondas $\mathrm{P}$ y $\mathrm{S}$, Terremotos, $\mathrm{M}\langle 2$ \\
\hline
\end{tabular}

Tabla 1.1: Frecuencias típicas generadas por diferentes fuentes sísmicas. Extraído de Havskov y Alguacil, 2002.

De hecho, el estudio de las fuentes sísmicas extiende el rango de interés desde la frecuencia cero o deformación estática, hasta frecuencias del kilohertz que se utilizan en técnicas de estudio de imágenes de alta resolución.

Para un estudio local de corteza se pueden usar ondas que viajan sólo unas decenas de metros, mientras que para el análisis de la estructura global se consideran ondas tales como la $\mathrm{R}_{7}$ (Rayleigh) que viaja más de $10^{8} \mathrm{~m}$ a lo largo de la superficie terrestre, al recorrer 7 veces un círculo máximo. 
Con respecto a la duración de los registros de sismos en los sismogramas, en las fuentes tectónicas las fallas duran segundos a minutos, pero las vibraciones en los sismogramas suelen durar hasta horas. Esto se debe a que la interacción de las ondas con el medio que transmite se manifiesta como una secuencia de arribos impulsivos y movimientos oscilatorios de largos períodos, incluyendo ondas que recorren varias veces el globo, como la ya mencionada.

De acuerdo a lo anteriormente expresado, considerando la gran variedad de señales útiles, uno de los mayores desafíos ha sido construir instrumentos capaces de registrar todo el rango de frecuencias (ancho de banda) y de amplitudes (rango dinámico) teniendo un ambiente ruidoso como trasfondo.

A lo largo de la historia instrumental en Sismología, que data de finales del siglo XIX cuando se comenzaron a construir instrumentos en forma sistemática, no hubo uno único capaz de registrar todo el espectro del movimiento con una respuesta lineal, de modo que fueron desarrollados un conjunto de diferentes instrumentos que registraban porciones limitadas del espectro sísmico y muchas veces trabajaban en conjunto. Sin embargo, en los últimos 25 años se han hecho grandes avances en el desarrollo de instrumental para proveer un amplio ancho de banda y rango dinámico para el estudio de la Sismología global.

La distribución de las fuentes sísmicas, además del requerimiento de una extensa superficie de cobertura con sismómetros para desentrañar e interpretar las complejas señales sísmicas, ha hecho de la Sismología global una verdadera disciplina internacional, con colaboración en relación al desarrollo de los sismómetros e intercambio de datos a través de sus más de 120 años de historia instrumental. Se encuentran en operación alrededor del globo terrestre más de 3000 observatorios sismológicos, con la participación de casi todas las naciones en el esfuerzo de registrar ondas sísmicas continuamente.

\section{2 - Fenómeno físico}

La Sismología involucra el análisis del movimiento del suelo producido por fuentes de energía en la Tierra, tales como terremotos tectónicos o explosiones. Excepto en la inmediata vecindad de la fuente, la mayoría de los movimientos del suelo es efímera; el suelo vuelve a su posición inicial luego que el movimiento transitorio ha terminado. Vibraciones de este tipo involucran deformaciones elásticas pequeñas, como respuesta a fuerzas internas en las rocas o esfuerzos. 
La teoría de la elasticidad provee las relaciones matemáticas entre los esfuerzos y las deformaciones en el medio. El desarrollo de la teoría de la elasticidad que seguiremos está basado en la mecánica de los sólidos, donde un concepto idealizado muy útil para trabajar con fenómenos microscópicos es el de considerar la materia como continuamente distribuida en el espacio, de modo que podemos resolver funciones matemáticas para los desplazamientos, campos de deformación y de esfuerzos con derivadas espaciales bien definidas. La aplicación de la mecánica del continuo permite a los sismólogos explicar casi todas las características de los sismogramas. Debemos luego introducir procesos en escala atómica para explicar algunos aspectos importantes tales como la naturaleza de la atenuación, por ejemplo.

En Sismología global, a grandes distancias se tratan deformaciones muy pequeñas (cambios relativos de longitud de aproximadamente $10^{-6} \mathrm{~m}$ ) sobre períodos cortos de tiempo (<3600 seg.). Esto simplifica el marco matemático de la teoría de elasticidad utilizada, que está basada en la teoría de pequeñas deformaciones. En la cercanía de la fuente o cuando consideramos deformaciones de tiempo finito y de gran escala se debe utilizar una teoría de deformaciones finitas.

La relación entre fuerzas y deformaciones en la teoría de pequeñas deformaciones queda expresada a través de la ley de Hooke. La deformación es una función de las propiedades del material del cuerpo que quedan expresadas a través de su densidad y los módulos elásticos, tales como rigidez (resistencia al corte) e incompresibilidad (resistencia al cambio en volumen).

Cuando el esfuerzo varía con el tiempo, la deformación varía en forma similar y el resultado del balance entre esfuerzo y deformación se traduce en ondas sísmicas. Estas ondas viajan a velocidades que dependen de los módulos elásticos y están gobernadas por ecuaciones de movimiento y se definen como los lugares geométricos determinados por el desplazamiento de las partículas, los que se tornan más complejos a medida que las ondas se expanden a través de los cuerpos sólidos.

Se empleará la descripción Lagrangiana para describir el movimiento de partícula considerándolo como una función del tiempo y el espacio. Esto es natural en Sismología ya que los sismogramas son esencialmente registros de movimientos de partícula, a medida que las ondas pasan, en sensores superficiales o de pozo, ubicados en sitios determinados.

Para llegar a comprender los distintos tipos de ondas que se encuentran en los sismogramas se hará un breve desarrollo de la teoría de elasticidad lineal en un sólido elástico continuo e isótropo al que luego se le aplicarán condiciones de contorno para 
tratar de modelar la Tierra; para tener un análisis detallado se puede consultar Lay and Wallace (1995) y Gershanik (1996), entre otros. En el desarrollo se usará la convención de Einstein: cuando en los factores de un monomio algebraico u operacional aparece un índice repetido, el monomio representa una suma de monomios análogos efectuada sobre todos los valores admisibles de ese índice.

\subsubsection{Ondas de Volumen}

Si se representa con $\vec{\pi}_{v}$ a las tensiones actuantes (fuerzas interiores por unidad de área infinitamente pequeña) en un cuerpo continuo, donde $v$ es la normal exterior a la superficie del lado en que actúan las fuerzas, se demuestra que sus componentes se pueden expresar como:

$$
\left(\overrightarrow{\pi_{v}}\right)_{h}=\tau_{h i} v_{i}
$$

donde $(h=1,2,3)$ y $v_{i}$ y $\tau_{h i}$ son las componentes de la normal $\vec{v}$ y del tensor de las tensiones respectivamente.

Sea $\theta_{1}$ una porción de un cuerpo elástico y continuo $\mathrm{C}$, libre de fuerzas internas. Sean además $\sigma_{1}$ su superficie y $\vec{v}$ la normal exterior de la misma.

Si llamamos a las fuerzas de volumen: $\quad \vec{\pi}^{(\theta)}-\rho \frac{\partial^{2} \vec{s}}{\partial t^{2}}=\vec{P}^{\prime}$

con $\rho$ representando la densidad y estableciendo que $\vec{s}(\vec{x}, t)$ es el vector del campo de movimiento y lo representamos como $\vec{s}=\hat{e}_{h} u_{h}$, entonces la ecuación de equilibrio dinámico para $\theta_{1}$, será la siguiente:

$$
\int_{\theta_{1}} \vec{P}^{\prime} d \theta+\int_{\sigma_{1}} \vec{\pi}_{\nu} d \sigma=0
$$

de donde se puede encontrar que

$$
\left(\vec{P}^{\prime}\right)_{h}+\frac{\partial \tau_{h i}}{\partial \mathbf{x}_{i}}=0
$$

describe la primera condición de equilibrio dinámico en un punto de un cuerpo, que se designa como primera ecuación de equilibrio dinámico local. Esta constituye la ecuación del movimiento, que resulta ser en forma explícita:

$$
\left(\vec{\pi}^{(\theta)}\right)_{h}+\frac{\partial \tau_{h i}}{\partial \mathrm{x}_{i}}=\rho \frac{\partial^{2} u_{h}}{\partial t^{2}} .
$$


Por otra parte, designamos con $\varepsilon_{i j}$ a las componentes del tensor de deformaciones, que quedan definidas por:

$$
\varepsilon_{i j}=\frac{1}{2}\left(\frac{\partial \mathrm{u}_{i}}{\partial \mathrm{x}_{j}}+\frac{\partial \mathrm{u}_{j}}{\partial \mathrm{x}_{i}}\right) \quad(i, j=1,2,3) .
$$

Ya que las componentes del tensor de las tensiones y las componentes del tensor de las deformaciones son consecuencia de una misma causa, cabe considerar a las unas como funciones de las otras. Se puede por ello escribir

$$
\tau_{i j}=f\left(\ldots \varepsilon_{p q} \ldots\right) \text {, }
$$

es decir que una cualquiera de las componentes $\tau_{i j}$ es función de todas las componentes $\varepsilon_{p q}$.

Desarrollando el segundo miembro de (1.6) a partir del estado natural de equilibrio, suponiendo que los $\varepsilon_{p q}$ son muy pequeños lo que en efecto ocurre lejos de las fuentes, y limitando el desarrollo sólo a los términos lineales, resulta para los $\tau_{i j}$ la siguiente expresión:

$$
\tau_{i j}=\mathrm{a}_{i j p q} \varepsilon_{p q}
$$

en la cual los $\mathrm{a}_{i j p q}$ son coeficientes independientes de los $\varepsilon_{p q}$. A dicha expresión se la conoce con el nombre de Ley de Hooke generalizada. En general, los $\mathrm{a}_{i j p q}$ pueden variar con las coordenadas del punto del cuerpo que se considere. Si ello no ocurre se dice que el cuerpo es elásticamente homogéneo.

Como en esa expresión los $\tau_{i j}$ aparecen vinculados linealmente con los $\varepsilon_{p q}$ se dice que la teoría que sobre ella se funda es una teoría de la Elasticidad lineal, y en virtud de que en ella se supone que los $\varepsilon_{i j}$ son pequeños, también que es una teoría de deformaciones infinitesimales. Esta expresión implica entonces un medio lineal y es aplicable lejos de las fuentes. A los coeficientes $\mathrm{a}_{i j p q}$ se los conoce con el nombre de parámetros elásticos.

Se demuestra que las componentes del tensor de tensiones en un medio elástico, lineal e isótropo estarán dadas por

$$
\tau_{h i}=\lambda \Theta \delta_{h i}+2 \mu \varepsilon_{h i},
$$

siendo $\lambda$ y $\mu$ los coeficientes de Lamé y $\Theta$ la divergencia del campo de desplazamientos, con $\Theta=\varepsilon_{11}+\varepsilon_{22}+\varepsilon_{33}$. 
Esta expresión fue formulada por Navier en 1821 y Cauchy en 1823, 160 años después de la ley de Hooke.

Si se introduce la expresión (1.8) en la primera ecuación de equilibrio local (1.4), dicha ecuación se transforma en la siguiente:

$$
\begin{aligned}
& \vec{P}_{h}^{\prime}+\lambda \frac{\partial \Theta}{\partial \mathrm{x}_{i}} \delta_{h i}+2 \mu \frac{\partial \varepsilon_{h i}}{\partial \mathrm{x}_{i}}=0 \quad \text { de donde recordando (1.5), resulta } \\
& \left(\vec{\pi}^{(\theta)}\right)_{h}+(\lambda+\mu) \frac{\partial \Theta}{\partial \mathrm{x}_{h}}+\mu \frac{\partial}{\partial \mathrm{x}_{i}}\left(\frac{\partial \mathrm{u}_{h}}{\partial \mathrm{x}_{i}}\right)-\rho \frac{\partial^{2} \mathrm{u}_{h}}{\partial \mathrm{t}^{2}}=0 .
\end{aligned}
$$

Como estas ecuaciones valen para todos los valores de $h$, se las puede presentar en forma vectorial como sigue:

$$
\hat{\mathrm{e}}_{h}\left(\vec{\pi}^{(\theta)}\right)_{h}+(\lambda+\mu) \frac{\partial \Theta}{\partial \mathrm{x}_{h}} \hat{\mathrm{e}}_{h}+\mu \frac{\partial}{\partial \mathrm{x}_{i}}\left(\frac{\partial \mathrm{u}_{h}}{\partial \mathrm{x}_{i}}\right) \hat{\mathrm{e}}_{h}-\rho \frac{\partial^{2} \mathrm{u}_{h}}{\partial \mathrm{t}^{2}} \hat{\mathrm{e}}_{h}=0
$$

A las ecuaciones (1.10) y (1.11) se las conoce con el nombre de ecuaciones del movimiento del cuerpo isótropo.

Debido a que

$$
\frac{\partial}{\partial \mathrm{x}_{i}}\left(\frac{\partial \mathrm{u}_{h}}{\partial \mathrm{x}_{i}}\right)=\nabla^{2} \mathrm{u}_{h}
$$

y

$$
\Theta=\operatorname{div} \overrightarrow{\mathrm{s}}
$$

puede presentarse la (1.11) también como

$$
(\lambda+\mu) \operatorname{grad} \operatorname{div} \overrightarrow{\mathrm{s}}+\mu \nabla^{2} \overrightarrow{\mathrm{s}}+\overrightarrow{\mathrm{P}}-\rho \frac{\partial^{2} \overrightarrow{\mathrm{s}}}{\partial \mathrm{t}^{2}}=0,
$$

entendiéndose que la operación $\nabla^{2}$, conocida como Laplaciana, debe aplicarse a las componentes de $\vec{s}$ y que $\vec{P}$ representa las fuerzas de volumen $\hat{e}_{h}\left(\vec{\pi}^{(\theta)}\right)_{h}$.

Para encontrar la solución de esta ecuación se puede aplicar el método de los potenciales utilizando la descomposición de Helmholtz.

Se podrá poner para el vector desplazamiento:

$$
\overrightarrow{\mathrm{s}}=\operatorname{grad} \varphi+\operatorname{rot} \vec{\Psi}
$$

que como se ve, expresa que el vector $\overrightarrow{\mathrm{s}}$ puede considerarse formado por el gradiente de un campo potencial escalar $\varphi$ irrotacional $(\operatorname{rot} \varphi=0)$ y por el campo de un potencial vectorial $\Psi$ no divergente $(\operatorname{div} \Psi=0)$. Físicamente un campo irrotacional involucra que no hay movimiento de corte y un campo no divergente que no hay cambios de volumen. 
Por sencillez para encontrar la solución supongamos que no existan fuerzas de volumen en (1.12). Con ello esa ecuación se reduce a la siguiente:

$$
(\lambda+\mu) \operatorname{grad} \operatorname{div} \overrightarrow{\mathrm{s}}+\mu \nabla^{2} \overrightarrow{\mathrm{s}}-\rho \frac{\partial^{2} \overrightarrow{\mathrm{s}}}{\partial \mathrm{t}^{2}}=0 .
$$

Si se reemplazara $\overrightarrow{\mathrm{s}}$ por su representación dada por (1.13), entonces se satisfacen las siguientes ecuaciones:

$$
\begin{gathered}
\frac{\lambda+2 \mu}{\rho} \nabla^{2} \varphi-\frac{\partial^{2} \varphi}{\partial \mathrm{t}^{2}}=0 \\
\mathrm{y} \quad\left[\frac{\mu}{\rho} \nabla^{2} \mathrm{e}_{i j k} \frac{\partial \phi_{j}}{\partial \mathrm{x}_{i}}-\frac{\partial^{2}}{\partial \mathrm{t}^{2}} \mathrm{e}_{i j k} \frac{\partial \phi_{j}}{\partial \mathrm{x}_{i}}\right] \hat{\mathrm{e}}_{k}=0,
\end{gathered}
$$

donde $e_{i j k}$ es el tensor de permutaciones.

De ellas, la (1.15) es una ecuación escalar y la (1.16) es una ecuación vectorial. Como la validez de esta última exige la nulidad de cada una de las componentes, la misma representa en rigor tres ecuaciones, por lo que en total en (1.15) y en (1.16) tenemos cuatro ecuaciones que claramente son ecuaciones de onda que se propagan con velocidades:

$$
\sqrt{\frac{\lambda+2 \mu}{\rho}}=\alpha \quad \text { y } \quad \sqrt{\frac{\mu}{\rho}}=\beta
$$

respectivamente. En todos los casos el campo de desplazamientos comprende dos tipos fundamentales de ondas, que se propagan con distintas velocidades determinadas por las propiedades de los materiales del medio. Las primeras reflejan movimientos compresionales y cambios de volumen a medida que la perturbación se propaga, mientras que las segundas representan movimientos de cizalla, sin cambios de volumen.

En Sismología se identifica $\alpha$, velocidad de la solución de la onda $\varphi$, como velocidad de las ondas $\mathrm{P}$ y $\beta$ como velocidad de las ondas $\mathrm{S}$, que corresponde a las soluciones $\Psi$. Encontrando las soluciones a los potenciales, a través de (1.13) se puede determinar el campo de desplazamientos. De las expresiones de las velocidades es claro que $\alpha\rangle \beta$, de modo que las ondas $\mathrm{P}$ arriban antes que las ondas $\mathrm{S}$ a las estaciones sismológicas. Se puede demostrar que estas ondas son las únicas soluciones transitorias para un medio totalmente elástico y homogéneo; de modo que 
juntas proveen la solución completa para la ecuación de movimiento (ver Lay and Wallace, 1995).

Para encontrar la solución de la (1.15) en coordenadas cartesianas $x_{i}$ se puede aplicar el método de separación de variables, con el que se llega a que una solución particular será:

$$
\varphi\left(x_{1}, x_{2}, x_{3}, t\right)=A\left(k_{1}, k_{2}, k_{3}, \omega\right) e^{ \pm i\left(\omega t-k_{l} x_{l}\right)}
$$

función del tipo D' Alembert que depende del espacio y del tiempo siendo el argumento de la exponencial, la fase.

Esta solución corresponde a un conjunto de ondas planas, libres de propagarse en cualquier dirección del medio. El requerimiento para una dada frecuencia angular $\omega$ y velocidad $\alpha$ que $k_{1}^{2}+k_{2}^{2}+k_{3}^{2}=$ cte, define una superficie plana en el espacio cartesiano, con un vector normal $\overrightarrow{k_{\alpha}}=\frac{\omega}{\alpha} \hat{k}$ llamado vector número de onda que determina la dirección de propagación de la onda (la normal al plano de onda). Podemos escribir la solución de la forma:

$$
\varphi(\vec{x}, t)=A e^{i\left(\omega t-\vec{k}_{\alpha} \cdot \vec{x}\right)}
$$

y en forma análoga:

$$
\Psi(\vec{x}, t)=B e^{i\left(\omega t-\overrightarrow{k_{\beta}} \cdot \vec{x}\right)}
$$

en las cuales, definiendo $T$ como el periodo, $\lambda$ la longitud de onda, $\omega$ la frecuencia angular y $f$ la frecuencia, se verifica que:

$$
\omega=2 \pi f, \omega=\frac{2 \pi}{T}, \alpha=\frac{\lambda}{T} \Rightarrow\left|k_{\alpha}\right|=\frac{2 \pi}{\lambda},
$$

con $A$ y $B$ constantes arbitrarias, llamadas las amplitudes de onda.

$\mathrm{Si}$ en el material hay presentes inhomogeneidades localizadas $\mathrm{O}$ discontinuidades, los campos de ondas analizados se tornan más complicados. Un medio inhomogéneo tal como la Tierra, donde ocurren fenómenos de onda tales como refracción, conversión, dispersión dependiente de la frecuencia y difracción, hace que el campo de ondas de las ondas corpóreas se vuelva más complejo.

El hecho que las inhomogeneidades de la Tierra, en una primera aproximación se puedan considerar en una dimensión (radial), nos permite interpretar en gran medida la complejidad de las ondas corpóreas. 
La Tierra tiene atributos adicionales fundamentales que afectan profundamente a los campos de ondas corpóreas, el primero de ellos compartido con todas las estructuras finitas: la presencia de una superficie libre, un interior con discontinuidades que separa medios con parámetros elásticos distintos, y su forma casi elipsoidal.

La Tierra finita, así como su constitución interna en capas, provee entonces longitudes de escala y condiciones de borde para las ondas sísmicas. Podemos tratar el planeta como un sistema elástico finito con condiciones de borde únicas que gobiernan las soluciones de las ecuaciones de movimiento en el medio. Esta perspectiva nos lleva a la definición de modos normales del sistema, que involucran frecuencias discretas a las cuales el sistema puede oscilar, de una manera análoga a los tonos armónicos de un órgano o a las cuerdas de una guitarra.

\subsubsection{Ondas Superficiales.}

La superficie libre de un medio elástico determina un comportamiento especial del esfuerzo, ya que las tensiones se desvanecen en la superficie. Como las mediciones de ondas sísmicas se realizan en o cerca de la superficie es importante comprender los efectos de esta superficie libre para poder comprender los sismogramas. En la superficie coexisten instantáneamente las ondas incidentes y reflejadas, y el movimiento total involucra la suma de sus respectivas amplitudes.

La interacción de ondas incidentes $\mathrm{P}$ y SV (componente $\mathrm{S}$ vertical) con la condición de borde de superficie libre, da lugar a una onda que viaja efectivamente a través de la superficie llamada onda Rayleigh. La reflexión total de ondas SH (componente $S$ horizontal) en la superficie libre combinada con las capas internas de la Tierra para constituir ondas SH estacionarias, produce una propagación horizontal llamada ondas Love.

En efecto, si se considera que ondas P y SV se propagan simultáneamente a lo largo de la superficie libre y se asume que los potenciales tienen la forma:

$$
\begin{aligned}
& \phi=A \exp \left[i \omega\left(p x_{1}+\eta_{\alpha} x_{3}-t\right)\right] \\
& \psi=B \exp \left[i \omega\left(p x_{1}+\eta_{\beta} x_{3}-t\right)\right]
\end{aligned}
$$

donde $\mathrm{c}$ es la velocidad horizontal aparente, $c=(1 / p), c\langle\beta\langle\alpha$ con 


$$
\eta_{\alpha}=\sqrt{\frac{1}{\alpha^{2}}-p^{2}}=i \sqrt{\frac{1}{c^{2}}-\frac{1}{\alpha^{2}}}
$$

y

$$
\eta_{\beta}=\sqrt{\frac{1}{\beta^{2}}-p^{2}}=i \sqrt{\frac{1}{c^{2}}-\frac{1}{\beta^{2}}}
$$

obligando a que la energía se propague a lo largo de la superficie con decaimiento exponencial de los potenciales al apartarse de la superficie $x_{3}=0$, se encuentra que esta condición de borde se satisface para una onda acoplada P-SV viajando a una velocidad menor que la velocidad de las ondas de corte. Esto fue hallado por Lord Rayleigh en 1887.

Las ondas Rayleigh resultantes tienden a ser los arribos de mayor amplitud en sismogramas de banda ancha o largo período, lo cual resulta de la expansión geométrica en dos dimensiones de la onda superficial con respecto a los efectos en tres dimensiones que afectan a las ondas corpóreas.

Para una estructura inhomogénea, las ondas Rayleigh son dispersivas, dando origen a mayores velocidades para longitudes de onda grandes y componentes de baja frecuencia. Las fuentes cercanas a la superficie tienden a excitar ondas Rayleigh fuertes, mientras que fuentes profundas excitan sólo ondas Rayleigh débiles.

La componente $\mathrm{SH}$ de la onda $\mathrm{S}$, que tiene desplazamientos paralelos a la superficie, puede solamente tener reflexiones totales desde la superficie libre. Para atrapar alguna energía SH cerca de la superficie, la estructura de velocidades con la profundidad debe ser tal que mantenga energía en la superficie. Si la velocidad de las ondas $S$ aumenta con la profundidad, se puede formar una onda estacionaria, en la cual los rayos desarrollan reflexiones múltiples entre la superficie y los puntos de reflexión. Cuando los rayos arriban al horizonte de reflexión con ángulos mayores que el crítico, toda la energía queda atrapada en la onda estacionaria. Estas ondas llevan el nombre de ondas Love.

Estas ondas quedan representadas a través de los desplazamientos $\mathrm{SH}$, que satisfacen la ecuación de onda:

$$
\begin{gathered}
V_{1}=A \exp \left[i \omega\left(p x_{1}+\eta_{\beta_{1}} x_{3}-t\right)\right]+B \exp \left[i \omega\left(p-\eta_{\beta_{1}} x_{3}-t\right)\right] \\
V_{2}=C \exp \left[i \omega\left(p x_{1}+\eta_{\beta_{2}} x_{3}-t\right)\right]
\end{gathered}
$$


donde $V_{1}$ es el desplazamiento en la capa, que involucra ondas planas ascendentes y descendentes, y $V_{2}$ es el desplazamiento $\mathrm{SH}$ en el semiespacio, compuesto por ondas $\mathrm{SH}$ transmitidas generadas en cada punto de reflexión en la base de la capa.

Recordando la ley de Snell, si $\left.\beta_{1}\right\rangle \beta_{2}$, entonces $j_{3}\left\langle j_{1}\right.$ (donde $j_{3}$ es el ángulo de refracción en el semiespacio y $j_{1}$ el ángulo de incidencia en la capa) y la energía transmitida se propagará alejándose de la capa de mayor velocidad, con reverberaciones en la misma disminuyendo progresivamente. Para $\beta_{1}\left\langle\beta_{2}, j_{3}\right\rangle j_{1}=j_{2}$, la onda transmitida es refractada más cerca al límite pero aún se propaga alejándose, perdiendo energía en la capa de baja velocidad, hasta que $j_{1}=j_{c}=\operatorname{sen}^{-1}\left(\beta_{1} / \beta_{2}\right)$, el ángulo crítico, en el cual la onda transmitida se refracta a lo largo del borde, "head wave". Para ángulos iguales o mayores que el crítico, el coeficiente de reflexión de la onda transversal $B / A$ vale 1 y adquiere un atraso de fase. La energía de la onda SH es entonces totalmente reflejada tanto en el borde como en la superficie libre y la onda SH postcrítica quedará "atrapada" en la capa.

Las ondas Love son siempre dispersivas ya que requieren para existir al menos una capa de baja velocidad por encima de un espacio semi-infinito. Hay una completa separación entre el movimiento superficial de ondas Love y Rayleigh, ya que las ondas Love se propagan más rápido y arriban en la componente transversal antes que la Rayleigh, que lo hace en las componentes vertical y radial.

La geometría particular de la Tierra tiene un importante efecto en la propagación de las ondas superficiales. Las ondas superficiales convergen desde todas las direcciones hacia la antípoda del epicentro; las ondas Rayleigh con una interferencia constructiva que determina amplitudes verticales grandes, mientras que las ondas Love no provocan movimientos ya que interfieren destructivamente.

\section{3 - Sismogramas}

En un modelo de Tierra ideal que asume un medio lineal, homogéneo e isótropo, aparecen un conjunto de ondas que bajo estas condiciones no están acopladas: P, S, Love y Rayleigh, cada una con distintas velocidades, con lo cual el registro de un sismo se vería ya complejo.

En la Tierra real, cuyos parámetros elásticos sufren variaciones y además posee discontinuidades, estos fenómenos descriptos sufren alteraciones ya que son 
sometidos a efectos de reflexión, refracción, dispersión, atenuación y variaciones laterales, lo que produce sismogramas sumamente complejos (Fig. 1.1).

Es claro entonces que un simple modelo de capas para la corteza terrestre también nos permite una aproximación pobre. La diferencia obvia entre las regiones continentales y oceánicas son una indicación de esto, además la geología superficial provee evidencia clara de la complejidad. Los procesos geológicos que producen capas, tales como la deposición sedimentaria, flujos de lava y precipitación química, ocurren en escalas espaciales limitadas y los subsecuentes movimientos corticales

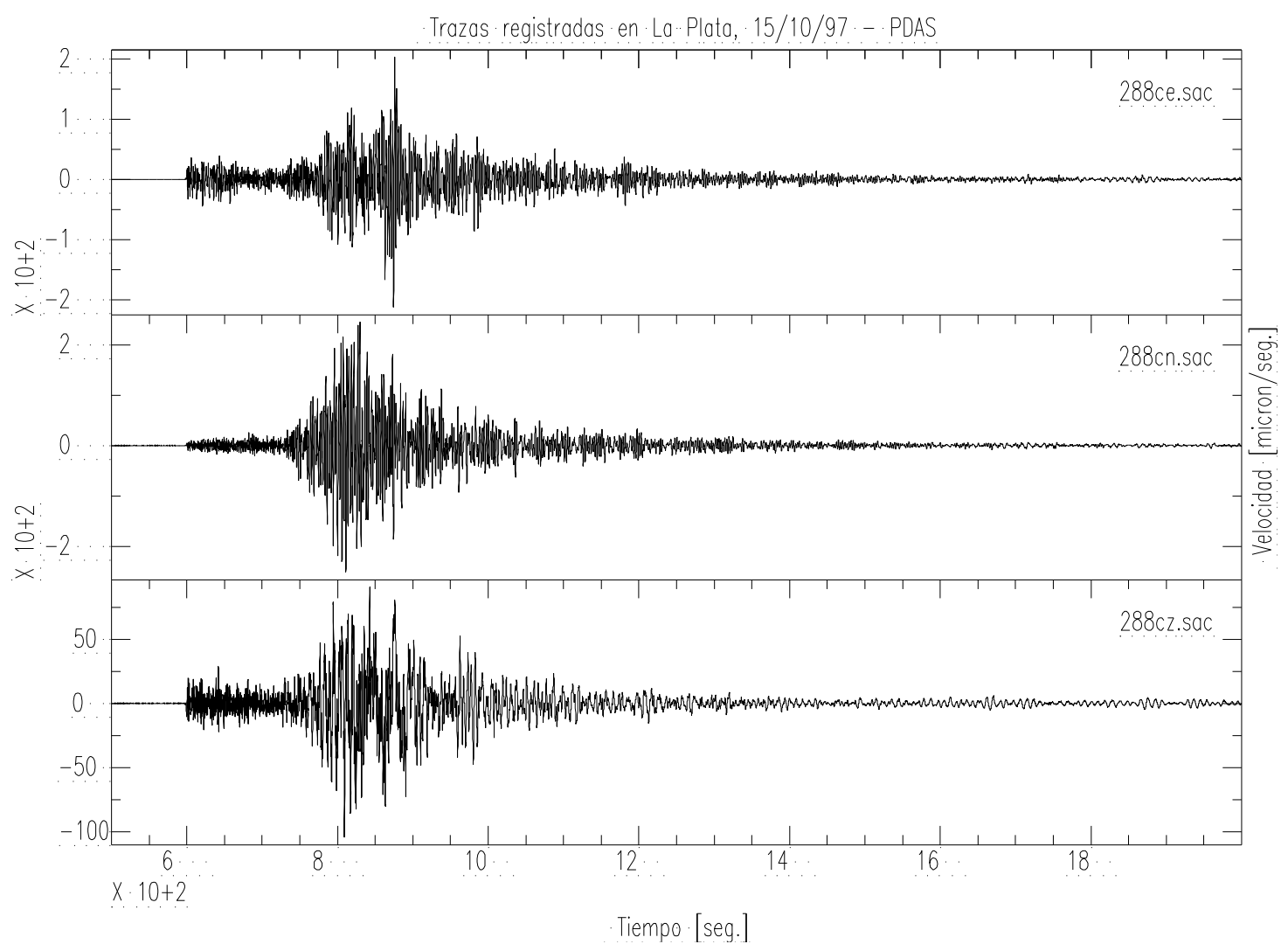

Figura 1.1: Ejemplo de sismograma obtenido en la estación sismológica de La Plata, con sensores BB13 y adquisidor PDAS100. Terremoto ocurrido en Chile: 30.933S, 71.220W, Prof. 58km, Mag. 7.1.

deforman hasta las rocas estratificadas localmente, lo que se traduce obviamente en la existencia de variaciones laterales que quedan reflejadas o representadas en los sismogramas.

En la década del 80 los sismólogos globales iniciaron un análisis exhaustivo de diferentes tipos de datos sísmicos para determinar la estructura de la Tierra en tres dimensiones usando métodos llamados colectivamente como tomografía sísmica, 
encontrando que cada región del interior del planeta, con la posible excepción del núcleo exterior, tiene heterogeneidades no esféricas detectables. Además teniendo en cuenta que las variaciones de velocidad se pueden asociar a variaciones térmicas, (velocidades mayores para materiales más fríos y velocidades menores para materiales más calientes a igual presión), y que estas variaciones implican cambios de densidad, los modelos sísmicos en tres dimensiones revelan heterogeneidades en la densidad que se traducen en esfuerzos en largos períodos de tiempo en los que los materiales terrestres fluyen transportando calor a la superficie.

\section{4 - El ruido en la Tierra}

Los sismógrafos sensibles registrarán movimientos del suelo incluso en ausencia de terremotos. Estos movimientos, algunas veces llamados microsismos, resultan de las ondas sísmicas generadas principalmente por el viento y el ruido cultural en altas frecuencias $u$ ondas oceánicas y efectos atmosféricos en largos períodos. Aunque los microsismos son estudiados a veces por su interés intrínseco, los sismólogos generalmente los consideran ruido porque ellos enmascaran las señales de terremotos pequeños y/o distantes. Se puede hacer una descripción detallada de los microsismos en función de su origen:

Ruido antropogénico: a menudo se lo menciona como ruido "cultural", se origina por el tráfico y por la industria, tiene altas frecuencias $(>2-4 \mathrm{~Hz})$ y decrece rápidamente cuando uno se aleja de la fuente del ruido. Se propaga principalmente en altas frecuencias en ondas superficiales, que se atenúan rápidamente con la distancia y decrecen en amplitud fuertemente con la profundidad, de modo que se tornan prácticamente imperceptibles en pozos, cavernas profundas y túneles. Esta clase de ruido usualmente presenta una gran diferencia entre el día y la noche y puede tener frecuencias características debido a la fuente de perturbación presente. El nivel de este ruido puede ser muy alto.

Ruido del viento: el viento hace que todo objeto se mueva de modo que siempre genera perturbación en el suelo. Este ruido es usualmente de alta frecuencia como el ruido cultural; sin embargo, objetos grandes ondulando como mástiles y torres pueden generar señales de baja frecuencia. Los árboles también transmiten las vibraciones del viento al suelo y en consecuencia las estaciones sismológicas deberían estar instaladas lejos de ellos. En general, las turbulencias del viento sobre las topografías 
irregulares como escarpas o rocas generan ruido local y su proximidad debería también ser evitada.

Ruido generado por el océano: este es el ruido que más se ve globalmente, aunque en el interior de los continentes es menor que en las regiones costeras. En aguas someras en regiones costeras se generan sólo microsismos oceánicos de largo período, donde la energía de las ondas se convierte directamente en energía sísmica a través de variaciones verticales de presión. Entonces tienen el mismo período que las ondas oceánicas de 10 a 16 seg. Hay microsismos de períodos menores cuyo mecanismo físico de generación fue un misterio durante muchos años. Este pico microsísmico principal fue explicado por primera vez por Longuet-Higgins (1950), quien mostró que resulta de la superposición de ondas oceánicas del mismo período propagándose en direcciones opuestas, que generan ondas estacionarias de gravedad de la mitad del período. Estas ondas estacionarias causan perturbaciones que se propagan sin atenuación al fondo del océano. Los microsismos de mayores frecuencias tienen amplitudes mayores que los microsismos de baja frecuencia. Estos microsismos se forman a menudo en áreas de grandes tormentas, en las que la amplitud puede llegar a ser de $20000 \mathrm{~nm}$ en estaciones cercanas a la costa y pueden hacer que algunos sismogramas analógicos se tornen inutilizables.

Otras fuentes: agua en movimiento, tremors volcánicos y de superficie (en casi todos los ruidos armónicos asociados con fluidos en movimiento, a menudo durando horas o días) o actividad de bajo nivel son otras fuentes de ruido sísmico.

El ruido cultural y el ruido del viento son usualmente las principales fuentes de frecuencias altas y ya que el nivel de señal menor es aproximadamente $0.01 \mathrm{~nm}$ a 10 $\mathrm{Hz}$, pequeñas perturbaciones llevarán rápidamente el nivel de ruido sobre este valor.

Los niveles típicos de ruido pueden variar mucho entre diferentes sitios y diferentes frecuencias. Esto se ilustra en la Fig. 1.2, donde se muestran niveles típicos de ruido alrededor de 100 estaciones diferentes de una red sísmica global (Astiz, 1997).

Se ve un gran pico en el ruido que ocurre para períodos entre 5 a 8 seg; esto es llamado pico del microsismo y la fuente de este pico involucra las ondas sísmicas generadas en los océanos; el pico es observado más fuertemente a lo largo de la costa y es más débil cerca del centro de los continentes; son las ondas estacionarias explicadas anteriormente.

Durante la década del 40 se propuso que se podían usar pequeños arreglos de sismómetros para determinar las ubicaciones de las fuentes de los microsismos y 
potencialmente determinar los recorridos de los huracanes antes que llegaran a tierra. Este esquema no resultó muy bueno, y, en cualquier caso, hoy en día es obsoleto

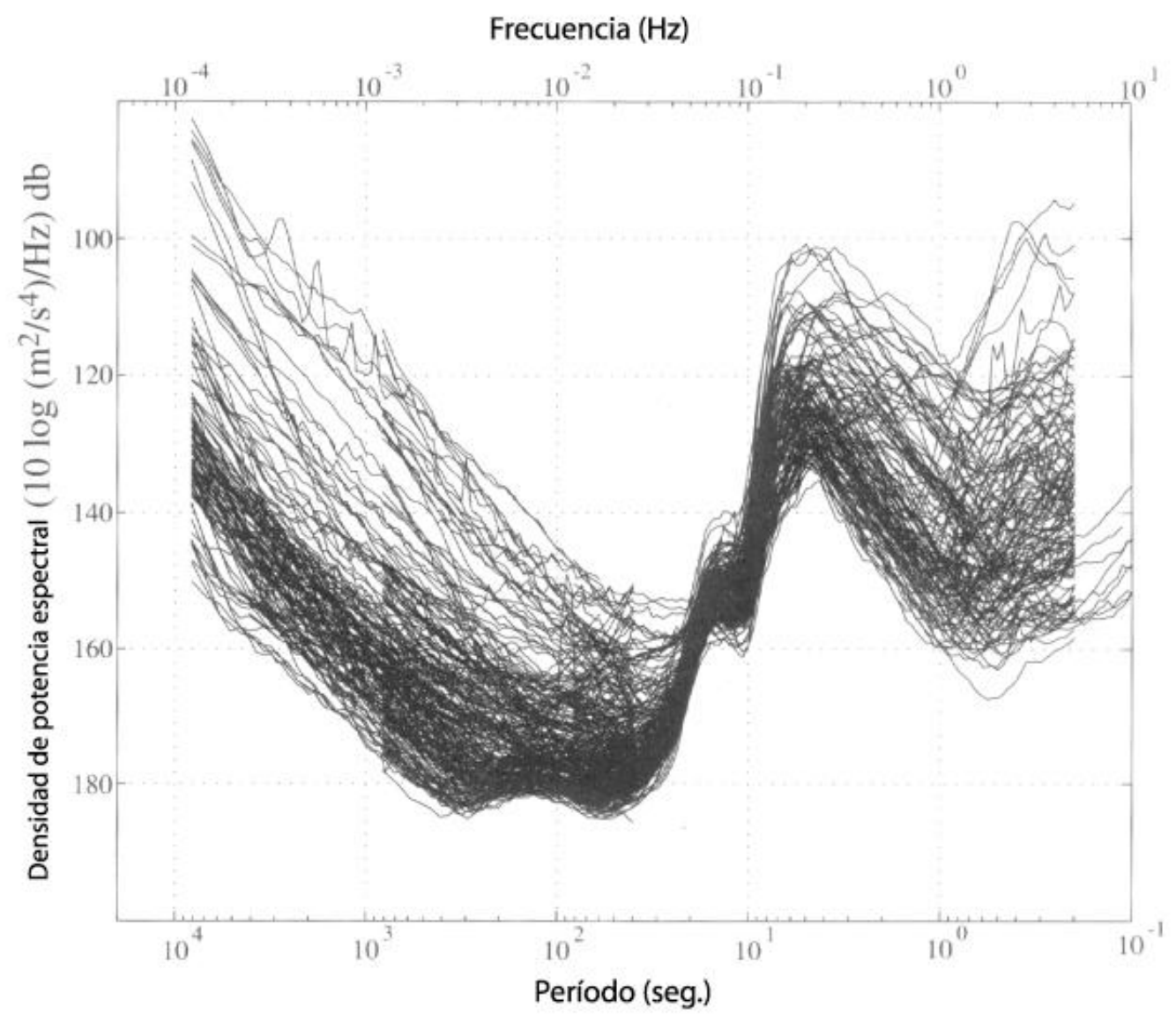

Figura 1.2: Ruido de la Tierra expresado a través de la densidad de potencia espectral, de Astiz, 1997.

debido a los observaciones satelitales. Los microsismos son ahora estudiados mucho menos de lo que lo eran cincuenta años atrás, aunque recientemente ha habido un incremento en su utilización para caracterizar velocidades cercanas a la superficie y la respuesta de sitio de estaciones sismológicas.

El interés por los microsismos también involucra el estudio de cómo reducir su influencia en las observaciones sísmicas. Los niveles de ruido en las instalaciones de sismómetros varían mucho entre distintos sitios; un sitio quieto es capaz de detectar y registrar muchos más terremotos que un sitio ruidoso. Los niveles de ruido en los continentes son generalmente más bajos a grandes distancias de la costa, mientras que los sitios más ruidosos están ubicados en el fondo del océano y en las islas oceánicas. Algunas mejoras en los niveles de ruido pueden obtenerse con pozos o instalaciones en minas, debajo del nivel de la superficie. 
Para frecuencias extremadamente bajas, las mayores señales son las mareas sólidas de la Tierra, que ocurren en períodos de 12 y 24 horas y son registradas solamente con sensores que sean estables en estos períodos. Las mareas terrestres pueden ser usadas para ayudar en la calibración de sismógrafos de muy largo período y sismómetros de deformación, ya que pueden ser predichas con modelos estándar de Tierra, aunque se debe tener en cuenta de incluir las mareas oceánicas cerca de las líneas costeras. 


\section{Capítulo 2 \\ ESTACIÓN SISMOLÓGICA MODERNA}

\section{1 - Instrumentación}

El objetivo es medir el movimiento de la Tierra en un punto con respecto a ese mismo punto no perturbado. Desafortunadamente no es tan fácil medir este movimiento y el sensor sísmico es el elemento unitario más crítico de un sismógrafo (sismómetro y unidad de registración). Las principales dificultades son:

1.- La medición es hecha en un marco de referencia móvil, en otras palabras, el sensor se está moviendo con el suelo y no hay ninguna referencia fija disponible, de modo que el desplazamiento no puede ser medido directamente. De acuerdo al principio de inercia, sólo se puede observar este movimiento si tiene alguna aceleración. Las ondas sísmicas ocasionan movimientos transitorios y esto implica que debe haber aceleración. La velocidad y el desplazamiento pueden ser estimados, pero estos sismómetros, llamados inerciales no pueden detectar cualquier componente continua de ellos.

2.- El rango de amplitudes y frecuencias de las señales sísmicas es muy grande, como ya se ha mencionado y probablemente nunca será posible hacer un sensor que los pueda cubrir en su totalidad. No obstante ello, una buena estación sismológica para estudios locales y globales debería al menos cubrir la banda de frecuencias de 0.01 a $100 \mathrm{~Hz}$ y movimientos de la Tierra desde $1 \mathrm{~nm}$ hasta $10 \mathrm{~mm}$.

Ya que no es posible hacer un único instrumento que cubra este rango de valores, se utilizan instrumentos con diferentes ganancias y respuesta en frecuencia para diferentes rangos de frecuencia y amplitud.

El sismómetro (sensor) que detecta el movimiento del suelo $\vec{u}(\vec{x}, t)$ más común está basado en la inercia de una masa suspendida, que tenderá a permanecer en reposo frente a vibraciones externas.

Como ejemplo, se puede mostrar un sismómetro de diseño simple que detecta movimientos verticales del suelo (Fig. 2.1). Una masa está suspendida de un resorte y conectada a una barra de manera que sólo se puede mover en la dirección vertical.

El movimiento relativo entre la masa y el soporte del sismómetro se puede medir a través del voltaje inducido en una bobina por el movimiento de un imán. El voltaje inducido es proporcional a la velocidad de la masa para el instrumento mostrado en la figura. En otros diseños de sensores, pueden ser medidos el 
desplazamiento o la aceleración de la masa. La respuesta en frecuencia del sismómetro es función de la variable que se mida. Se puede realizar un diseño similar para detectar el movimiento horizontal del suelo; en este caso la masa está suspendida como un péndulo.

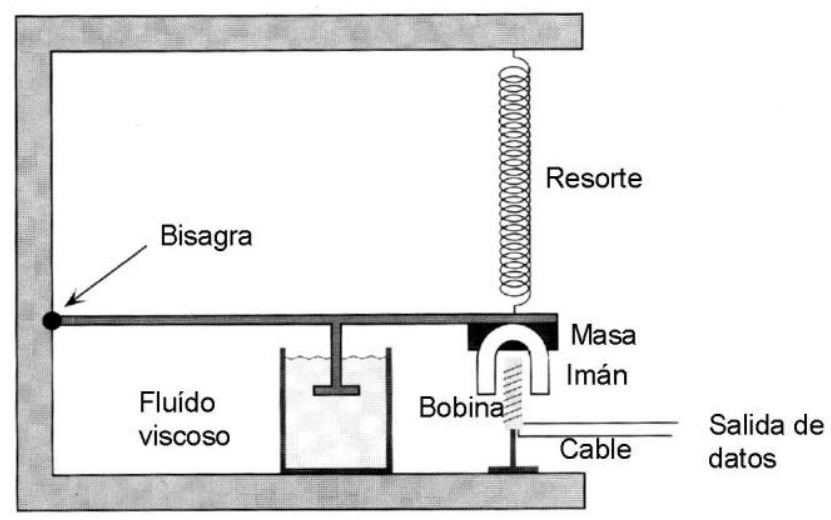

Figura 2.1. Componente vertical de sismómetro inercial elemental, modificado de Shearer 1999.

El movimiento de la Tierra se relaciona con el movimiento de la masa suspendida a través de la función de respuesta que incluye los efectos de las fuerzas que actúan sobre la masa. Esta función se puede derivar fácilmente para sismómetros inerciales simples como el mostrado en el gráfico (ver por ejemplo, Gershanik, 1996).

Sea $\vec{u}(t)$ el desplazamiento vertical de la Tierra y $\vec{z}(t)$ el desplazamiento de la masa con respecto a la Tierra, ambos relativos a su posición de reposo. Asumimos que el soporte del sismómetro está rígidamente conectado a la Tierra, de modo tal que $\vec{z}(t)$ representa el desplazamiento con respecto al soporte. El desplazamiento absoluto de la masa está dado por la suma $\vec{u}(t)+\vec{z}(t)$. La fuerza que ejerce el resorte sobre la masa, $\vec{F}_{s}$, se opondrá al desplazamiento de la masa y está dado por:

$$
\vec{F}_{s}=-k \vec{z}
$$

donde $k$ es la constante del resorte. La fuerza de amortiguamiento viscoso, $\vec{F}_{d}$, es proporcional a la velocidad de la masa y está dada por:

$$
\vec{F}_{d}=-D \frac{d \vec{z}}{d t}
$$

siendo $D$ la constante de amortiguamiento. Se tiene entonces, llamando $\mathrm{m}$ a la masa: 


$$
-k \vec{z}(t)-D \frac{d \vec{z}(t)}{d t}=m \frac{d^{2}}{d t^{2}}[\vec{u}(t)+\vec{z}(t)]
$$

que se puede escribir como:

$$
\overrightarrow{\ddot{z}}(t)+\frac{D}{m} \overrightarrow{\dot{z}}(t)+\frac{k}{m} \vec{z}(t)=-\overrightarrow{\ddot{u}}(t)
$$

Si se define $\omega_{0}^{2}=\frac{k}{m}$, donde $\omega_{0}$ es la frecuencia angular de resonancia para el sistema no amortiguado $(D=0)$ y $\varepsilon$ el parámetro de amortiguamiento, tal que $2 \varepsilon=\frac{D}{m}$, se obtiene:

$$
\overrightarrow{\ddot{z}}(t)+2 \overrightarrow{\dot{z}}(t) \varepsilon+\omega_{0}^{2} \vec{z}(t)=-\overrightarrow{\ddot{u}}(t)
$$

Esta ecuación muestra que la aceleración de la Tierra, $\overrightarrow{\ddot{u}}(t)$, puede ser recuperada midiendo el desplazamiento de la masa, $\vec{z}(t)$, y sus derivadas.

La función de transferencia del sismómetro puede ser también expresada en el dominio de las frecuencias. Si se considera que la Tierra tiene un desplazamiento armónico de la forma:

$$
\vec{u}(t)=U(\omega) e^{-i \omega t}
$$

donde $\omega=2 \pi f$ es la frecuencia angular, la respuesta en desplazamiento de la masa del sismómetro puede ser expresada como

$$
\vec{z}(t)=Z(\omega) e^{-i \omega t}
$$

Entonces se tiene:

$$
\begin{aligned}
& \overrightarrow{\ddot{u}}(t)=-\omega^{2} U(\omega) e^{-i \omega t} \\
& \overrightarrow{\dot{z}}(t)=-i \omega Z(\omega) e^{-i \omega t} \\
& \vec{z}(t)=-\omega^{2} Z(\omega) e^{-i \omega t}
\end{aligned}
$$

Sustituyendo y dividiendo por el factor común $e^{-i \omega t}$, se obtiene:

$$
-\omega^{2} Z(\omega)-2 \varepsilon i \omega Z(\omega)+\omega_{0}^{2} Z(\omega)=\omega^{2} U(\omega)
$$

0 :

$$
Z(\omega)=\frac{\omega^{2}}{\omega_{0}^{2}-2 i \varepsilon \omega-\omega^{2}} U \omega=\mathrm{T}(\omega) U(\omega)
$$


donde $\mathrm{T}(\omega)$ es la función de transferencia en frecuencia del sensor $(\mathrm{T}(\omega)=Z(\omega)$ cuando $U(\omega)=1)$. La función de transferencia $\mathrm{T}(\omega)$ es compleja y puede ser expresada como:

$$
\mathrm{T}(\omega)=A(\omega) e^{i \phi(\omega)}
$$

donde la amplitud $A(\omega)=|\mathrm{T}(\omega)|$, y la fase $\phi$ son números reales.

La función de transferencia también puede representarse utilizando la transformación integral de Laplace ya que toda señal $f(t)$ que tenga un comienzo definido en el tiempo puede ser descompuesta en señales sinusoidales exponencialmente crecientes $(\sigma>0)$ o decrecientes $(\sigma<0)$ :

$$
f(t)=\frac{1}{2 \pi j} \int_{\sigma-j \infty}^{\sigma+j \infty} F(s) e^{s t} d s \quad F(s)=\int_{0}^{\infty} f(t) e^{-s t} d t
$$

La primera integral define la transformación inversa y la segunda la transformación directa, $s$ es una variable compleja que puede tomar cualquier valor para el cual la segunda integral converja. La transformada de Laplace $F(s)$ se dice entonces que "existe" para este valor de $s$. Si llamamos $G(s)$ a la transformada de Laplace de la señal de salida, generalmente para todo sistema lineal e invariante temporalmente:

$$
G(s)=H(s) \cdot F(s)
$$

en la que la función $H(s)$ es la función de transferencia del sistema que desde el punto de vista analítico puede considerarse como una función expresada como cociente de polinomios en $s=j w$

$$
H(s)=\frac{G(s)}{F(s)}
$$

donde $F(s)$ y $G(s)$ son polinomios de orden n. Las raíces del numerador proporciona los "ceros" y las del denominador los "polos" de la función transferencia. El "orden" lo determina el número de polos.

Se puede definir $h=\varepsilon / \omega_{0}$ como la constante de amortiguamiento y cuando $h=1$ se dice que el sistema está críticamente amortiguado; en estas condiciones una masa desplazada volverá a su posición de reposo en el menor tiempo posible, sin sobrepasar la línea base ni oscilar en torno a la misma. El rendimiento de los sismómetros generalmente es óptimo en condiciones cercanas a las de amortiguamiento crítico. 
En el caso (2.10), la función de transferencia en frecuencia relaciona el desplazamiento de la Tierra, $\vec{u}(t)$, con el desplazamiento de la masa del sensor, $\vec{z}(t)$. En el caso del sismómetro de la Fig. 2.1 que mide la velocidad de la masa, $\overrightarrow{\dot{z}}(t)$, la función de respuesta describe la respuesta del sensor a la velocidad del suelo, $\overrightarrow{\dot{u}}(t)$.

En general, los sismómetros pueden medir desplazamientos, velocidad 0 aceleración de la masa del sensor y se puede estar interesado en recuperar el desplazamiento, la velocidad o la aceleración del suelo. Es importante conocer cuál combinación está involucrada. Cada derivada en tiempo introduce un factor $-i \omega$ en el dominio de la frecuencia, de manera tal que, siendo todos los otros factores iguales, la velocidad y en especial la aceleración se verán enriquecidas en altas frecuencias respecto al desplazamiento. Cada derivada en tiempo aplicada al movimiento del sensor, multiplica la respuesta por $\omega$, mientras que cada derivada en tiempo aplicada al movimiento de la Tierra multiplica la respuesta por $\omega^{-1}$. En dos casos la función de transferencia es plana a lo largo de una ancha banda de frecuencias. El primero ocurre para la curva $\omega^{0}$ en frecuencias altas $\omega \gg \omega_{0}$, el segundo ocurre para la curva $\omega^{-2}$ en bajas frecuencias, representando la respuesta de un sensor de desplazamiento a la aceleración del suelo. Si definimos como $\ddot{U}(\omega)$ al espectro de aceleración, la respuesta de tal sensor a la aceleración del suelo se encuentra sustituyendo $U(\omega)=-\omega^{-2} \ddot{U}(\omega)$ en la ecuación (2.10):

$$
Z(\omega)=\frac{-1}{\omega_{0}^{2}-2 \varepsilon i \omega-\omega^{2}} \ddot{U}(\omega)
$$

de manera que, para bajas frecuencias:

$$
Z(\omega)=-\frac{1}{\omega_{0}^{2}} \ddot{U}(\omega) \text { para } \omega \ll \omega_{0}
$$

Este resultado también se puede obtener a partir de la ecuación (2.5) considerando que, para largos periodos, el término en $\vec{z}(t)$ dominará frente a los términos en $\overrightarrow{\dot{z}}(t)$ y $\vec{z}(t)$, de modo tal que:

$$
\begin{array}{rlr}
\omega_{0}^{2} \vec{z} & =-\overrightarrow{\ddot{u}} & \text { para } \quad \omega \ll \omega_{0} \\
\vec{z} & =-\overrightarrow{\ddot{u}} / \omega_{0}^{2}, & \\
& =-\overrightarrow{\ddot{u}} T_{0}^{2} /(2 \pi)^{2} &
\end{array}
$$


donde $T_{0}=2 \pi / \omega_{0}$ es el período propio del instrumento. De modo que la sensibilidad de un sensor de aceleración de largo periodo es proporcional al cuadrado del período propio del sensor.

El término sensibilidad puede ser entendido de dos maneras. En algunos casos, como la ganancia del instrumento, dada por la razón entre la tensión de salida y la velocidad de entrada, por ejemplo $1000 \mathrm{~V} / \mathrm{ms}^{-1}$. Sin embargo, este número no dice mucho acerca de la menor señal terrestre que se puede registrar, por lo que resulta más adecuado definir la sensibilidad como la menor señal que se puede resolver. Actualmente está limitada por el ruido generado por la electrónica (en el sensor o registrador), mientras que antes el factor de limitación era la ganancia en los amplificadores.

En este sentido, mediciones del desplazamiento de la masa pueden proveer sensibilidades significativas a la aceleración de la Tierra en frecuencias menores que la frecuencia de resonancia del sismómetro.

La banda de frecuencias en la que se registran las ondas sísmicas se puede dividir en dos partes a través del máximo del ruido del suelo (microsismo), que ocurre aproximadamente en períodos de 6 a $8 \mathrm{seg}$ (Fig. 1.2). Comúnmente se puede referir a registros de corto período (SP) para aquellos obtenidos en frecuencias por encima del máximo del microsismo, mientras que los registros de largo período (LP) son aquellos que se obtienen para frecuencias por debajo del mismo. Los sensores de corto período registran bien los arribos de ondas de volumen de alta frecuencia; el tiempo de estos arribos puede usarse tanto para ubicar terremotos como para realizar inversiones tomográficas para estructuras con velocidades tridimensionales. Los primeros arribos de ondas $\mathrm{P}$ utilizados para calcular los mecanismos focales se obtienen mejor en corto período; sin embargo, tanto el análisis de ondas superficiales, que permite obtener detallados estudios de las propiedades de la fuente, como el modelado de la forma de onda, requieren datos de períodos largos (15 a $100 \mathrm{seg}$ ).

Una forma de mejorar la sensibilidad instrumental para ondas sísmicas de períodos largos, incrementando el período propio del sensor, es bajando la constante del resorte o incrementando la masa. Desafortunadamente, ambas aproximaciones tenderían a incrementar el tamaño y encarecer los sismómetros de período largo frente a los de período corto. Un avance notable se produjo en 1935, cuando Lucien LaCoste inventó el resorte de longitud cero que permite sismómetros inerciales estables para períodos largos, los que hoy en día tienen períodos típicos que varían entre 15 y $30 \mathrm{seg}$. 


\section{2 - Sismógrafos modernos}

Los instrumentos modernos son más sofisticados que los sismógrafos descriptos anteriormente. Están diseñados para tener una respuesta lineal a los movimientos de la Tierra sobre un rango ancho tanto de amplitud como de frecuencias.

Una de las variables para caracterizarlos es el rango dinámico del sismógrafo, que se define como la razón entre la amplitud mayor y la menor que pueden ser registradas con exactitud.

Es deseable que los instrumentos tengan una sensibilidad por debajo de los niveles típicos del ruido de la Tierra, de modo de registrar los menores eventos detectables, mientras que permanecen en escala para los terremotos grandes. La sensibilidad para movimientos pequeños puede ser mejorada tanto por el diseño mecánico del instrumento como a través del uso de amplificadores de bajo ruido. Para sensores de movimientos fuertes, el problema es prevenir la no linealidad en la respuesta. La no linealidad mecánica puede producirse por la longitud finita de los resortes usados en el diseño.

A menudo la linealidad se obtiene en los instrumentos modernos a través del uso de los diseños de fuerza-realimentación, en los que la masa es mantenida en una posición fija. El sismógrafo registra la medida de la fuerza que se requiere para mantener la masa en reposo; esta fuerza está directamente relacionada con la aceleración de la Tierra. Ya que la masa se mueve muy poco, esto hace posible diseños mucho más compactos, incluyendo, por ejemplo, instrumentos sensibles a períodos largos que son lo suficientemente pequeños como para poder desplegarse en pozos.

El rango dinámico debe ser mantenido luego en las partes electrónica y de registración (ver 2.2.2). Los instrumentos analógicos y los sistemas digitales con resolución limitada (con digitalizadores de 12 o 16 bits) a menudo tienen insuficiente rango y se saturan para grandes amplitudes. El uso de los digitalizadores de 24-bits ha eliminado casi totalmente estos problemas y la necesidad de distintos pasos de ganancia. No obstante, incluso con los mejores diseños modernos, pocos sismógrafos de uso general pueden realmente registrar el movimiento del suelo cerca de epicentros de terremotos superficiales con magnitudes 7 o mayores, donde las aceleraciones pueden exceder $1 \mathrm{~g}$ (g valor de la gravedad). De modo que en muchas áreas urbanas se han establecido redes de instrumentos para movimientos fuertes llamados "strong motion". Estos son típicamente acelerómetros de bajo costo, de sensibilidad limitada, 
que proveen registros en escala para grandes terremotos. Son usados primariamente para proveer datos para el diseño de estructuras sismorresistentes. También proveen registros de la fuente cercana, muy útiles para usar en el modelado de la historia de la ruptura a lo largo de la superficie de falla en eventos grandes.

Los sismógrafos que proveen sensibilidades útiles sobre un amplio rango de frecuencias se llaman banda-ancha (BB). El uso de técnicas de fuerza-realimentación puede incrementar mucho el ancho de banda de los instrumentos, comparado con los diseños puramente mecánicos y ha hecho posible sismógrafos que registran movimientos con períodos de cientos de segundos hasta frecuencias de $10 \mathrm{~Hz} 0$ mayores. Esto significa que donde antes se utilizaban 2 o 3 sensores, un sensor puede hoy en día a menudo realizar el mismo trabajo, aunque aún no es posible cubrir todo el rango.

La electrónica se ha convertido en una parte importante en el diseño de los sismógrafos; para modificar la respuesta son usados amplificadores y filtros. Los datos pueden ser obtenidos localmente usando un registro en cinta, en disco rígido u otros medios de almacenamiento, o pueden ser enviados por telemetría o comunicación satelital a un lugar de registro remoto. Las investigaciones modernas en Sismología a menudo requieren una exactitud en el tiempo de 0.01 seg o mayor (fracción en el intervalo de muestreo) para poder hacer uso completo de los datos. Los errores en tiempo son mucho menos comunes en instrumentos modernos debido a la utilización de osciladores de cristal de cuarzo con poca deriva y/o a través de la sincronización con señales satelitales (GPS).

La respuesta instrumental puede ser definida en términos de la relación entre las cuentas digitales registradas en la serie de tiempo y el movimiento real de la Tierra. Se define la ganancia de un instrumento moderno como el cociente entre las cuentas y alguna medida del movimiento de la Tierra; de modo que instrumentos de alta ganancia son más sensibles que los de baja ganancia. Sin embargo, ya que la sensibilidad de los sismógrafos depende de la frecuencia, el concepto de ganancia para una frecuencia dada es poco representativo. Una descripción más completa queda dada a través de la función de transferencia $T(\omega)$ (ver 2.11), que especifica la respuesta en amplitud y fase continuamente como una función de la frecuencia. La respuesta instrumental puede también ser descripta por la función de respuesta impulsiva, que muestra la salida del sismógrafo en el dominio del tiempo a partir de la entrada de una función impulsiva delta de Dirac. En general, esta función se va a 
aproximar más a la función delta a medida que el instrumento se torna más bandaancha.

Además del diseño del instrumento, es muy importante la certeza de la información de la calibración. Un sismograma no es muy útil para los investigadores si no se cuenta con información de la precisa ubicación del instrumento, la orientación de los sensores horizontales, la frecuencia de muestreo, el tiempo de la primer muestra y la función de respuesta completa del instrumento.

Normalmente se necesita una red de estaciones sismológicas para localizar terremotos y realizar otras investigaciones. Instalar una red es principalmente una cuestión de comunicación. Este campo está en rápido desarrollo y probablemente cambiará completamente nuestro concepto de realización de redes. En un principio, una red era a menudo un sistema que permitía vincular estaciones a través de radiotelemetría analógica a distancias de algunos cientos de kilómetros, enviando datos analógicos desde las estaciones de campo a un registrador digital central. Mientras que este tipo de redes aún se siguen realizando usando transmisión digital, la tendencia es que las estaciones de campo se tornen nodos de computadoras independientes, los que, por software, pueden ser vinculados en redes sísmicas virtuales, así llamados para distinguirlos de las redes físicas clásicas. De modo que, en las redes virtuales, los sistemas de comunicación se encuentran por diseño, separados de las estaciones sísmicas en sí mismas y pueden estar basados en canales de comunicación de radio, satélites o de datos públicos.

Una red sismológica virtual reside entonces en estaciones seleccionadas entre muchas estaciones conectadas a la red global de comunicaciones o al sistema telefónico público. Las estaciones remotas deben ser capaces de detectar los eventos y registrar localmente; los datos no son normalmente enviados al sistema central de registración en tiempo real. Las estaciones remotas deben tener la capacidad de comunicación de dos maneras: el sistema central debe poder conectarse manual o automáticamente a la misma, bajar datos definidos o continuos y hacer evaluaciones inteligentes de eventos posibles.

En la Fig. 2.3 se observa una comparación entre las respuestas en frecuencia de sismógrafos BB modernos con respecto a diseños anteriores para diversas redes (Shearer, 1999). La red original IDA (International Deployment of Accelerometers) fue la primer red sismológica global digital. IDA usaba gravímetros diseñados para registrar los modos normales de la Tierra en períodos muy largos y registraban una muestra cada 10 seg. 


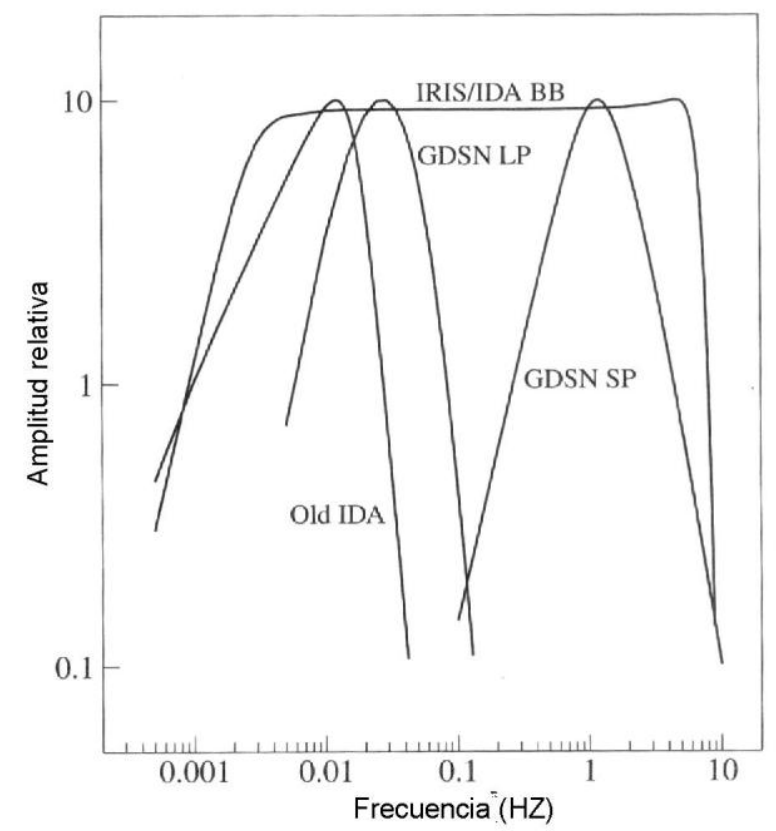

Figura 2.3: Funciones de transferencia en velocidad para cuatro instrumentos de componente vertical (estación antigua IDA, canales de corto y largo período para una estación GDSN y una estación IRIS/IDA), extraído de Shearer, 1999.

La red GDSN (Global Digital Seismograph Network) comenzó a tener datos disponibles hacia finales de la década del 70. Un canal de largo período en GDSN registraba 1 muestra por segundo y el canal de corto período registraba 20 muestras por segundo. Las funciones de respuesta de GDSN fueron diseñadas para evitar el máximo del ruido entre 5 y 8 seg de período. Los instrumentos BB comenzaron a ser desplegados en cantidad hacia finales de los 80 y principios de los 90; estas estaciones BB que fueron desplegadas en redes tales como IRIS tienen una respuesta lineal en una banda ancha de frecuencias.

La red IRIS/GSN (Incorporated Research Institutions for Seismology/Global Seismic Network) consiste en más de 120 estaciones sismológicas BB, a las que se puede acceder por modem y/o Internet. En el centro de administración de datos en Washington un software de dominio público, SPYDER, que corre bajo Unix/linux, permite recuperar datos de estaciones GSN seleccionadas basado en la determinación preliminar de epicentros del NEIC (National Earthquake Information Center) en Boulder, Colorado. Esta detección de eventos no es parte del sistema Spyder, que se ha instalado en varios sitios para uso local o global y únicamente trabaja con estaciones del tipo GSN. Este sistema global es un ejemplo típico de una red sismológica virtual. 


\subsubsection{Sensores}

Se ha visto que un sensor sísmico es un instrumento para medir el movimiento del suelo cuando es sacudido por una perturbación. Este movimiento es dinámico y el sensor sísmico o sismómetro también debe dar una variable dinámica física relacionada a este movimiento. En los primeros sismómetros, esta variable era el desplazamiento de una pluma representando el movimiento amplificado del suelo. En nuestros días, la salida física del sismómetro es siempre una tensión. Idealmente, podemos considerar al sismómetro como una caja negra cuya entrada es el movimiento del suelo (representado por una variable cinemática: desplazamiento, velocidad o aceleración) y su salida es una tensión - o movimiento de una pluma, en equipos antiguos.

Actualmente, los sismómetros pueden medir sólo velocidades 0 desplazamientos asociados a valores no nulos de aceleración. La respuesta para velocidades y desplazamientos del suelo se aproxima a cero a medida que las frecuencias decrecen. Esta es una característica esencial de los sismómetros inerciales y no puede ser superada. Otro tipo de sensores miden deformación y en este caso ellos pueden responder a desplazamientos relativos estáticos también, ya que no están basados en la inercia. Los primeros sensores sensibles a bajas frecuencias fueron hechos con sofisticados sistemas mecánicos, los que con toda clase de artificios eran capaces de tener una baja frecuencia de resonancia. Sin embargo no era posible hacer sensores con una frecuencia de resonancia estable menor que $0.03 \mathrm{~Hz}$. Actualmente, los sensores únicamente mecánicos se construyen sólo para frecuencias de resonancia de aproximadamente $1.0 \mathrm{~Hz}$ (sensores de corto periodo), mientras que los sensores que puedan medir frecuencias menores se basan en el Principio de Fuerza Balanceada (FBP) que mide la aceleración directamente. Los Acelerómetros de Fuerza Balanceada (FBA), Fig. 2.4, tienen una bobina de realimentación, que puede ejercer una fuerza igual y opuesta a la fuerza de inercia debida a la mayor aceleración que se quiera medir. Un transductor de desplazamiento envía una corriente a esta bobina a través de un resistor $R$ en un lazo de realimentación negativo. La polaridad de la corriente es tal que se opone a cualquier movimiento de la masa y va a lograr que la masa no se mueva con respecto al soporte. Una pequeña aceleración permanente en la masa entonces, resultará en una pequeña corriente permanente y una gran aceleración necesitará una gran corriente. La corriente es entonces linealmente proporcional a la aceleración del suelo, de modo 
que una medición directa de la aceleración queda dada por el voltaje sobre la resistencia.

El principio de FBA es actualmente el corazón de prácticamente todos los sensores modernos de movimientos fuertes (strong motion) y de banda ancha. Conectando e integrando el circuito, en el lazo de realimentación, o después, el sensor entrega una tensión linealmente proporcional a la velocidad. Sin embargo, debido al principio de inercia, debe haber un límite en las frecuencias bajas (la velocidad es cero para las frecuencias cero) y este límite está dado por las cualidades mecánicoeléctricas del sensor.

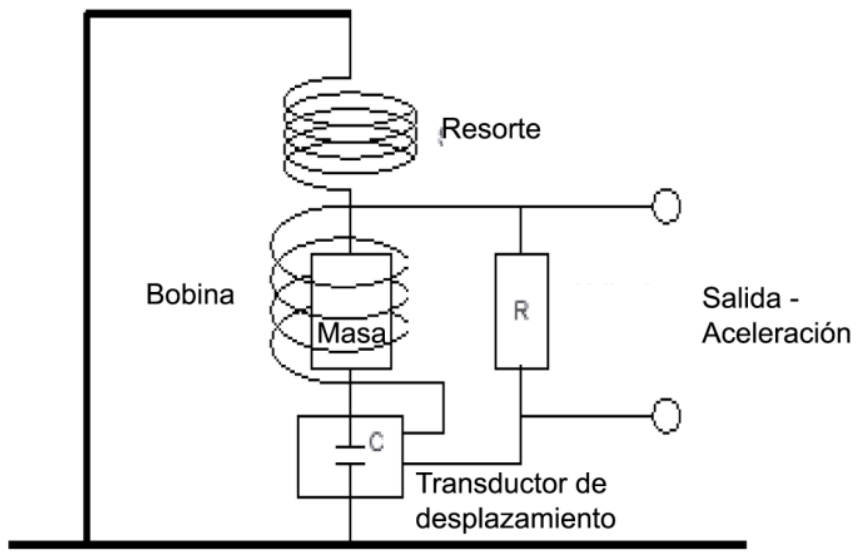

Figura 2.4: Esquema de un acelerómetro de fuerza balanceada. El transductor de desplazamiento normalmente utiliza un capacitor C, cuya capacitancia varía con el desplazamiento de la masa. Una corriente proporcional al transductor de desplazamiento forzará la masa a permanecer estacionaria respecto al soporte.

En general, el mejor sensor de banda ancha tiene un límite de aproximadamente $0.0025 \mathrm{~Hz}$, mucho menor que el que siempre fue posible con sensores puramente mecánicos. El principio FBA también tiene la ventaja de obtener sensores lineales, con un alto rango dinámico ya que la masa prácticamente no se mueve. Actualmente, los mejores sensores tienen un rango dinámico de $10^{6}-10^{8}$.

En resumen, los sensores mecánicos son los más usados cuando la frecuencia natural está por encima de $1 \mathrm{~Hz}$, mientras que los sensores de tipo FBA son usados como acelerómetros y sensores banda ancha.

Esto significa que un gran número de sensores son cajas negras de componentes mecánicos y electrónicos altamente complejos con los cuales el usuario puede hacer muy poco. Sin embargo, es aún posible hacer una serie de tests simples para chequear la funcionalidad del instrumento. 


\subsubsection{Registradores}

Durante los últimos 20 años ha habido un rápido desarrollo de la registración digital, que ha permitido acompañar el adelanto de los sismómetros y obtener la capacidad de registrar las señales actuales de los sismómetros sin perder calidad.

Los registros analógicos aún se continúan utilizando ya que ellos permiten una mirada rápida y fácil de los sismogramas. Sin embargo, el rango dinámico en este caso es muy limitado (a lo sumo 200 en contraste a $10^{8}$ en los digitales). Los registradores analógicos son muy costosos tanto para comprar como para operar, de modo que ya prácticamente no se instalan más. Además, pocos de estos registradores entregan una señal analógica que sirva de entrada para los registradores modernos, de modo que se debe instalar un convertidor analógico-digital. El desarrollo de sensores de gran rango dinámico y de banda ancha no habría mejorado mucho la Sismología si no hubieran también mejorado las técnicas de registración.

Convertir la señal analógica a digital significa convertir una señal que varía continuamente, en muestras discretas de esa señal analógica.

El digitalizador convierte entonces un voltaje en un número, lo mismo que realiza un voltímetro digital. La diferencia es que se debe hacer muchas veces por segundo en una señal de amplitud variable. Hay dos aspectos importantes en el digitalizador: el intervalo de muestreo (paso en la dirección del tiempo) y la resolución (paso en la dirección de la amplitud), que corresponde a un paso en la salida numérica del digitalizador.

Los digitalizadores se clasifican usualmente como de 12 bits, 16 bits o 24 bits que representa el número de valores discretos que usa. Un convertidor de 12 bits tiene $2^{12}$ niveles y un convertidor de 24 bits, $2^{24}$ niveles $0 \pm 2^{11}$ y $\pm 2^{23}$ respectivamente. Esto corresponde a rangos dinámicos de 2048 y $8.410^{6}$ respectivamente. Actualmente la mayoría de los digitalizadores están basados en convertidores analógicos-digitales (CAD) de 24 bits; sin embargo, pocos tienen una performance de 24 bits, ya que las entradas más pequeñas entregan un número de cuentas significativamente diferente de cero. De manera que los digitalizadores no están de acuerdo a los mejores sensores ya que usualmente sólo alcanzan un rango dinámico de 21-22 bits.

El paso en la dirección de la amplitud depende de la resolución del digitalizador y es el menor paso que puede resolver. Los mejores digitalizadores pueden resolver usualmente $100 \mathrm{nV}$ que idealmente corresponde al número 1. De modo que si se asume que el número 1 fuera del digitalizador corresponde a la señal real de entrada 
(sin ruido del digitalizador), entonces el mayor número corresponde directamente al rango dinámico.

Si se necesita registrar una señal con contenido de alta frecuencia, es necesario incrementar la frecuencia de muestreo (aunque el rango dinámico usualmente se deteriora levemente, con una frecuencia de muestreo mayor) ya que se debe muestrear al menos a dos veces el paso de la frecuencia de la señal que se quiere registrar (frecuencia de Nyquist).

Los digitalizadores y registradores son a menudo una unidad única aunque físicamente pueden ser dos partes separadas. La registración se hace actualmente en discos rígidos, aunque algunas unidades usan memoria de estado sólido.

Ya que se pueden utilizar grandes discos rígidos para la registración, la memoria de los registradores ya no es más un problema y actualmente es fácil trabajar con registración continua. No obstante, casi todos los registradores también tienen la facilidad de guardar sólo los segmentos de tiempo que contienen eventos reales y el sistema se dice que funciona por disparo. Esto significa que un programa monitorea continuamente las señales entrantes para decidir cuando ocurre un evento real.

\section{3 - Instalación}

La instalación de una estación no significa simplemente poner el sensor y registrador en algún lugar y comenzar a registrar; aunque podríamos tener datos, estarían tan contaminados que serían inservibles. Lo que se quiere es tener un sensor que sea tan insensible a las fuentes de ruido artificiales (humanas y ambientales) como sea posible, de manera tal que la sensibilidad para señales generadas por terremotos sea alta. En otras palabras, la estación debería estar tan lejos de los océanos y del hombre como sea posible. Esta pretensión entra en conflicto con consideraciones prácticas tales como acceso y costos, de modo que siempre se debe optar por un compromiso.

Actualmente las estaciones pueden tener distintos diseños, aunque en general se instalan en sitios donde haya facilidades de comunicación. Además necesitan energía ya sea obtenida de línea de potencia pública o a través de energía no convencional, siendo ésta una de las condiciones más importantes para lograr un buen funcionamiento. 
El aspecto más crítico de la puesta en funcionamiento de una estación sismológica es la instalación del sensor, en particular para sensores banda ancha.

Cuando se instalan nuevas estaciones, éstas pueden ser parte de una red local o regional, o cada una puede ser una estación única, que puede ser parte de una red global como las estaciones IRIS. Una estación que forme parte de una red local obviamente no puede estar ubicada en cualquier lugar, ya que la red debe tener una configuración óptima teniendo en cuenta los eventos mejor localizados en la región.

\subsubsection{Ubicación geográfica.}

Estudiando un mapa se pueden tener ubicaciones ideales, a menudo remotas, las que pueden ser severamente cuestionadas por consideraciones prácticas. Las mas importantes que se deben tener en cuenta son:

Potencia: la potencia requerida por un equipo puede variar entre $1 \mathrm{~W}$ a más de 100 W dependiendo del tipo de estación. Las estaciones de alta potencia necesitan un acceso a una línea pública mientras que las estaciones de baja potencia la pueden obtener con celdas solares, en cuyo caso se deben realizar cuidadosas consideraciones en el tamaño de las baterías y las celdas solares a usar. Este factor es crítico y a veces es preferible comprometer otros aspectos de la instalación de la estación en función de poder utilizar una línea de potencia pública.

Comunicación: si se requiere que la estación transmita por radio, a menudo debe ser ubicada cerca de las cumbres de las montañas. Las comunicaciones por satélite no tienen esta limitación, pero usan más potencia. Las líneas de comunicación terrestre requieren que la estación esté cerca de la línea de teléfono público. Las líneas telefónicas a menudo no están disponibles y el costo puede ser elevado.

Ruido: la estación debe estar alejada de fuentes de ruido, en particular antropogénicas.

Seguridad: Desafortunadamente, el robo y el vandalismo no son inusuales y algunas áreas son peores que otras. De modo que además de hacer construcciones sólidas, la ubicación geográfica puede ser importante para evadir estos problemas.

Acceso: las ubicaciones ideales podrían requerir un helicóptero para acceder. Ya que la mayoría de los sismólogos no podrían afrontar este costo, el acceso fácil a un sitio es esencial para asegurar el mantenimiento a largo plazo.

Clima: áreas con condiciones de humedad muy altas, fríos o calores extremos o con una alta probabilidad de tormentas eléctricas deberían evitarse. 
Topografía: en general se trata de evitar topografía compleja, que podría modificar las formas de onda, aunque esto entra en conflicto cuando se instalan estaciones en la cima de montañas para tener mejor comunicación.

Geología: es bien conocido que un sensor en suelo blando es más ruidoso que un sensor en roca sólida, de modo que el suelo blando debería ser evitado.

Los requerimientos mencionados anteriormente son todos conflictivos ya que una estación ideal debería situarse en una área remota sin potencia, comunicaciones o seguridad. Ya que para la mayoría de las organizaciones que operan estaciones sismológicas, es usualmente más fácil obtener fondos para la instalación inicial que para su operación a lo largo del tiempo, el compromiso primario debería ser asegurar bajo costo y estabilidad operacional por un largo término,. Esto a menudo significa que el nivel de ruido será más alto que el ideal y se registrarán menos terremotos, sin embargo ya que la continuidad de operación será mayor, la cantidad total de datos adquiridos podría ser comparable al monto de datos de una estación con operación discontinua. Considerando el crecimiento exponencial del número de estaciones sismológicas, no sería una gran pérdida registrar un poco menos en un lugar particular, si se obtiene un registro continuo con buena información.

\subsubsection{Selección del sitio y campaña de ruido sísmico.}

Una estación sismológica ubicada en basamento siempre se espera que sea menos ruidosa que una estación sobre sedimentos blandos, incluso si no hay en las cercanías fuentes de ruido. Sin embargo, puede haber fuentes de ruido desconocidas o la geología puede ser diferente de lo que parece ser en superficie. Lo que parece ser el lecho de rocas, podría ser un gran canto rodado.

Antes de realizar la selección final del lugar para la estación sismológica, se debe llevar a cabo un estudio de ruido. El ruido por encima de $1 \mathrm{~Hz}$ está generado en su mayoría en el campo cercano, mientras que el ruido de baja frecuencia se podría originar lejos. Esto significa que una estación de banda ancha que registra principalmente señales de baja frecuencia podría operar cerca de una ciudad si las condiciones geológicas son favorables.

La campaña de ruido consiste en registrar continuamente por ejemplo durante 24 horas, o más si es posible. Entonces es posible tomar intervalos de 10 min (por ejemplo) para una estación BB y realizar el promedio del espectro de ruido, que puede entonces compararse con las curvas de nivel de ruido (ver Fig. 1.2). De esta forma se puede evaluar si el nivel de ruido promedio es satisfactorio. El registro debería hacerse 
con el mismo tipo de sensor que se espera instalar a efectos de cubrir la misma banda de frecuencias. Esto podría ser difícil en la práctica ya que el sensor podría no estar disponible. En lugares para registración de banda ancha, es también difícil realizar una instalación temporaria con suficiente estabilidad de temperatura para obtener resultados útiles.

En todos los casos, el principal objetivo de una campaña de ruido es investigar las fuentes cercanas ya que el ruido de baja frecuencia es normalmente constante sobre una gran área geográfica. El registro puede hacerse con un sismómetro de corto período con la amplificación apropiada, el que dará una buena resolución del ruido (microsismo) debajo de $0.1 \mathrm{~Hz}$. Es importante que la ganancia se use lo suficientemente alta para obtener una buena resolución del ruido, de modo que la ganancia debería ser sustancialmente mayor que la usada para registración normal, donde el propósito es tener el mejor rango dinámico para registrar terremotos.

Idealmente la registración debería ser hecha durante el día y la noche y el invierno y verano, sin embargo, esto pocas veces se realiza. En la mayoría de los casos debería ser posible registrar continuamente durante al menos 24 horas para detectar fuentes locales de ruido periódico. Esto significa que además del análisis espectral se debe inspeccionar el registro continuo ya que podrían haber pequeñas perturbaciones impulsivas, que no se verían en el espectro o que podrían aumentar el nivel espectral.

Para frecuencias debajo de $0.1 \mathrm{~Hz}$ (usando un sensor $\mathrm{BB}$ ), es mucho mas difícil realizar una buena evaluación de ruido sin una cuidadosa instalación. No hay mucha diferencia del nivel de ruido fundamental de largo período sobre roca sólida en distintos lugares cercanos, ya que las fuentes de ruido de baja frecuencia generalmente son lejanas y la mayor parte del ruido de baja frecuencia que se registra, originado cerca de la superficie, es debido a cambios de presión y temperatura que induce cambios de inclinación en el suelo.

En otras palabras, hacer una medición de ruido con un sensor BB nos dice muy poco más que las mediciones realizadas con un sensor de $1 \mathrm{~Hz}$, a no ser que se pueda hacer una buena instalación sobre roca sólida con condiciones estables de temperatura (cambios de $1 \mathrm{grado} /$ día). En cualquier caso, el ruido de período muy largo está mucho más influenciado por la profundidad y tipo de instalación que por la ubicación geográfica. 


\subsubsection{Instalación de la estación sismológica.}

Asumiendo que se ha elegido un sitio apropiado donde se han satisfecho todos los requerimientos básicos de ruido, facilidades físicas, etc., la instalación consiste en instalar el sensor y posiblemente el registrador y/o el equipo de transmisión. La instalación dependerá del tipo de sensor y de registración local o remota que se realizará.

Por convención, los sensores horizontales en superficie siempre deben orientarse NS y EO de modo tal que un movimiento $\mathrm{N}$ o $\mathrm{E}$ dé una señal positiva. $\mathrm{El}$ modo más común de orientar los sensores es usando un compás magnético, corrigiendo por la declinación magnética. La orientación de los sensores debería estar dentro de un error de \pm 2 grados con respecto a las direcciones mencionadas. La componente vertical se orienta utilizando un nivel de burbuja, a veces incorporado al sensor.

Usualmente los sensores de corto período y acelerómetros no requieren aislamiento térmico ya que son poco sensibles a los cambios de temperatura. En cambio, los sensores BB requieren un aislamiento térmico muy bueno.

Es importante instalar los sensores sobre basamento, si es posible. Si esto no es posible, siempre es una ventaja poner el sensor algunos metros debajo del nivel del suelo. Si la instalación se realiza en profundidad, por lo mencionado anteriormente en 2.3.2, se elimina ruido de baja frecuencia ocasionado principalmente por cambios de temperatura.

Los sensores de corto período $(1 \mathrm{~Hz})$ son instalados a veces incluso en recintos de concreto sobre el suelo los que también sirven como alojamiento del equipamiento y como una instalación segura. Estos son los sensores de instalación más común, tanto de una como de tres componentes. 


\section{Capítulo 3 \\ CÁLCULO DEL RUIDO}

\subsection{Señales Sísmicas}

La señal irradiada de una fuente sísmica, sea una explosión o una ruptura transversal, es usualmente una función escalón con mayor o menor complicación en su desplazamiento o un impulso en velocidad de dirección finita de milisegundos hasta unos pocos minutos a lo sumo.

De acuerdo al Teorema de Fourier, una función transitoria $f(t)$ arbitraria en el dominio del tiempo puede ser representada por una función equivalente $F(\omega)$ en el dominio de la frecuencia, la transformada de Fourier de $f(t)$.

$$
\begin{gathered}
f(t)=\frac{1}{2 \pi} \int_{-\infty}^{\infty} F(\omega) e^{i \omega t} d \omega \\
F(\omega)=\int_{-\infty}^{\infty} f(t) e^{-i \omega t} d t=|F(\omega)| e^{i \phi(\omega)}
\end{gathered}
$$

(la convención de signos puede cambiar, e.g. $e^{-i \omega t}$ en (3.1) y $e^{i \omega t}$ en (3.2) en estudios de propagación de ondas, para asegurar que el vector número de onda sea positivo en la dirección de propagación de la onda)

$$
\begin{gathered}
|F(\omega)|=A(\omega) \text { es la densidad de amplitud espectral en }[\mathrm{m} / \mathrm{Hz}] \\
w=2 \pi f \text { la frecuencia angular (con la frecuencia } f \text { en }[\mathrm{Hz}] \text { ) } \\
\phi(\omega) \quad \text { la fase espectral [en grados, } \mathrm{rad} \text { o } 2 \pi \mathrm{rad}]
\end{gathered}
$$

\section{2 - Ruido sísmico}

\subsubsection{Introducción}

Mientras que los componentes armónicos de señales sísmicas transitorias irradiadas desde fuentes localizadas de duración finita son coherentes en sus relaciones de fase, definidas por la fase espectral, éste no es el caso del ruido sísmico ambiental. Este último está causado por fuentes diversas, no espaciadas uniformemente, diferentes, generalmente no relacionadas y a menudo continuas.

El ruido sísmico en suma es un proceso estocástico estacionario. 
Lo mismo ocurre con el ruido electrónico del instrumental y el movimiento browniano (térmico) de las masas.

Debido a la naturaleza estocástica del ruido, la ec. (3.2) no converge, entonces no se pueden calcular la densidad de amplitud espectral o la fase espectral; se determina en consecuencia la densidad de potencia espectral $P(\omega)$.

$P(\omega)$ es la transformada de Fourier de la correlación:

$$
\begin{aligned}
p(\tau) & =<f(t) f(t+\tau)>\quad<>\text { :promedio sobre el tiempo } t \\
P(\omega) & =\int_{-\infty}^{\infty} p(\tau) e^{-i \omega \tau} d \tau
\end{aligned}
$$

las unidades de $P(\omega)$ serán: [ $\left.\mathrm{m}^{2} / \mathrm{Hz}\right]$ para un registro de desplazamiento,

y

$$
\begin{aligned}
& {\left[(\mathrm{m} / \mathrm{s})^{2} / \mathrm{Hz}\right]=\left[\mathrm{m}^{2} / \mathrm{s}^{2} / \mathrm{Hz}\right] \quad \text { para un registro de velocidad }} \\
& {\left[\left(\mathrm{m} / \mathrm{s}^{2}\right)^{2} / \mathrm{Hz}\right]=\left[\mathrm{m}^{2} / \mathrm{s}^{4} / \mathrm{Hz}\right] \quad \text { para un registro de aceleración }}
\end{aligned}
$$

El movimiento oscilatorio del suelo $x(t)$ generado a partir del ruido sísmico (así como también de los términos armónicos de una señal transitoria) pueden ser aproximados por ondas sinusoidales $x(t)=a_{d} \operatorname{sen}(\omega t)$, donde $a_{d}$ es la amplitud del desplazamiento. Entonces, cuando se pasa de desplazamiento a velocidad $(d x / d t) 0$ aceleración $\left(d^{2} x / d t^{2}\right)$, obtenemos las respectivas amplitudes de velocidad: $a_{v}=a_{d} \omega$ y de aceleración $a_{a}=a_{d} \omega^{2}$.

Conociendo la densidad de potencia espectral del desplazamiento, $P_{d}(\omega)$, podemos calcular los valores de la densidad de potencia espectral de la velocidad y de la aceleración:

$$
\begin{gathered}
P_{v}(\omega)=P_{d}(\omega) \cdot \omega^{2}=4 \pi^{2} f^{2} P_{d} \\
P_{a}(\omega)=P_{d}(\omega) \cdot \omega^{4}=16 \pi^{4} f^{4} P_{d}=4 \pi^{2} f^{2} P_{v}=P_{v} \cdot \omega^{2}
\end{gathered}
$$

Las señales sísmicas relativas a la potencia del ruido $\left(\frac{a_{2}}{a_{1}}\right)^{2}$, como en acústica, donde las $a$ representan amplitudes, se expresan a menudo en unidades de decibeles $[\mathrm{dB}]$.

La potencia en $\mathrm{dB}$ es $10 \log \left(\frac{a_{2}}{a_{1}}\right)^{2}=20 \log \left(\frac{a_{2}}{a_{1}}\right)$. 
Cuando se expresa la densidad de potencia espectral en unidades de $\mathrm{dB}$ referidas a $1\left(\mathrm{~m} / \mathrm{s}^{2}\right)^{2} / \mathrm{Hz}$, la ec. (3.5) puede ser escrita como:

$$
P_{a}[d B]=10 \log \left(P_{a}(\omega) / 1\left(m / s^{2}\right)^{2} / H z\right)
$$

Peterson (1993) ha representado un nuevo modelo global de ruido (NNM) en estas unidades. Éste representa las envolventes superior (NHNM) e inferior (NLNM) de una compilación acumulativa de las densidades de potencia espectrales en aceleración, determinadas para períodos ruidosos y quietos, de 75 estaciones digitales del mundo. Estas estaciones se encontraban operando bajo condiciones muy diferentes, situadas tanto lejos de los océanos como en islas o cercanas a áreas costeras, ubicadas sobre una gran variedad de suelos de tipo rocoso, a diferentes distancias de las áreas urbanas y equipadas con sismógrafos de diferentes rangos de ancho de banda, desde corto período a ultra largo período en el rango de $10^{-2}$ a $10^{5} \mathrm{~s}$ y con sistemas de adquisición de datos operados con diferente resolución y muestreos entre 0,01 a 100 mps. Peterson cuidadosamente observó los registros, eliminó los eventos sísmicos y otros fenómenos transitorios, pero también momentos de tormentas microsísmicas intensas, identificó rangos, donde el ruido instrumental pudiera dominar el ruido y promedió los espectros de tres registros independientes en períodos de tiempo muy quietos y muy ruidosos. De esta manera se aseguró que sus modelos representaran los límites superior y inferior de la densidad de potencia en aceleración del ruido "típico" , más que el máximo y mínimo ruido. Las envolventes del ruido fueron aproximadas por una secuencia de ajustes lineales y presentadas en unidades de $\mathrm{dB}$ referidas a $1\left(\mathrm{~m} / \mathrm{s}^{2}\right)^{2} / \mathrm{Hz}$ como ya se ha mencionado.

Si se sustituye el período $T=\frac{1}{f}$ en [s] por la frecuencia $f$ en (3.4) y (3.5) se obtiene:

$$
\begin{gathered}
P_{v}[d B]=P_{d}[d B]+20 \log (T / 2 \pi) \\
\text { y } \quad P_{a}[d B]=P_{d}[d B]+40 \log (T / 2 \pi)=P_{v}[d B]+20 \log (T / 2 \pi)
\end{gathered}
$$

de modo que para el período $T=2 \pi, P_{a}=P_{v}=P_{d}$ (Fig. 3.1).

Además, $\left(P_{d}-P_{a}\right)=2\left(P_{v}-P_{a}\right)=$ cte, para un período dado, negativo para $T<2 \pi$ y positivo para $T>2 \pi$. 


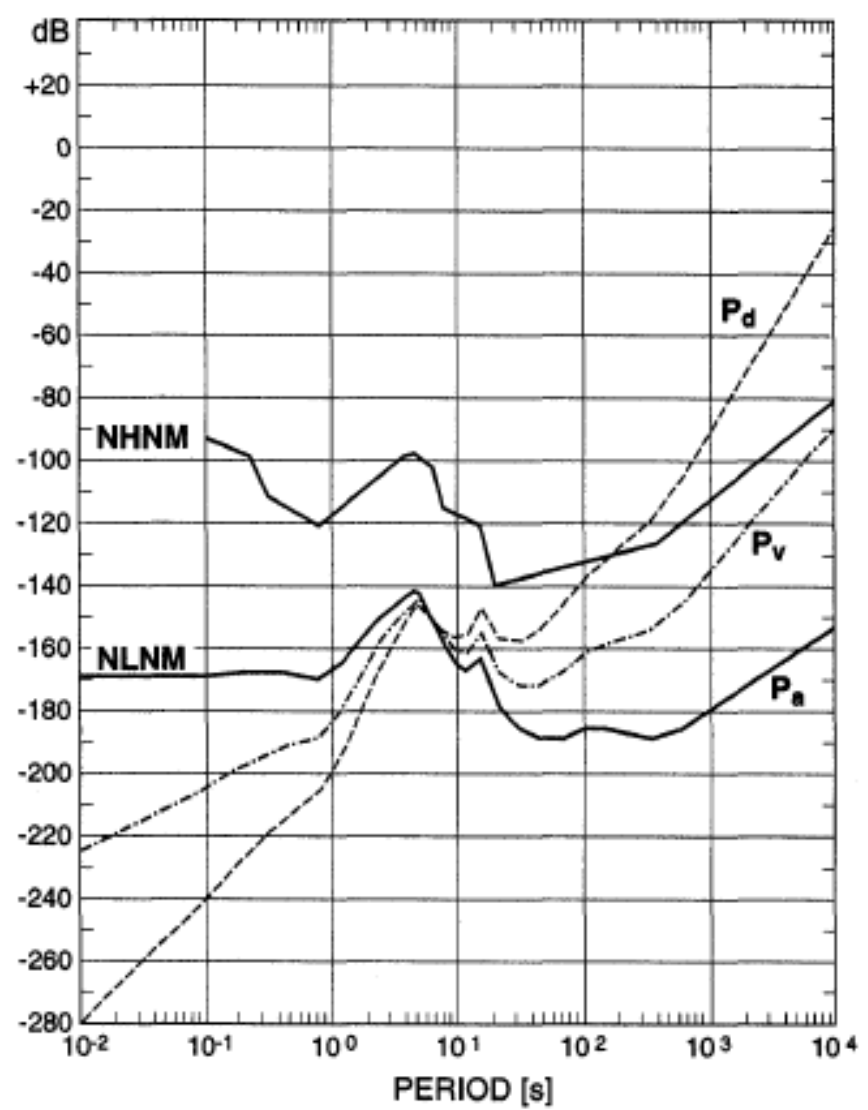

Figura 3.1: Curvas de las densidades de potencia espectral en aceleración, velocidad y desplazamiento. Extractado de Bormann, 2002.

\subsubsection{Medición del ruido}

Prácticamente en todos los lugares del mundo los sismogramas muestran alguna clase de ruido cuando la ganancia se aumenta; se puede observar que domina un ruido cuasi-armónico, llamado ruido microsísmico, y que hay también ruido sísmico significativo en otras bandas de frecuencia. De modo que, obviamente, el nivel de ruido debe ser especificado a frecuencias diferentes.

Si se grafica el ruido sísmico con diferentes bandas de filtrado para una estación cercana al océano, la traza no filtrada claramente mostrará un alto nivel de ruido de baja frecuencia $(0,3-0,5 \mathrm{~Hz})$ generado por el mar.

Intuitivamente, el modo más simple de medir el ruido sería hacerlo a través de los desplazamientos de la Tierra en bandas de diferentes frecuencias, graficando la amplitud como una función de la frecuencia o el período. Este fue el modo, de hecho, en que se hizo antes del uso de registros digitales.

El uso de filtrado y mediciones en el dominio del tiempo del ruido presenta dos problemas: 
1) el ancho de banda utilizado es arbitrario

2) se toman valores promedios sobre largos intervalos de tiempo,

Estos dos problemas se resuelven presentando el ruido en el dominio espectral.

El problema del ancho de filtro se puede observar si se toma la misma señal, filtrada con filtros cada vez más angostos, la amplitud de la señal decrecerá a medida que se apliquen los filtros, ya que menos y menos energía quedará dentro de la banda de los mismos. De modo que para hacer mediciones en el dominio del tiempo, los valores de ruido pueden solo ser comparables si se usa el mismo ancho de banda para el filtro.

Con datos digitales, es posible hacer análisis espectral, y entonces fácilmente obtener el nivel de ruido en todas las frecuencias en una operación simple.

Actualmente se ha tornado como convención representar el espectro de ruido en una estación sismológica, como la densidad de potencia espectral en aceleración $P_{a}(w)$, expresado en unidades de $\mathrm{dB}$ referidas a $1\left(\mathrm{~m} / \mathrm{s}^{2}\right)^{2} / \mathrm{Hz}$ de acuerdo a la ec. (3.8), y referirlo a las curvas de Peterson, NLNM y NHNM, ya mencionadas (fig. 3.1).

Ya que la densidad de potencia espectral puede ser definida de diferentes maneras, es importante que la definición usada sea idéntica a la usada originalmente por Peterson, para poder comparar los espectros.

\subsubsection{Relación entre mediciones de densidad de potencia espectral y de amplitud}

Las curvas de ruido de Peterson y el modo de representarlas han standardizado la manera de representar el ruido sísmico. Sin embargo, observando esas curvas, es difícil poder relacionarlas a algo físico.

Cuál es el significado físico de las curvas de Peterson?

Las curvas de ruido realizadas en el dominio del tiempo pueden ser relacionadas directamente al sismograma, mientras que las curvas de Peterson no lo hacen, ya que reflejan una medición en el dominio de la frecuencia. Sin embargo como se verá, bajo ciertas condiciones, es entonces posible ir de unas hacia las otras.

El problema es cómo relacionar una amplitud espectral a una dada frecuencia con una amplitud en el dominio del tiempo en una dada banda de frecuencia. 
La amplitud media cuadrática RMS de una señal en un intervalo de tiempo 0-T, se define como:

$$
a_{R M S}^{2}=\frac{1}{T} \int_{0}^{T} a(t)^{2} d t
$$

Entonces, la potencia promedio de la señal en el intervalo de tiempo es igual a $a_{R M S}^{2}$. Esta se puede calcular a partir de la densidad de potencia espectral (Teorema de Parseval) como:

$$
a_{R M S}^{2}=\int_{f_{1}}^{f_{2}} P(w) d f=P\left(f_{2}-f_{1}\right)
$$

considerando que la densidad de potencia espectral es aproximadamente una constante $P$ en el rango de frecuencias $f_{1}$ a $f_{2}$, que no es irrazonable si el filtro es angosto. En el caso general, $P$ podría representar el valor promedio de $P(w)$ en esta banda de frecuencias.

Es importante que $P(w)$ sea la densidad de potencia espectral normalizada y que la potencia represente la contribución total a $w$ tanto para frecuencias positivas como negativas. $\mathrm{Si}$, como es de práctica usual, la densidad de potencia espectral se calculara usando solamente las frecuencias positivas, $a_{R M S}^{2}$ deberá calcularse como $2 P\left(f_{2}-f_{1}\right)$.

Los valores de densidad de potencia dados por el Nuevo Modelo Global de Ruido de Peterson (1993) contienen el factor 2, o dicho en otras palabras representan la densidad de potencia total.

Bajo estas condiciones, se tiene entonces una relación entre la amplitud media cuadrática y la densidad de potencia espectral en una banda angosta de frecuencia:

$$
a_{R M S}=\sqrt{P\left(f_{2}-f_{1}\right)} .
$$

Hay entonces un modo simple de relacionar la densidad de potencia espectral a las amplitudes, como se ven en un sismograma; sin embargo, la última relación da la amplitud media cuadrática (RMS).

Estadísticamente, hay un $95 \%$ de probabilidad que el pico instantáneo de amplitud de una ondícula tomada al azar con una distribución de amplitudes Gaussiana se encuentre en el rango de $2 a_{R M S}$; Peterson (1993) mostró que tanto las amplitudes del ruido de banda ancha como de largo período siguen muy de cerca la distribución Gaussiana de probabilidad. En el caso de filtros de banda angosta, los 
valores promedio de las amplitudes pico son el 1,25 $a_{R M S}$ (Bormann, 2002). De modo que, para tener un verdadero promedio de las amplitudes pico en un sismograma, se puede usar un factor de aproximadamente 1,25. Entonces, se establece la relación entre los valores de la potencia espectral y las amplitudes pico promedio como:

$$
a=1,25 a_{R M S}=1,25 \sqrt{P\left(f_{2}-f_{1}\right)} .
$$

La banda de frecuencias depende del instrumento, fundamentalmente si es analógico, ya que para datos digitales el usuario puede seleccionar el filtro.

Una forma común de especificar la banda del filtro es usando el término octava de filtro. Un filtro de n- octavas tiene límites:

$$
\frac{f_{2}}{f_{1}}=2^{n} \quad,
$$

por ejemplo, un filtro de $1 / 2$ octava tiene un límite dado por $f_{1}$ y entonces: $f_{2}=f_{1} 2^{1 / 2}$ (e.g. 1-1.42 Hz).

Muchos de los sismógrafos analógicos clásicos tienen anchos de banda de 1-3 octavas y los sismógrafos digitales pueden tener un ancho de banda de 6-12 octavas. Sin embargo, el ancho de banda de la señal de muchas componentes dominantes del ruido sísmico del subsuelo puede ser menor que 1 octava. En consecuencia, la frecuencia de las mediciones es realmente el rango de frecuencias. Sin embargo, por razones prácticas, será usada la frecuencia promedio para representar la medición.

Para la frecuencia promedio, se usa la frecuencia central geométrica $f_{0}$ :

$$
\begin{aligned}
& f_{0}=\sqrt{f_{1} f_{2}}=\sqrt{f_{1} f_{1} 2^{n}}=f_{1} 2^{n / 2}, \\
& f_{1}=f_{0} 2^{-n / 2} \quad \text { у } \quad f_{2}=f_{0} 2^{n / 2} .
\end{aligned}
$$

Para filtros angostos, la frecuencial geométrica central es casi la misma que la frecuencia promedio.

Si se comparan las relaciones con señales en el dominio del tiempo y de la frecuencia, las unidades no tienen importancia; sin embargo, las curvas de Peterson están representadas en aceleración, de modo que la densidad de potencia espectral debe ser expresada en aceleración cuando se calcula a partir de señales en el dominio del tiempo.

La unidad más común para el sismograma original es la velocidad, algunas veces aceleración y raramente desplazamiento. Las relaciones entre las densidades de potencia espectral en cada unidad se han establecido en (3.4) y (3.5). 
Se puede hacer algún ejemplo:

Sea una amplitud pico de $13 \mathrm{~nm}$ para la banda de frecuencias $0.7-1.4 \mathrm{~Hz}$, que resulta expresado en $P_{d}$ :

$$
P_{d}=\left(13.10^{-9} / 1.25\right)^{2} /(1.4-0.7)=1.55 .10^{-16} \mathrm{~m}^{2} / \mathrm{Hz} .
$$

La frecuencia central es $\sqrt{0.7 .1 .4}=1.0 \mathrm{~Hz}$ y la densidad de potencia espectral en aceleración es:

$P_{a}=1.55 \cdot 10^{-16} \cdot 16 \pi^{4} 1^{4}=2 \cdot 4 \cdot 10^{-13}\left(\mathrm{~m} / \mathrm{s}^{2}\right)^{2} / \mathrm{Hz}$ ó

$-126 \mathrm{~dB}$ relativos a $1\left(\mathrm{~m} / \mathrm{s}^{2}\right)^{2} / \mathrm{Hz}$.

El nivel $-126 \mathrm{~dB}$ pareciera alto comparado con el nivel espectral que es alrededor de $-136 \mathrm{~dB}$, fig, 3.1. ¿Cómo se puede explicar esto?.

Se debe recordar que la fórmula está basada en que la amplitud es el promedio de la amplitud pico, mientras que lo que se ha usado es la mayor amplitud en la ventana. Si la amplitud pico promedio es 3 veces menor que la amplitud máxima, el nivel debiera ser corregido por $-10 \log \left(3^{2}\right)=-9.5 d B$ y las medidas en ambos dominios serían similares.

Hay una regla de oro que es la siguiente: Un desplazamiento pico de $1 \mathrm{~nm}$ en $1 \mathrm{~Hz}$ significa un buen lugar en términos de ruido ambiente.

Un punto para ser considerado es que, en realidad, el ruido es de naturaleza aleatoria, (cualquier perturbación "predecible" no es ruido como se define aquí). La potencia espectral estimada por la simple transformada de Fourier para una ventana de tiempo de una muestra de datos crudos, es también aleatoria. El error standard de tal estimación es muy alto. Realmente, cuando se expresa el nivel de ruido como densidad de potencia espectral o RMS en una determinada banda de frecuencias, se está asumiendo implícitamente, como ya se ha mencionado, que el ruido es un proceso estacionario, que significa que sus características estadísticas no son tiempodependientes 0 al menos varían suficientemente lento para ser consideradas constantes en un cierto intervalo de tiempo.

De manera que, si se necesita una estimación real del nivel de ruido en un lugar dado, se debe realizar una estimación del promedio de la densidad de potencia en varias muestras de ventanas-tiempo sobrepuestas o algún suavizado espectral para decrecer la varianza estimada. 


\section{Capítulo 4 \\ ESTACION SISMOLÓGICA LPAD \\ LA PLATA DIGITAL}

\subsection{Antecedentes}

\subsubsection{Reseña histórica}

La primera estación sismológica instalada en el Observatorio Astronómico de La Plata data del año 1907, durante la dirección de Francisco Porro di Somenzi, cuando se comenzó a registrar con un sismógrafo mecánico Vicentini de tres componentes, las dos horizontales con un período propio de 2 seg y la vertical con un período propio de $1 \mathrm{seg}$.

Durante la posterior dirección de William J. Hussey se adquirió, en 1912, el equipo de sismógrafos mecánicos Mainka, de dos componentes horizontales de 450 $\mathrm{kg}$ de masa cada una, con un período propio de $8 \mathrm{seg}$, una razón de amortiguamiento 4:1 y una amplificación de 150. Estos sismómetros funcionaron en forma ininterrumpida hasta el año 1991.

Johannes Hartmann (Director durante el período 1921-1934), logró transformar la estación sismográfica platense, casi insensible en esa época, en una estación sumamente útil para la especialidad. Dentro de otros resultados, logró mejorar el equipo existente proveyéndolo de un aparato Wiechert para el registro de la componente vertical, con un período propio de $3 \mathrm{seg}$ y amplificación de 80 . De esta manera se reemplazó al sismógrafo Vicentini que tenía muchos defectos tales como período muy pequeño, roce muy grande, carecía de amortiguador y de apropiado registro de marcas de tiempo. Este sismómetro funcionó hasta el año 1957.

El director del Servicio Sismológico, entre los años 1907 y 1922, fue el Dr. Galdino Negri, para el período entre 1922 y 1925 fue el Dr. W.J. Hussey y a partir de 1925 hasta el año 1935 la conducción del mismo le fue confiada al Dr. Lunkenheimer, quien se ocupó de dar a publicidad los datos obtenidos tanto en los boletines mensuales provisorios, como en la publicación titulada Resultados Sismométricos, iniciada por Hartmann.

Durante la dirección del Servicio Sismológico del Ing. Simón Gershanik (19351978) y con la intención de incrementar la sensibilidad de la estación, fue adquirido un equipo de sismógrafos electromagnéticos Sprengnether de dos unidades, de largo período (período propio de $14 \mathrm{seg}$ ) para el registro de los movimientos horizontales, 
que comenzó a funcionar en forma regular a partir de 1951 y hasta el año 1957, duplicando el número de terremotos que solían registrarse anualmente con el instrumental mecánico.

\subsubsection{Estación sismológica LPA.}

Con el auspicio de la Unión Geodésica y Geofísica Internacional, el Departamento de Defensa de Estados Unidos resolvió hacia 1960 organizar una red de 125 estaciones sismográficas distribuidas en todo el globo, dotadas con equipos homogéneos, constituidos por un juego de sismógrafos electromagnéticos de tres componentes de largo período y otro de igual clase de corto período, provistos de marcas de tiempo y comando a reloj de cuarzo. Esta red fue designada con el nombre de World-Wide Standard Seismographic Net (WWSSN), y su establecimiento formó parte de un proyecto conocido por la sigla VELA.

En atención a sus antecedentes y experiencia en la materia, se le ofreció al Observatorio de La Plata su incorporación a la red. Aceptada la misma, se ubicó en él una de las estaciones de la red planeada, en el pabellón en el cual ya habían funcionado el sismógrafo Vicentini y el Wiechert vertical.

La estación sismológica La Plata (LPA) comenzó a operar el 23 de marzo de 1962 como integrante de la red sismológica mundial. La información sismológica obtenida codificada es enviada, desde entonces, al National Earthquake Information Center, World Data Center A for Seismology, EEUU, y al International Seismological Centre, Gran Bretaña, como contribución en la confección de catálogos globales.

\subsubsection{Instalación}

\section{Ubicación geográfica}

El recinto que alberga los sensores se encuentra en el predio de la Facultad de Ciencias Astronómicas y Geofísicas de la UNLP (FCAGLP). Sus coordenadas geográficas son Latitud: $34^{\circ} 54^{\prime} 32^{\prime \prime} \mathrm{S}$, Longitud: $57^{\circ} 55^{\prime} 55^{\prime \prime} \mathrm{W}$, Altitud sobre el nivel del mar: $15 \mathrm{~m}$.

La Facultad se encuentra en el cuadrante noreste de la ciudad de La Plata, dentro del bosque platense, aproximadamente a $2 \mathrm{~km}$ del centro comercial y de la estación de ferrocarril ; con un polo petroquímico a pocos $\mathrm{km}$ y con dos estadios de fútbol a centenas de metros (Fig. 4.1).

La ciudad de La Plata está aproximadamente a $6.4 \mathrm{~km}$ al sudoeste del Río de La Plata, un estuario a $160 \mathrm{~km}$ tierra adentro del Océano Atlántico y $60 \mathrm{~km}$ al sudeste 
de la ciudad de Buenos Aires. La cadena montañosa más cercana, Sierras Pampeanas, está aproximadamente a $700 \mathrm{~km}$ al oeste-noroeste.

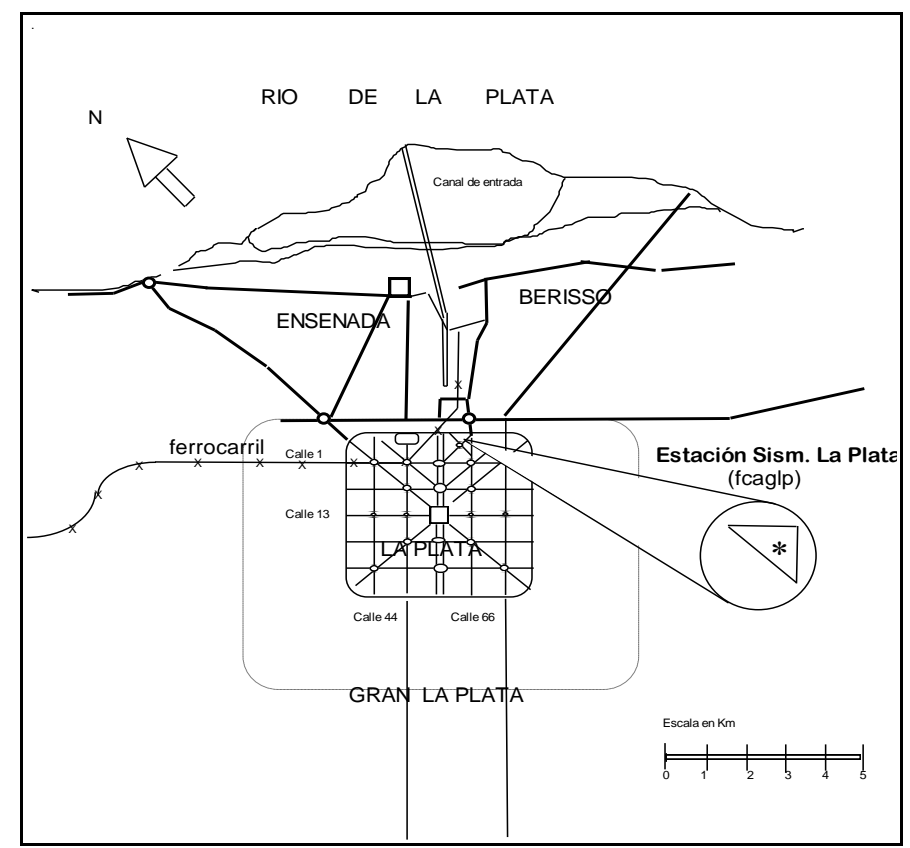

Figura 4.1: Mapa de ubicación de la estación sismológica LPA. (Pinciroli y otros, 1997).

\section{Relación de la estación con otras construcciones}

En el predio hay dos cúpulas de $2.4 \mathrm{~m}$ de diámetro y $2.7 \mathrm{~m}$ de altura. Una está aproximadamente a $60 \mathrm{~m}$ al oeste del recinto y la otra a $90 \mathrm{~m}$ hacia el sudoeste. Hay una torre de $38 \mathrm{~m}$ de altura que alberga sensores meteorológicos y antenas de comunicación aproximadamente a 180m hacia el noroeste del recinto.

\section{Geología local}

El área en que está ubicada es una planicie aluvional de tipo costero de un radio de $400 \mathrm{~km}$. Está compuesta por loess cuaternario con un espesor de aproximadamente 466m (L.E.M.I.T., Laboratorio de entrenamiento multidisciplinario para la investigación tecnológica). Su conformación está dada por:

Formación Ensenadense: desde el nivel del suelo hasta los 28 metros de profundidad.

Formación Araucano-Puelchense: desde los 28 a los 43 metros. 
Formación Mesopotámico superior "verde": desde los 43 a los 278 metros.

Formación Mesopotámico inferior "rojo": desde los 278 hasta los 466 metros.

Basamento cristalino (Precámbrico): desde los 466 metros en adelante.

\section{Características del recinto}

Los pilares: se encuentran en el piso del recinto de los sismómetros, en un subsuelo a $5 \mathrm{~m}$ de profundidad. Tienen una penetración de $1 \mathrm{~m}$ por debajo del nivel del suelo y están fijados en material poco consolidado ya que la roca sólida se encuentra a una profundidad de $460 \mathrm{~m}$. Los dos pilares, uno para corto y otro para largo período, están construidos de ladrillo, concreto, reforzados con hierro y separados del piso del sótano.

Las condiciones ambientales: en los recintos de los sensores y de registración hay deshumidificadores y estufas, de manera de no tener grandes variaciones térmicas o de humedad, estacionales y/o diarias.

\subsubsection{Instrumental}

La estación sismológica LPA, desde el año 1962 y hasta el presente cuenta con sismógrafos Benioff (United Electro Dynamics, EEUU), tres componentes en corto período con período propio de $1 \mathrm{seg}$, y en largo período con sismógrafos Sprengnether (Sprengnether, EEUU), tres componentes en largo período, con período propio de 15 seg, dos componentes horizontales y una vertical en cada caso.

Los registros, que son diarios, se obtuvieron en papel fotográfico, en corto período con galvanómetro de $\mathrm{T}=0.75 \mathrm{seg}$ y amplificación de 3125 y en largo período con galvanómetro de $\mathrm{T}=100 \mathrm{seg}$ y amplificación 750 , hasta septiembre de 1987. A partir del mismo año se cambió a papel termosensible, registrándose, sólo en las tres componentes de largo período (con amplificación 375) y en la vertical de corto período con amplificadores Kinemetrics, para obtener registros en forma analógica.

\section{2 - Estación sismológica LPAD}

\subsubsection{Características Generales}

Habida cuenta que a partir de los años 90 se desarrollaron redes globales de sismógrafos digitales, que los registros obtenidos con este tipo de instrumental son aptos para la aplicación de programas para el tratamiento de señales y que en la 
Sismología se comenzaron a desarrollar programas específicos, se vio la necesidad de modernizar la estación sismológica LPA.

En tal sentido, a partir de agosto de 1994 se obtuvieron en esta estación, además de los sismogramas analógicos en papel termosensible, los primeros sismogramas digitales en el marco del Proyecto "Sismología Digital en el Observatorio" de la UNLP. Se usaron los mismos sismómetros de largo período de la red internacional WWSSN con masa inercial de aproximadamente $10 \mathrm{~kg}, \mathrm{y}$ frecuencia natural en $0.067 \mathrm{~Hz}$ (15 segundos de período).

\subsubsection{Primer etapa (LPAD1)}

A fin de obtener registros digitales, fue necesaria la modificación de la interfase para la adquisición de datos. En una primera etapa, se diseñó y construyó un sistema con los amplificadores y filtros necesarios para lograr una respuesta plana al desplazamiento, en banda ensanchada (Fig. 4.2) (Sabbione y otros, 1995).

El sistema producía los niveles suficientes de señal a efectos de excitar la placa de conversión analógica digital (Data Translation) instalada en una PC dedicada a la adquisición de datos. Esta placa permitía realizar la conversión analógica digital en 12 bits, admitiendo hasta 16 entradas con cuatro pasos de ganancia x1; x2; x4 y x8, con un rango de frecuencias de muestreo posibles entre 10 y $120 \mathrm{~Hz}$.

El proceso de adquisición de datos sismológicos se efectuaba con el programa Mdetect desarrollado por IASPEI (International Association of Seismology and Physics of the Earth Interior). Este software controlaba la detección de eventos mediante el algoritmo desarrollado por Tottingham y Lee y procesaba las señales en tiempo real (Lee, 1989); la información seleccionada se grababa en el disco rígido de la PC.

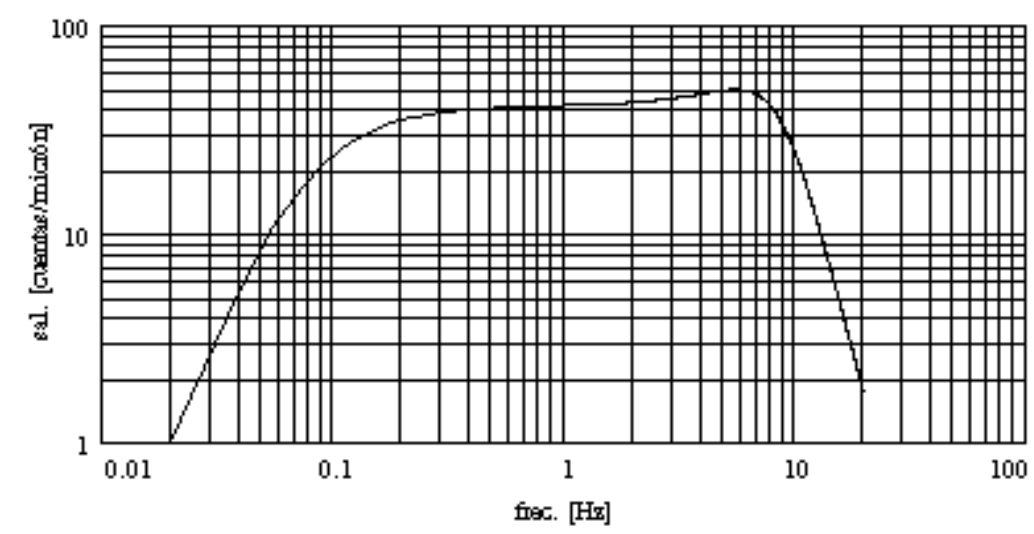

Figura 4.2: Curva de transferencia inicial de LPAD (Sabbione y otros, 1995). 
Esta curva puede ser también expresada en función de sus polos y ceros (ver 2.1) en formato GSE (Group pf Scientific Experts) por ejemplo:

Estación LPAD1 (desplazamiento):

CAL1 BALPA PAZ

7

$-0.4210 \quad 0.0$

$-0.4210 \quad 0.0$

$-0.4210 \quad 0.0$

$-30.000 \quad 46.15$

$-30.000-46.15$

$-30.000 \quad 46.15$

$-30.000-46.15$

3

$0.0 \quad 0.0$

$0.0 \quad 0.0$

$0.0 \quad 0.0$

3.333E06

donde se indica que posee 7 polos, tres de los cuales son reales y 3 ceros, indicando la última línea el factor de escala para normalizar a desplazamiento del suelo en $\mathrm{nm}$.

En una primera etapa se trabajaba en cuatro canales, tres para los sensores y uno para las marcas de tiempo. Se adoptó una frecuencia de muestreo de $20 \mathrm{~Hz}$, con 10 minutos de tiempo de pre-evento y 30 minutos de post-evento. Con dichos parámetros se generaban archivos de aproximadamente 380 Kbytes.

\subsubsection{Segunda etapa (LPAD2)}

En una segunda etapa, viendo la conveniencia de contar con registros en velocidad, se realizaron los ajustes de los parámetros de registro por evento y se diseñó y construyó un amplificador especial, con ancho de banda controlado, para habilitar el "disparo por canal filtrado", que produjo una notable simplificación en la tarea de recuperación de datos.

A partir de junio de 1996 la estación digital LPAD2 quedó conformada por sensores en tres componentes de la red VELA, tarjeta de adquisición Data Translation/IASPEI registrando en velocidad con CAD de 12 bits, a $20 \mathrm{mps}$ con sensibilidad:50 ctas/um/s que se cambió a 15 ctas/um/s en diciembre de 1996 (Fig. 4.3).

Los polos resultaron ser:

$P 1,2=-0.295+/-j 0.301$ y p3,4,5,6 =-30.303+/-j45.56 (1/s) 


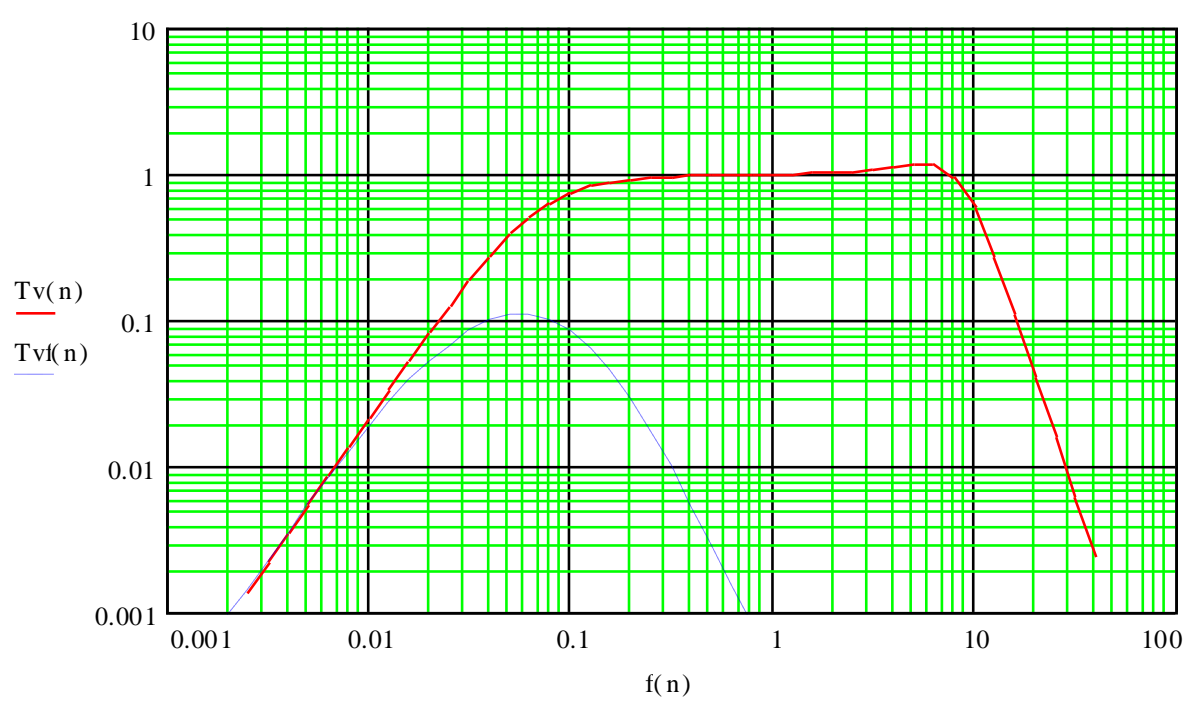

Figura 4.3 Curva de ganancia normalizada de LPAD2 y curva de filtro de disparo

\subsubsection{Tercer etapa (LPAD3)}

A partir del 18 de octubre de 2002 se ha reemplazado la tarjeta de adquisición IASPEI por un adquisidor de datos AD7710X3, conversor analógico-digital, que contiene 3 conversores de 16/19 bits de ANALOG DEVICES AD7710, cuyas salidas van a un procesador PIC16C54, donde se hace el muestreo a $25 \mathrm{mps}$, el multiplexado de los canales y el filtrado antialias. Los CAD poseen un filtro digital con respuesta $(\sin x / x)^{3}$. La ganancia de los conversores es variable de 1 a 128 , en pasos combinables por multiplicación. Luego se ha instalado un conversor RS232 y la salida a la PC, conectados a una alimentación de $12 \mathrm{~V}$ para evitar la suspensión de la registración frente a eventuales cortes en el suministro de energía.

Sistema de adquisición: Se utiliza el programa AD7710H.exe, el cual identifica el procesador que maneja la estación. Al entrar a dicho programa comienza automáticamente la registración. Durante la operación normal se muestra en pantalla en una ventana de un minuto de duración, la evolución de la señal en tiempo real, permitiendo ver una de las componentes. El registro es continuo y se graba automáticamente en archivos de una hora de duración, resultando en binario, a 16 bits, de un tamaño de 540K. El sistema de tiempo utilizado es el que corresponde a la estación analógica.

Visualización y extracción de eventos: El programa BANDAS (Departamento de Volcanología, Museo Nacional de Ciencias naturales, CSIC, Madrid) permite hacer un preprocesamiento simple de los datos para extraer de los registros de una hora, 
aquellos intervalos que contengan información útil. Se generan archivos con formato ascii para cada una de las tres componentes (identificados a través de: día juliano, hora y minuto de la primera muestra extractada) más un archivo de texto con la siguiente información: número de muestras, frecuencia de muestreo, frecuencia de corte del filtro antialias ( $6 \mathrm{~Hz}$ ), fecha, nombre de la estación, número de canales y componentes asociadas a los mismos, ganancia y sensibilidad.

\section{3- Uso de lo estación.}

El software utilizado inicialmente para realizar el procesamiento fue el PITSA (Programmable Interactive Toolbox for Seismological Análisis, versión 4.0, Scherbaum et al., 1993), que ofrece un número de herramientas para manipular las trazas sísmicas tales como picado interactivo y automático de fases, diagramas de movimiento de partícula (en dos o tres dimensiones), estimación de la magnitud, rotación de componentes, aplicación de diferentes filtros tales como Butterworth pasa banda, pasa bajos, pasa altos, de polarización, gaussiano, corrección por la función transferencia del instrumento, cálculo de FFT (fast Fourier transform) y FFT inversa, etc.. Esto hizo que el PITSA fuera una herramienta muy versátil para el procesamiento de señales digitales.

A modo de ejemplo, del conjunto de terremotos registrados en forma digital en la Estación Sismológica La Plata a partir de agosto de 1994 se han seleccionado dos (Fig. 4.5, gráficos 1 a 4), cuyos parámetros focales se presentan en la tabla 4.1.

\begin{tabular}{|c|c|c|c|c|c|c|c|l|}
\hline Fecha & Latitud & Longitud & $\begin{array}{c}\text { Hora } \\
\text { origen }\end{array}$ & $\begin{array}{c}\text { Prof. } \\
\text { Km }\end{array}$ & Mag. & $\begin{array}{c}\text { Dist. a } \\
\text { LPA }\end{array}$ & $\begin{array}{l}\text { Back } \\
\text { Azim }\end{array}$ & $\begin{array}{l}\text { Ubicación } \\
\text { Geográfica }\end{array}$ \\
\hline $19 / 08 / 94$ & $26^{\circ} .64 \mathrm{~S}$ & $63^{\circ} .42 \mathrm{~W}$ & $10: 02: 51.8$ & 564 & 6.4 & $1055 \mathrm{~km}$ & $329^{\circ}$ & $\begin{array}{l}\text { Sgo. del } \\
\text { Estero }\end{array}$ \\
\hline $02 / 06 / 95$ & $31^{\circ} .83 \mathrm{~S}$ & $71^{\circ} .48 \mathrm{~W}$ & $19: 07: 22.5$ & 70 & 5.4 & $1303 \mathrm{~km}$ & $281^{\circ}$ & Chile Central \\
\hline
\end{tabular}

Tabla 4.1: Parámetros focales de los eventos seleccionados procesados con PITSA 


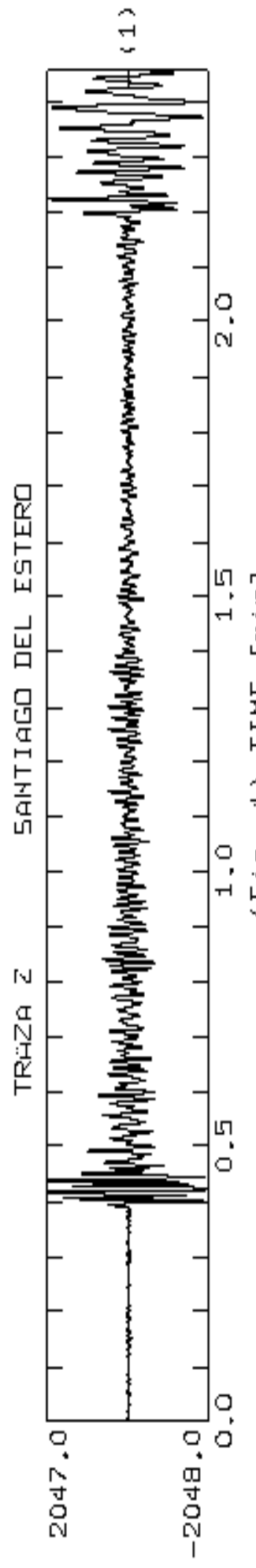

[5equenj] - Zd5

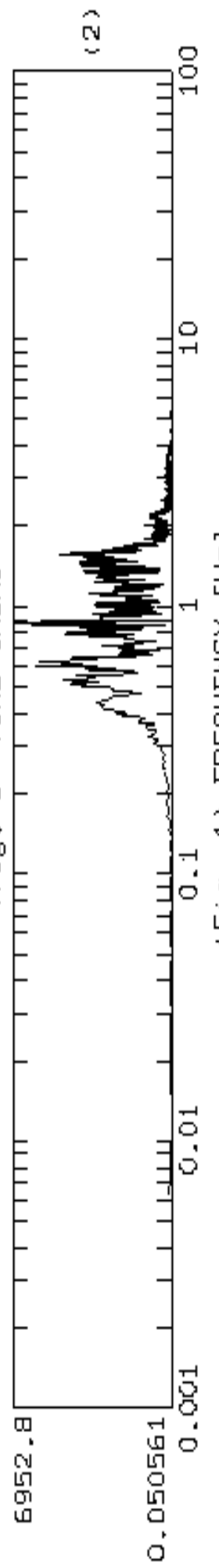

0ㄴㅣㅓㄷㅗ

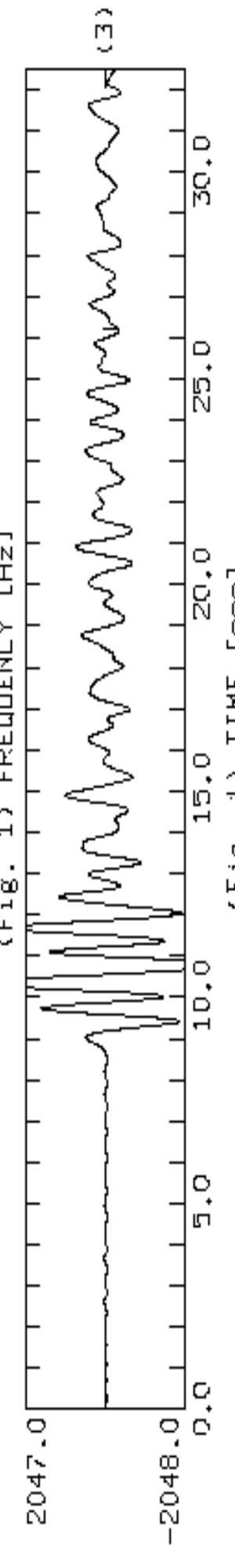

$d-\mathrm{MOOZ}$

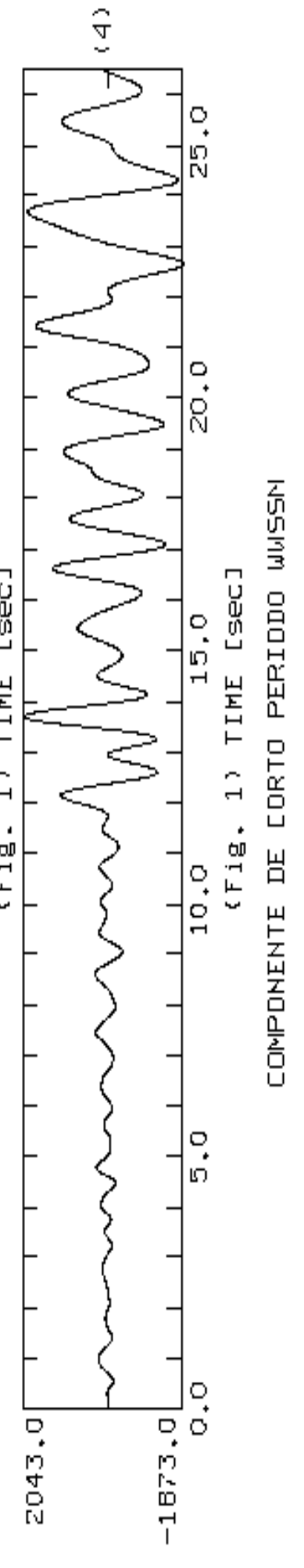

$5-\operatorname{Mol} 2$ 


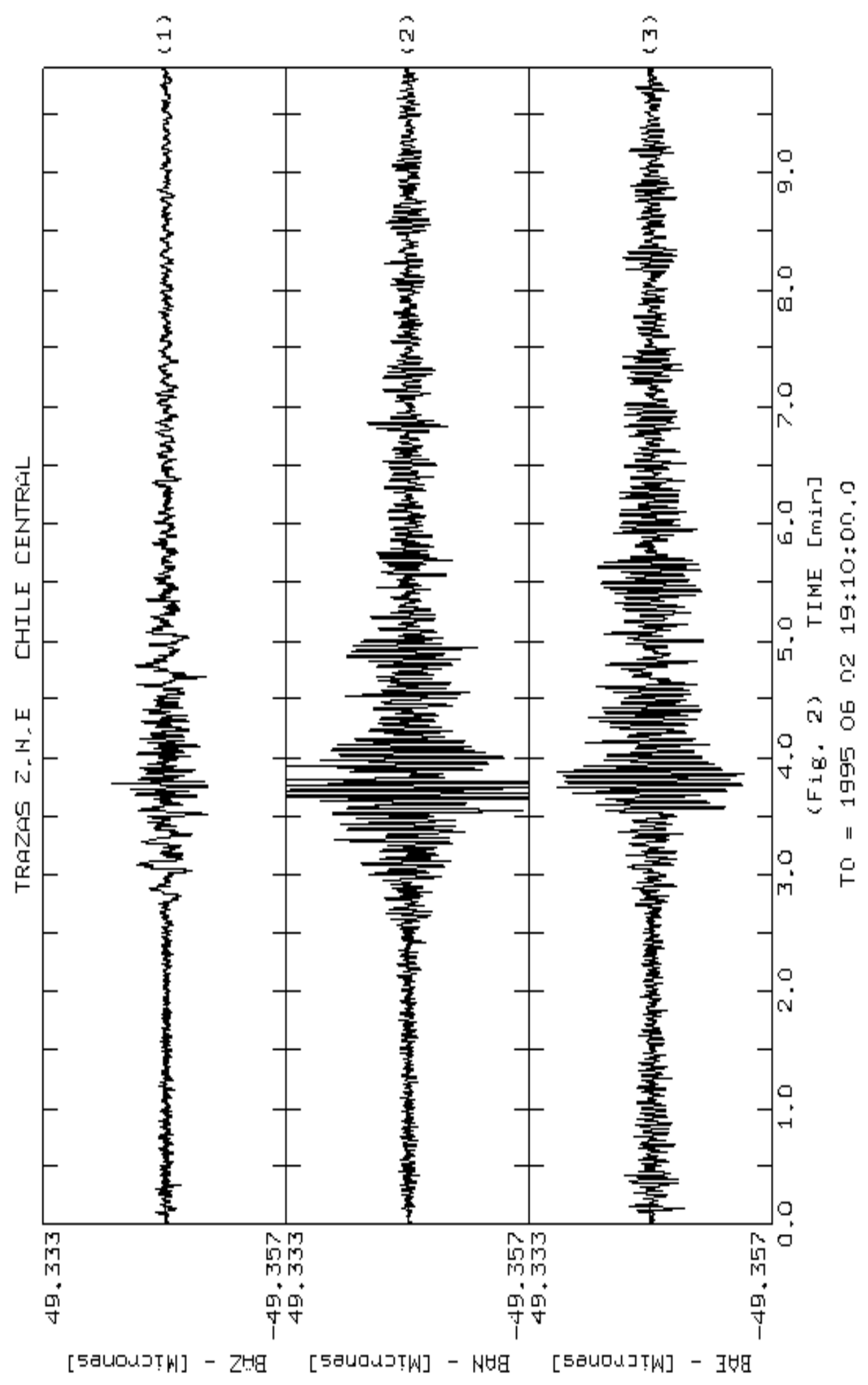




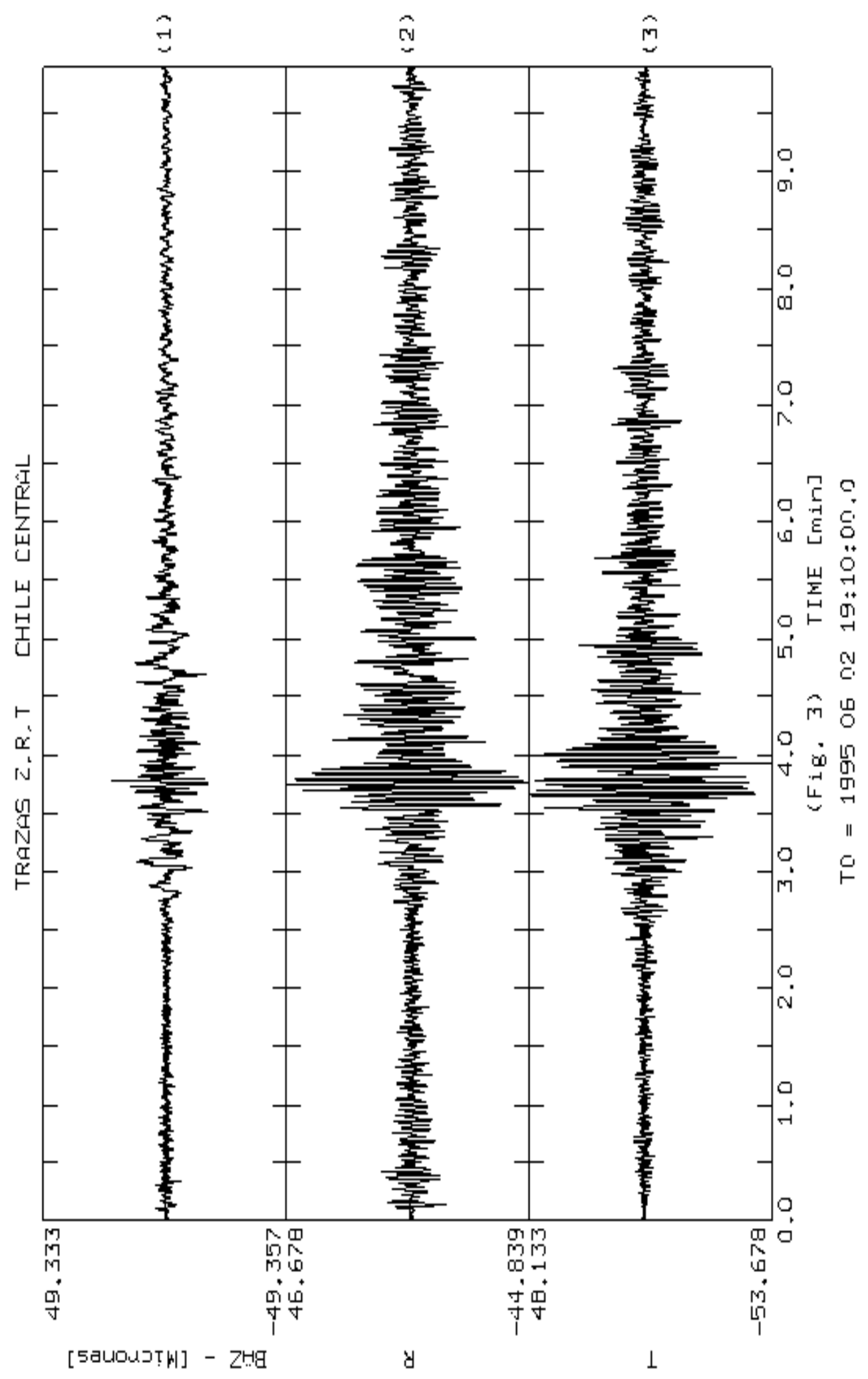




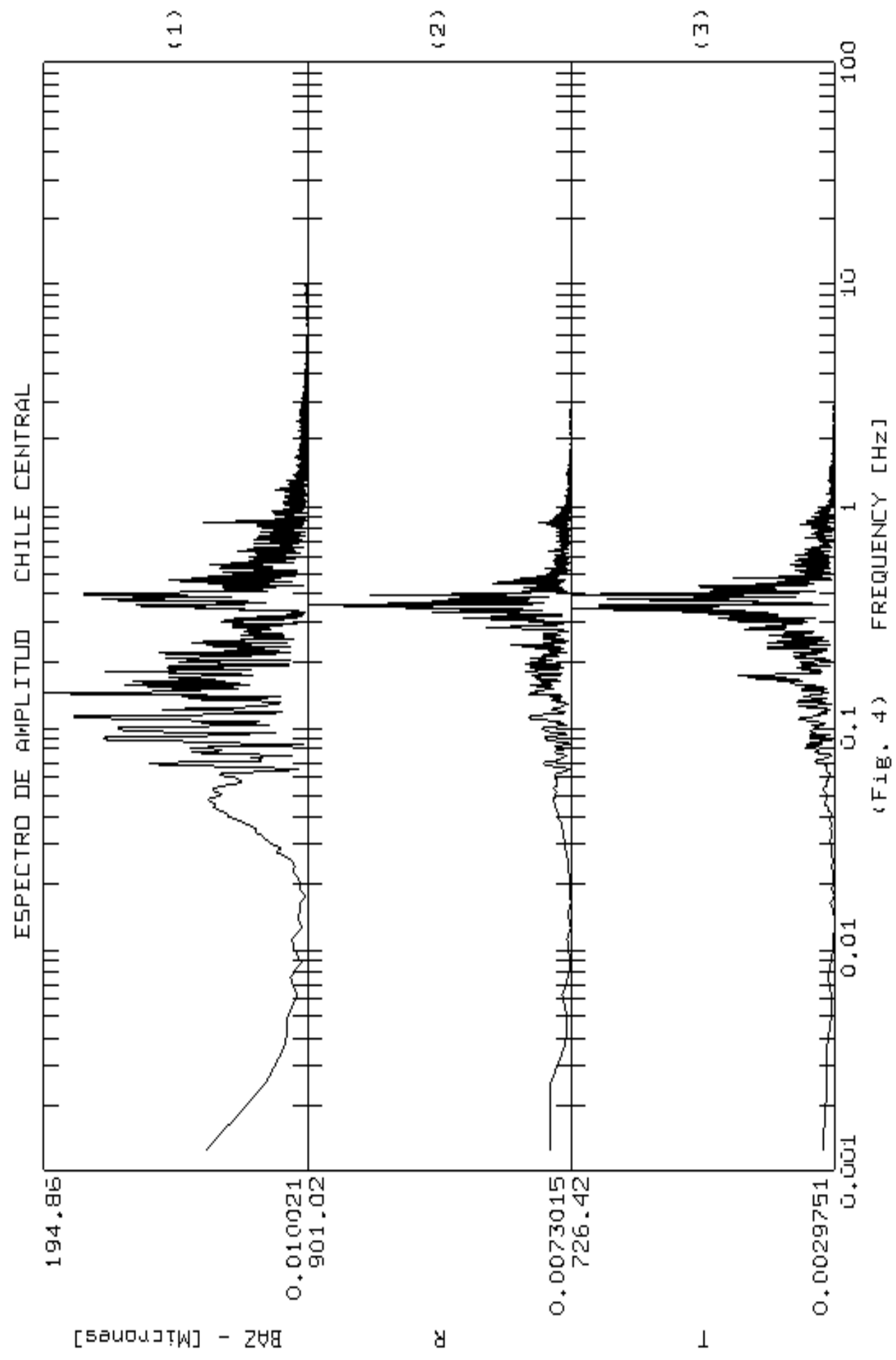

Figura 4.5: Eventos procesados con el software PITSA, 4 gráficos (Sabbione y otros, 1995). 
El evento del 19/08/94 (Fig. 4.5-1) se obtuvo en forma digital conservando las características originales del corto período de la red WWSSN. En dicha figura se puede observar la traza original de la componente vertical, luego se obtuvo el espectro de amplitud de dicha traza y a efectos de poder observar con mayor claridad el comienzo de las fases $\mathrm{P}$ y $\mathrm{S}$ se realizó un zoom de dos porciones de la traza original.

En la figura 4.5-2 se pueden observar las trazas originales de las tres componentes, en las que se ha expresado en el eje vertical el desplazamiento del suelo (en micrones) en la estación LPA durante la ocurrencia del terremoto. En la figura 4.5-3 se ha representado la rotación de las componentes NS y EW en componentes Radial y Tangencial lo que permite que las fases longitudinales y transversales queden mejor evidenciadas. En la figura 4.5-4 de esas mismas tres componentes se ha graficado el espectro de amplitud.

Con posterioridad al PITSA, se utilizó el software SAC (Seismic Análisis Code, Tapley 2000). A modo de ejemplo se han seleccionado dos eventos (Tabla 4.2) registrados en la estación sismológica LPAD2 (La Plata digital, LPAdig) y PDAS (La Plata, sensores BB13 (ver 4.4.1)), Figs. 4.6 y 4.7.

\begin{tabular}{|c|c|c|c|c|c|c|}
\hline Fecha & Lat. $\left(^{\circ}\right)$ & Long. $\left(^{\circ}\right)$ & Prof. $(\mathrm{km})$ & Mag. $(\mathrm{mb})$ & Dist.a LPA & Lugar \\
\hline $02 / 09 / 97$ & $3.887 \mathrm{~N}$ & $75.740 \mathrm{~W}$ & 222 & 6.4 & $42^{\circ} 07$ & Colombia \\
\hline $04 / 09 / 97$ & $26.535 \mathrm{~S}$ & $178.322 \mathrm{E}$ & 618 & 6.3 & $98^{\circ} 97$ & Sur Is. Fiji \\
\hline
\end{tabular}

Tabla 4.2: Parámetros focales de los eventos seleccionados procesados con SAC

Todos los sismogramas considerados fueron expresados en función de las velocidades (en el caso del PDAS las trazas debieron integrarse a tal efecto), además fueron filtrados con un filtro pasa altos, con frecuencia de corte en $0.025 \mathrm{~Hz}$. 


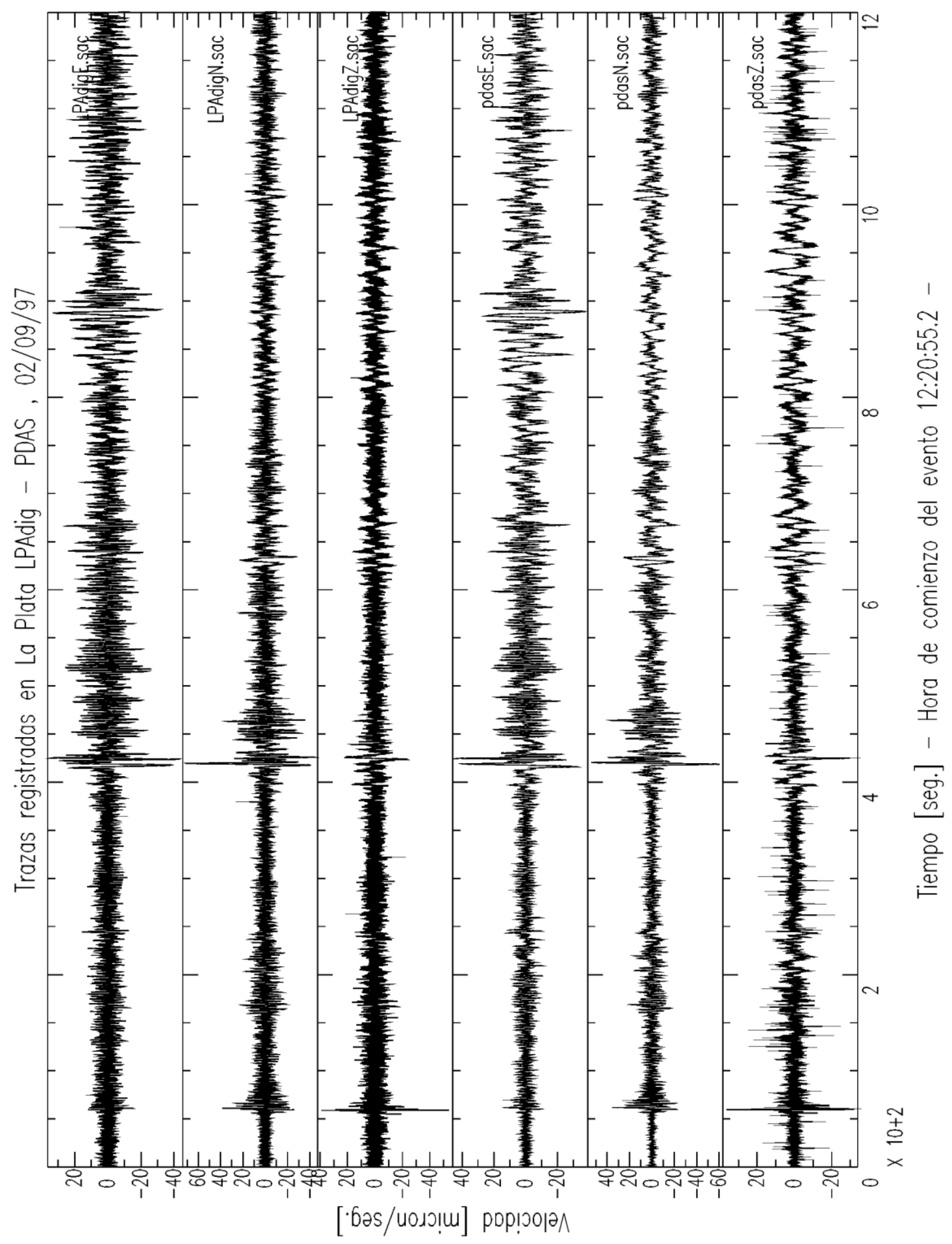

Figura 4.6: Trazas correspondientes al sismo de Colombia registrado en La Plata. (de Sabbione y otros, 1998) 


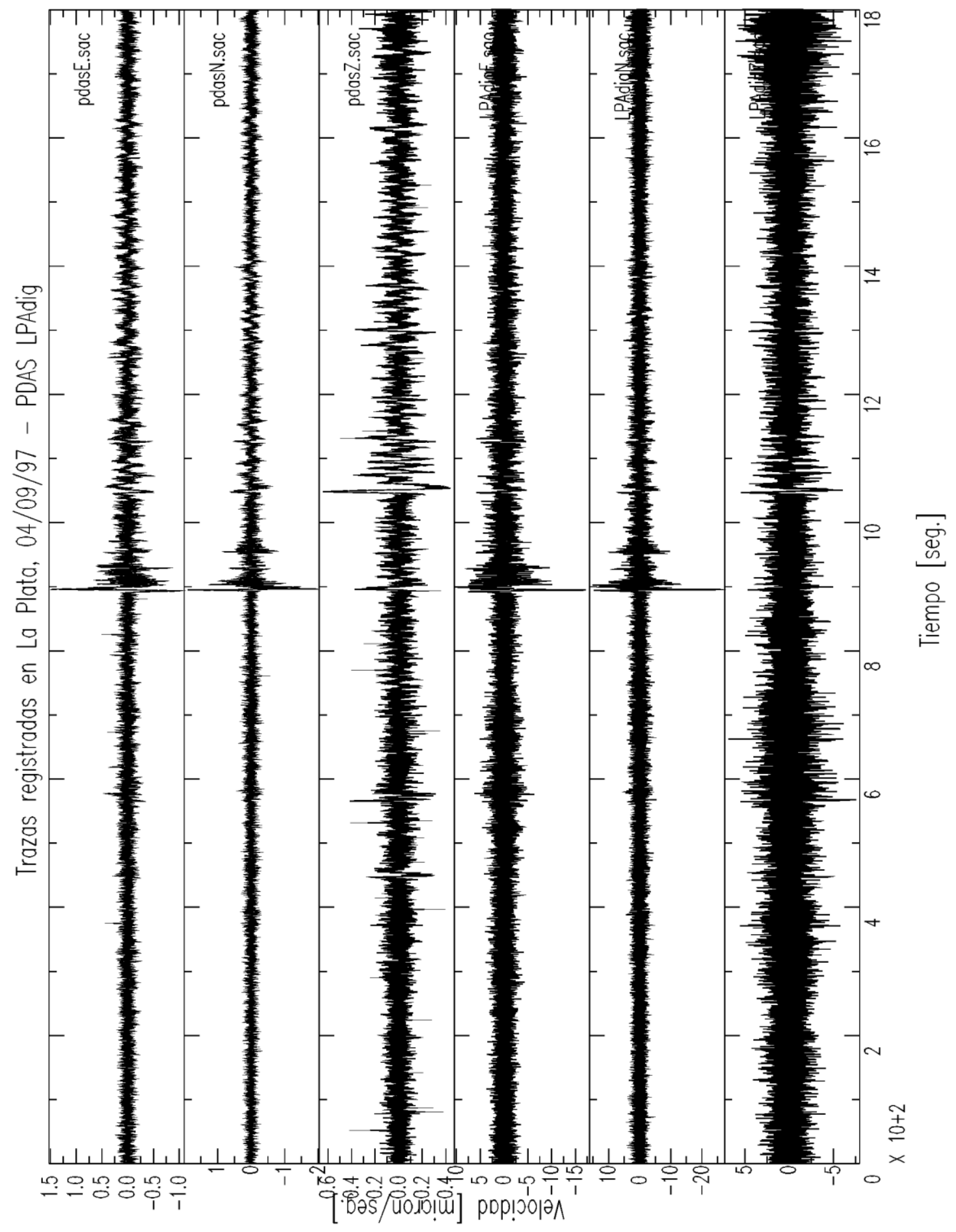

Figura 4.7: Trazas correspondientes al sismo de Islas Fiji registrado en La Plata. (de Sabbione y otros, 1998) 


\section{4- Ruido sísmico en la estación}

Las señales sísmicas siempre contienen ruido y es importante saber tanto la fuente del ruido como saber medirlo, El ruido puede tener dos orígenes: ruido generado en el instrumental y ruido sísmico "real" debido a vibraciones de la Tierra (ver 1.4). Normalmente, el ruido instrumental está debajo del ruido sísmico aunque muchos sensores pueden tener una banda de frecuencia donde el ruido instrumental es dominante (por ejemplo un acelerómetro a bajas frecuencias).

Todos los sismogramas muestran alguna clase de ruido cuando la ganancia se aumenta y en la mayoría de los lugares del mundo, se observa ruido de aspecto armónico (llamado microsismo) en la banda de 0.1-1.0 Hz, excepto que estén oscurecidos por un alto nivel de ruido local.

Obviamente el nivel de ruido en una estación debe ser especificado en frecuencias diferentes.

Intuitivamente, el modo más simple es medir los movimientos de la Tierra en diferentes bandas de frecuencia y graficar la amplitud como una función de la frecuencia o el período. Este fue de hecho el modo en que se hizo en una primera etapa en la estación con amplitudes de aceleración.

\subsubsection{Aspectos del ruido sísmico en aceleración}

Durante el año 1997 (Pinciroli y otros, 1997), se realizaron mediciones del ruido en aceleración en una banda comprendida entre los $0.05 \mathrm{~Hz}(\mathrm{~T}=20 \mathrm{~s})$ y los $16 \mathrm{~Hz}$ ( $\mathrm{T}=0.06 \mathrm{~s})$, con el objeto de conocer su nivel y distribución espectral, utilizando un acelerómetro digital de banda ancha de tres componentes, cedido en calidad de préstamo de uso al Depto. de Sismología, a través del Convenio existente entre el Instituto Antártico Argentino, el Programma Nazionale di Ricerche in Antartide y la Universidad Nacional de La Plata.

El instrumental utilizado consiste en tres acelerómetros de banda ancha BB13 Teledyne Geotech realimentados por fuerza, de operación vertical u horizontal, con una masa de $5 \mathrm{~kg}$, con ancho de banda de CC a $20 \mathrm{~Hz}$ y sensibilidad de $102 \mathrm{~V} / \mathrm{m} / \mathrm{s}^{2}$; un sistema de adquisición de datos portátil PDAS100 de Teledyne Geotech con conversor A/D de 16 bits (ganancia ajustable o fija), procesador digital de señales (DSP), 3 canales de entrada para sensores y 3 auxiliares, memoria de estado sólido CMOS de $9 \mathrm{Mb}$, reloj incorporado con cristal compensado térmicamente (TCXO) y un software de adquisición con 13 frecuencias de muestreo entre 0.1 y 1000 mps, 4 
formas de disparo por evento, filtro antialias automático con la frecuencia de muestreo de 0.4 veces.

La recolección de los datos se realizó en una frecuencia de muestreo de 50 mps con lo cual el equipo aplica automáticamente un filtro antialias con corte en los 20 $\mathrm{Hz}$, con una atenuación de $120 \mathrm{db}$ en $25 \mathrm{~Hz}$ (frecuencia de Nyquist), por lo que se consideraron válidos los datos hasta $16 \mathrm{~Hz}$. Durante la adquisición se aplicó la opción de un filtro pasa altos de un polo, con una frecuencia de corte de $0.01 \mathrm{~Hz}$, para evitar problemas de inestabilidad térmica en los sensores.

En la Fig. 4.8 se presenta la curva de respuesta en amplitud del acelerómetro completo incluyendo todos los filtros mencionados.

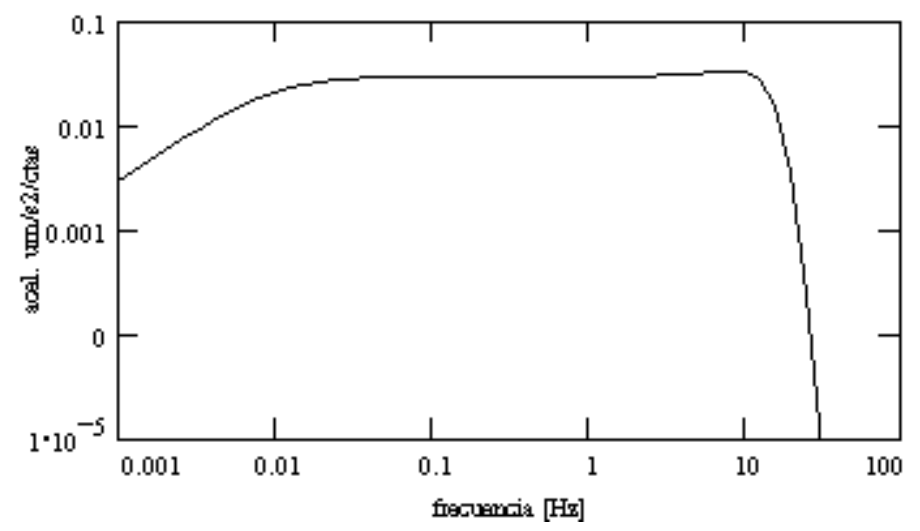

Figura 4.8: Curva de respuesta del acelerómetro PDAS100, BB13 utilizada en la medición (Pinciroli y otros, 1997).

Se programó el equipo para la toma de datos según el siguiente detalle:

- Archivos de 10 minutos cada tres horas, a efectos de poder evaluar el aporte del ruido cultural, producido por la actividad del entorno urbano que rodea a la estación.

- Con el objeto de obtener mayor detalle en las bajas frecuencias, se obtuvieron también archivos de 50 min. a razón de 5 mps.

- Para ciertos eventos particulares tales como viento, actividades deportivas que involucraran gran cantidad de gente, etc., se hicieron disparos manuales del mismo tiempo.

Se descartaron aquellos archivos que pudieran contener información de algún sismo y se estudió especialmente la componente vertical en aceleración, considerando plana la curva de respuesta del instrumento en el rango de interés. Se obtuvieron 4 espectros de otros tantos grupos de 1024 muestras distribuidas uniformemente en los 
600 segundos de datos, aplicando una ventana de Hanning. El promedio de los 4 espectros se suavizó corriendo un promedio móvil de 10 puntos.

Se consideró despreciable el ruido de cuantización y el ruido electrónico del equipo por estar muy por debajo de los valores mínimos medidos en las madrugadas más calmas.

Como herramientas de proceso se usaron el DADISP (DSP Development Corporation, Cambridge, USA): Programa matemático especializado en el procesamiento digital de señales, para PC y el PITSA (Lee, 1989), Versión para PC y para UNIX.

En la Fig. 4.9a se ejemplifica el efecto de un día con tormenta, correspondiendo la curva inferior en el gráfico, a instantes previos al inicio de la misma. Se observa que el viento produce un efecto particular en la estación, aumentando más las amplitudes en bajas frecuencias que en altas.

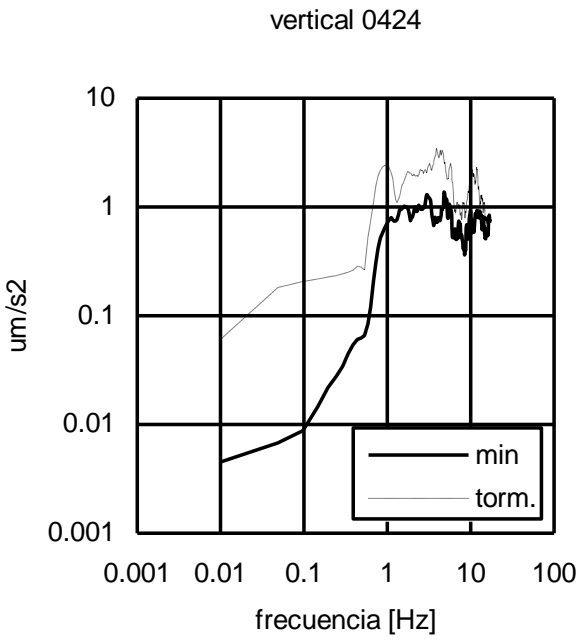

Figura 4.9a: Efecto de una tormenta .
0424 viento (baja frecuencia)

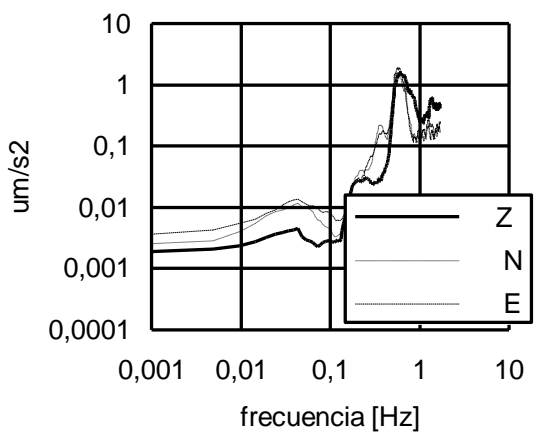

Figura 4.9 b: Detalle en baja frecuencia

En la Fig. 4.9 b se muestra en detalle el espectro de las tres componentes en baja frecuencia, donde queda evidenciado el efecto de la dirección predominante del viento, del cuadrante sur en este caso, que aumenta la amplitud de la aceleración en la componente norte.

En la Fig. 4.10 se caracteriza el ruido cultural encontrado en esta estación sismológica, a través de los espectros correspondientes a una madrugada calma (mínimo) y a una tarde (máximo) en días de semana. 


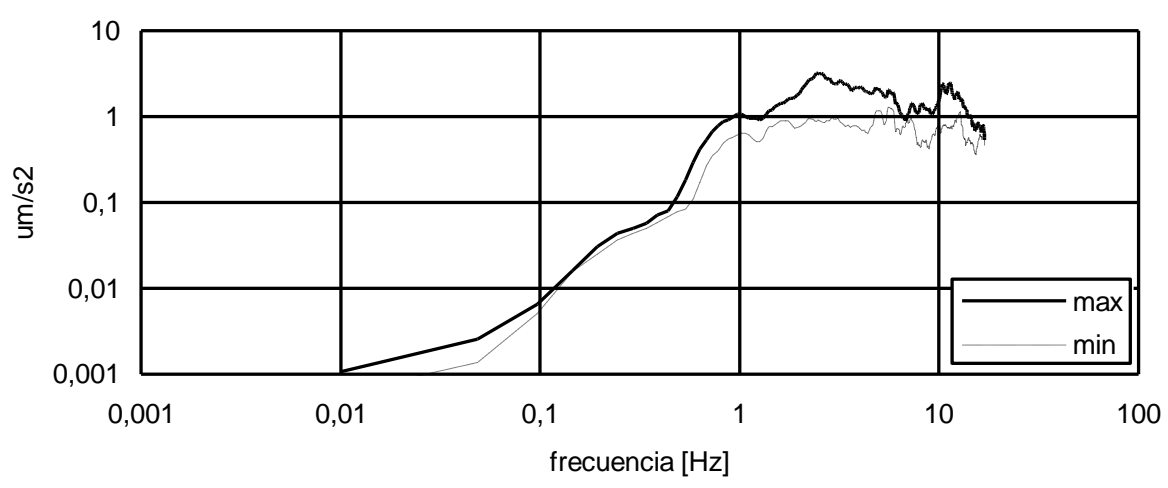

Figura 4.10: Manifestación del ruido cultural en la estación LPA.

De esta figura se desprende que en el espectro del ruido en aceleración, se distinguen claramente dos zonas a partir de $1 \mathrm{~Hz}$, una plana hacia las altas frecuencias y otra con una fuerte caída hacia las bajas.

\subsubsection{Ruido en función del análisis de densidad espectral de potencia en aceleración.}

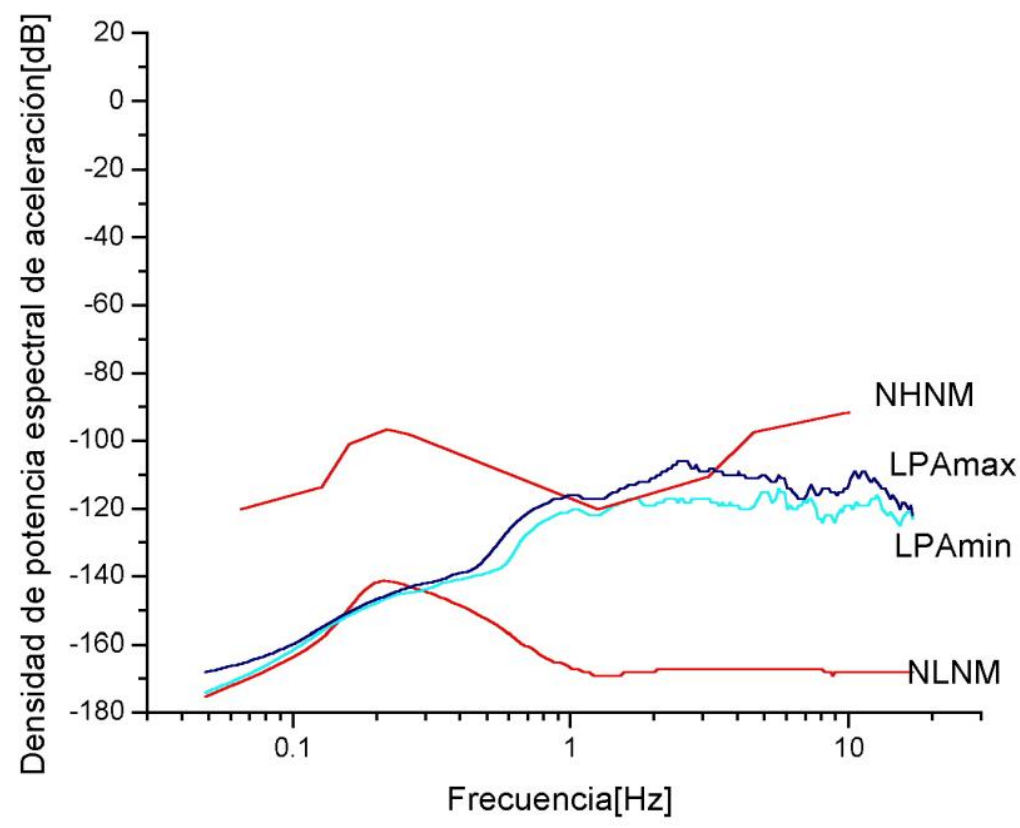

Figura 4.11: Curvas de densidad de potencia espectral de aceleración máxima y mínima y su comparación con las curvas de nivel máximo (NHNM) y mínimo (NLNM) de Peterson (1993), en La Plata 
Teniendo en cuenta lo expresado en el capítulo 3, con respecto a las características del ruido y la conveniencia de representarlo en función de la densidad espectral de potencia, se presenta en la figura 4.11 el ruido en aceleración en la estación sismológica LPA y se lo compara con las curvas de nivel de ruido mínimo y máximo de Peterson (1993).

De la misma se desprende que esta estación tiene una buena relación señalruido para frecuencias por debajo de $1 \mathrm{~Hz}$. Este rango de frecuencias es el que corresponde al caso de sismos regionales y lejanos, que son aquellos que se registran en la estación, ya que la sismicidad local es prácticamente nula (ver cap. 8).

Es claro que la capa de espesor sedimentario tan pronunciada (4.1.3 geología local), es responsable de este fenómeno. 


\section{Capítulo 5 \\ ESTACION SISMOLÓGICA TRWA \\ TRELEW ARGENTINA}

\section{1 - Antecedentes}

\subsubsection{Breve reseña}

Desde julio de 1957 con motivo del Año Geofísico Internacional se instaló un Observatorio Magnético en Trelew, Pcia de Chubut, dependiente del entonces Instituto Superior del Observatorio Astronómico, UNLP. A partir de noviembre de 1970 dicho Observatorio Magnético continuó su registración en un nuevo predio de aproximadamente 44 ha donado por el Instituto Nacional de Tecnología Agropecuaria (INTA).

Las instalaciones han sido usadas, en distintos períodos, para la instalación transitoria de instrumental para mediciones geofísicas como receptores GPS, en varias oportunidades, medidores de ozono y otros; actualmente están funcionando equipos modernos para mediciones geomagnéticas controlados por PC, atendidos por el Depto de Geomagnetismo de la Facultad de Ciencias Astronómicas y Geofísicas.

Desde el año 1997, a partir de una experiencia piloto con una estación sismológica portátil, el Depto. de Sismología ha instalado instrumental en dicho predio. En esta etapa, con un sensor Lennartz de corto período de tres componentes, se realizó el estudio de factibilidad para la instalación de una estación sismológica permanente, a través de una determinación del nivel de ruido en la estación.

El resultado de esta campaña (Rastelli y otros, 1997) permitió, a través del Proyecto "Estación sismológica Trelew, aportes al conocimiento de la litósfera al sur del paralelo $34^{\circ}$ "de la UNLP y un Convenio entre el Osservatorio Geofisico Sperimentale (OGS), Trieste y el Instituto Antártico Argentino, realizar una instalación permanente (Sabbione y otros, 2001).

A partir de abril de 1999 se encuentra funcionando en Trelew una estación digital de tres componentes de banda ancha cedida en calidad de préstamo de uso permanente al Depto. de Sismología por el OGS.

En octubre de 2000, mediante la resolución del Consejo Académico № 107 de la FCAG, el Observatorio Magnético de Trelew se transformó en el Observatorio Geofísico de Trelew, dependiente de la FCAG-UNLP, contando con Normas de funcionamiento específicas. 


\subsubsection{Marco geológico}

El Observatorio Geofísico de Trelew se encuentra ubicado dentro de la Comarca Norpatagónica, que comprende aquellas porciones extracordilleranas de las provincias de Río Negro y del Chubut que se sitúan en líneas generales al $\mathrm{S}$ de los ríos Limay y Negro, al E del margen serrano que bordea la Cordillera Patagónica y aproximadamente al $\mathrm{N}$ del curso medio e inferior del río Chubut, extendiéndose hasta la costa atlántica, las unidades morfoestructurales se observan en la Fig. 5.1.

Esta unidad geoestructural representa un "área positiva" a partir de tiempos neopaleozoicos, considerándosela un "neocratón" post-precámbrico. Esto está corroborado por los descubrimientos de fósiles marinos paleozoicos en Sierra Grande y el hallazgo de sedimentitas con escaso o nulo metamorfismo al Oeste de Valcheta, dentro de un ambiente metamórfico (Stipanicic, 1976).

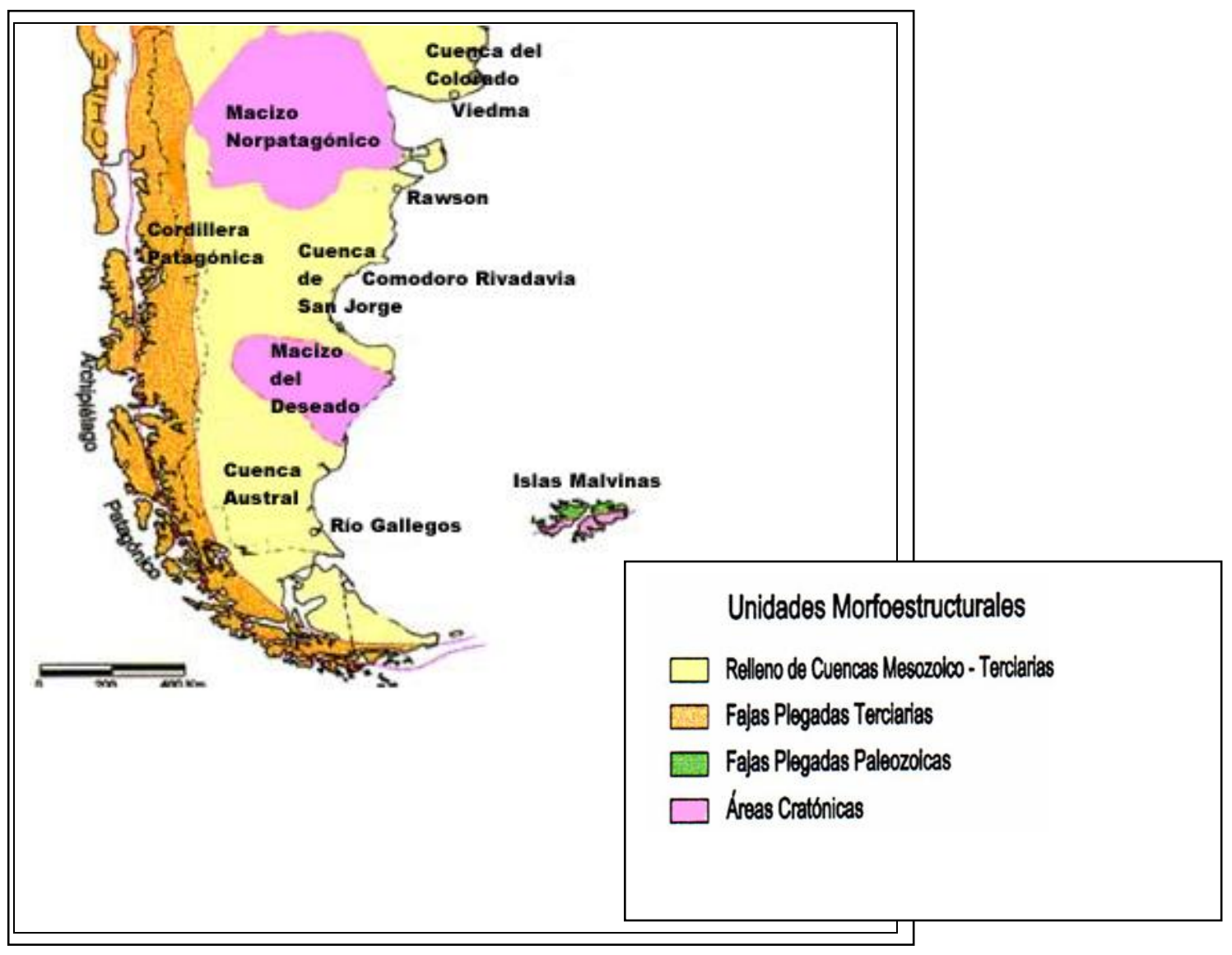

Figura 5.1: Características morfoestructurales de la región Patagónica agrupadas en provincias geológicas.

En la porción occidental de la provincia de Chubut y la zona $\mathrm{N}$ de la provincia de Santa Cruz, se presentan potentes espesores sedimentarios por debajo de las 
rocas efusivas jurásicas, que en la zona oriental, se asientan directamente sobre un "basamento" integrado por metamorfitas y rocas graníticas (Lesta, 1976).

En la región central y nororiental de la provincia de Chubut la cobertura sedimentaria está perturbada con un estilo estructural distinto al resto de la provincia y con un grado de deformación mucho menor. Se trata de estructuras menos relevantes asociadas a las fracturas de basamento o bien corresponden a asentamientos de términos del Grupo Chubut, condicionados por un acentuado relieve preexistente labrado en la irregular superficie de la meseta porfídica del Grupo Lonco Trapial o sus equivalentes (Chebli, 1978).

\subsubsection{Instalación de instrumental de corto período}

Dentro del predio del Observatorio Geofísico, en las proximidades de un pilar fundamental de coordenadas $\varphi=43^{\circ} 15^{\prime} 52.5^{\prime \prime} \mathrm{S}$; $\lambda=65^{\circ} 22^{\prime} 41.5^{\prime \prime} \mathrm{W}$ a una altura de $21 \mathrm{~m}$ sobre el nivel del mar, se encuentran ubicadas 5 casillas que tienen diversos usos, fundamentalmente para Geomagnetismo, dentro de una de las cuales se colocó un sismómetro, para decidir la posible instalación de instrumental permanente (Sabbione et al., 1998).

El sensor utilizado fue un LE-3Dlite Lennartz Electronic, que es básicamente un geófono activo tri-direccional con frecuencia de corte de $1 \mathrm{~Hz}$, conectado a un adquisidor de datos AD7710X3 (ver 3.2.4; 4.2.4)

En otra de las casillas, a 50 metros aproximadamente, se instaló una laptop, en la cual corría el programa de adquisición y archivo, conectados a una alimentación de $12 \mathrm{~V}$ y $220 \mathrm{~V}$ para evitar la suspensión de la registración frente a eventuales cortes en el suministro de energía.

Como sistema de adquisición se utilizó el programa AD7710H.exe y para la visualización y extracción de eventos el programa BANDAS (ver 4.2.4).

Algunos de los registros obtenidos en esa campaña (tabla 5.1) se muestran en las figs. 5.2 y 5.3 , con sus espectros y los espectros del mismo registro en cada caso obtenido en LPAD para la componente vertical (Sabbione et al, 1998).

\begin{tabular}{|c|c|c|c|c|c|c|c|}
\hline Fecha & Lat. $\left({ }^{\circ}\right)$ & Long. $\left({ }^{\circ}\right)$ & Prof. $(\mathrm{km})$ & Mag. $(\mathrm{mb})$ & Dist.a LPA & Dist.aTRL & Lugar \\
\hline $02 / 09 / 97$ & $3.887 \mathrm{~N}$ & $75.740 \mathrm{~W}$ & 222 & 6.4 & $42^{\circ} 07$ & $47^{\circ} 86$ & Colombia \\
\hline $04 / 09 / 97$ & $26.535 \mathrm{~S}$ & $178.322 \mathrm{E}$ & 618 & 6.3 & $98^{\circ} 97$ & $89^{\circ} 23$ & Sur Is. Fiji \\
\hline
\end{tabular}

Tabla 5.1: datos de sismos registrados en Trelew durante instalación de instrumental de corto período, 

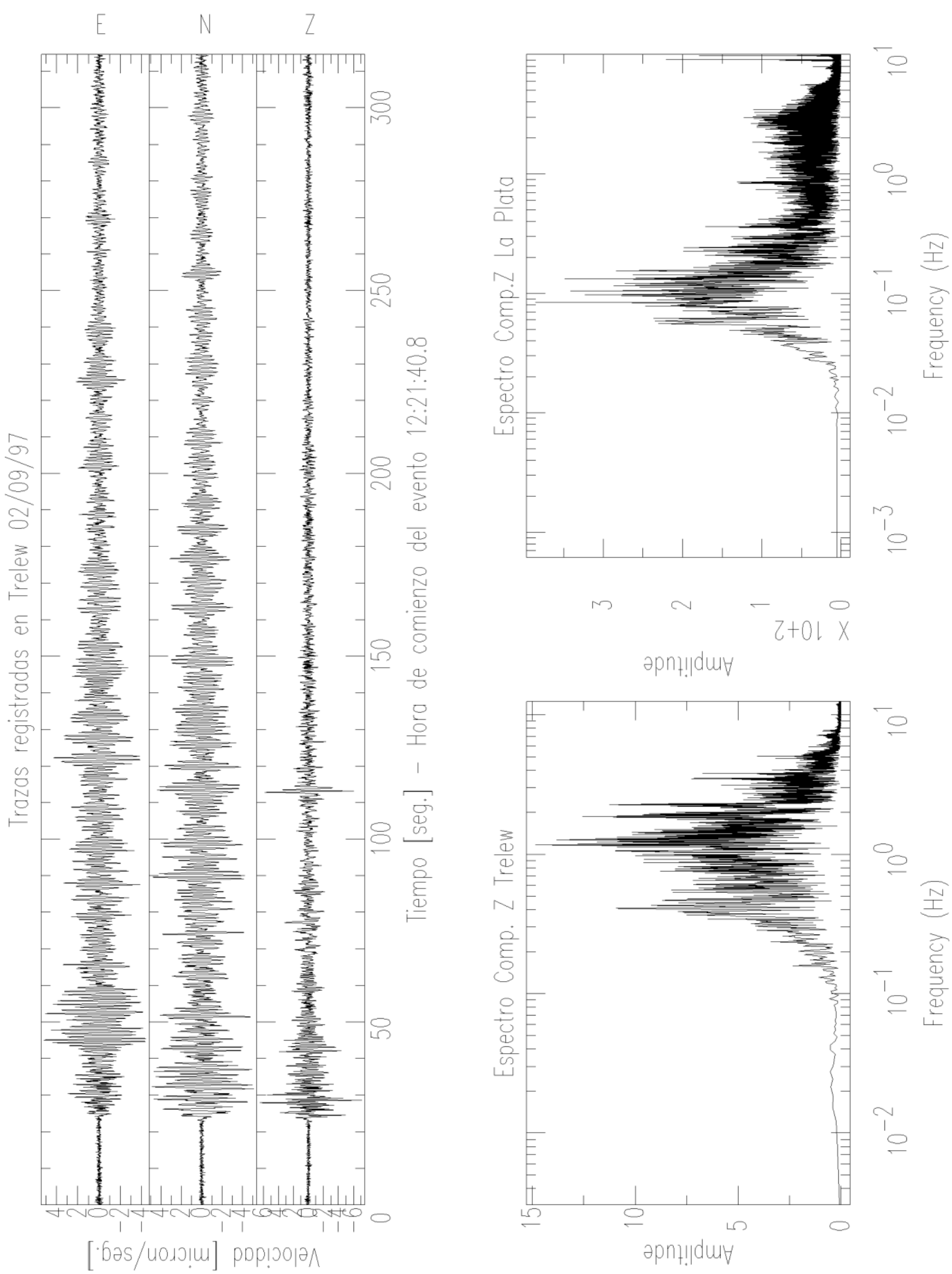

Figura 5.2: Registro del sismo en Colombia obtenido en Trelew y espectros de las comp. Z en LPAD y Trelew. 

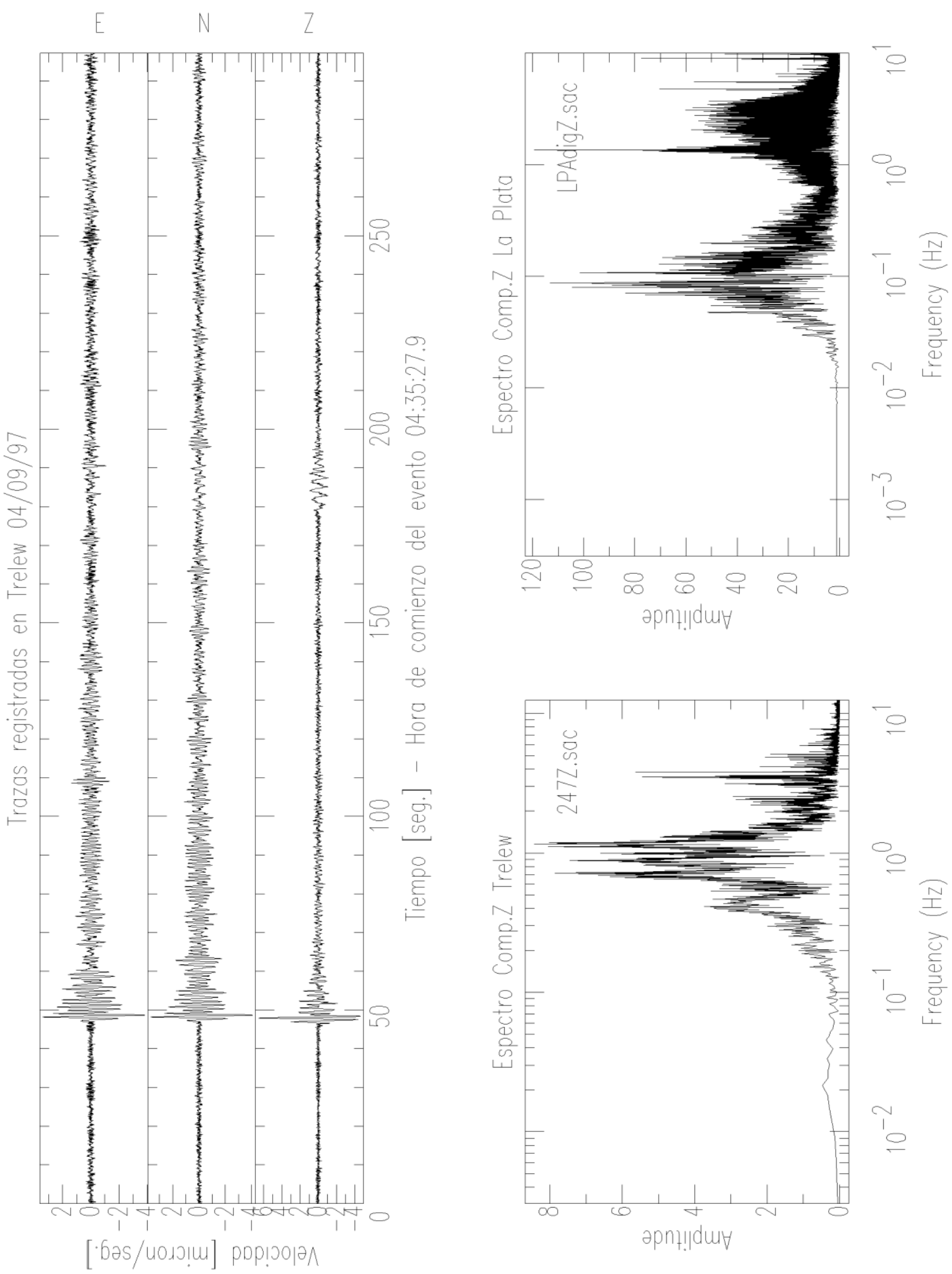

Figura 5.3: Registro del sismo en las Is. Fiji obtenido en Trelew y espectros de las comp. Z en LPAD y Trelew. 
El procesamiento de las trazas se realizó bajo ambiente UNIX, usando el SAC, Seismic Analysis Code.

\subsubsection{Análisis del ruido y comparación con La Plata}

Para realizar la determinación del ruido en Trelew se utilizaron muestras tomadas cada hora durante los días 29, 30, y 31/07, 17/08 y 23/09 de 1997.

Se muestra en la fig. 5.4 el ruido en velocidad detectado con el equipamiento utilizado, en Trelew (TRWA) y en La Plata (LPAD), (Sabbione y otros, 1998).

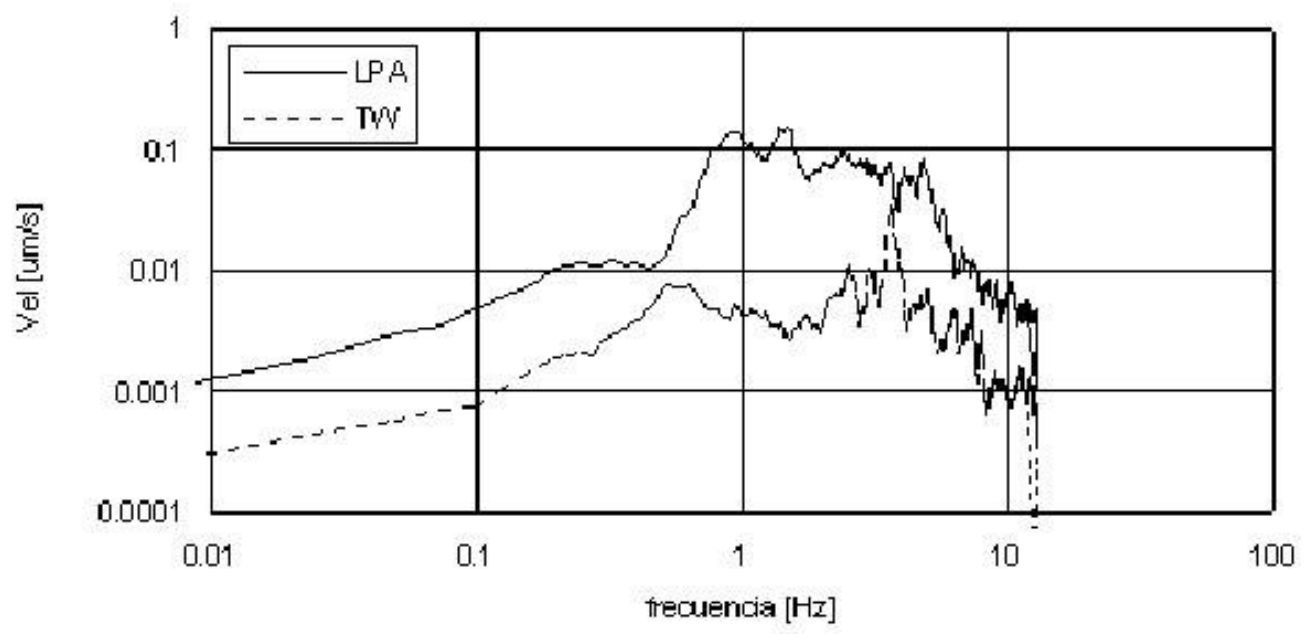

Figura 5.4: Ruido comparativo en las estaciones de Trelew y La Plata (Sabbione y otros, 1998)

De este gráfico se desprende que el ruido en Trelew es diez veces menor que en La Plata y que en Trelew se observa una línea espectral en 3,6 Hz, de amplitud considerable, cuya fuente generadora probablemente haya sido un compresor o bomba de líquidos industrial cercanos.

Este análisis llevó a la conclusión que era factible la instalación de instrumental permanente, y teniendo en cuenta la influencia de los vientos de la región, se decidió la construcción de un albergue subterráneo para los sensores de banda ancha que se instalarían más adelante.

\section{2 - Estación sismológica TRWA}

\subsubsection{Características generales}

La Estación de la UNLP en Trelew, TRWA, donde funciona el Observatorio Geofísico dependiente de la FCAGLP, se encuentra ubicada en un predio de $44 \mathrm{Ha}$, 
aproximadamente a $8 \mathrm{~km}$ de la ciudad de Trelew, sobre la Ruta Provincial 25, que une las ciudades de Trelew y Gaiman. Las coordenadas son $\varphi=43^{\circ} 15^{\prime} 54.8^{\prime \prime} S \lambda=$ 65²2'38.4" W altura sobre el nivel del mar: 47 metros +/- 3 m (Fig.5.1). La estación con instrumental banda ancha ha estado registrando en forma continua, desde abril de 1999, operada por un geofísico residente.

\subsubsection{Instalación de instrumental permanente}

Instalación de los sensores

Para alojar los sensores se construyó un recinto subterráneo de baja profundidad en un lugar ubicado a 50m en dirección SE desde la Casilla de Control, en una pequeña elevación del terreno (Pinciroli y otros, 2000).

Las medidas interiores del recinto terminado son: $1.90 \mathrm{~m} \times 1.20 \mathrm{~m}$ por $1.40 \mathrm{~m}$ de alto; en el piso se dejó el suelo natural con el objeto de absorber posibles filtraciones de agua de lluvias. Se construyó un pilar de cemento con una base de $0.9 \mathrm{~m} \times 0.9 \mathrm{~m}$, sobresaliendo $20 \mathrm{~cm}$, su cara superior está a $2.00 \mathrm{~m}$ debajo del nivel del terreno.

Las paredes se construyeron con ladrillo hueco y la losa superior de hormigón armado se realizó en dos capas, con poliestereno expandido de $20 \mathrm{~mm}$ como aislante térmico entre ellas. El recinto se vinculó a la "Casilla de control" mediante 2 caños plásticos para conducir los cables de señal y alimentación de $12 \mathrm{~V}$ y $220 \mathrm{~V}$ por separado. La construcción finalizó en febrero de 1999.

El pilar se construyó con $50 \%$ de arena fina y $50 \%$ de cemento, bien vibrado
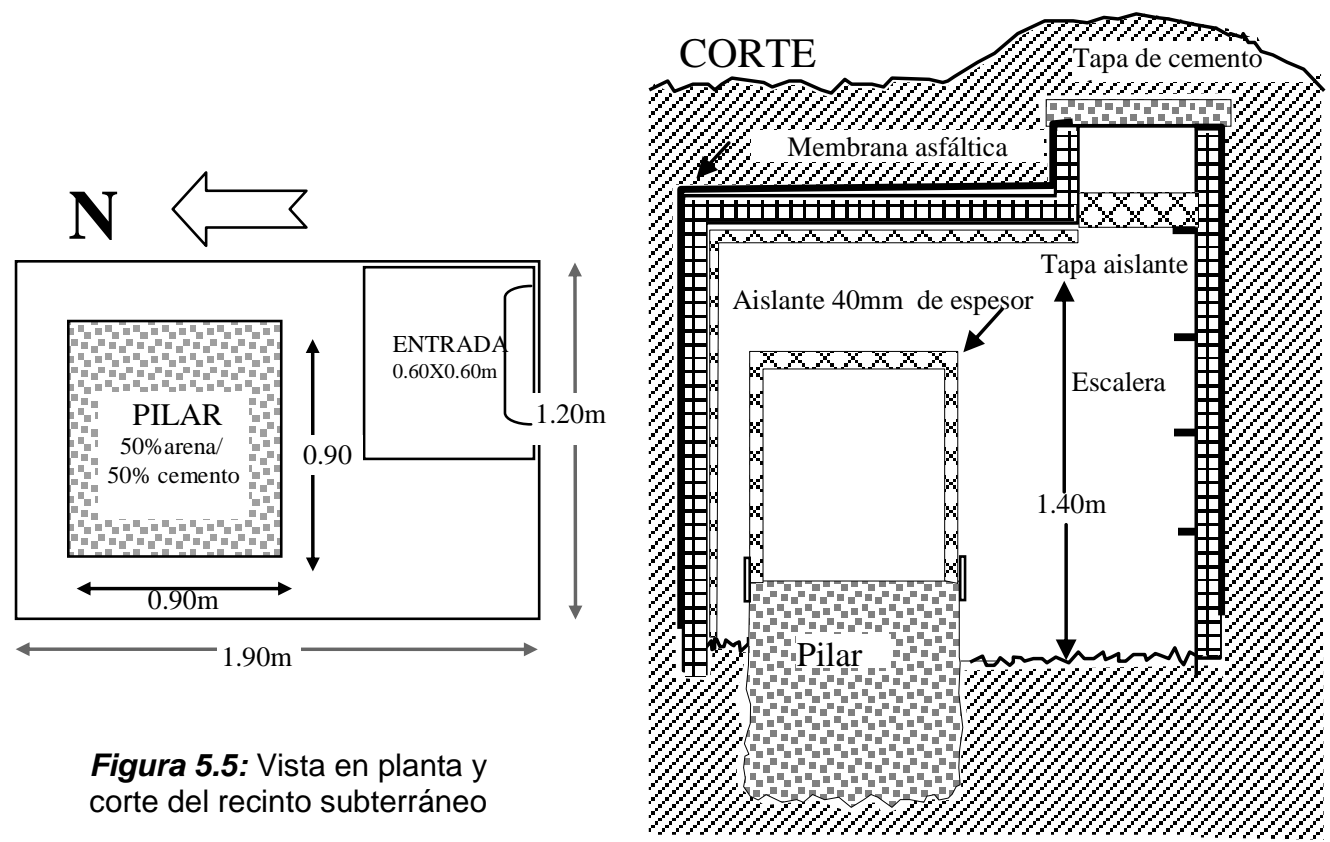

Figura 5.5: Vista en planta y corte del recinto subterráneo 
para que no contenga burbujas de aire, no se agregó hierro ni piedra para evitar la posible producción de ruido sísmico a causa de los diferentes coeficientes de dilatación (Uhrammer y otros, 1998). Se dejó fraguar durante un mes, antes de retirar el encofrado.

El acceso se realiza a través de una boca de $0.6 \mathrm{~m} \times 0.6 \mathrm{~m}$, que se mantiene tapada con una laja de hormigón cubierta con unos $0.3 \mathrm{~m}$ de tierra, para evitar el vandalismo. Ver corte y planta en la Fig. 5.5.

Con la finalidad de aislar los sensores de los cambios de temperatura se construyó una caja usando láminas de poliestireno expandido extruído de $40 \mathrm{~mm}$ de espesor con una densidad de $32 \mathrm{Kg} / \mathrm{m} 3$ ("Polyfan"), recubierto con hoja de aluminio brillante exterior, esta caja tiene las medidas del pilar y cubre los 3 sensores.

\section{Instrumental}

Sismógrafo: PDAS100 Geotech

Teledyne Sensores: BB13 sismómetros banda ancha, Geotech Teledyne (USA). (Fig.

Tiempo: GPS Acutime Trimble. (USA)

Instrumental cedido por el Programa

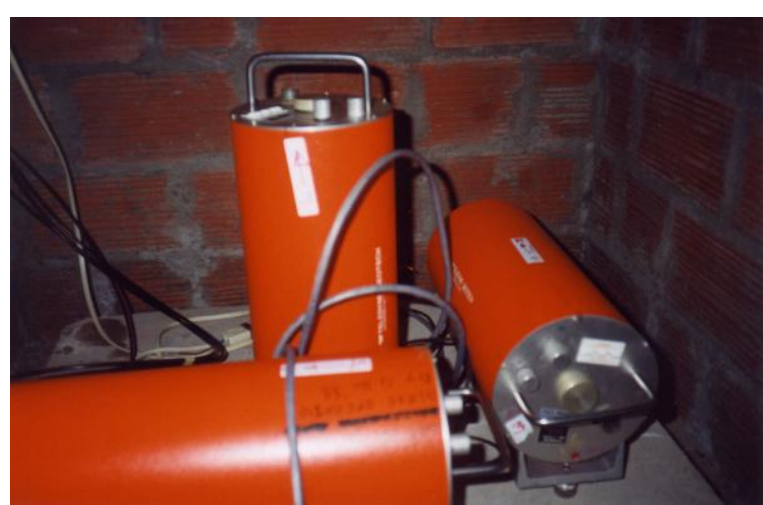

Figura 5.6: Sensores BB13.

Nazionale di Ricerche in Antartide (PNRA)

de Italia en el marco de un acuerdo.

Las características se resumen en el esquema:

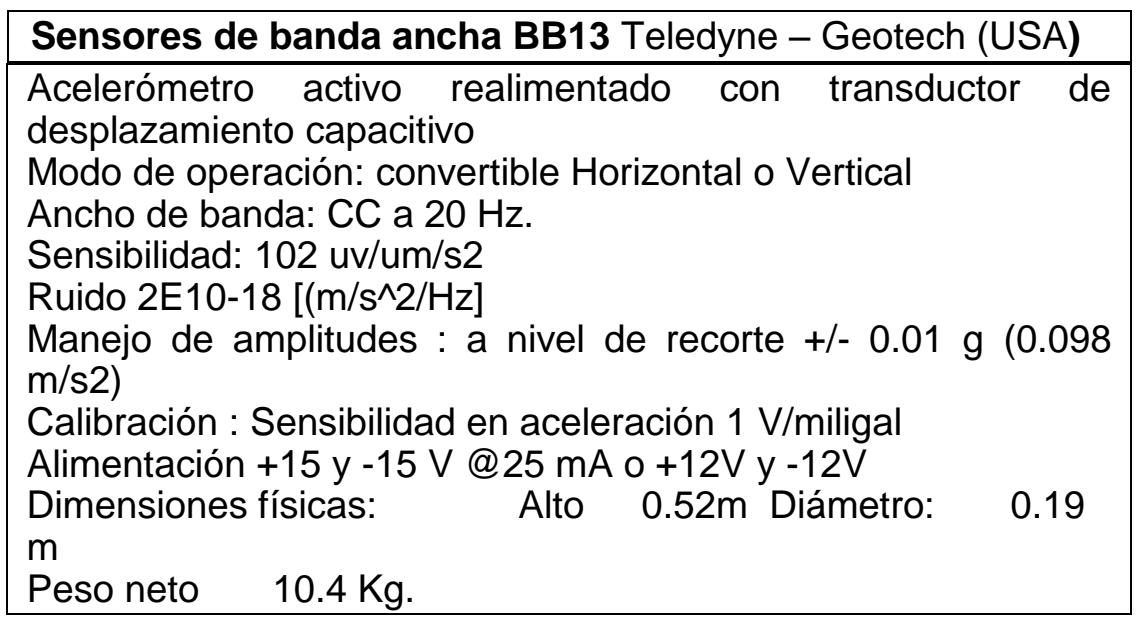




\begin{tabular}{|l|}
\hline $\begin{array}{l}\text { Adquisidor de datos sísmicos PDAS } 100 \text { Teledyne-Geotech } \\
\text { (USA) }\end{array}$ \\
\hline 3 canales mas 1 entrada auxiliar16 bits, rango dinámico \\
extendido hasta $132 \mathrm{~dB}$ por ganancia automática, \\
Frec. muestreo $1000 \mathrm{mps}$ básico, 12 frecuencias de muestreo \\
para elegir \\
Procesador de señales DSP CMOS 16 bits \\
Reloj de tiempo real con cristal compensado \\
Receptor GPS Trimble \\
Memoria de estado sólido $8 \mathrm{Mb}$ \\
Salida datos puertos serie y paralelo \\
Señales de calibración sinusoidales, pulso y ruido aleatorio \\
Alimentación $10-14 \mathrm{~V} \mathrm{CC} 400 \mathrm{~mA}$ \\
Peso neto $10.4 \mathrm{Kg}$. \\
\hline
\end{tabular}

Los sismómetros de banda ancha fabricados por Geotech - Teledyne (USA) en 1988, son una adaptación de los sismómetros de corto período $\mathrm{S} 13$ a los que se actualizó agregando un transductor de desplazamiento capacitivo en un lazo de realimentación electrónico para la bobina principal, lo que logra un instrumento que detecta aceleraciones sobre un ancho de banda desde corriente continua hasta $20 \mathrm{~Hz}$.

Antes de su instalación definitiva se determinaron las constantes, con pesas en la componente vertical y con plano inclinado en las horizontales; se verificó la respuesta en toda la banda con señal de ruido generada por el PDAS100 (Fig. 5.7).

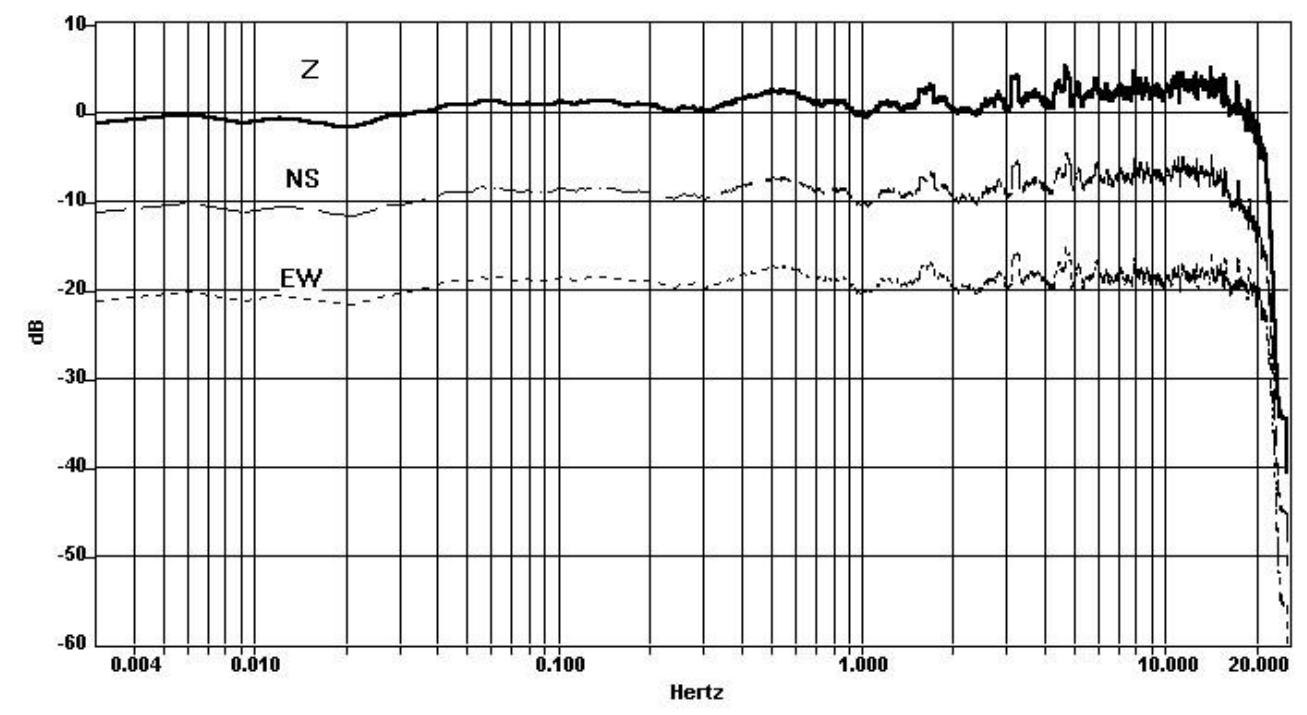

Figura 5.7: Curvas de respuesta en frecuencia de los 3 sensores obtenidas con señal de calibración de ruido de banda ancha, se usó el equipo completo con una frecuencia de muestreo de $100 \mathrm{sps}$. Las curvas se desplazaron intencionalmente $10 \mathrm{~dB}$, para su mejor visualización (Pinciroli y otros, 2000). 
En una casilla cercana se dispuso el adquisidor, un PDAS100 Teledyne Geotech (USA) de 1988, con software de 1992 con poca memoria que se aumentó con el agregado de un disco extraíble ZIP de 100 Mbytes. Se ajustó la configuración de disparo por evento en el canal Z filtrado y se adoptó una frecuencia de muestreo de 20 mps. El tiempo se mantuvo por GPS hasta agosto de 1999 en que el receptor dejó de funcionar por la falla conocida como defecto WNRO (Week Number Roll Over) y desde allí con el reloj interno de tiempo real.

Se proveyó una alimentación ininterrumpible (UPS), que asegura una autonomía de 20 horas, conectada a la red domiciliaria local. También se instaló una PC 486 auxiliar utilizada para modificar los parámetros de adquisición y permitir la visualización y extracción de los datos.

\section{Parámetros:}

Frecuencia de muestreo:20 mps.

Resolución (CAD): 16 bits.

Operación: Por disparo con algoritmo STA/LTA.

Sensibilidad: 3.3 cuentas $\mathrm{s}^{2} / \mu \mathrm{m}$.

\section{El comportamiento térmico del conjunto}

Disponiendo de dos sensores de temperatura: uno en el recinto y otro en la

BB13 Posición de la masa vs. Temperatura

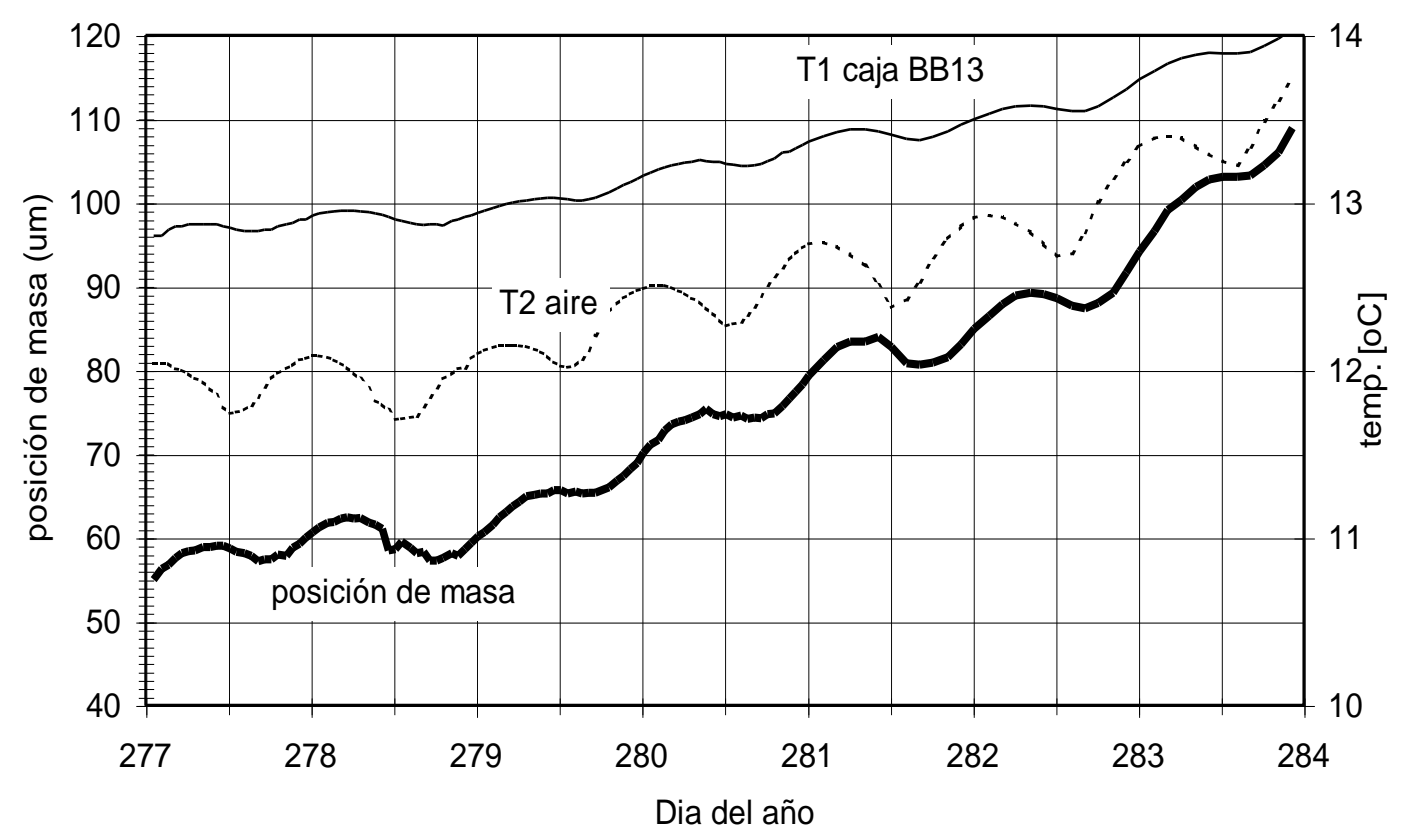

Figura 5.8: Comportamiento térmico del conjunto. 
caja de la componente vertical, se tomaron medidas simultáneas de temperatura y del movimiento de la masa.

En la fig. 5.8 se representa una semana de registro, se observa el modo en que la masa acompaña con fidelidad a la fluctuación de la temperatura de la caja del instrumento (T1).

La ondulación en las curvas corresponde a la fluctuación térmica diaria. La temperatura de la caja del BB13 es algo mayor que la del aire a causa de la potencia eléctrica de alimentación de la electrónica interior; la menor amplitud de la oscilación y el defasaje en atraso es producido por el filtro pasa bajos resultante de la aislación térmica y la masa del instrumento.

Como se puede ver, la posición de la masa del sensor acompaña fielmente a la variación de temperatura; estas curvas se usaron para determinar la constante de corrimiento térmico del sensor (para la unidad BB13 en vertical № serie 033) resultando un valor de $\mathrm{Kt}=329\left[\mu \mathrm{m} / \mathrm{s}^{2}\right]+/-20$.

\section{3 - Uso de la estación.}

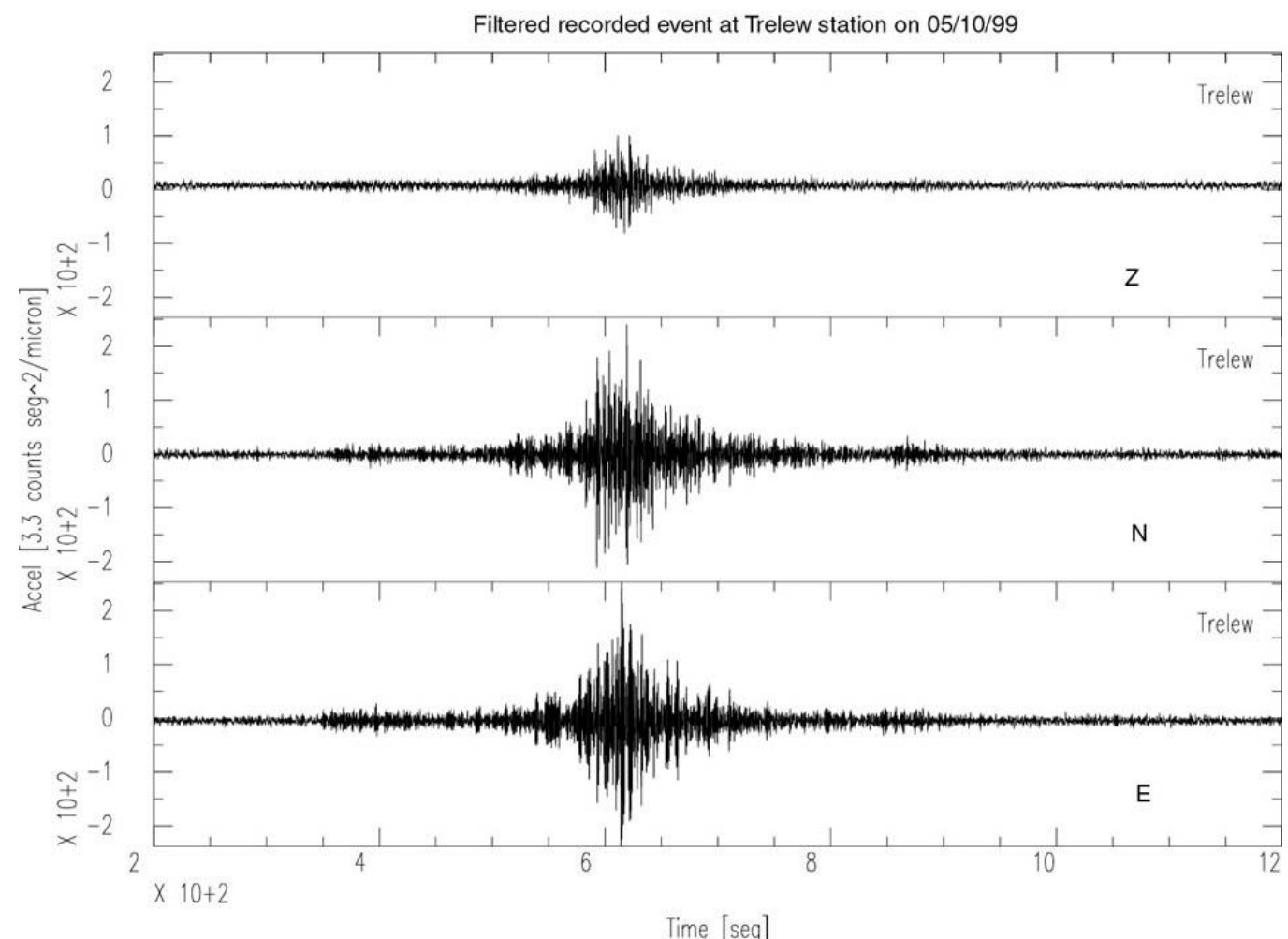

Figura 5.9: Ejemplo de sismograma superficial registrado en TRWA (de Sabbione y otros, 2000). 


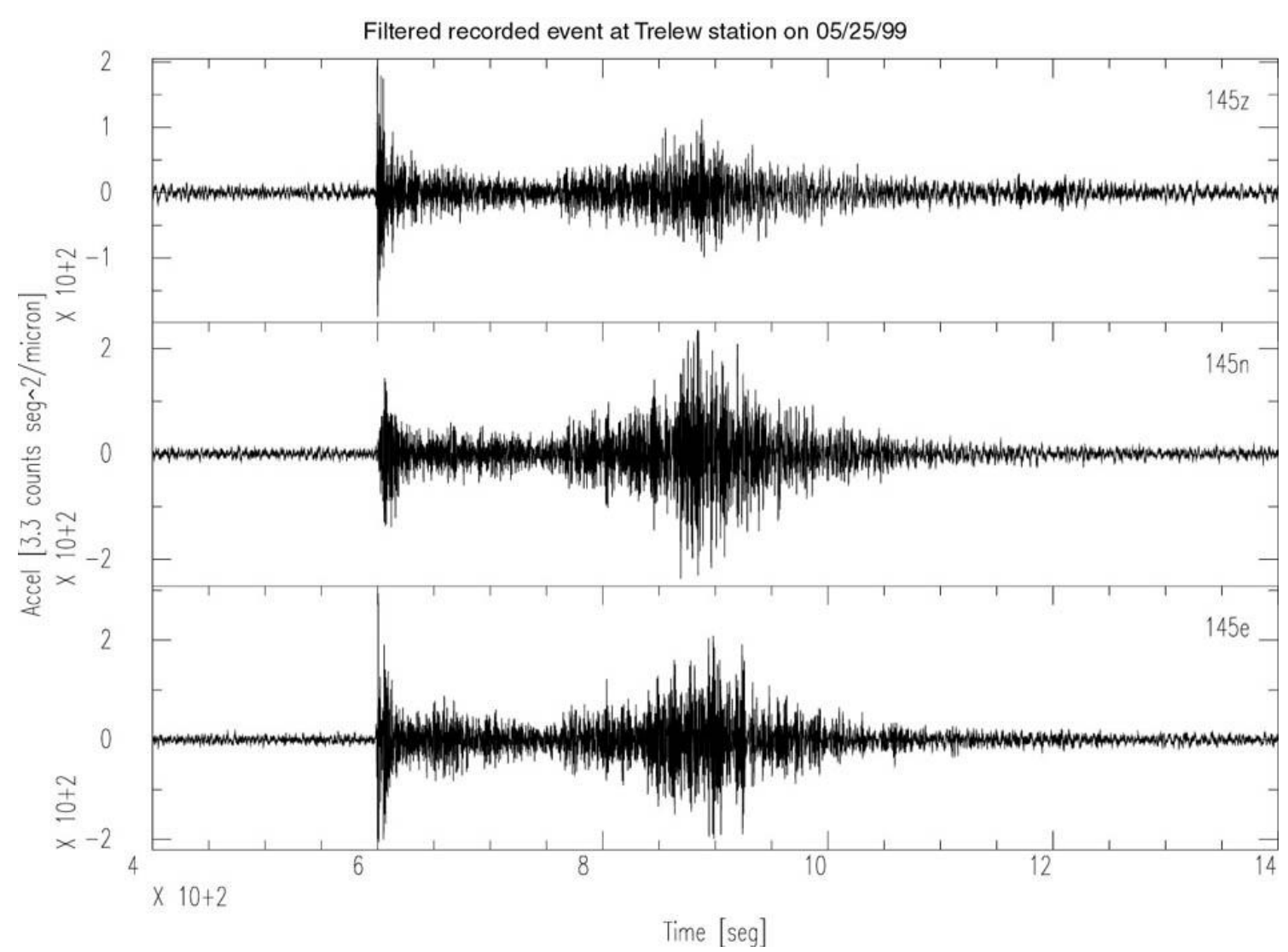

Figura 5.10: Ejemplo de sismograma de profundidad intermedia registrado en la estación TRWA (de Sabbione y otros, 2000a).

Se presentan en las Figs. 5.9 y 5.10 dos sismogramas obtenidos en la estación, correspondientes a los siguientes eventos:

\begin{tabular}{|c|c|c|c|c|c|c|c|}
\hline Fecha & Hora de origen & Lat. $(\stackrel{\circ}{)}$ & Lon. $(\stackrel{\circ}{)}$ & Prof, $(\mathrm{km})$ & Mag.Mb & Dist. Epic. $\Delta^{\circ}$ & Región \\
\hline 05/10/99 & $08: 47: 25.0$ & $30.39 \mathrm{~S}$ & $69.16 \mathrm{~W}$ & 65 & 5.2 & $13^{\circ} 2$ & Chile-Arg. \\
\hline $05 / 25 / 99$ & $16: 42: 05.3$ & $27.93 \mathrm{~S}$ & $66.93 \mathrm{~W}$ & 169 & 5.3 & $15^{\circ} 35$ & Catamarca. \\
\hline
\end{tabular}

Tabla 5.2: Parámetros focales de los eventos seleccionados procesados con SAC(Tapley, 2000)

Dado que la capacidad de almacenaje de datos del PDAS 100 es pequeña, aún con el disco extraíble (ZIP 100 MB), se consideró insuficiente para optar por el registro continuo. Se decidió, entonces, registrar con disparo por evento usando la conocida relación STA/LTA; se inició con una configuración que se fue modificando de acuerdo a los resultados obtenidos y a los espectros de los sismos registrados. Con 
posterioridad, a efectos de poder tener un registro continuo se instaló un adquisidor de datos AD7710X3 (3.2.4), actualmente en uso.

\section{4 - Ruido sísmico en la estación}

\subsubsection{Aspectos del ruido sísmico en aceleración}

Los fuertes vientos en la región son la principal fuente de ruido en la estación Trelew. Éste ha sido estudiado considerando registros diurnos, nocturnos y ventosos.

En la Fig. 5.11 se muestran algunas curvas elegidas para representar el conjunto (se expresan en unidades de aceleración). Estas curvas fueron obtenidas calculando el espectro y suavizando por promedio móvil de 20 puntos utilizando la información del tramo anterior a los sismos en los eventos registrados, y con algunos registros especiales tomados a 50 y 100 mps. Se representa también la curva del modelo de bajo ruido (LNM) esquematizada con segmentos de recta (Peterson, 1980).

Analizando la distribución espectral se pueden mencionar tres zonas de interés: - de los $0.1 \mathrm{~Hz}$ a $1 \mathrm{~Hz}$ : es una zona muy variable en amplitud pues depende de la actividad oceánica, la estación dista unos $40 \mathrm{~km}$ del mar. Se observan los dos picos correspondientes a las ondas oceánicas el de menor frecuencia, y a las ondas estacionarias generadas a partir de ellas, en la frecuencia doble (Aki-Richards, 1980).

Las frecuencias de estos picos resultan el doble de las allí mencionadas (aproximadamente 6 y 3 segundos), ver parte superior de fig 5.11 .

- de 1 a $2 \mathrm{~Hz}$ : se observa un pico en las componentes horizontales, ver parte inferior de fig. 5.11, con una altura entre 4 y 5 veces el valor de la componente vertical, debido a las condiciones meteorológicas locales, especialmente afectadas por el viento y muy evidentes por la poca profundidad del recinto (Aki-Richards, 1980).

- entre 2 y $10 \mathrm{~Hz}$ : su forma y amplitud depende del viento y de la actividad de la ciudad cercana. 

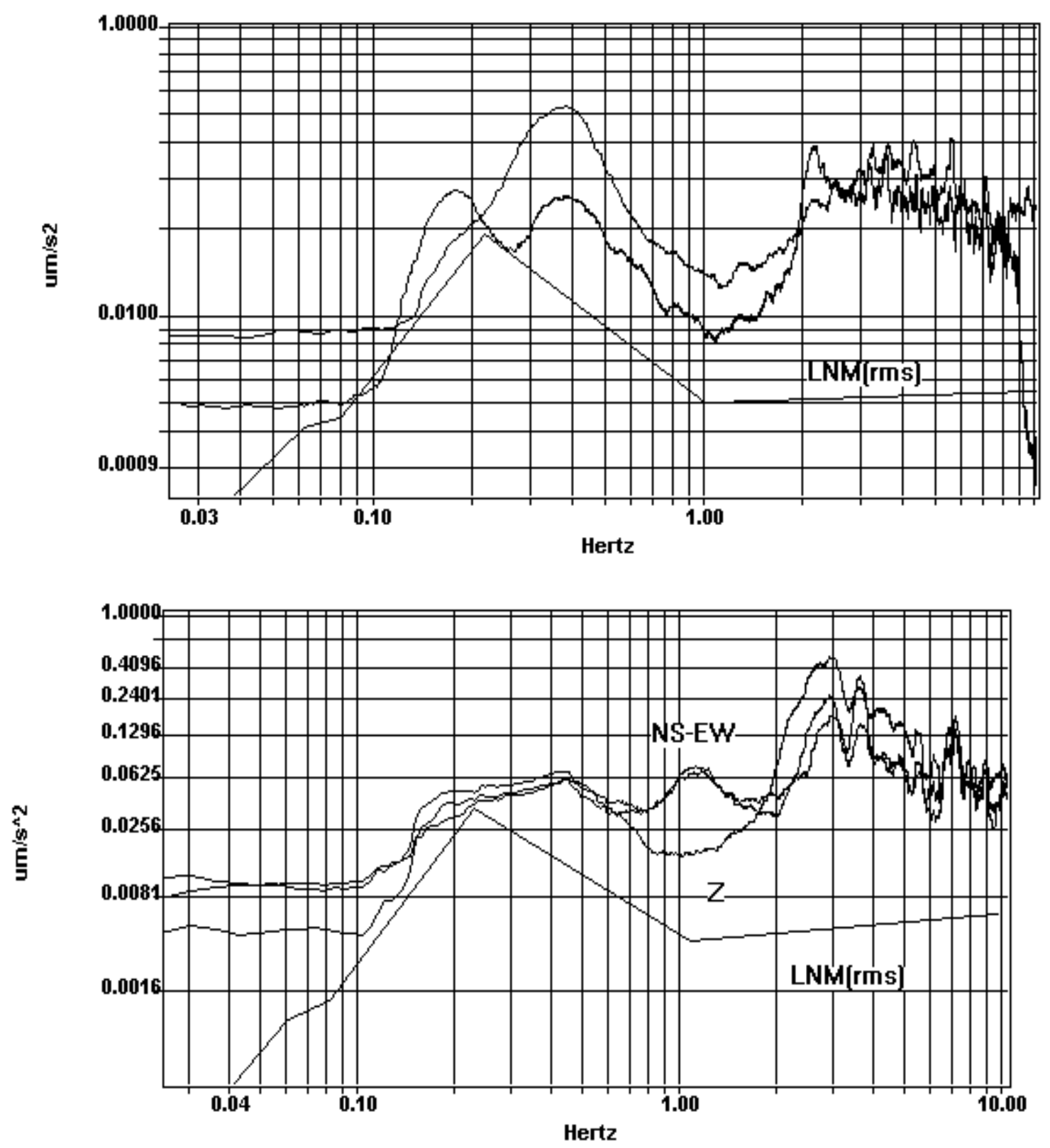

Figura 5.11: (arriba) Ruido en la componente vertical mostrando dos niveles (abajo) Comparación de componentes horizontales y verticales de un mismo día LNM curva del modelo de ruido mínimo, Peterson,1980. 


\subsubsection{Ruido en función del análisis de densidad espectral de potencia en aceleración.}

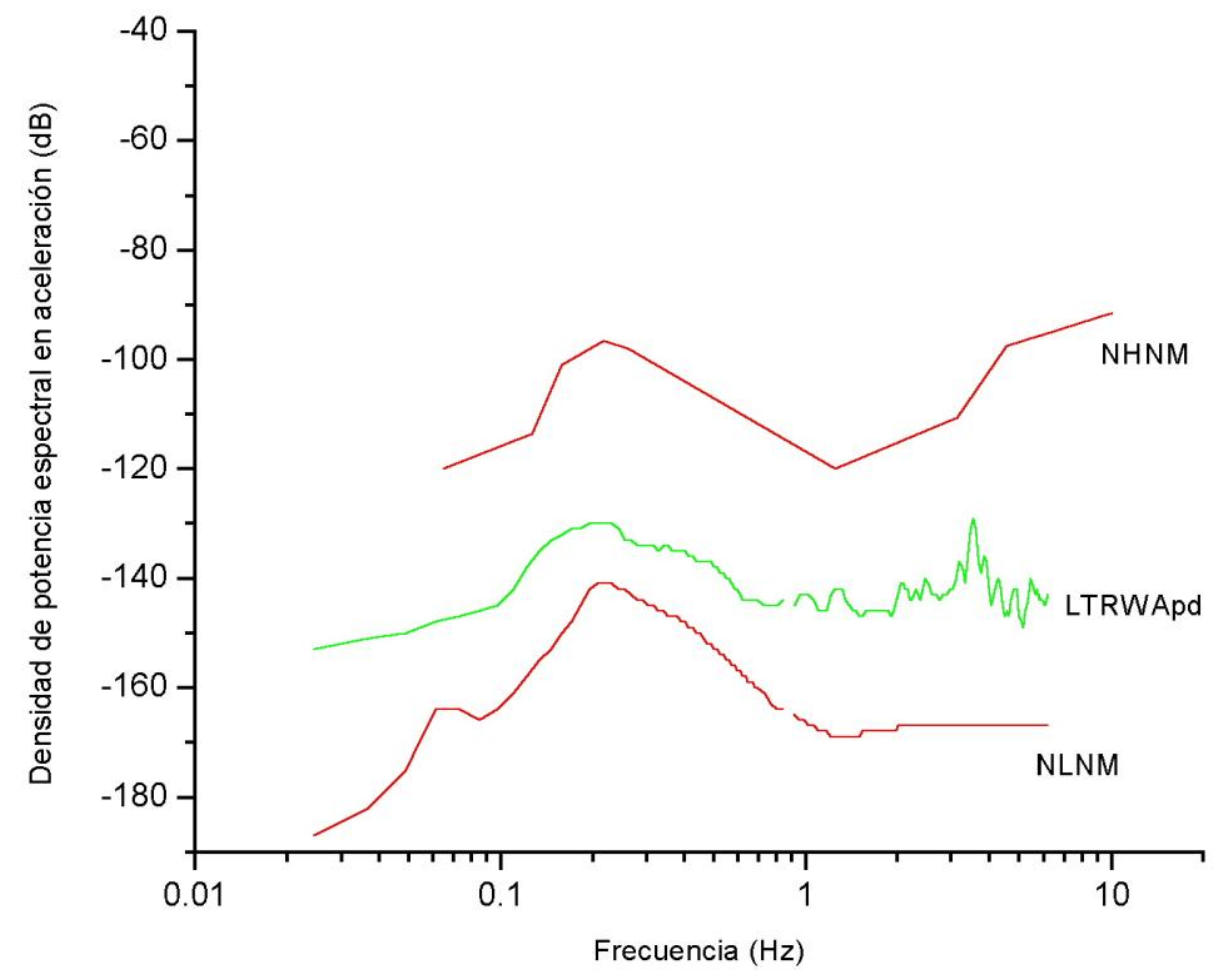

Figura 5.12: Ruido en la estación sismológica TRWA expresado en densidad de potencia espectral, comparado con las curvas de ruido NLNM-NHNM de Peterson (1993)

Se puede ver en este gráfico que la estación sismológica Trelew posee un nivel de ruido que se encuentra muy por debajo del nivel alto de ruido NHNM en toda la banda de frecuencias analizada, lo que hace de esta estación una estación de buena calidad para el registro de sismos regionales y lejanos.

A pesar que esta estación se encuentra ubicada en un área muy ventosa, haber instalado los sensores en un recinto por debajo del nivel del suelo ha permitido mejorar sensiblemente la calidad de los registros, tal como se desprende de la figura 5.12 . 


\section{Capítulo 6 \\ ESTACION SISMOLÓGICA DSPA \\ Ea DESPEDIDA,TIERRA DEL FUEGO, ARGENTINA}

\section{1 - Antecedentes}

\subsubsection{Breve reseña}

En el marco del proyecto para el estudio de la Geofísica y Geodinámica de la Isla de Tierra del Fuego, (PICT 385 año 1997, Agencia Nacional de Promoción Científica y Tecnológica) a partir del año 1998 se iniciaron estudios a efectos de instalar instrumentación sismológica.

En el mes de marzo de 1999 se realizó un viaje a la Isla a efectos de evaluar los posibles lugares de emplazamiento de la estación sismológica. En colaboración con investigadores del Centro Austral de Investigaciones científicas (CADIC), Ushuaia, se realizó la selección del sitio, que se encuentra ubicado en la Estancia Despedida, $50 \mathrm{~km}$ al sudoeste de la ciudad de Río Grande, en una cresta de dirección NO-SE, sobre un afloramiento rocoso conglomerado, formación Ea Despedida, correspondiente al Eoceno-Oligoceno (aprox. 45 millones de años), a una distancia de la casa principal de la Estancia del orden de $500 \mathrm{mts}$.

En junio de 1999 (1er etapa) comenzó la registración, atendida por personal de la Estación Astronómica Río Grande., utilizando un sensor de corto período y dependiendo de la UNLP. Dicha estación constaba de un sensor Lennartz LE-3Dlite de $1 \mathrm{~Hz}$, un adquisidor de tres canales con Conversor Analógico Digital de 16 bits con registro continuo a $25 \mathrm{mps}$ (CSIC, Consejo Superior de Investigaciones Científicas Madrid, España) y una PC laptop para la operación, control y el almacenamiento de los datos. La potencia era suministrada por paneles solares de 50 watts.

Esta estación operó hasta fines del año 2002 en que fuera reemplazada por una estación banda ancha de última generación (2da etapa) en el marco de un Convenio de cooperación entre la UNLP y el Istituto Nazionale di Oceanografia e di Geofísica Sperimentale- INOGS, Italia, Anexo № 1, formando parte de la red ASAIN, Antarctic Seismographic Argentinian Italian Network.

Las coordenadas de la estación DSPA son:

$$
\varphi=53^{\circ} 57^{\prime} 13.046^{\prime \prime} \mathrm{S} \quad \lambda=68^{\circ} 16^{\prime} 00.521^{\prime \prime} \mathrm{W} \text {. }
$$

Red ASAIN: En el curso de los años 1992 al 1996, con el objetivo de profundizar el conocimiento de la tectónica y de la geodinámica de esas regiones, inhóspitas, pero 
extremadamente interesantes desde el punto de vista científico, (Russi et Febrer, 2001) se instalaron tres estaciones sismográficas de banda ancha de tres componentes. En la zona austral de Sudamérica USHU, Ushuaia, Tierra del Fuego y en las regiones Antárticas ESPZ, Esperanza y ORCD, Orcadas, que constituyen el límite meridional del Mar de Scotia. Estas estaciones forman parte de la actividad prevista por el Proyecto "Sismología a larga banda nella regione del Mare di Scotia", Programma Nazionale di Ricerca in Antartide, Italia, y el Instituto Antártico Argentino (IAA). En una primera etapa esas tres estaciones constituían la Red Sismográfica Antártica Italo Argentina, ASAIN, (Febrer, 2002), que a partir de fines del año 2002 se ha ampliado con la incorporación de la estación sismológica DSPA.

Los datos adquiridos por las estaciones ASAIN, además de constituir una relevante contribución a la base de datos sismológicos de la región sub-antártica del Mar de Scotia, revisten particular importancia para el estudio de la sismicidad local en Tierra del Fuego.

\subsubsection{Marco geológico y geodinámico}

En la Isla Grande de Tierra del Fuego el ambiente tectónico es originado por la conjunción entre las placas litosféricas Sudamericana por el norte y de Scotia por el sur, así como por la apertura del pasaje de Drake y los movimientos de la placa de Scotia. Dicho contacto entre placas es definido por un sistema de fallas transcurrentes que se extienden al lo largo de la Cordillera Fueguina y su continuación hacia el este por la Dorsal de Scotia Norte, conocido como sistema de fallas Magallanes-Fagnano (Cunningham y otros, 1995). Las evidencias geológicas (Fig. 6.1), así como evidencias geodésicas y sismológicas indican que este sistema de fallas es uno de los componentes más activos del borde de placas mencionado (Cunningham, 1993, Olivero y otros, 1995, Olivero, 1997). En efecto, como parte del desarrollo del Proyecto Geofísica y Geodinámica en Tierra del Fuego (PICT97), se llevaron a cabo una serie de campañas geológicas orientadas a afinar detalles sobre la estructura y estratigrafía de la faja comprendida por el sistema de falla Magallanes-Fagnano. Como resultado de dicho estudio se pudo establecer que la distribución regional de unidades estratigráficas del Cretácico-Eoceno sugiere desplazamientos de rumbo levógiros del orden de $20-30 \mathrm{~km}$ a lo largo del sistema de fallas Magallanes-Fagnano; esto evidencia que el régimen transcurrente se mantiene activo, por lo menos, desde el Eoceno cuspidal (Olivero y otros, 2001). 
Se detectaron además evidencias geomorfológicas de actividad tectónica desde al menos el Plioceno hasta la actualidad. Hay signos notorios de que la transcurrencia afectó la estructura previa de la faja cordillerana de Tierra del Fuego, dislocándola a través de desplazamientos de rumbo con componentes compresivos y extensivos. En el sector central de la Isla Grande de Tierra del Fuego, desde el extremo oriental del Lago Fagnano hasta la costa Atlántica, se determinaron una serie de bloques deprimidos y estructuras anticlinales desarrollados al sesgo de la falla transcurrente Magallanes-Fagnano.

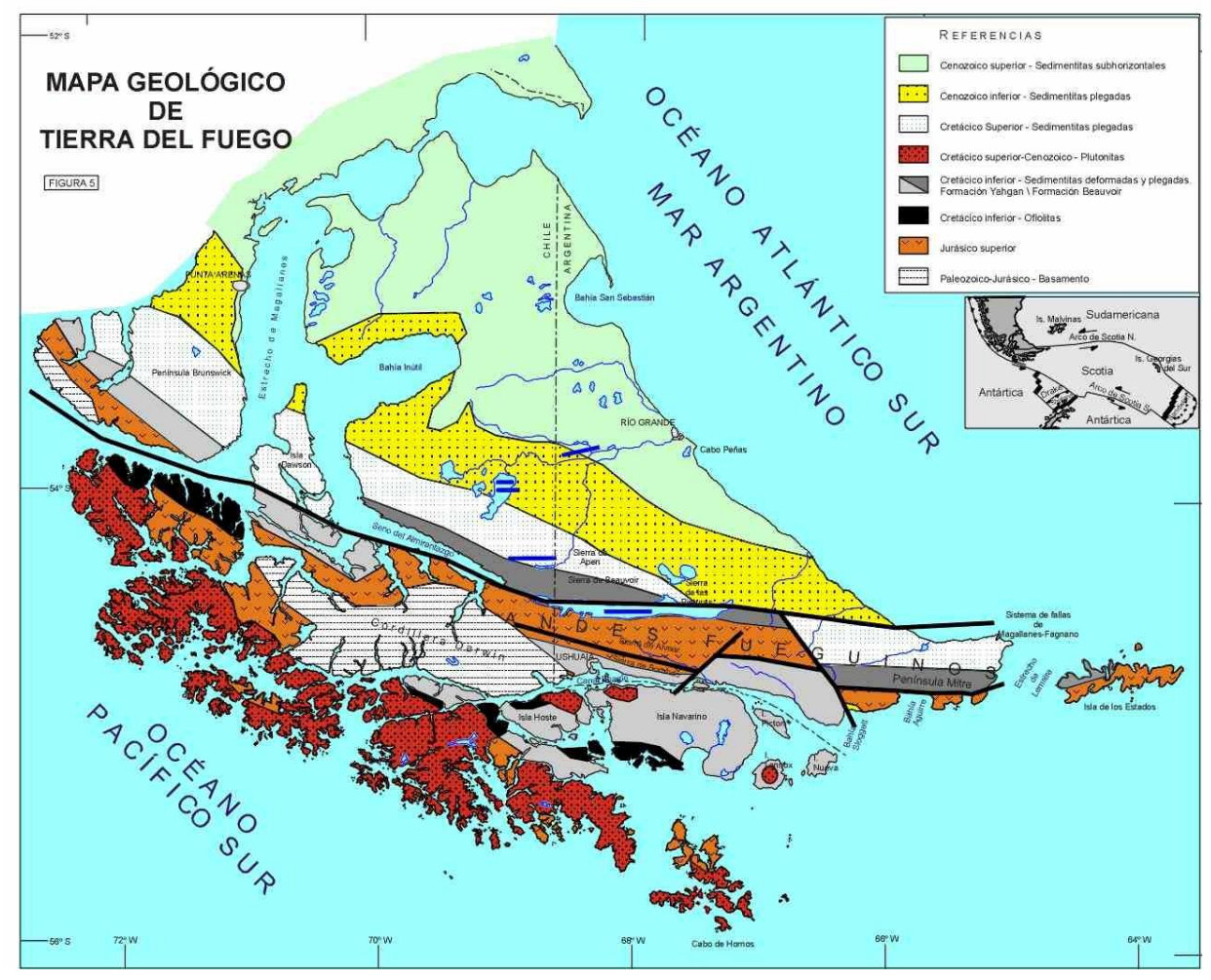

Figura 6.1: Mapa geológico de Tierra del Fuego. (fuente: laboratorio de Geología Andina, CADIC).

Los bloques limitados por fallas normales, evidencian una actividad moderna (neotectónica), marcada por el control estructural que ejercen sobre el desarrollo de las planicies aluviales de los principales ríos, como las de los ríos San Pablo, Irigoyen, Malengueña, Láinez y Turbio, por mencionar los más importantes. Los principales lineamientos así definidos coinciden con la configuración de una zona de cizalla de Riedel con sus dos elementos integrantes, las cizallas sintéticas $(R)$ y antitéticas ( $\left.R^{\prime}\right)$ (Davis y otros, 2000). 


\subsubsection{Marco Sismológico}

La Isla de Tierra del Fuego tiene una importante historia sismológica, incluyendo un evento de magnitud 7.5 ocurrido en el año 1949, tabla 6.1, no obstante lo cual solo recientemente se encuentran disponibles datos instrumentales adecuados para investigar la sismicidad y su relación con la tectónica del área, a partir de los registros de las estaciones sismológicas de Ushuaia (IAA-PNRA) y Despedida (UNLPPNRA).

\begin{tabular}{|c|c|c|c|c|c|c|c|}
\hline $\begin{array}{c}\text { Fecha } \\
(\mathrm{aaaa} / \mathrm{mm} / \mathrm{dd})\end{array}$ & Fuente & $\begin{array}{c}\text { Hora } \\
(\mathrm{GCT})\end{array}$ & $\begin{array}{c}\text { Latitud } \\
(\mathrm{S})\end{array}$ & $\begin{array}{c}\text { Longitud } \\
(\mathrm{W})\end{array}$ & $\begin{array}{c}\text { Prof. } \\
(\mathrm{km})\end{array}$ & Mag. & $\mathbf{N}^{\circ}$ Estac. \\
\hline 18790202 & C. Lomn & $03: 30$ & & & & & \\
\hline 19290607 & ISS & 000500 & 53.0 & 65.5 & & & 3 \\
\hline 19300713 & UNLP & 011215.1 & 56.67 & 69.42 & 33 & 6.2 & 5 \\
\hline 19441121 & UNLP & 100226.2 & 56.67 & 66.28 & $33 \mathrm{R}^{*}$ & & 7 \\
\hline 19491217 & UNLP & 065328.3 & 54.24 & 69.03 & $33 \mathrm{R}^{*}$ & & 47 \\
\hline 19491217 & UNLP & 125547.7 & 54.17 & 69.02 & $33 \mathrm{R}^{*}$ & & 8 \\
\hline 19491217 & UNLP & 150753.6 & 53.89 & 69.67 & 13 & 7.5 & 55 \\
\hline 19491217 & UNLP & 223048.2 & 53.59 & 70.49 & 70 & & 5 \\
\hline 19700615 & ISC & 111454.4 & 54.31 & 64.2 & 38 & 5.7 & 285 \\
\hline
\end{tabular}

Tabla 6.1: Sismicidad histórica de la Isla de Tierra del Fuego.

\section{${ }^{*} \mathrm{R}$ : profundidad restringida}

ISS - ISC: Catálogos del International Seismological Service (Centre). Berkshire, Gran Bretaña.

UNLP: Jaschek y otros, 1982.

C, Lomn: Lomnitz, 1970

A modo de ejemplo de la importancia de la estación DSPA, se presenta el análisis de los datos correspondientes a distancias epicentrales de hasta $1000 \mathrm{~km}$ ocurridos desde junio de 1999 hasta marzo de 2002 (Plasencia y otros, 2002), Fig. 6.2. En el mismo los registros utilizados cuentan con una frecuencia de muestreo de 25 mps, las trazas fueron sometidas al control visual para determinar su integridad y calidad, se extrajeron los terremotos para su análisis preliminar y se compararon con aquellos que ya habían sido localizados por la agencia internacional IRIS (Incorporated Research Institutions for Seismology).

Para realizar la localización se usó el software SeisAn (2003) de la Universidad de Bergen, Noruega. SeisAn, para el caso de datos de una sola estación, utiliza la técnica debida a Roberts y otros (1989) que considera la polarización del primer arribo 
denominada método SML (Simplified Maximum Likelihood) y estima la magnitud utilizando la longitud de la coda en segundos.

La sismicidad local encontrada para este período corrobora las evidencias geológicas de que la región está activa. Los epicentros estimados se encuentran distribuídos sobre o cerca de las principales fallas y/o bordes de las placas.

La magnitud de los eventos procesados, determinada con la coda, no supera los 4.5 grados, hecho que explica la escasa cantidad de eventos detectados por IRIS.

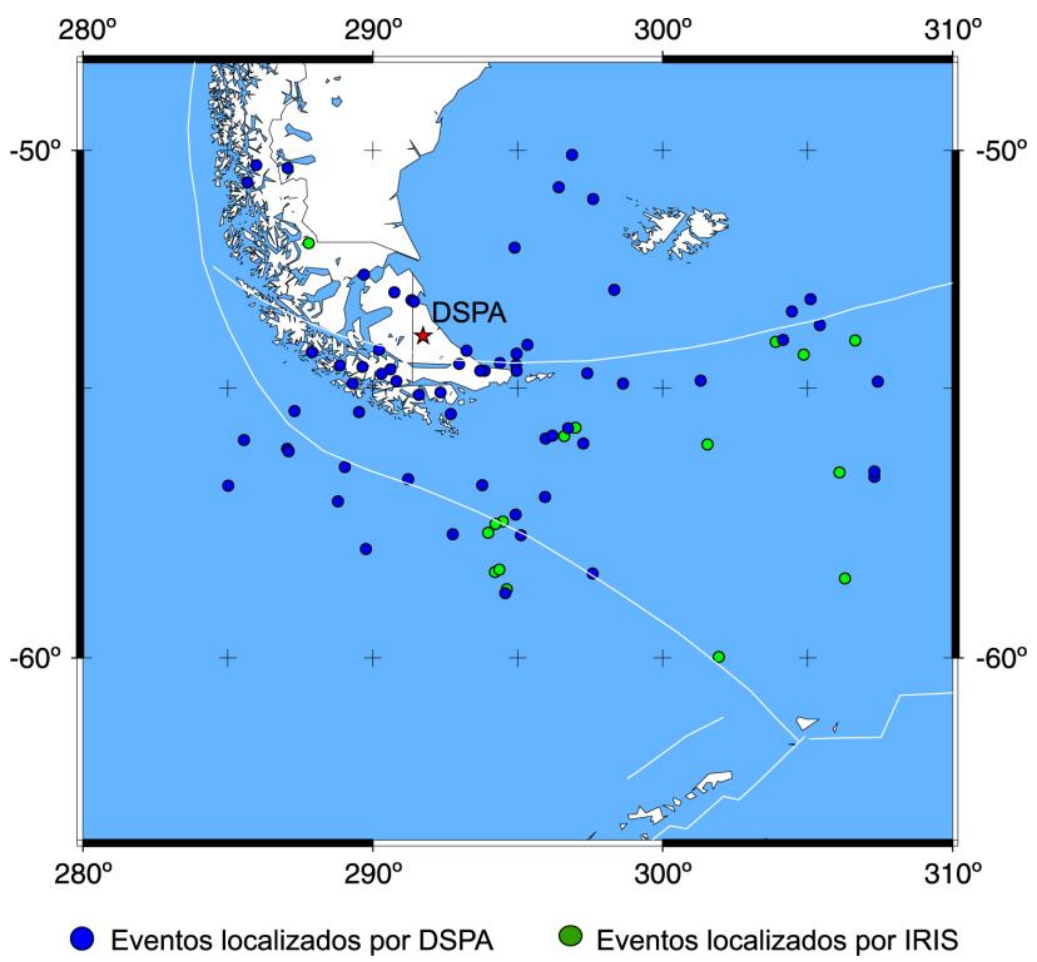

Figura 6.2: Sismos localizados con datos de DSPA durante el período 06/99-03/02 (de Plasencia y otros, 2002).

\section{2 - Estación sismológica DSPA. 1era etapa.}

\subsubsection{Instalación de instrumental de corto período}

El sensor se ubicó dentro de una caja de madera sin base, la aislación térmica se obtuvo mediante poliestireno expandido de $40 \mathrm{~mm}$. y una unidad calefactora de 2.5 W que produjo un aumento de la temperatura de aproximadamente $5^{\circ} \mathrm{C}$ en la temperatura interior a efectos de extender el rango de temperaturas externas tolerables. (Fig, 6.3). 
El adquisidor junto con el convertidor analógico digital, laptop, baterías y los paneles solares se ubicaron en un refugio a una distancia de $500 \mathrm{~m}$, vinculados a través de cable subterráneo.

Instrumental: El sensor es un LE-3Dlite Lennartz Electronic, tres canales de $1 \mathrm{~Hz}$. La adquisición se realiza a través de un adquisidor de datos de tres canales con conversor analógico digital de 16/19 bits AD7710. El tiempo se mantuvo por un receptor GPS Trimble ACE II, desde diciembre de 1999. La potencia se obtuvo a través de un panel solar $(50 \mathrm{~W})$ y baterías de $12 \mathrm{~V}$ que eran cargadas por el grupo electrógeno de la Estancia durante algunas horas de la noche, lo que aseguraba una autonomía de aproximadamente 36 horas. Además poseía un sistema calefactor con batería auxiliar.
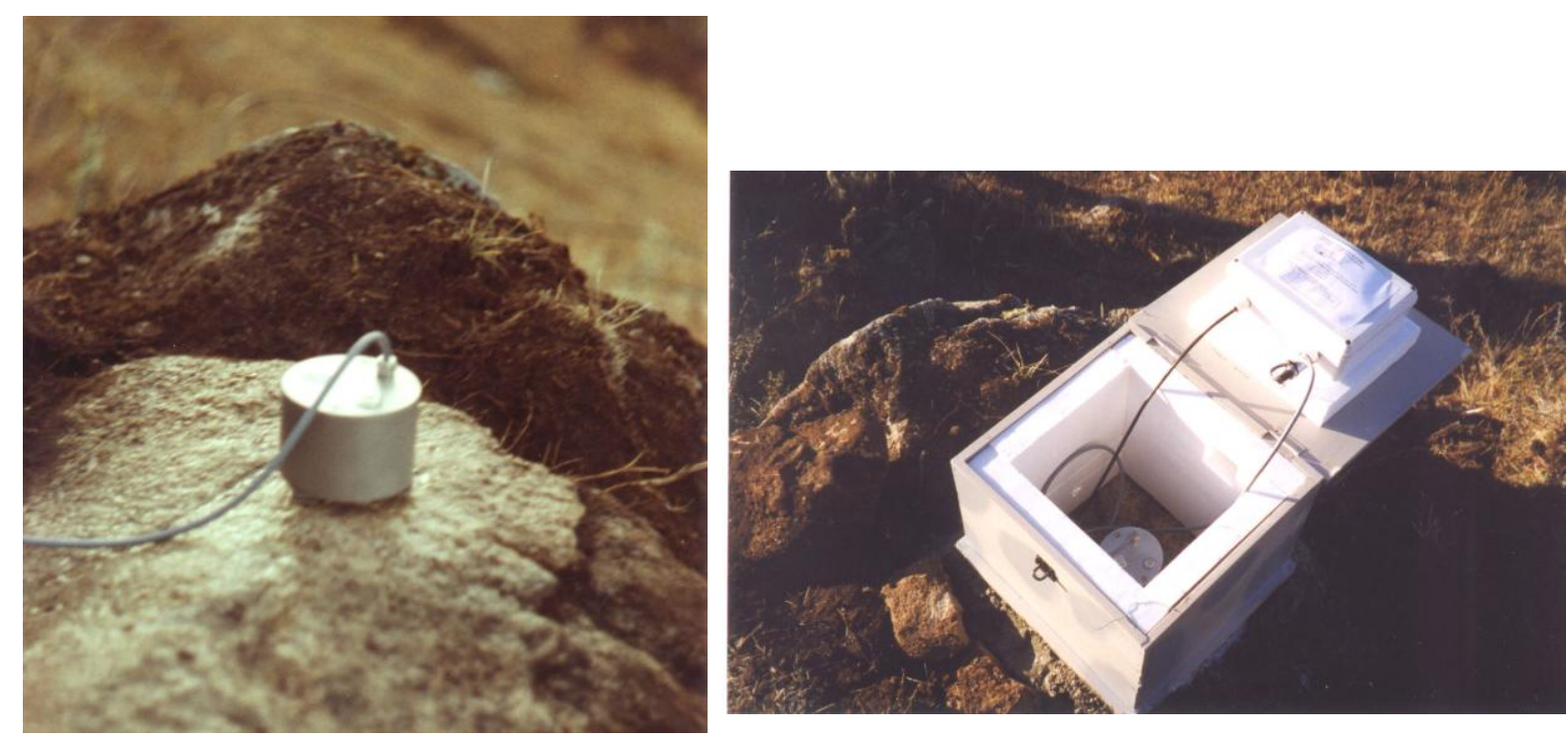

Figura 6.3: Sensor y abrigo en la Estación Despedida instalados en 1999.

Parámetros de funcionamiento:

Frecuencia de muestreo

Resolución

Registración

Sensibilidad
$25 \mathrm{sps}$, (opcional $50 \mathrm{mps}$ ).

16 bits

continua.

172 cuentas $s / \mu \mathrm{m}$

\subsection{2 - Uso de la estación}

Se muestran a modo de ejemplo dos sismogramas, Tabla 6.2, obtenidos en la Estación Río Grande, de Sabbione y otros, 2001. (Fig. 6.4 y 6.5). 
Capítulo 6: Estación Sismológica DSPA

$E^{a}$ Despedida, Tierra del Fuego, Argentina.

\begin{tabular}{|c|c|c|c|c|c|c|c|}
\hline Fecha & $\begin{array}{c}\text { Hora de } \\
\text { origen }\end{array}$ & Lat. $(\stackrel{\circ}{)}$ & Lon. $\left({ }^{\circ}\right)$ & $\begin{array}{c}\text { Prof. } \\
(\mathrm{km})\end{array}$ & $\begin{array}{c}\text { Mag. } \\
\mathrm{Mb}\end{array}$ & $\begin{array}{c}\text { Dist. Epic. } \\
\Delta^{\circ}\end{array}$ & Region \\
\hline $06 / 09 / 99$ & $04: 05: 44.5$ & $53.04 \mathrm{~S}$ & $46.84 \mathrm{~W}$ & 10 & 5.4 & $12^{\circ} 79$ & S. Atlantic Ocean \\
\hline $07 / 04 / 99$ & $06: 47: 46.2$ & $55.90 \mathrm{~S}$ & $63.40 \mathrm{~W}$ & 33 & 4.1 & $3^{\circ} 44$ & Drake Passage. \\
\hline $07 / 04 / 99$ & $06: 48: 51.9$ & $55.80 \mathrm{~S}$ & $63.00 \mathrm{~W}$ & 33 & 4.3 & $3^{\circ} 44$ & Drake Passage \\
\hline
\end{tabular}

Tabla 6.2: Parámetros focales de los eventos seleccionados.

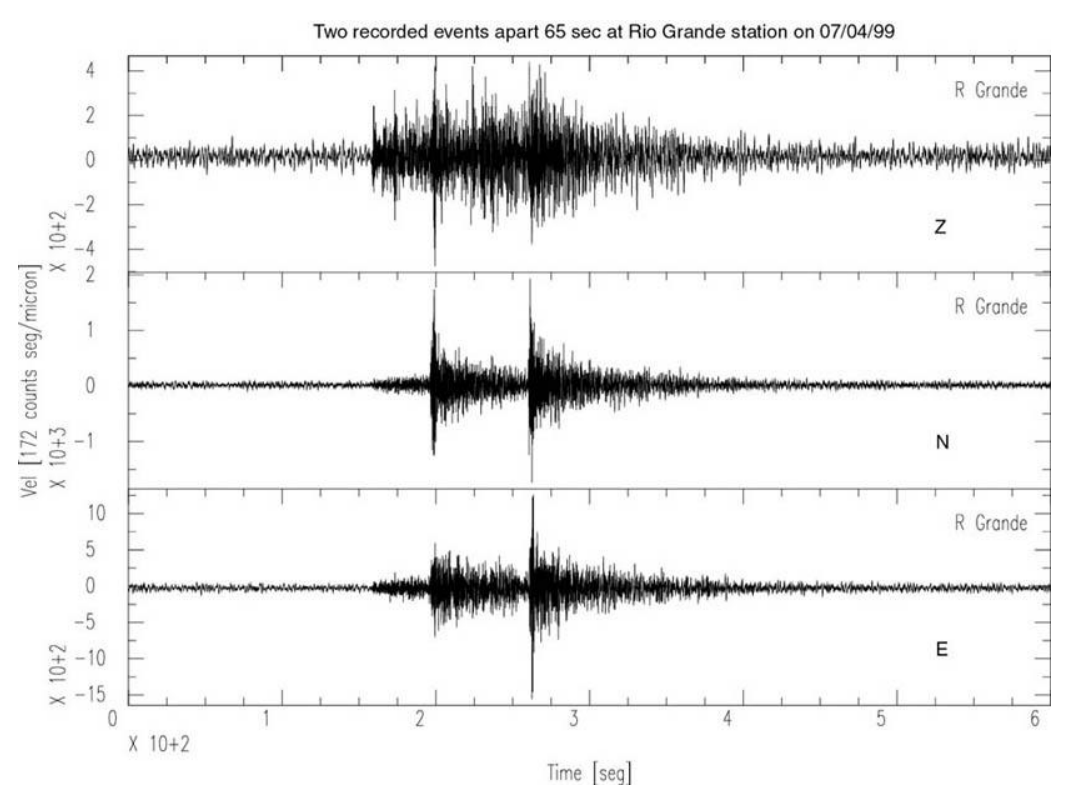

Figura 6.4: Sismograma con dos eventos registrados en la estación de Río Grande.

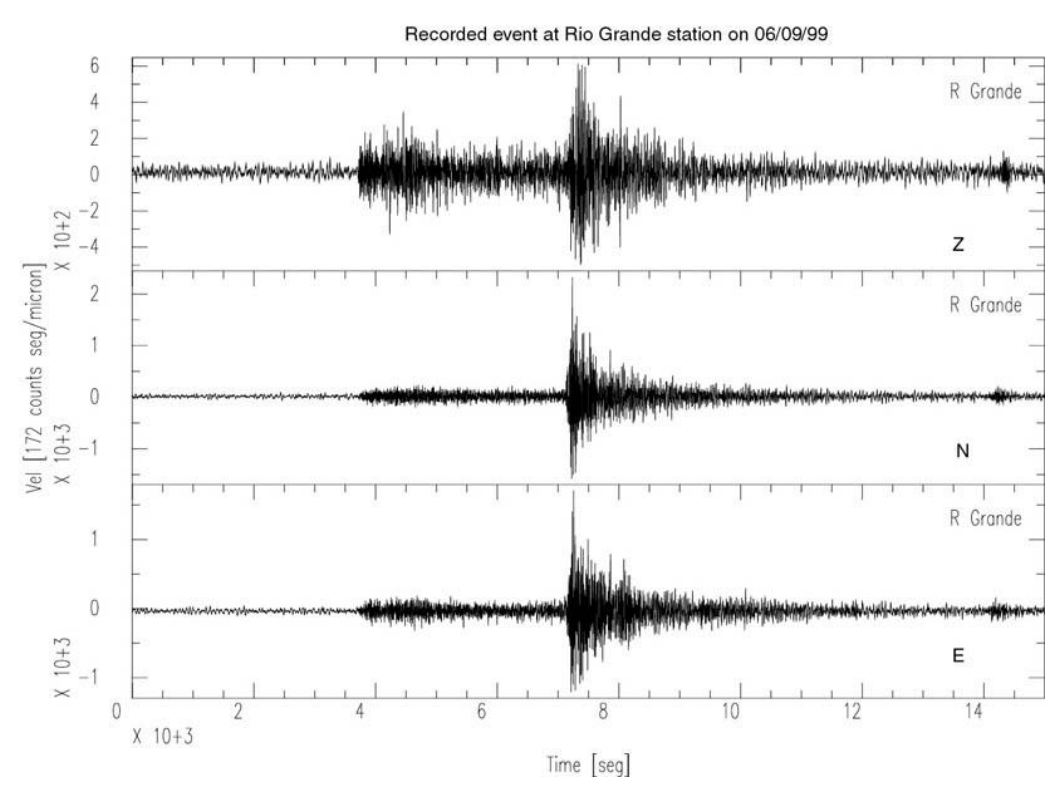

Figura 6.5: Sismograma con evento cercano a la Estación de Río Grande. 


\subsection{3 - Análisis del ruido en velocidad con instrumental de corto período}

Tierra del Fuego es una región caracterizada por bajas temperaturas y fuertes vientos. Se hizo necesario realizar un estudio de ruido sísmico para lo cual se utilizaron registros seleccionados por inspección ocular de una hora, se hizo la corrección instrumental y se correlacionaron los efectos de variables meteorológicas para el período Julio-Octubre de 1999, obtenidos en la Estación. Un espectro típico muestra un comportamiento similar para las tres componentes en esta estación, en días calmos o ventosos (Fig. 6.6). El viento produce un aumento en la banda de frecuencias estudiadas, especialmente entre $1 \mathrm{~Hz}$ y $4 \mathrm{~Hz}$, que influenciarán los registros de sismicidad local. En la Fig. 6.7 se presenta un espectro de ruido típico para la estación Despedida.

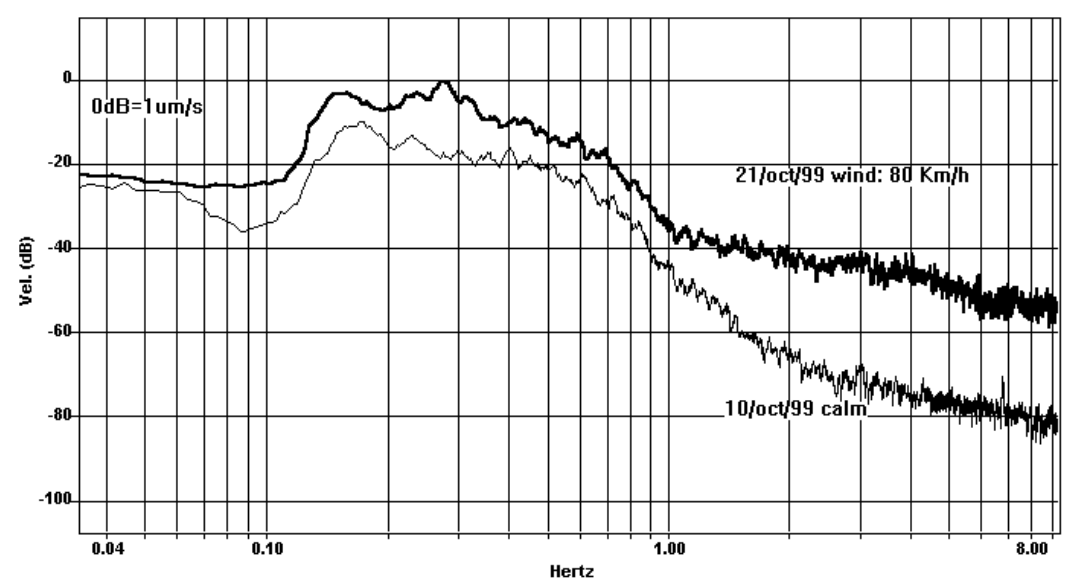

Figura 6.6: Espectro de ruido suavizado para la componente vertical en Rio Grande, luego de efectuada la corrección instrumental: en línea remarcada se presenta un día ventoso ( $36 \mathrm{~km} / \mathrm{h}$ con ráfagas de 80 $\mathrm{km} / \mathrm{h}$ ); en línea fina se muestra el nivel de ruido para un día calmo (velocidad media del viento de 5,4 km/h y ráfagas de $17,7 \mathrm{~km} / \mathrm{h}$ )

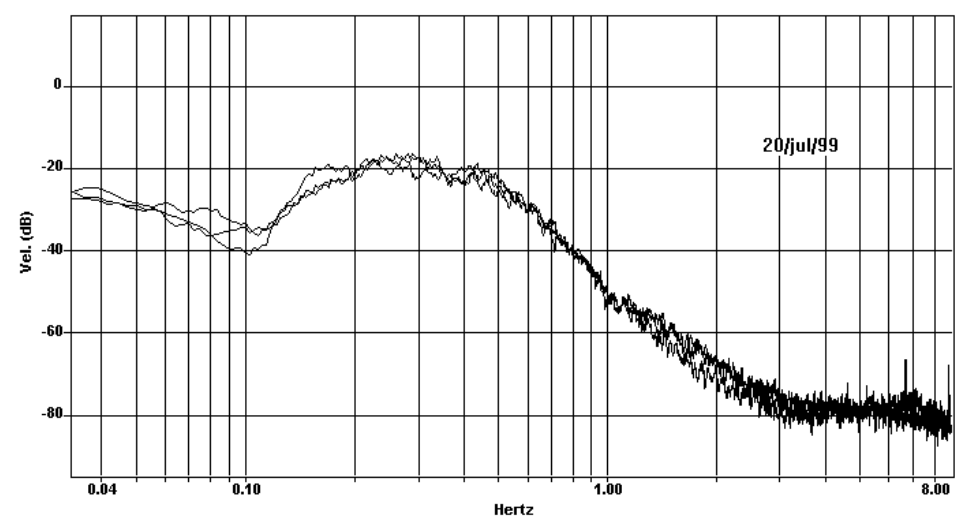

Figura 6.7:

Hert

típico en Despedida 


\subsection{Estación sismológica DSPA. 2da etapa.}

\subsubsection{Instalación de instrumental banda ancha}

La puesta en funcionamiento del nuevo instrumental, que comprende también un teléfono satelital para la recuperación en forma remota de los datos, se efectuó entre el 24 y el 29 de noviembre de 2002, fecha en la cual el nuevo sismógrafo ha comenzado a funcionar.

El instrumental consiste en un sismómetro Guralp Digital CMG 3T, unidad de disco SAM, computadora personal HP, baterías OPTIMA D1000S Yellow TOP 60 Ah $12 \mathrm{~V}$ y un sistema de potencia basado en paneles solares (2 paneles de 60Ah cada uno). Las curvas de respuesta en frecuencia para este instrumental se muestra en la Fig. 6.8.
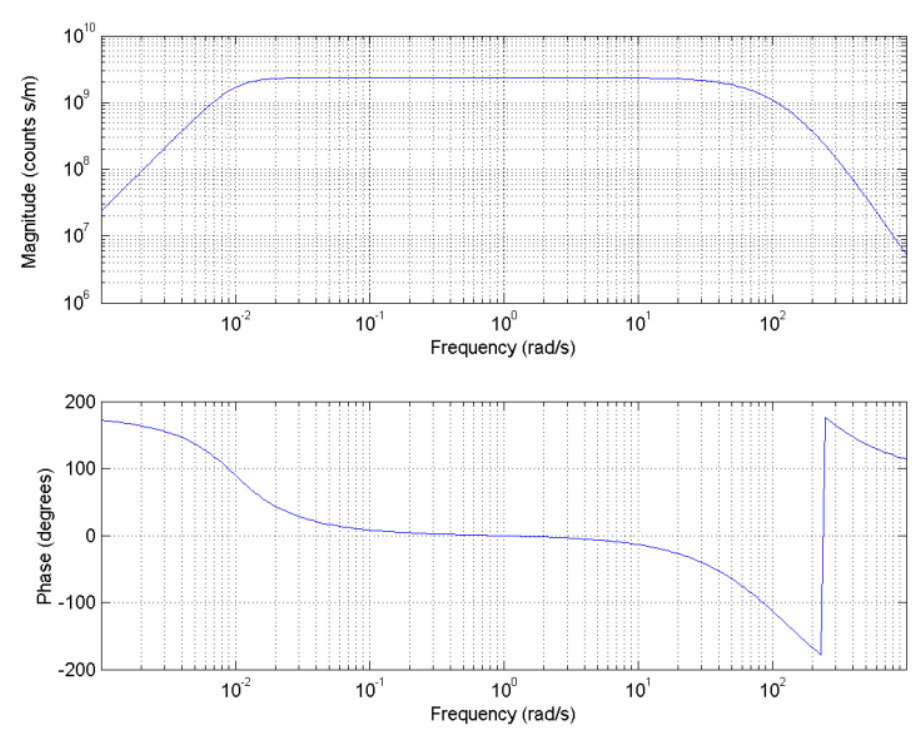

Figura 6.8: Curvas de respuesta en frecuencia correspondientes a la estación DSPA,

El sitio donde se ha ubicado el sensor es el mismo en el cual se encontraba funcionando el sensor de corto período desde el año 1999, habida cuenta de los estudios previos realizados del ruido que demuestran su bajo nivel (Fig. 6.9).

El personal de la Estación Astronómica de Río Grande (EARG) es el responsable de su funcionamiento. 

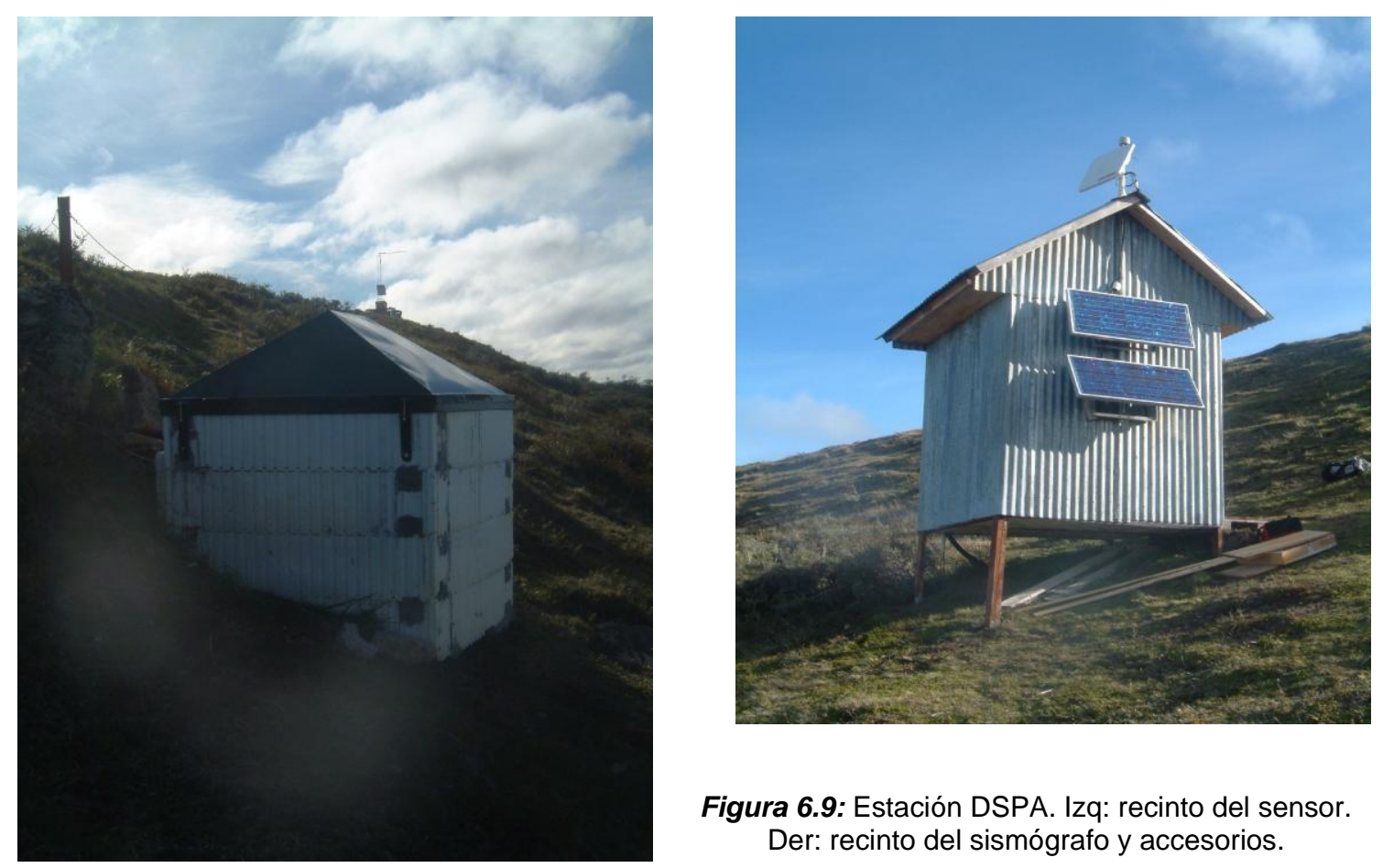

Figura 6.9: Estación DSPA. Izq: recinto del sensor. Der: recinto del sismógrafo y accesorios.

\subsubsection{Uso de la estación}

Se presenta a modo de ejemplo (Fig. 6.10) el registro obtenido en la estación DSPA correspondiente al evento que se detalla en la Tabla 6.3, con datos extraídos del USGS.

Este terremoto ocurrió en el límite entre la placa de Scotia y la placa Antártica. En la región epicentral la placa de Scotia se está moviendo hacia el oeste-noroeste con respecto a la placa Antártica. La velocidad relativa entre las dos placas es de aproximadamente 1cm/año (NEIC-USGS). En este límite se observan mayoritariamente fallas transformantes, que involucran predominantemente fallas de dirección, tal es el caso de este evento, aunque en esta región también han ocurrido terremotos asociados a fallas normales.

\begin{tabular}{|c|c|c|c|c|c|c|c|}
\hline Fecha & $\begin{array}{c}\text { Hora de } \\
\text { origen }\end{array}$ & Lat. $\left(^{\circ}\right)$ & Lon. $\left(^{\circ}\right)$ & $\begin{array}{c}\text { Prof. } \\
(\mathrm{km})\end{array}$ & $\begin{array}{c}\text { Mag. } \\
\text { Mw }\end{array}$ & $\begin{array}{c}\text { Dist. Epic. } \\
\Delta^{\circ}\end{array}$ & Region \\
\hline $04 / 08 / 03$ & $04: 37: 20.1$ & $60.53 \mathrm{~S}$ & $43.41 \mathrm{~W}$ & 10 & 7.6 & $14^{\circ} 92$ & Scotia Sea \\
\hline
\end{tabular}

Tabla 6.3: Parámetros focales del terremoto del 4 de agosto de 2003. 


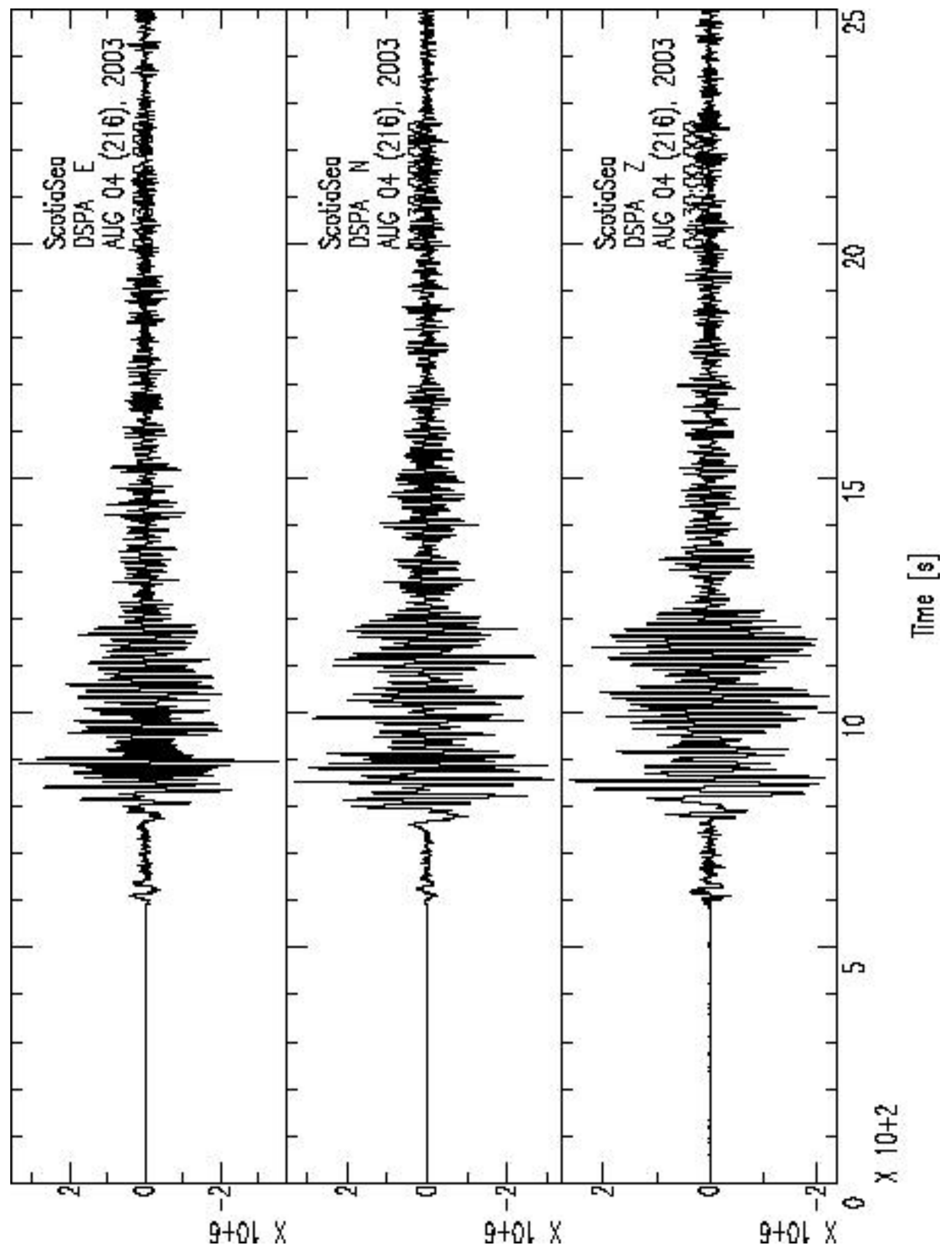

Figura 6.10: ejemplo de sismograma superficial de gran magnitud registrado en la estación DSPA. 


\section{APLICACIONES}




\section{Capítulo 7}

\section{DETERMINACIÓN E INVERSION DE CURVAS DE DISPERSION DE ONDAS}

RAYLEIGH EN DISTINTAS TRAYECTORIAS HACIA LA PLATA (PCIA. de BS. AS.)

\section{1 - Introducción}

La región andina representa un margen continental activo entre la placa de Nazca y la placa continental Sudamericana. Una característica de este proceso es la variación norte-sur del ángulo de subducción, que alterna subducción normal y plana (Jordan y otros, 1983; Cahill e Isacks, 1992).

Las Sierras Pampeanas constituyen la provincia más oriental de la orogenia andina y son bloques de grandes cadenas de basamento cristalino y anchos valles que se extienden norte-sur. Penetran por el norte en la Puna Argentina (parte sur del Altiplano), la cordillera oriental y la zona sur del cinturón subandino. Hacia el este y el sur, limitan con rocas cratónicas no deformadas (Fig. 7.1).

En particular, entre los $27^{\circ}$ y los $32^{\circ} S$, la placa de Nazca subducta horizontalmente. Esta región está caracterizada por cinturones que corren paralelos a la costa Pacífica, siendo las principales unidades morfológicas, la Cordillera Frontal Los Andes-, la Precordillera, y las Sierras Pampeanas, amplia zona de basamento elevado, consideradas como una característica tectónica distinguible en regiones de subducción plana (Smalley y otros,1993).

Es sabido que el espesor de la corteza varía con el ángulo de subducción. Es muy profunda bajo el Altiplano-Puna. La mayor cantidad de determinaciones de espesores corticales proviene de estudios sísmicos (Zandt y otros, 1994; Beck y otros, 1996) y también de datos gravimétricos y magnetotelúricos (Götze y otros, 1994; Schwartz y otros, 1994). Los datos disponibles indican que en el Altiplano el espesor cortical es de los mayores del mundo y que puede exceder los $70 \mathrm{~km}$ (Dorbath y otros, 1993).

Para latitudes correspondientes a la Puna Argentina, el espesor cortical es de aproximadamente $65 \mathrm{~km}$ y disminuye a $52-57 \mathrm{~km}$ debajo de la Precordillera y Sierras Pampeanas, sugiriendo un incremento en la inclinación hacia el oeste (James y Snoke, 1994; Whitman,1994).

Snoke y James (1997) determinaron la profundidad promedio del Moho en las cuencas del Paraná y Chaco, ubicadas al este de la Puna argentina (Fig. 7.1). Encontraron que el espesor cortical de la cuenca del Chaco es de sólo $32 \mathrm{~km}$, con modelos de velocidad suavemente menores que en la cuenca del Paraná. 
En latitudes correspondientes a la región de las Sierras Pampeanas, se hicieron distintos estudios geofísicos para determinar el espesor de la corteza con datos sismológicos, gravimétricos y magnetotelúricos (Reigner y otros, 1992; Smalley y otros, 1993; Whitman, 1994; Pomposiello y otros, 1994,1998, 2002; Rosello y otros, 1996). Reigner y otros (1994) estimaron la variación del espesor cortical usando ondas convertidas. Estos autores encontraron que bajo las Sierras Pampeanas, el Moho está a una profundidad cercana a los $52 \mathrm{~km}$, debajo de la precordillera oriental se inclina hasta los 55-57 km y debajo de Los Andes, hasta $60 \mathrm{~km}$

Mooney y otros (1998) presentaron un modelo global de la corteza terrestre con una resolución de $5^{\circ} \times 5^{\circ}$, CRUST 5.1, para distintas regiones geológicas, tales como escudos precámbricos, márgenes continentales, orogenias, etc. Una de las aplicaciones es que con este modelo se pueden predecir estructuras corticales en regiones donde, hasta ahora, no hay mediciones sísmicas disponibles, tal como ocurre en una gran parte de América del Sur.

En particular, no hay demasiados estudios previos en la zona andina sur incluyendo las Sierras Pampeanas debido a la escasa instrumentación sismológica permanente. Se decidió entonces utilizar los registros obtenidos en LPA para determinar la velocidad de propagación en la región de las Sierras Pampeanas y a partir de ésta, el espesor cortical. Aunque no se pueden obtener las variaciones laterales de la densidad de la corteza usando solamente una estación de registro, se consigue obtener un valor promedio de su espesor. Este resultado puede ser comparado y correlacionado con los valores procedentes de los modelos globales.

En un estudio previo (Sabbione et al., 1997) se obtuvieron curvas de dispersión para distintas trayectorias en el continente sudamericano a partir de datos de LPA, que verificaron la calidad y resolución de los mismos para encarar este tipo de estudio.

Con este resultado, y teniendo en cuenta el objetivo propuesto, se seleccionaron eventos tales que en su trayectoria hacia la estación atravesaran el sistema de Sierras Pampeanas, a fin de caracterizar sus respuestas. Se analizaron los sismogramas usando la Técnica de Filtrado Múltiple (Dziewonski y otros, 1969; Levshin y otros, 1972) para obtener las curvas de dispersión. Luego se invirtieron estas curvas aplicando el programa Udima (D. Lokshtanov, 1993) para obtener el modelo de velocidad de las diferentes trayectorias, utilizando como entrada para el proceso de inversión, los modelos estimados por CRUST 5.1. 
Finalmente, se compararon los resultados obtenidos a partir de los datos sismológicos con los obtenidos previamente a partir de datos magnetotelúricos de la zona de las Sierras Pampeanas.

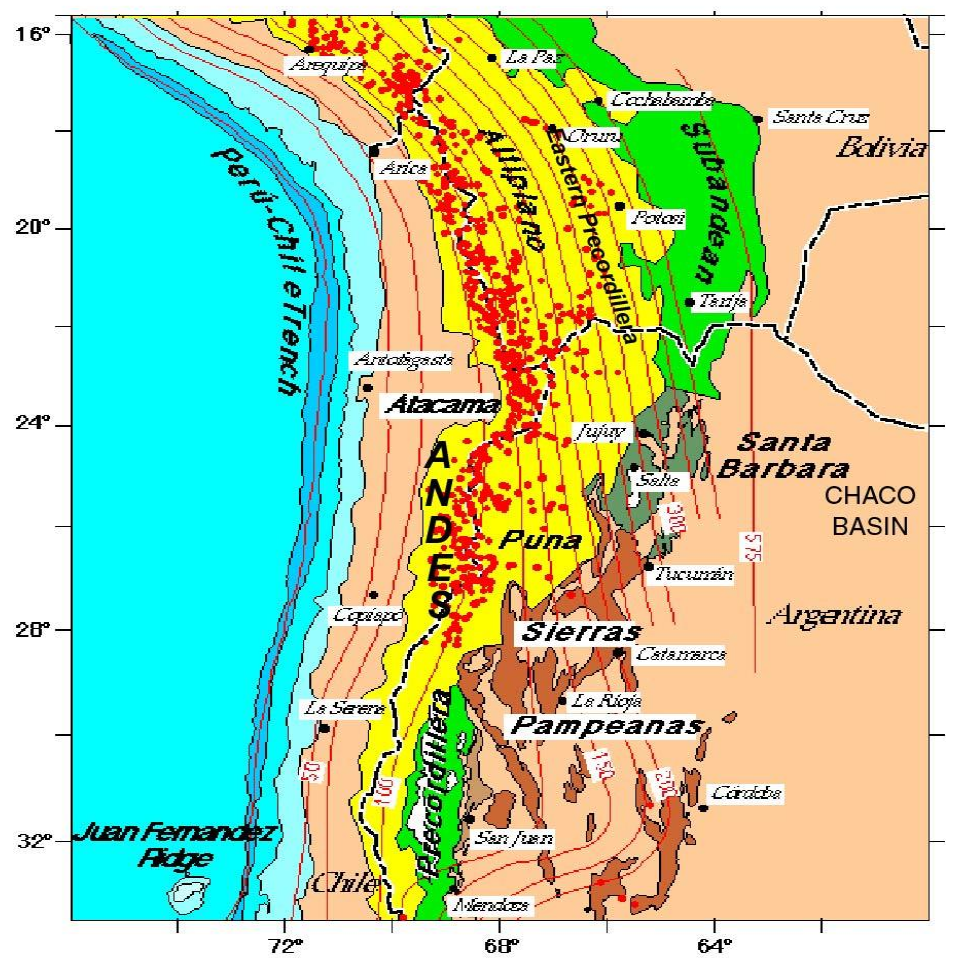

Figura 7.1: Rasgos tectónicos básicos de la zona de estudio. Las líneas llenas indican la curvas de nivel de la zona de Wadati-Benioff, los puntos indican la distribución de los volcanes jóvenes, $(<6 \mathrm{Ma})$ (de Cahill e Isacks, 1992).

Se completó el estudio analizando eventos cuyas trayectorias no incluyeran las Sierras Pampeanas: dos grupos, incluyendo eventos que proceden del norte y del oeste, respectivamente, atravesando solamente áreas cratónicas y otro grupo proveniente del oeste que cruza la cadena de Los Andes.

\subsection{Estudio previo}

Como antecedente, se puede mencionar un estudio previo (Sabbione et al, 1997), donde se utilizaron eventos provenientes de distintos focos en Chile, Perú, Ecuador y Bolivia, registrados en la estación digital de La Plata (frecuencia de

\begin{tabular}{||c|c|c|c|c|c|c|c||}
\hline \hline & $\begin{array}{c}\text { Fecha } \\
\mathrm{d} \mathrm{m} \mathrm{a}\end{array}$ & $\begin{array}{c}\text { Hora de } \\
\text { origen } \\
\mathrm{h} \mathrm{m} \mathrm{s}\end{array}$ & $\begin{array}{c}\text { Coordenadas } \\
\varphi^{\circ} \mathrm{S} \lambda^{\circ} \mathrm{W}\end{array}$ & $\begin{array}{c}\text { Prof. } \\
\mathrm{km} .\end{array}$ & $\begin{array}{c}\text { Magnitud } \\
\mathrm{mb}\end{array}$ & $\begin{array}{c}\text { Distancia } \\
\text { epicentral } \\
\Delta(\mathrm{km})\end{array}$ & $\begin{array}{c}\text { Azimut } \\
\text { back-Az }\end{array}$ \\
\hline
\end{tabular}



en distintas trayectorias hacia La Plata (Pcia. de Bs. As.).

\begin{tabular}{|c|c|c|c|c|c|c|c|}
\hline & $\begin{array}{l}\text { Fecha } \\
\mathrm{d} \mathrm{m} \mathrm{a}\end{array}$ & $\begin{array}{l}\text { Hora de } \\
\text { origen } \\
\mathrm{h} \mathrm{m} \mathrm{s}\end{array}$ & $\begin{array}{l}\text { Coordenadas } \\
\varphi^{\circ} S \quad \lambda^{\circ} \mathrm{W}\end{array}$ & $\begin{array}{l}\text { Prof. } \\
\mathrm{km} .\end{array}$ & $\begin{array}{l}\text { Magnitud } \\
\mathrm{mb}\end{array}$ & $\begin{array}{c}\text { Distancia } \\
\text { epicentral } \\
\Delta(\mathrm{km})\end{array}$ & $\begin{array}{c}\text { Azimut } \\
\text { back-A } z^{\circ}\end{array}$ \\
\hline 1 & $11-06-95$ & $02: 18: 43.6$ & $19.067 \quad 68.344$ & 59 & 5.2 & 2.031 & 327 \\
\hline 2 & $10-03-95$ & $01: 51: 23.9$ & $\begin{array}{ll}2.750 & 77.881\end{array}$ & 24 & 6.5 & 4.108 & 325 \\
\hline 3 & $07-30-95$ & $10: 35: 39.2$ & $\begin{array}{ll}24.359 & 70.715\end{array}$ & 11 & 5.8 & 1.697 & 310 \\
\hline 4 & $04-19-96$ & $00: 19: 31.1$ & $23.944 \quad 70.093$ & 50 & 6.0 & 1.688 & 313 \\
\hline 5 & $02-22-96$ & $13: 40: 53.5$ & $\begin{array}{ll}33.693 & 71.706\end{array}$ & 44 & 5.9 & 1.273 & 272 \\
\hline 6 & $09-09-96$ & $00: 20: 39.1$ & 71.560 & 39 & 6.0 & 1.308 & 281 \\
\hline 7 & $04-06-96$ & $12: 17: 29.3$ & $53.449 \quad 76.351$ & 10 & 5.1 & 2.515 & 209 \\
\hline 8 & $02-19-96$ & $07: 10: 10.3$ & 42.091 & 33 & 5.9 & 1.689 & 237 \\
\hline
\end{tabular}

Tabla 7.1. Eventos seleccionados con sus parámetros focales. (de Sabbione y otros, 1997).

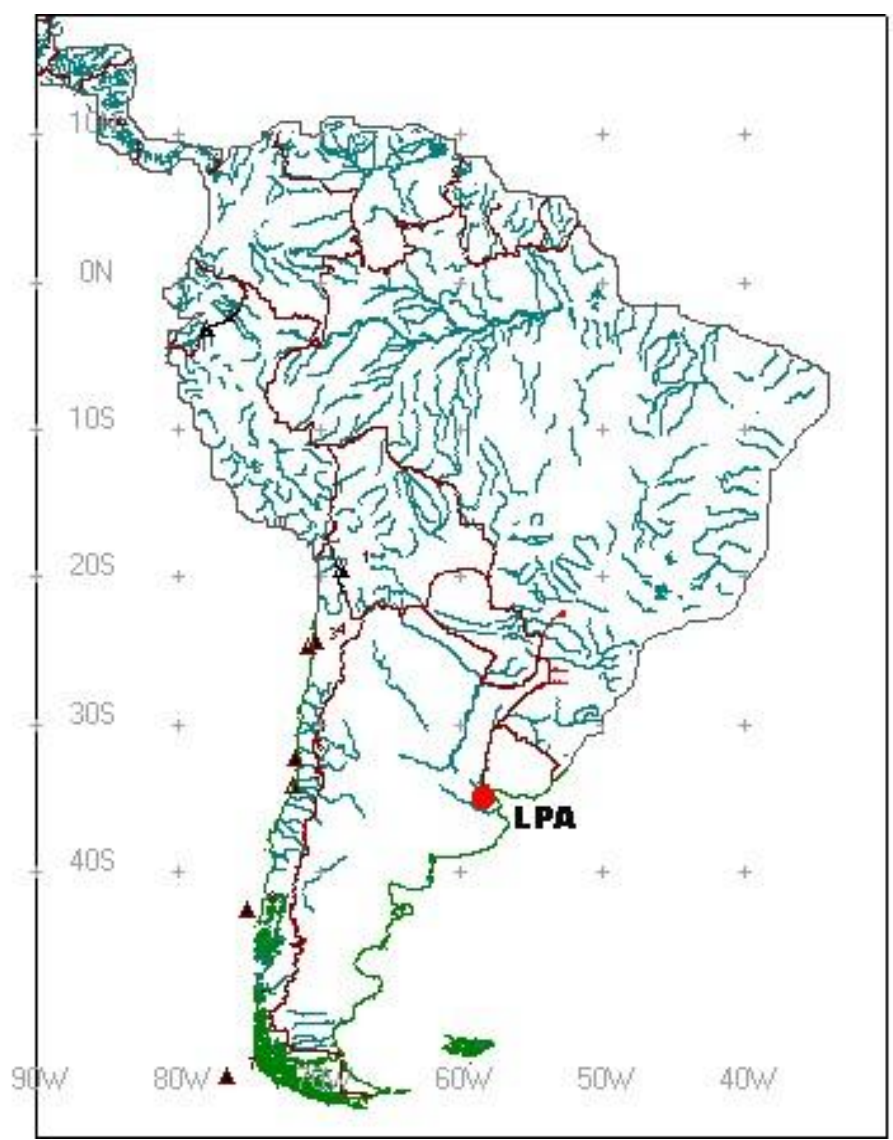

Figura 7.2. Mapa del continente sudamericano, donde se grafican los eventos considerados y la estación sismológica LPA. (de Sabbione y otros, 1997).

muestreo: $20.14 \mathrm{~Hz}$ ), con trayectorias hasta la estación casi en su totalidad continentales. Los eventos seleccionados figuran en la tabla 7.1, a través de sus parámetros focales, distancia epicentral y el azimut desde La Plata (back-azimut). 
En la Fig. 7.2 se muestra la ubicación espacial de los eventos mencionados, con triángulos y de la estación sismológica LPA con un círculo. En la misma se puede apreciar que, a los efectos de analizar las distintas trayectorias, es factible dividir los ocho eventos, tomados de a dos, en cuatro zonas, según sus azimuts desde La Plata. De esta manera se consideran los back-azimuts promedio de $326^{\circ}$ (zona 1), $311^{\circ}$ (zona 2), 276 (zona 3) y 223ำ (zona 4) respectivamente.

El cálculo de las curvas de dispersión se realizó con la técnica de Filtrado Múltiple (Dziewonski et al. 1969), de ventana móvil, que estima el espectro de la señal en una pequeña ventana que se desplaza a lo largo de la misma. Esta técnica supone que dentro de la misma, la señal puede ser considerada estacionaria.

Los datos preparados entran al programa que primeramente calcula la Transformada de Fourier de la señal, y selecciona las frecuencias centrales entre un $T_{i}(s)$ y un $T_{f}(s)$. Para cada una de estas frecuencias centrales construye un filtro Gaussiano de banda proporcional a la frecuencia central y resolución controlada por el parámetro $\alpha$, que en este caso tomó el valor de 50 .

Para cada período útil se multiplica el espectro de la señal por el filtro y se antitransforma, obteniéndose la señal filtrada en el dominio del tiempo; luego se reitera este proceso para todos los períodos útiles. Se calculan las envolventes normalizadas como funciones del tiempo (o velocidad) y del período. Como el instante donde se produce el máximo de la envolvente corresponde al tiempo en el que el paquete de ondas se desplaza con la velocidad de grupo $U=U(T)$, se trazan curvas de nivel y luego, graficando los máximos, se obtienen las curvas de velocidad versus período para las distintas trayectorias seleccionadas.

Aplicando este método se obtuvieron las curvas de dispersión de ondas Rayleigh para diversas trayectorias, casi en su totalidad continentales, hacia La Plata. En la Fig. 7.3 se muestran las curvas resultantes discriminadas según las zonas ya descriptas:

* En las zonas 1 y 2, trayectorias que provienen desde el noroeste de nuestra estación, se pueden distinguir claramente el modo fundamental y el modo superior de las ondas Rayleigh.

* En la zona 3, trayectorias que provienen prácticamente del oeste de nuestra estación, hay una clara curva dispersiva del modo fundamental.

* En la zona 4, trayectorias que provienen del sudoeste de nuestra estación, hay también una clara coincidencia en las curvas de dispersión en el modo fundamental. 
* Observando ahora la pendiente de las curvas obtenidas en esta primera etapa del análisis de ondas superficiales, se puede apreciar que el comportamiento dispersivo en las trayectorias consideradas no es homogéneo.

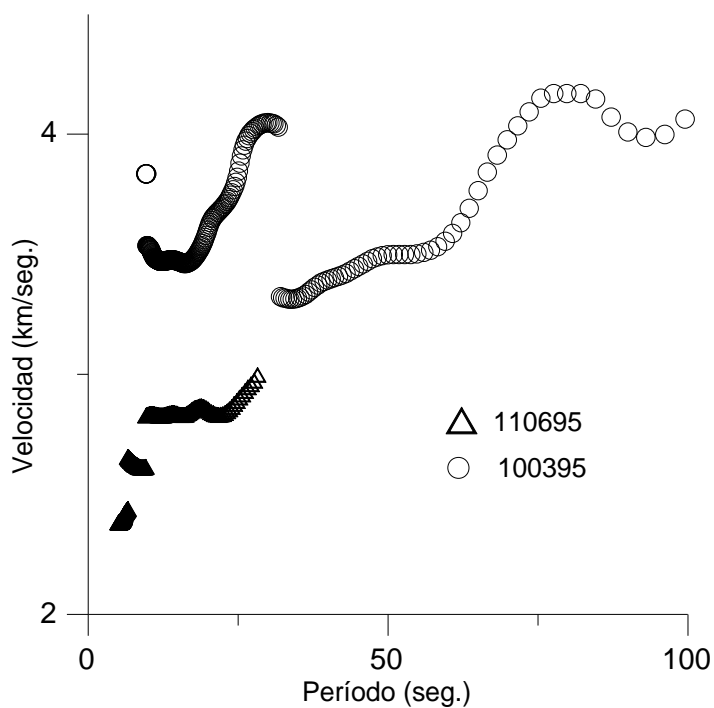

Zona 1. BackAz. promedio $326^{\circ}$.

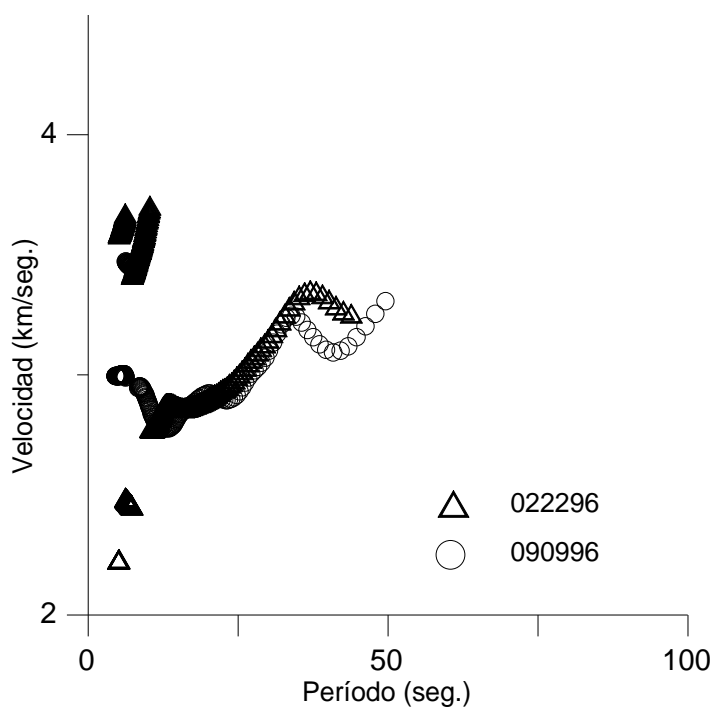

Zona 3. BackAz. promedio $276^{\circ}$.

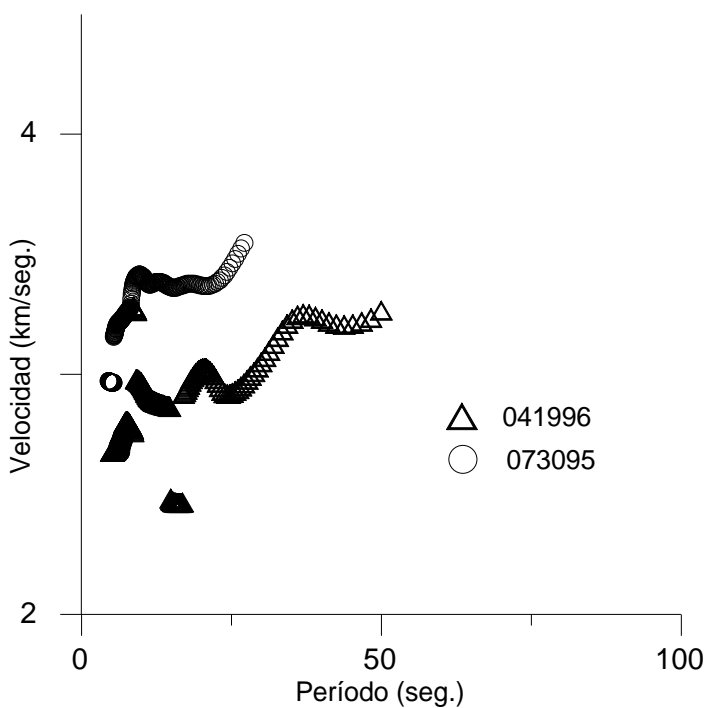

Zona 2. BackAz. promedio $311^{\circ}$.

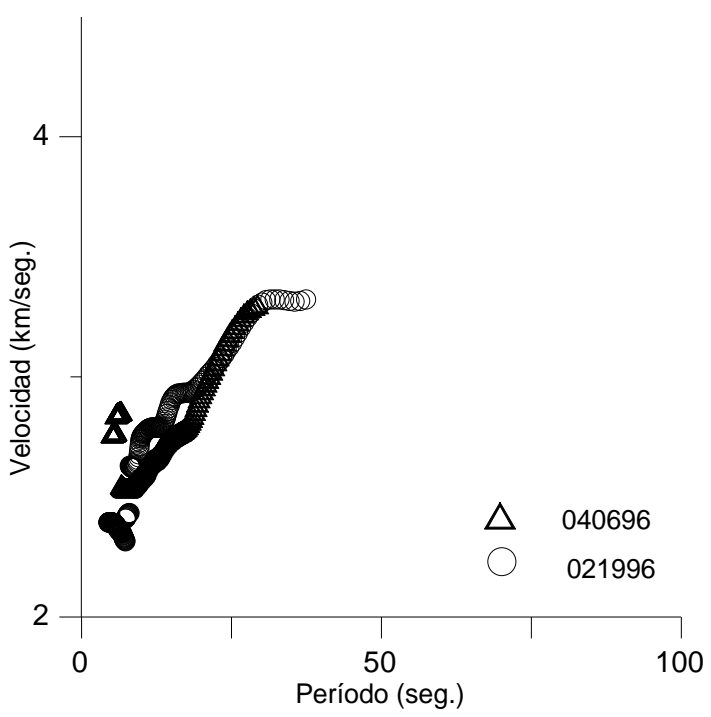

Zona 4. BackAz. promedio: $223^{\circ}$

Figura 7.3. Curvas de dispersión de las cuatro zonas consideradas.

A través de este estudio se ha podido comprobar que los datos de LPA resultan útiles para obtener curvas de dispersión que reflejan claramente las distintas trayectorias entre evento y estación. Esto justifica, en una segunda etapa trabajar con 
datos de esta estación, realizar su inversión y obtener perfiles de velocidad de corteza y manto superior. A este efecto, se decidió estudiar en particular la región de las Sierras Pampeanas, ya que de la misma se cuenta con otros datos geofísicos que permiten realizar un análisis de nuestros resultados y además se trabaja con datos de otras zonas como control del procesamiento.

\subsection{Datos}

Para poder estudiar en particular la región de las Sierras Pampeanas usando datos de la estación LPA, fue necesario utilizar eventos que ocurrieron al oeste de esta formación. La selección efectuada estuvo condicionada por la sismicidad y por las características de nuestra estación sismológica. Luego, para comparar entre esas trayectorias y otras continentales, se eligieron eventos que no incluyeran las Sierras Pampeanas en su trayectoria hasta la estación. Finalmente se utilizó una estación receptora alternativa, CPUP (Villa Florida, Paraguay) en eventos que en su trayectoria recorrieran la región de estudio, a efectos de realizar una comparación con los resultados obtenidos (Sabbione y otros, 2003).

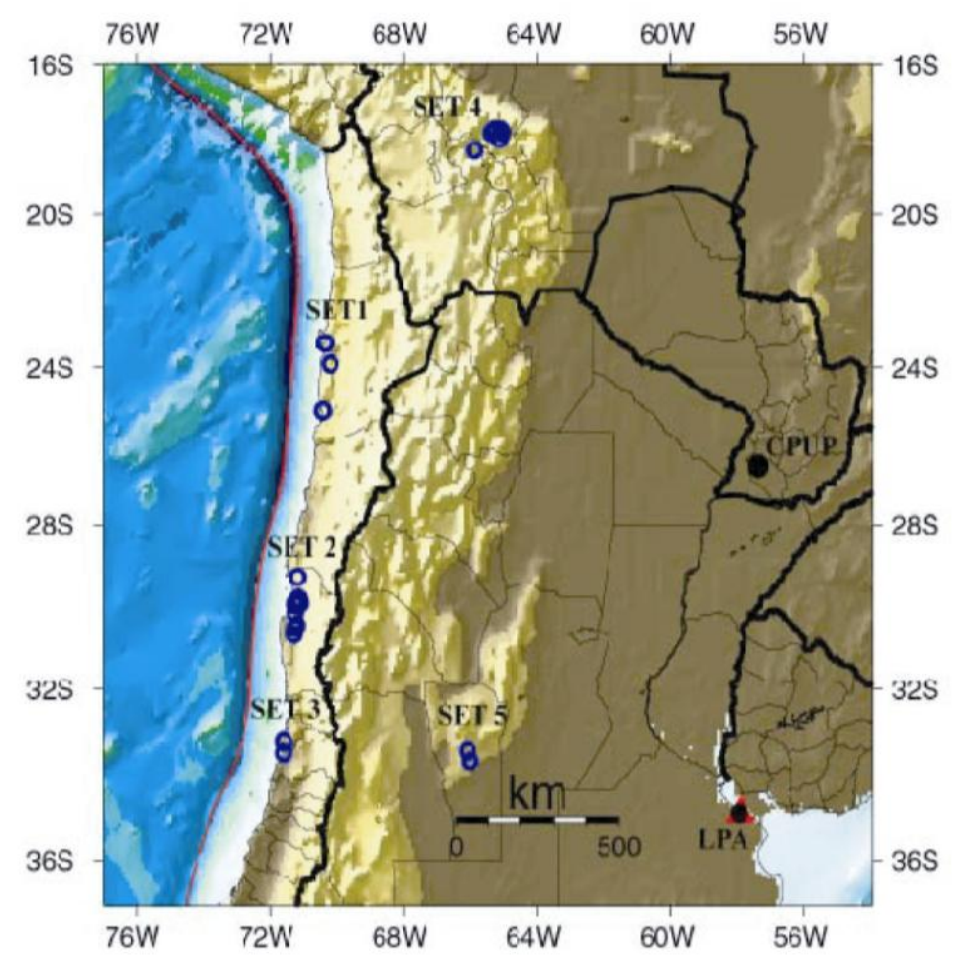

Figura 7.4. Mapa que muestra la ubicación de los epicentros correspondientes a los diferentes Grupos (1 a 5), además de las estaciones sismológicas LPA y CPUP (de Sabbione et al, 2003). 
La ubicación geográfica de los eventos seleccionados nos permite la formación de cinco grupos (Fig. 7.4).

Set 1 incluye eventos con latitudes entre $23^{\circ}$ y $25^{\circ} \mathrm{S}$ y longitud $70^{\circ} \mathrm{W}$, cuyas trayectorias atraviesan Los Andes, las Sierras Pampeanas ( $27^{\circ}$ y $29^{\circ} \mathrm{S}$ y $64^{\circ}$ y $69^{\circ} \mathrm{W}$ ) y una zona cratónica, con distancias epicentrales a La Plata de $1700 \mathrm{~km}$.

Set 2 incluye eventos con epicentros cercanos a $30^{\circ} \mathrm{S}$ y $72^{\circ} \mathrm{W}$, que atraviesan trayectorias similares con distancias de $1400 \mathrm{~km}$.

Set 3 incluye eventos localizados cerca de $33^{\circ} \mathrm{S}$ y $72^{\circ} \mathrm{W}$, cruzando Los Andes y una región cratónica, con distancias de $1300 \mathrm{~km}$.

Set 4 corresponde a eventos con epicentros cercanos a $18^{\circ} \mathrm{S}$ y $65^{\circ} \mathrm{W}$, con trayectorias que atraviesan áreas cratónicas, y con distancias a la estación de cerca de $2000 \mathrm{~km}$.

Set 5 incluye eventos localizados cerca de $33.5^{\circ} \mathrm{S}$ y $66^{\circ} \mathrm{W}$, donde también las trayectorias cruzan áreas cratónicas y las distancias son de 750 km.

\section{4 - Procesamiento}

Se aplicó un preproceso a los datos mediante el programa PITSA (Scherbaum y Johnson, 1993). El mismo incluye la aplicación de la corrección por instrumento y la corrección por línea base, a efectos de determinar y sustraer las componentes de variación lenta. Luego, las trazas se filtraron con un pasabandas Butterworth entre las frecuencias $0.014 \mathrm{~Hz}$ y $1 \mathrm{~Hz}$ (1 seg y $70 \mathrm{seg}$ ), en algunos casos y $0.014 \mathrm{~Hz}$ y $0.1 \mathrm{~Hz}$ (10 seg y $70 \mathrm{seg}$ ) en otros, para disminuir la componente de ruido presente. Dicho filtro es del tipo recursivo en el dominio del tiempo y utiliza la transformada $Z$ bilineal. Por último se llevaron todas las trazas a $2 \mathrm{~Hz}$ disminuyendo así la frecuencia de digitalización.

El análisis espectral de los datos se realiza usando el programa de cálculo F.T.A.N. (Frequency-Time Analysis, Levshin y otros, 1972). El mismo está basado en la técnica de Filtrado Múltiple (Kocaoglu y Long, 1993) y permite obtener las curvas de dispersión. Este programa requiere un encabezamiento para cada traza. Para confeccionar este archivo que contiene la información del evento y la estación se utilizó el programa SAC (Tapley, 2000). 
Capítulo 7: Determinación e Inversión de curvas de dispersión de ondas Rayleigh en distintas trayectorias hacia La Plata (Pcia. de Bs. As.).

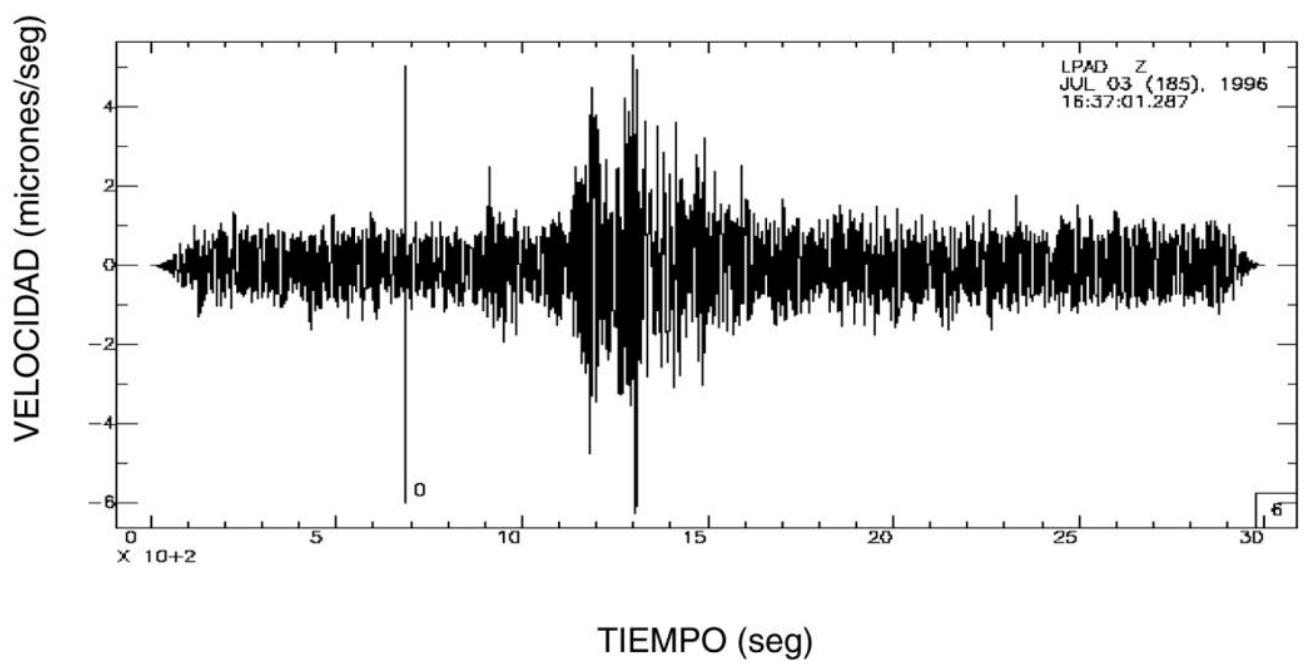

Figura 7.5. Evento del Set 1 de fecha 3 de julio de 1996 registrado en la estación LPA.

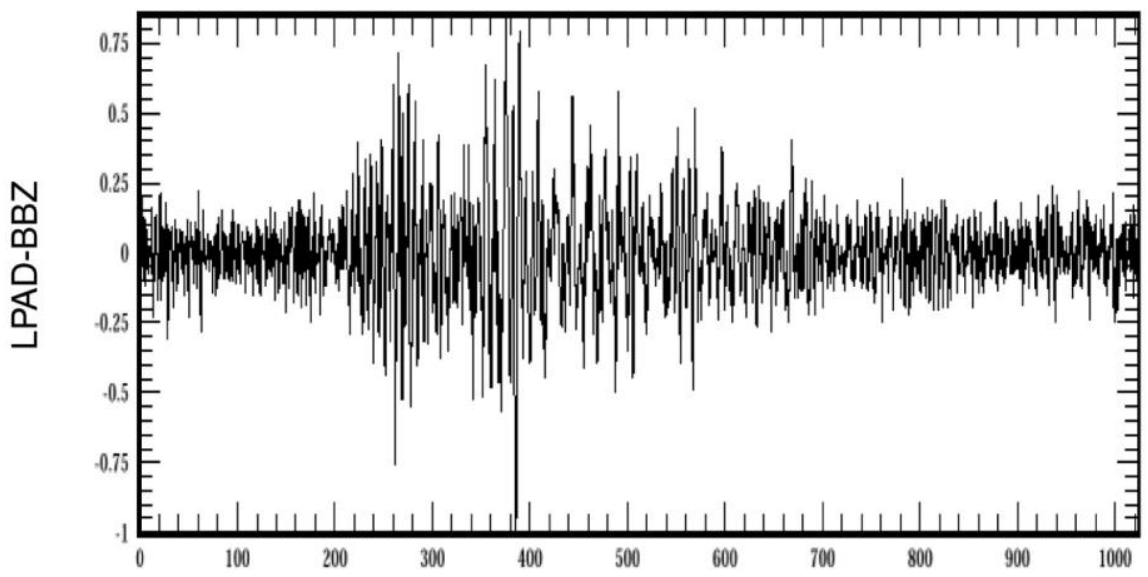

TIEMPO DESDE EL ORIGEN (seg)

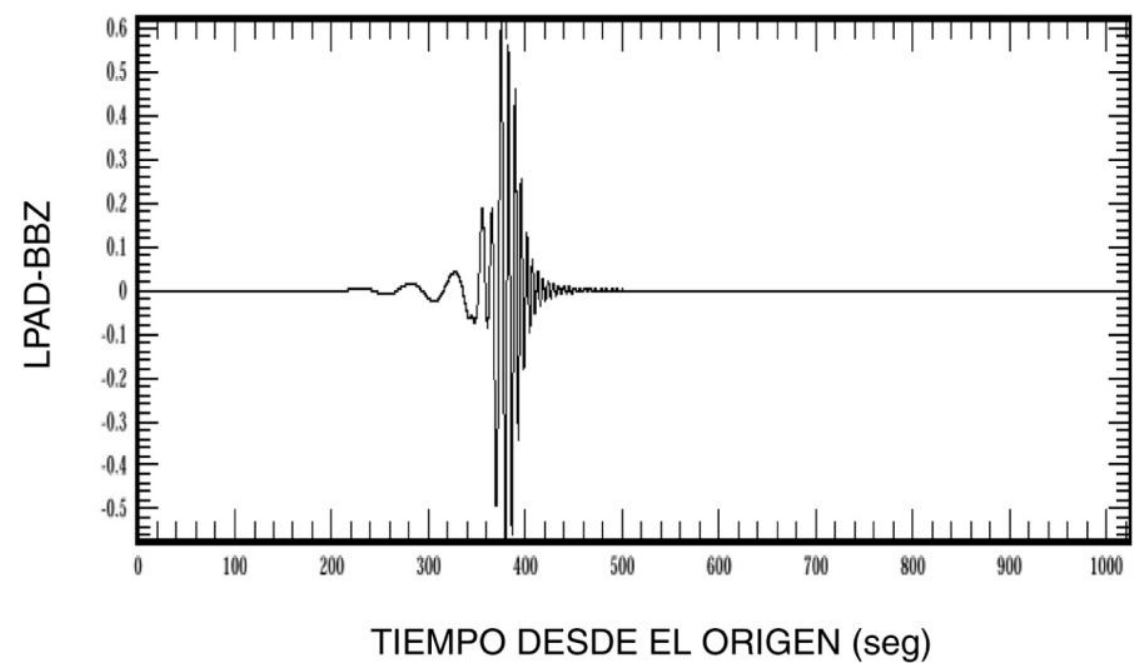

Figura 7.6. Entrada al FTAN y la señal filtrada para el evento del 3 de julio de 1996. 
Como ejemplo del proceso de la segunda etapa, se muestra en la Fig. 7.5 el dato original correspondiente a un evento del Set 1 y en la Fig. 7.6 la entrada al FTAN y la señal filtrada del mismo evento.

\subsection{Curvas de dispersión}

La Fig. 7.7 muestra las curvas de dispersión obtenidas para los distintos eventos correspondientes a los cinco Sets seleccionados. Las diferencias en las curvas asociadas con los otros Sets, indican claramente que las diferencias estructurales en la corteza y manto superior pueden ser resueltas mediante este método.
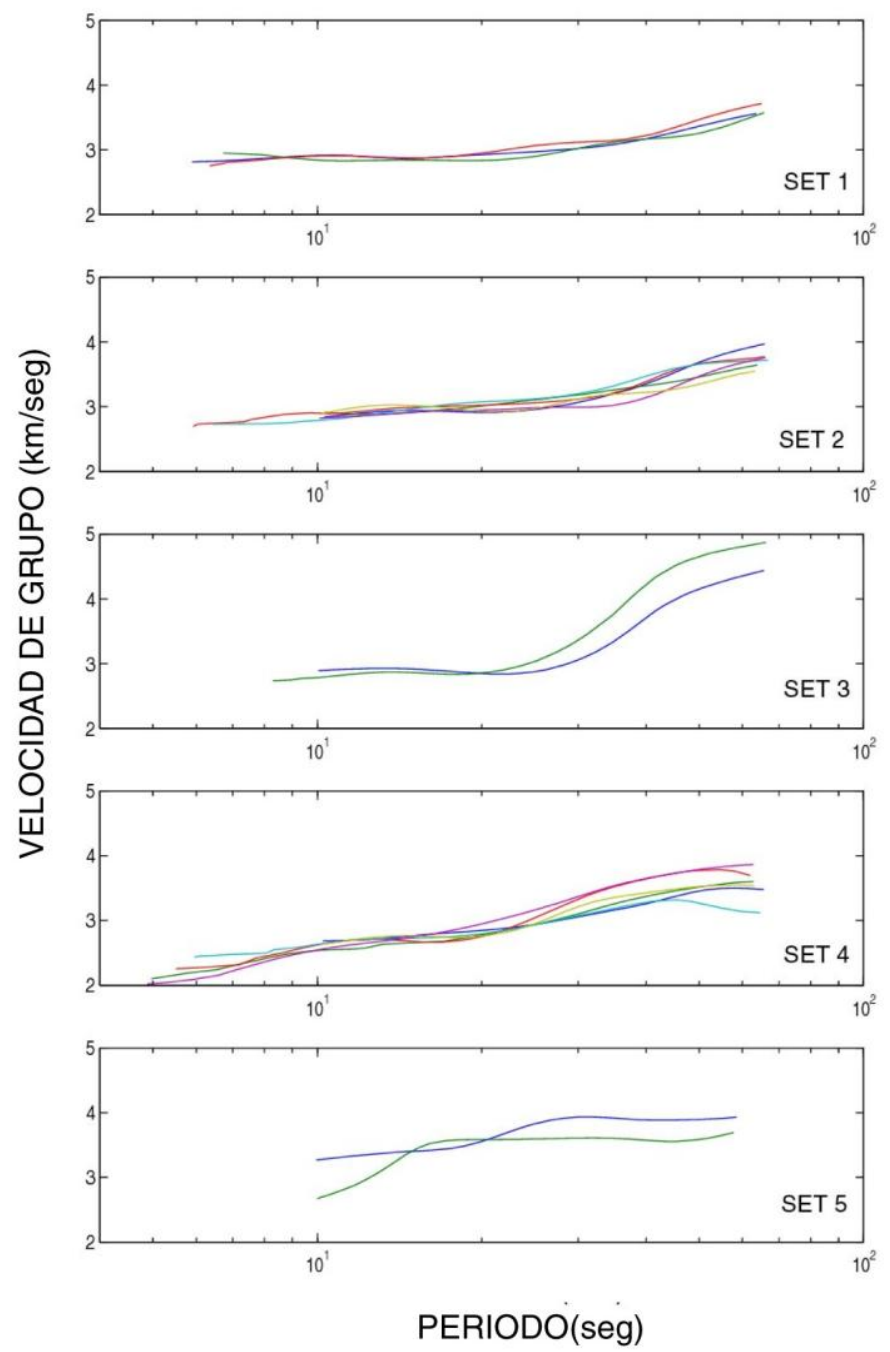

Figura 7.7. Curvas de dispersión para los distintos eventos correspondientes a los 5 Sets seleccionados. 


\subsection{Inversión}

A efectos de obtener los modelos finales, se usó el método de inversión desarrollado por D. Lokshtanov (1993), basado en la técnica descripta por Kushnir y otros (1988). Estos autores asumen que el medio es homogéneo horizontalmente y en consecuencia proponen un sistema lineal:

$$
U_{\text {out }}(w)=H(w, m) \cdot U_{\text {in }}(w)
$$

que relaciona las señales modeladas en la estación de entrada $U_{\text {in }}(w)$ y salida $U_{\text {out }}(w)$, a través de la matriz de respuesta en frecuencia del medio $H(w, m)$, donde $m$ corresponde a los parámetros que caracterizan al medio. Para poder resolver el problema inverso, se selecciona una familia parametrizada de funciones $H(w, m)$ para encontrar la respuesta en frecuencia que estadísticamente permita obtener los mejores estimadores de los parámetros desconocidos $\mathrm{m}$.

El programa requiere para su funcionamiento un modelo cortical de entrada, donde se especifica el número de capas, la velocidad de la onda $\mathrm{P}$ y de la onda $\mathrm{S}$, así como la densidad y el espesor de cada capa.

Como modelos de entrada para el proceso de inversión se utilizaron los correspondientes al CRUST 5.1, (Mooney y otros, 1998). En el mismo se presenta un mapa del espesor de la corteza en una escala de 5 grados por 5 grados que fue obtenido utilizando datos de refracción sísmica ubicados en todo el mundo. Sin embargo, aunque este es un estudio extenso, el mayor número de perfiles usados se ubica en el hemisferio norte. Eso implica que los resultados, especialmente para América del Sur, están basados en estudios globales de características tectónicas similares más que en mediciones locales. No obstante lo cual, los datos propuestos nos permiten tener una primera aproximación para los modelos de partida.

Luego se modificaron estos modelos de partida de manera de obtener modelos más confiables que ajusten mejor con las curva de dispersión. Para ello fue necesario cambiar el espesor de las capas, en algunos casos, agregar nuevas capas y cambiar la velocidad de las ondas; teniendo en cuenta que siempre la última capa representa el manto. Para los Sets 1, 2 y 3 los modelos que mejor ajustan tienen cinco capas, para los otros dos Sets, 4 y 5 , los modelos tienen seis capas.

Para cada Set, se invirtieron las distintas curvas de dispersión para todos los eventos y se eligieron los modelos que ajustaron mejor.

En la Fig. 7.8 se muestran las correspondientes curvas de dispersión teóricas y calculadas y en la Fig. 7.9 se muestran los modelos finales para cada Set. 
Analizando los resultados se encuentra que para el Set 1 el espesor cortical resulta aproximadamente igual a $52 \mathrm{~km}$, para el Set 2, $55 \mathrm{~km}$, para el Set 3, $44 \mathrm{~km}$, para el Set 4, $34 \mathrm{~km}$ y para el Set 5, $32 \mathrm{~km}$.
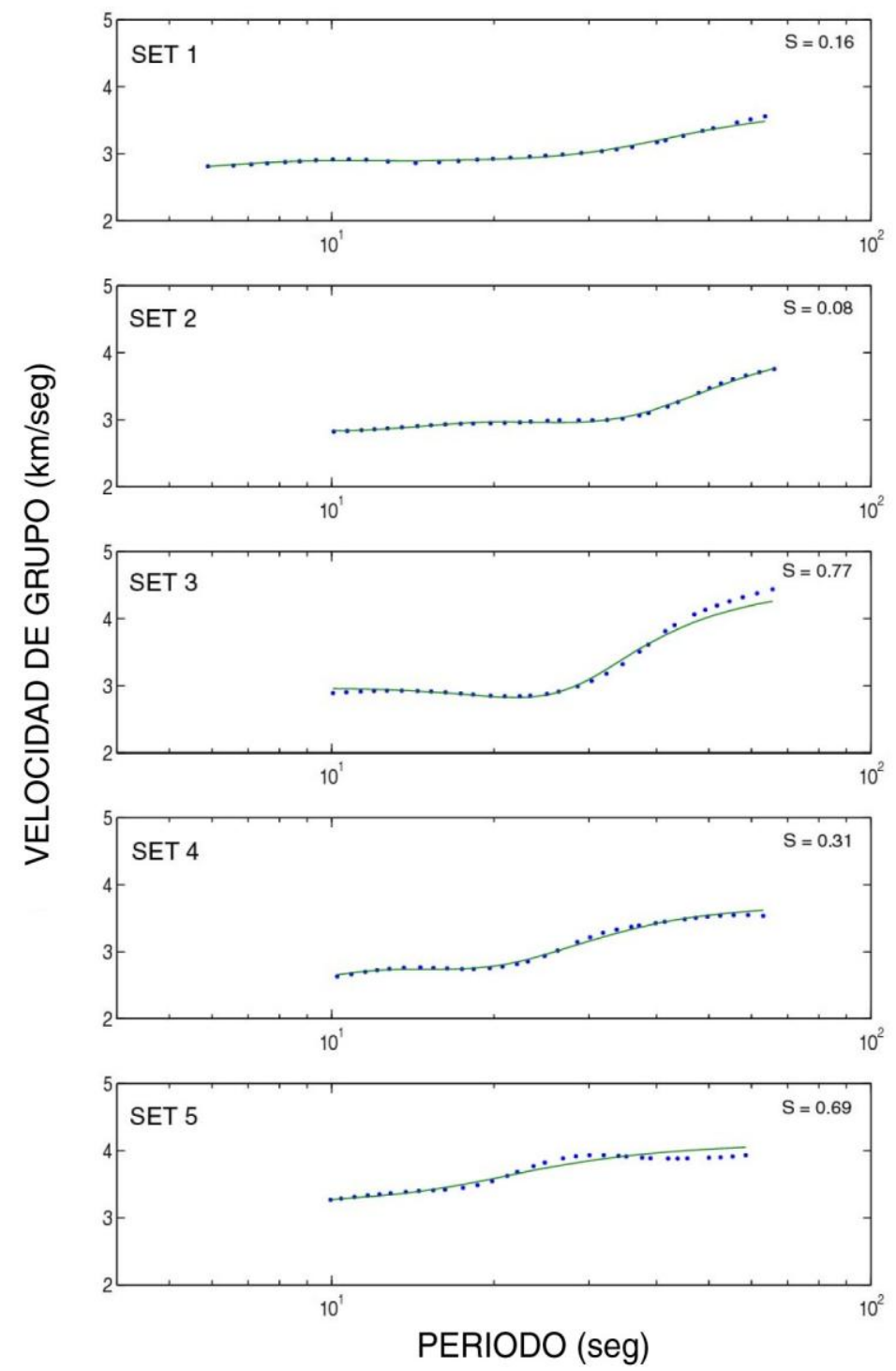

Figura 7.8. Resultados de la inversión correspondiente a los distintos Sets. La velocidad de grupo de ondas Rayleigh calculada en puntos y la teórica en línea llena. En cada Set se indica el parámetro de ajuste s calculado a partir de los residuos en el retardo.

La bondad del proceso de inversión se cuantifica a través del parámetro $\mathrm{s}$, calculado a partir de los residuos del retardo. Este parámetro, que se ha incluido en la Fig. 7.8 posee los valores $0.16,0.08,0.77,0.31$ y 0.69 para los Sets 1 a 5 , respectivamente. 
Se observa que en los Sets 1 y 2 que incluyen la región de las Sierras Pampeanas, el espesor cortical varía entre 52 y $55 \mathrm{~km}$ mostrando una gran similitud en los modelos de ambas zonas y en correspondencia con el espesor hallado para esta región por Regnier y otros (1994).

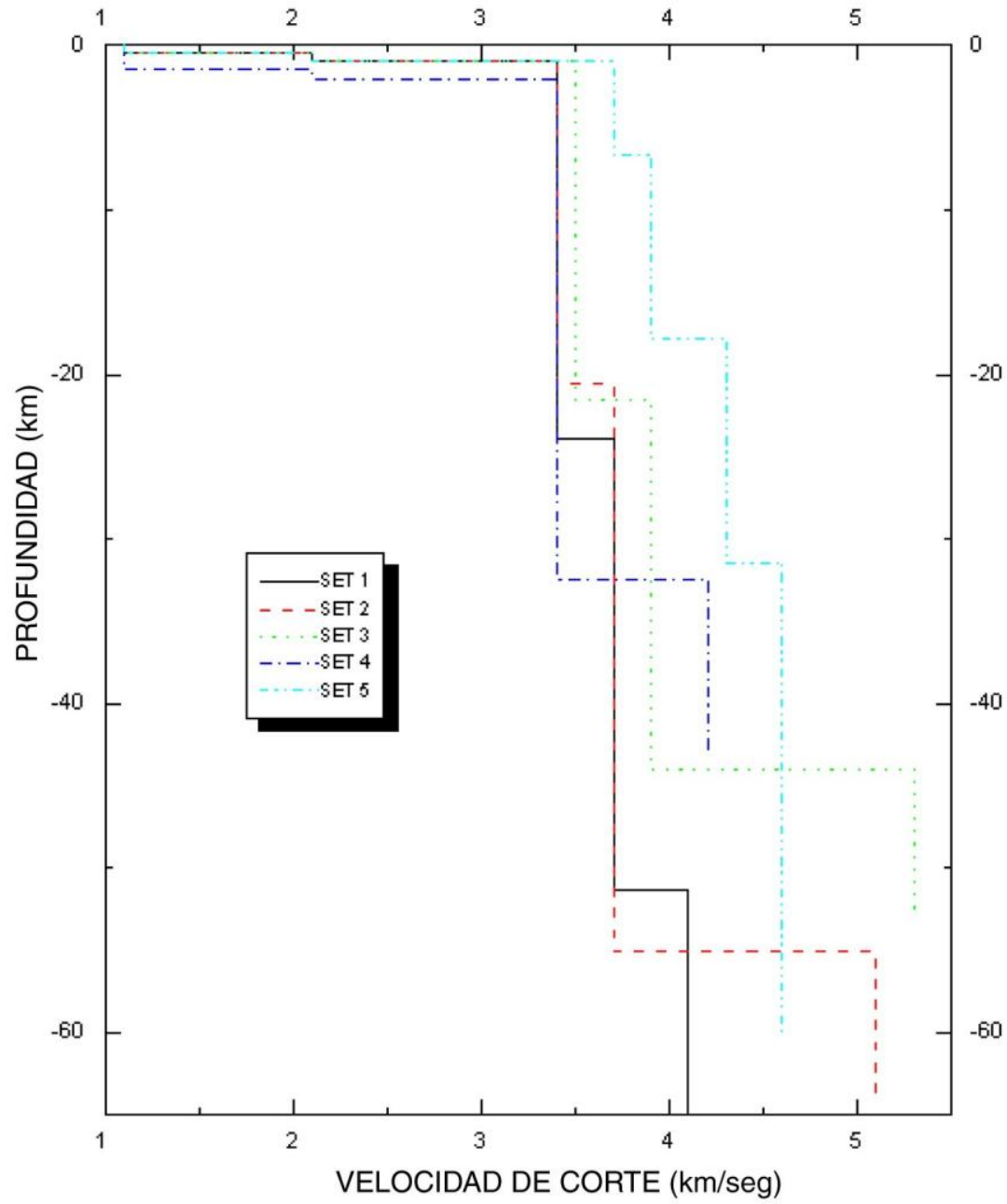

Figura 7.9. Mejores ajustes de modelos corticales obtenidos luego de la inversión de las curvas de dispersión, para cada Set.

A efectos de corroborar estos resultados y teniendo en cuenta que para el período considerado no había otras estaciones sismológicas al este de las Sierras Pampeanas, se analizó con el mismo procedimiento eventos registrados en CPUP, estación ubicada en Villa Florida, Paraguay $\left(26^{\circ} .33 \mathrm{~S}, 57^{\circ} .33 \mathrm{~W}\right)$, perteneciente a IRIS. En la Fig. 7.10a se muestran las curvas de dispersión obtenidas que luego fueron 
invertidas. Las estructuras resultantes obtenidas con el mejor ajuste fueron comparadas con las obtenidas para los mismos datos de LPA. Las Figs. 7.10b y 7.10.c ilustran el ajuste y la comparación de los modelos corticales, respectivamente. Se puede ver claramente la concordancia entre ambos modelos.

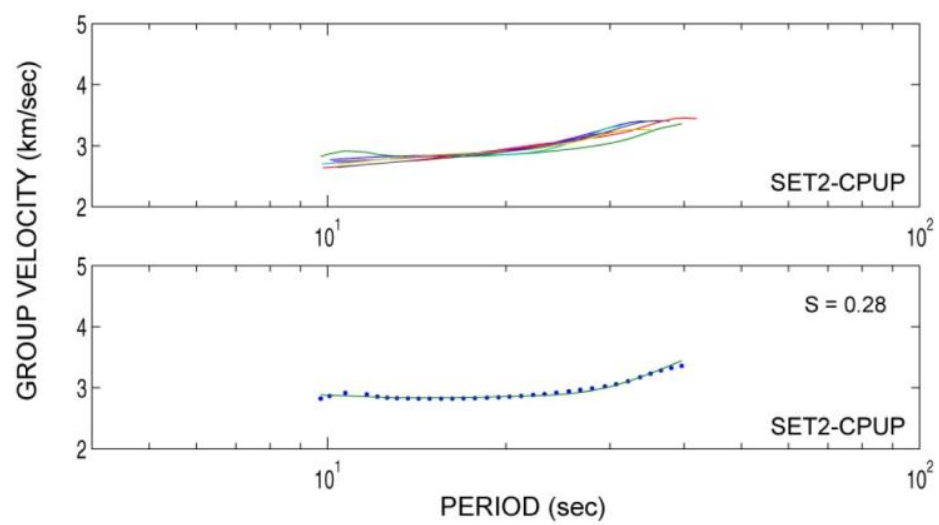

(a)

(b)

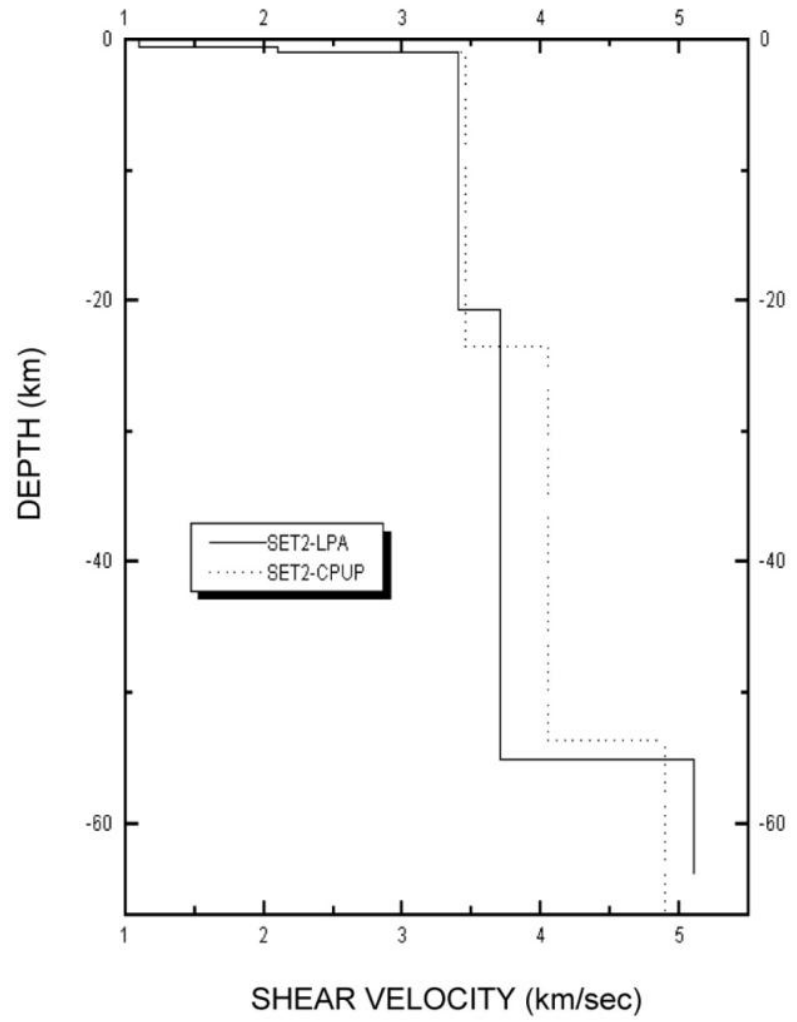

(c)

Figura 7.10. a) Curvas de dispersión para los distintos eventos correspondientes al Set 2, registrados en CPUP. b) Velocidad de grupo de ondas Rayleigh calculada (puntos) y teórica (línea llena). c) El mejor ajuste de modelo cortical correspondiente a trayectorias entre el Set 2 y ambas estaciones sismológicas, LPA y CPUP.

\subsection{Resultados y discusión}


Los resultados obtenidos para eventos correspondientes a los Sets 1 y 2 indican una profundidad de Moho que se aproxima a los valores obtenidos por otros métodos geofísicos. El mapa regional de anomalías de Bouguer que cubre un cinturón entre $69^{\circ}$ y $64^{\circ} \mathrm{W}$ a una latitud de $27^{\circ} \mathrm{S}$ en el margen occidental, y de $28^{\circ} 30^{\prime} \mathrm{S}$ en el límite oriental, muestran variaciones del espesor cortical desde los $40 \mathrm{~km}$ en el escudo Brasileño hasta los $70 \mathrm{~km}$ debajo de Los Andes, con un valor medio de aproximadamente 45-50 km debajo de las Sierras Pampeanas (Toselli y otros, 1999). Teniendo en cuenta que esta área coincide con trayectorias del Set 1, las profundidades estimadas concuerdan con los valores encontrados en este trabajo.

Los valores que se obtienen de la sismicidad, así como de ondas convertidas, (Smalley y otros, 1993; Regnier y otros, 1994), dan un valor aproximado de espesor cortical de $55 \mathrm{~km}$ para esta región. En particular, el estudio llevado a cabo por Regnier y otros (1992), se centró en la Sierra Pie de Palo, en el limite sur de las Sierras Pampeanas. Comparando este resultado con los valores hallados para el Set 2, se observa que hay una total coincidencia. Además, ambos resultados se diferencian de los predichos para esta región por el modelo CRUST 5.1 (Mooney y otros, 1998), de $70 \mathrm{~km}$. Sin embargo, esta diferencia era previsible ya que este modelo global posee fundamentalmente dos limitaciones. La primera de ellas es el tamaño de las celdas, 5ำ x 5을 que cubre un área suficientemente grande como para poder incluir dos estructuras corticales distintas dentro de una celda y, por otra parte, la ausencia de perfiles de refracción en la mayor parte del continente sudamericano, lo cual impide una resolución local de las estructuras.

Se ve también que las velocidades de manto para estos dos Sets presentan diferencias. Se ha encontrado que las velocidades de la onda transversal en el manto superior para las trayectorias del Set 1 (cuenca norte incluyendo la Planicie tucumana) tienen valores bajos, o sea de carácter tectónico o astenosférico, mientras que las velocidades para las trayectorias del Set 2 son más altas, características de los escudos. De acuerdo a estudios de ondas Lg (Rial y Ritzwoller, 1997), las bajas velocidades en el manto superior pueden ser indicadores de altas temperaturas; siendo las $\mathrm{Lg}$ ondas que se propagan tal como ondas superficiales que dominan los sismogramas de terremotos débiles regionales para trayectorias continentales.

La interpretación de este fenómeno podría vincularse con los datos corticales existentes. Aunque todas las trayectorias cruzan las Sierras Pampeanas, las cuencas intermontanas correspondientes a este sistema revelan características diferentes entre ellas, y estas diferencias pueden ser la explicación de los resultados obtenidos. 
Los datos gravimétricos y magnetotelúricos han provisto información de la estructura de las cuencas principales ubicadas en este sistema, incluyendo el espesor de las cubiertas sedimentarias y en algunos casos la profundidad del Moho. Los resultados mostraron diferentes características cuando se tuvieron en cuenta transectas que cruzaran las Sierras Pampeanas en diferentes latitudes.

Estudios magnetotelúricos realizados en las diferentes cuencas indicaron que éstas eran muy profundas y conductivas, con un espesor de la cubierta sedimentaria de aproximadamente 9 km (Pomposiello y otros, 1998, 2002). Estos rasgos son típicos de otras cuencas occidentales pertenecientes a este sistema, y valores de espesor similares han sido obtenidos a través de mediciones gravimétricas, refracción y reflexión sísmicas en 29.7오 y en 31.5오 (Jordan y Allmendinger, 1986; Introcaso, 1990).

Además, se ha detectado la presencia de una zona de muy alta conductividad, asociada a la existencia de una importante área termal en la región correspondiente a los eventos del Set 1 (Osella y otros, 1992, 1993; Pomposiello y otros, 1994, 2002; Toselli y otros, 1999).

Se pueden comparar los valores predichos por la inversión de las velocidades de grupo de ondas Rayleigh con los resultados anteriormente mencionados. El espesor de la cubierta sedimentaria que se ha encontrado es de aproximadamente 1 km. El bajo espesor sedimentario obtenido luego del proceso de inversión puede ser explicado por el hecho que el valor resultante es el promedio a lo largo del camino completo, el cual incluye no solo valles, sino también cordones montañosos.

La manifestación termal en superficie podría ser el resultado de un aumento de temperatura en el manto. Este hecho podría explicar las bajas velocidades encontradas en el Set 1 y la diferencia de velocidades con el Set 2.

Por otra parte, en el Set 3, se observa un resultado diferente a aquellos obtenidos para los Sets 1 y 2 (Figs. 7.7 y 7.8). El mayor ajuste para la inversión arroja un valor de $44 \mathrm{~km}$ para el espesor cortical con una velocidad de $\mathrm{S}$ para el manto superior de $5.3 \mathrm{~km} / \mathrm{seg}$, que es mayor que los valores obtenidos para los Sets 4 y 5 . El hecho que las trayectorias incluidas en el Set 3 son prácticamente cratónicas podrían explicar este resultado. Sin embargo, estas explicaciones son especulativas si consideramos que en este caso han sido analizados pocos sismogramas.

Por el contrario, cuando se tratan los eventos del Set 5 , se encuentra un comportamiento distinto. Se puede observar que el espesor cortical decrece hacia el sur en Los Andes; los valores de espesor obtenido son menores que en las Sierras 
Pampeanas. Además, la relación entre los resultados del Set 3 y del Set 5 puede ser explicada si tomamos en cuenta que los epicentros del Set 3 están ubicados cerca del margen oceánico, en la zona de subducción, mientras que los eventos del Set 5 se originaron lejos de la zona andina y se propagaron a través de recorridos cratónicos, lo que significa que el espesor cortical es menor, similar al resultado obtenido para el Set 4. Sin embargo, se ha notado que los resultados para el Set precedente tienen las mismas limitaciones que los del Set 3.

Los resultados obtenidos con epicentros en Bolivia (Set 4), cuyas trayectorias se encuentran en regiones cratónicas, se aproximan a resultados previos y corresponden a una corteza más delgada, como era previsible. Snoke y James (1997) encontraron que el espesor cortical en la cuenca de Chaco era sólo de aproximadamente $32 \mathrm{~km}$, espesor que es sustancialmente menor que el de la cuenca del Paraná, con velocidades llamativamente bajas. También se ha encontrado una velocidad baja, de aproximadamente $4.2 \mathrm{~km} / \mathrm{seg}$, en la parte superior del manto, similar a lo que Snoke y James (1997) encontraron debajo de la cuenca del Chaco, un valor que es característico de manto astenosférico. Estos autores atribuyeron el significado de la aparente baja velocidad en el manto superior a dos posibles causas: la primera conectada con el hecho que las bajas velocidades pueden ser atribuidas al arco andino, la segunda relacionada al hecho que en particular en la cuenca del Chaco, ubicada en la región del arco donde la expansión del retroarco ocurre debajo del arco de islas oceánicas, la estructura de velocidades, tanto cortical como de manto superior, pueden ser indicadores de manto más caliente y quizás acortamiento cortical. Por el contrario, el único tectonismo asociado con el retroarco de Los Andes, incluyendo los terremotos corticales subandinos, parecen ser de origen compresional y no extensional, y nosotros podríamos en consecuencia, no esperar extensión debajo de la cuenca chaquense.

\subsection{Conclusión}

Se ha usado el análisis de dispersión de ondas superficiales para investigar las propiedades físicas del sistema litósfera-astenósfera en la región de las Sierras Pampeanas y comparado los resultado con aquellos obtenidos para otras estructuras tectónicas. Aunque sólo se utilizó una estación de registro, este estudio es el primero para obtener estimaciones regionales del Moho basado en el análisis de velocidades 
de ondas superficiales y los modelos 1D obtenidos de la inversión de las curvas de dispersión están de acuerdo con resultados previos.

Las trayectorias de los Sets 1 y 2 pueden considerarse de espesor cortical similar pero con diferentes velocidades. Esto es el reflejo de la existencia de una área termal además de una anomalía eléctrica en la corteza profunda detectada por otros métodos geofísicos, para la trayectoria entre el Set 1 y La Plata.

Se ha encontrado también un espesor preliminar de $44 \mathrm{~km}$ para las trayectorias entre el Set 3 y La Plata.

En los Sets 4 y 5 , que incluyen eventos cuyos caminos atraviesan zonas cratónicas, se han encontrado espesores corticales menores, de aproximadamente 32 km. La profundidad del Moho obtenida a partir del Set 4 está de acuerdo con la obtenida previamente para la cuenca de Chaco.

Finalmente, vale la pena recalcar que este estudio aporta información obtenida con ondas superficiales a través del método de una única estación. Aún con las limitaciones propias de este método, la información que provee es útil, especialmente si se tiene en cuenta la poca información sísmica de la región, en comparación con América del Norte y Eurasia. 


\section{Capítulo 8}

\section{EL MOVIMIENTO CAUSADO POR LOS SISMOS DE MAYOR MAGNITUD DE LA ZONA CENTRAL DE CHILE EN EDIFICIOS ALTOS DE BUENOS AIRES}

\section{1 - Introducción}

Aunque desde 1920 han estado en operación estaciones sismológicas en la ciudad de Buenos Aires y de La Plata, separadas 50 kilómetros una de otra, debido a su baja sismicidad, ni en la ciudad de Buenos Aires ni en otras ciudades cercanas fueron instalados acelerógrafos de terremotos.

Los anteriores movimientos por efecto de sismos de gran magnitud y epicentro distante que fueron percibidos en los edificios altos de Buenos Aires saturaron la capacidad de registro del instrumental sismológico instalado en el área, por lo que no pudo obtenerse información acerca de las amplitudes, frecuencias y duración de los movimientos generados por estos importantes eventos.

Durante los años 1997-1998 se instalaron los acelerómetros de banda ancha BB13 con su adquisidor en la Estación Sismológica LPA, previo a su traslado a la Estación Sismológica TRWA (Sabbione, 2004). Estos sensores captaron, entre otros, eventos de mayor magnitud ocurridos en la zona central de Chile, que fueron percibidos en los pisos superiores de los edificios más altos de la ciudad de Buenos Aires generando, según información de diarios locales, sólo una visible oscilación de cuadros ubicados en paredes y de artefactos de luz colgantes del techo de habitaciones o, también, la apertura de puertas de algunos equipamientos de oficinas y muebles del hogar, aunque no han sido detectados daños estructurales.

En particular en este estudio, como una aplicación del uso de datos digitales, se analizan los registros en aceleración de terremotos que ocurrieron el 15-10-1997 con $\mathrm{Mw}=7,1$, el 29-07-1998 con $\mathrm{Mw}=6,4$ y el 03-09-1998 con $\mathrm{Mw}=6,6$, provenientes de la zona costera de Chile central (USGS-NEIC).

Los acelerogramas presentan un amplio contenido de frecuencias que es la consecuencia de tres factores: el mecanismo con que se desarrolla la ruptura, las particularidades de transmisibilidad que posee el medio por el que viajan las ondas sísmicas en su trayectoria desde el foco hasta el punto de captación y las propiedades dinámicas de los estratos de terreno sobre los que está ubicada la estación sismológica. En general, a medida que aumenta la distancia entre el foco y la estación, tiende a disminuir el contenido de las mayores frecuencias y se extiende la duración 
del movimiento por efecto de las reflexiones, refracciones y dispersiones que se producen en la trayectoria de las ondas sísmicas. Por otra parte, en la estación, los estratos superiores de suelos blandos amplían los contenidos de las menores frecuencias y como tal pueden afectar más selectivamente a las construcciones que posean particularidades dinámicas sensibles a tales frecuencias.

Teniendo en cuenta además que los movimientos sísmicos son excitaciones dinámicas que actúan sobre los edificios, sus respuestas serán una consecuencia tanto de sus propiedades dinámicas como de las particularidades de la excitación. Entre las propiedades dinámicas de estos edificios se consideran sus períodos fundamentales de vibración.

Un ejemplo destacado de la suma de los efectos del mecanismo de foco con la trayectoria de la ondas, con las propiedades de los terrenos blandos superficiales y las propiedades dinámicas de algunos edificios, lo constituye la ciudad de Méjico. Allí el sismo de gran magnitud que se produjo a $400 \mathrm{~km}$ de distancia epicentral ocasionó, en 1985, el colapso de edificios de gran altura debido al contenido y amplitud de las bajas frecuencias del movimiento resultante (USGS- Earthquake Hazards Program, Lomnitz, 1988, entre otros).

El objetivo del presente estudio es determinar los valores de aceleración a la que han sido sometidos ciertos edificios altos de la ciudad de Buenos Aires, al momento de estar excitados por las ondas sísmicas generadas en ocasión de los eventos mencionados previamente. En tal sentido, se hará primeramente una descripción de la sismicidad del litoral chileno (zona donde han ocurrido los eventos utilizados para el presente estudio) y de los efectos que ocasionaron algunos de estos sismos en la ciudad de Buenos Aires y alrededores; luego se describirán las características dinámicas de algunos edificios altos medidas previamente, para finalmente calcular los espectros de respuesta a partir de los acelerogramas obtenidos en la estación sismológica LPA.

\section{2 - Sismicidad}

\subsubsection{Litoral chileno.}

En la zona de subducción de la Placa de Nazca bajo la Placa Sudamericana, han ocurrido en el siglo pasado sismos que superaron la magnitud Ms=8, tales como el de 1906 que dañó a Valparaiso y el de 1943 que afectó a Illapel. El sismo más reciente de gran magnitud ocurrido en esta zona es el del 3 de marzo de 1985 que con 
$\mathrm{Mw}=7,9$ produjo daños y pérdidas de vida en las ciudades de Valparaiso y Santiago y en sus áreas adyacentes (USGS-NEIC).

A lo largo de la costa de Chile existen grandes diferencias en la sismicidad, en la morfología de la costa y en el potencial de generación de grandes tsunamis (Fig. 8.1). Se puede caracterizar la sismicidad de Chile en tres zonas en función de las latitudes:

1) zona Norte (18ㅇ $\left.-27^{\circ} \mathrm{S}\right)$

2) zona Central (27으 $\left.-37^{\circ} \mathrm{S}\right)$

3) zona Sur (37오 - 60은

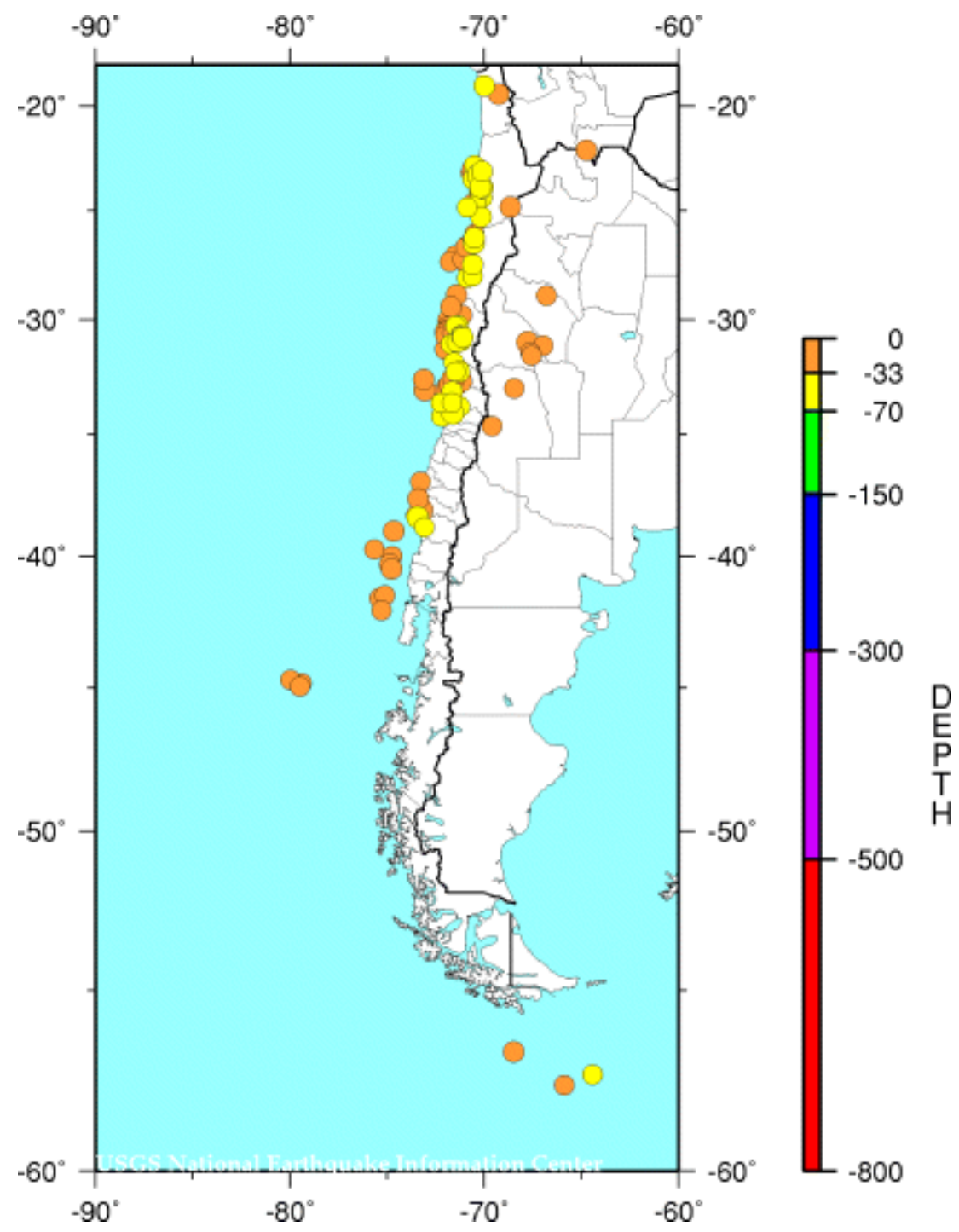

Figura 8.1: Mapa de sismicidad superficial para el período 1973 al presente de eventos con magnitud mayor o igual a 6.0. Fuente USGS-NEIC (PDE) 
1) La zona Norte de Chile puede ser dividida en tres regiones sísmicas bien diferenciadas. La zona más al norte comprende las áreas entre las latitudes $18^{\circ} \mathrm{S}$ y $20^{\circ} \mathrm{S}$, la segunda región sísmica incluye el área entre los $20^{\circ} \mathrm{S}$ y $22^{\circ} \mathrm{S}$, y la tercera región sísmica incluye el área entre los $22^{\circ}$ y $27^{\circ} \mathrm{S}$.

a) La región de más al Norte $\left(18^{\circ}\right.$ a $\left.20^{\circ} \mathrm{S}\right)$ tiene una activa historia de sismos destructores y de maremotos o tsunamis. Grandes terremotos en 1604, 1705, 1868 y 1877 destruyeron Arica, así como otros pueblos costeros, y generaron tsunamis que fueron destructivos en casi todo el Océano Pacífico.

b) En la segunda región sísmica del Norte de Chile (20 a $\left.22^{\circ} \mathrm{S}\right)$ han ocurrido sismos costeros y hacia el interior. Los sismos costeros han generado varios tsunamis, pero ninguno de ellos ha sido destructivo.

c) La provincia de Antofagasta $\left(22^{\circ}-27^{\circ} \mathrm{S}\right)$ incluye mucha actividad sísmica de profundidad intermedia $(70-300 \mathrm{~km}$ ), pero carece de grandes sismos. Los eventos más grandes en esta área ocurrieron el 4 de diciembre de 1918 y el 28 de diciembre de 1966.

2) La zona Central, conocida al norte como la zona de los valles transversales, está caracterizada por la ausencia de volcanismo del Cuaternario y por la ocurrencia de tsunamis destructivos, cada 20 años, durante el presente siglo.

En esta región los eventos más importantes ocurrieron el 8 de julio de 1730, el 11 de abril de 1819, el 11 de noviembre de 1922, el 6 de abril de 1943, el 19 de abril de 1955 y el 9 de julio de 1971. Los eventos de 1730 y 1922 generaron tsunamis que causaron daños en lugares tan alejados como Japón.

En la región cerca de Valparaíso $\left(30^{\circ}-34^{\circ} \mathrm{S}\right)$, que está marcada por la intersección de la Cordillera submarina de Juan Fernández con la fosa y por la aparición brusca del volcanismo del Cuaternario hacia el Sur, se produjeron cinco terremotos productores de tsunamis: el 19 de noviembre de 1811, el 19 de noviembre de 1822, el 16 de octubre de 1868, el 17 de agosto de 1906 y el 3 de marzo de 1985 (Tabla 8.1). Los terremotos de 1822 y de 1906 se centraron casi en la misma ubicación; ambos tuvieron magnitudes de 8,5 o más en la Escala de Richter y ambos causaron daños.

En la región al Sur de Valparaíso (34ํㅡㄴ $\left.36^{\circ} \mathrm{S}\right)$, existe una brecha sísmica de tamaño moderado (ausencia de sismos grandes en los últimos años) y el potencial de probabilidad de ocurrencia de sismos futuros es alto. Cuatro eventos de magnitud igual o superior a 7,5 en la Escala de Richter han ocurrido en esta área en 1871, 1923, 1928 y 1975 , pero ninguno de ellos produjo tsunami. 


\begin{tabular}{|c|c|c|c|c|c|c|}
\hline Año & Mes & Día & Long. ${ }^{-W}$ & Lat. ${ }^{\circ} \mathrm{S}$ & Magnitud & Efectos \\
\hline 1687 & & 12 & 32,8 & 70,7 & 7,3 & \\
\hline 1730 & 07 & 08 & 32,5 & 71,5 & 8,7 & Tsunami importante \\
\hline 1751 & 05 & 25 & 36,5 & 74,0 & 8,0 & Tsunami importante \\
\hline 1811 & 11 & 19 & 33,0 & 71,4 & & Tsunami pequeño \\
\hline 1819 & 04 & 11 & 27,0 & 71,5 & 8,5 & Tsunami importante \\
\hline 1822 & 11 & 19 & 33,0 & 71,6 & 8,5 & Tsunami importante \\
\hline 1835 & 02 & 20 & 36,8 & 73,0 & 8,2 & Tsunami importante \\
\hline 1838 & 05 & 07 & 36,7 & 73,3 & & Tsunami pequeño \\
\hline 1849 & 12 & 17 & 29,9 & 71,4 & 7,5 & Tsunami importante \\
\hline 1851 & 05 & 26 & 27,0 & 71,6 & 7,2 & Tsunami mediano \\
\hline 1859 & 10 & 05 & 27,0 & 70,0 & 7,7 & Tsunami importante \\
\hline 1868 & 09 & 14 & 36,7 & 73,2 & & Tsunami pequeño \\
\hline 1868 & 10 & 16 & 33,1 & 71,1 & & Tsunami pequeño \\
\hline 1871 & 03 & 25 & 35,0 & 72,5 & 7,5 & Tsunami pequeño \\
\hline 1878 & 02 & 14 & 36,8 & 73,0 & & Tsunami pequeño \\
\hline 1906 & 08 & 17 & 33,0 & 72,0 & 8,6 & Tsunami importante \\
\hline 1918 & 05 & 20 & 28,5 & 71,5 & 7,9 & \\
\hline 1922 & 11 & 07 & 28,0 & 72,0 & 7,0 & \\
\hline 1922 & 11 & 11 & 28,5 & 70,0 & 8,3 & Tsunami importante \\
\hline 1923 & 02 & 17 & 35,3 & 72,4 & & Tsunami pequeño \\
\hline 1923 & 05 & 04 & 28,7 & 71,7 & 7,0 & \\
\hline 1928 & 12 & 01 & 35,0 & 72,0 & 8,4 & Tsunami mediano \\
\hline 1931 & 03 & 18 & 32,5 & 72,0 & 7,1 & \\
\hline 1939 & 01 & 25 & 36,3 & 72,3 & 8,3 & \\
\hline 1939 & 04 & 18 & 27,0 & 70,5 & 7,4 & \\
\hline 1943 & 04 & 06 & 30,8 & 72,0 & 8,3 & Tsunami pequeño \\
\hline 1953 & 05 & 06 & 36,5 & 72,5 & 7,6 & \\
\hline 1955 & 04 & 19 & 30,0 & 72,0 & 7,1 & Tsunami pequeño \\
\hline 1971 & 07 & 07 & 32,5 & 71,2 & 7,5 & \\
\hline 1975 & 05 & 10 & 35,7 & 74,6 & 7,8 & \\
\hline 1983 & 10 & 04 & 26,5 & 70,6 & 7,4 & Tsunami pequeño \\
\hline 1985 & 03 & 03 & 33,2 & 72,0 & 7,8 & Tsunami pequeño \\
\hline 1985 & 03 & 03 & 32,7 & 71,2 & 7,0 & \\
\hline 1985 & 04 & 09 & 34,1 & 71,6 & 7,5 & \\
\hline 1997 & 10 & 15 & 30,9 & 71,2 & 7,6 & \\
\hline
\end{tabular}

Tabla 8.1: Historia sísmica de la zona Central de Chile para eventos de $\mathrm{M}>6,9$. (modificada de Lorca Mella y Recabarren Herrera, 2003)

La región de Concepción (36ํㅜ - 37오 S) muestra dos eventos en 1835 y 1939 cuyas magnitudes han sido superiores a 8 en la Escala de Richter, el primero de los 
cuales generó un tsunami destructor. A ellos se agregan eventos de magnitud menor en $1751,1868,1869,1878,1953$ y 1961.

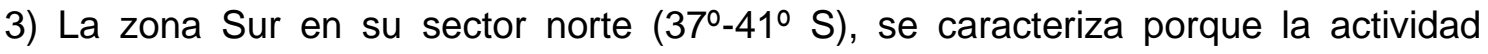
sísmica principal se ha desplazado hacia mar afuera. Grandes eventos se han registrado en ella el 28 de octubre de 1562, el 8 de febrero de 1570, el 16 de diciembre de 1575, el 15 de marzo de 1657 y el 22 de mayo de 1960. De ellos, los de 1562, 1570, 1575 y 1960 produjeron tsunamis destructores. Los intervalos entre estos terremotos parecen ser mayores que los producidos en las áreas de Concepción y Valparaíso. El terremoto del 22 de mayo de 1960, de magnitud 8,9 es el evento sísmico más grande en esta región desde 1570.

La región Sur puede subdividirse a su vez en tres subregiones:

a) La región desde $41^{\circ} \mathrm{S}$ hasta $45^{\circ} \mathrm{S}$ : la costa de Sudamérica cambia dramáticamente en la latitud $41^{\circ} \mathrm{S}$. Desde este punto hacia el Sur, la línea de costa se hace irregular y tiene numerosas islas, bahías y caletas. En el extremo norte de esta región (cerca de la latitud $41^{\circ} \mathrm{S}$ ) ocurrieron terremotos que generaron tsunamis destructores en $1633 \mathrm{y}$ 1837. En 1929, ocurrió otro tsunami destructor en la latitud $44.6^{\circ} \mathrm{S}$.

b) La región desde los $45^{\circ} \mathrm{S}$ hasta los $53^{\circ} \mathrm{S}$ : al sur de la unión triple entre la Fosa Chile y la Dorsal de Chile en la latitud $46^{\circ} \mathrm{S}$, donde la parte oceánica de la Placa Antártica está siendo transportada bajo la Placa Sudamericana a una razón de 2 $\mathrm{cm} / a n ̃ o$. Existen evidencias paleomagnéticas en el Pacífico Sur que indican que segmentos de la Dorsal de Chile colisionaron con la parte más austral del Continente Sudamericano hace 26 millones de años. Desde esa época ha habido una disminución de la actividad volcánica en el continente y un cese del plegamiento en las cuencas sedimentarias.

\subsection{2 - Ciudad de Buenos Aires y alrededores}

La ciudad de Buenos Aires es al área metropolitana más extensa y populosa de Argentina, con una población de cerca de 10 millones de habitantes; está situada en el este de la gran llanura formada a partir de potentes capas de sedimentos aluviales que constituyen la pampa argentina.

Está localizada sobre la margen derecha del Río de La Plata, aproximadamente a cien kilómetros de la desembocadura al Océano Atlántico.

De acuerdo a la información provista por catálogos internacionales (Fig. 8.2, Tabla 8.2), los eventos sísmicos cercanos de mayor magnitud registrados instrumentalmente ocurrieron a una distancia de 610 kilómetros a la ciudad de Buenos 
Aires, en Sampacho, provincia de Córdoba, en junio de 1934 y el 22 de mayo de 1936 en San Francisco del Monte, provincia de San Luis.

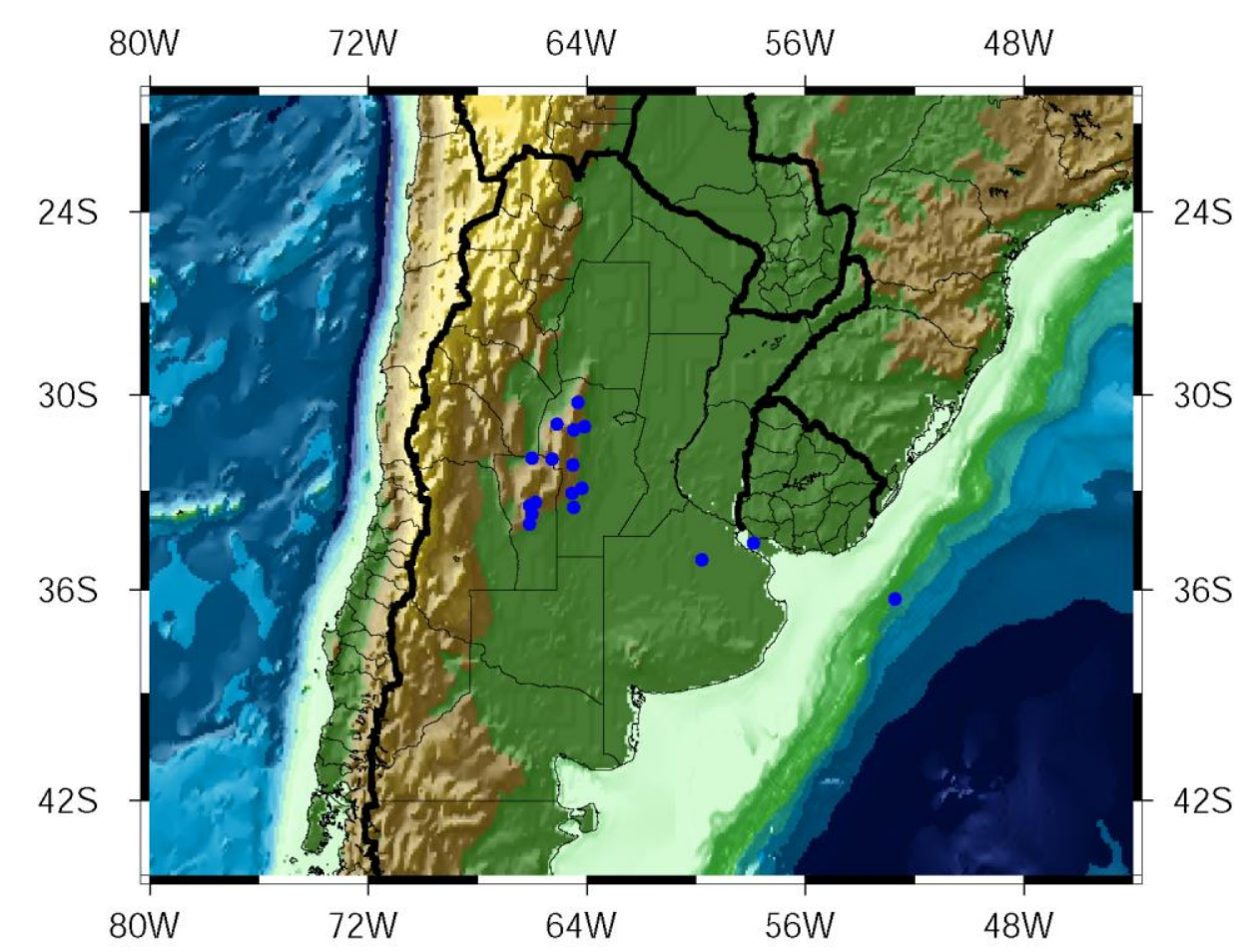

Figura 8.2: Mapa de sismicidad superficial hasta 800 km de Buenos Aires (USGS, modificado por Jaschek y otros, 1982)

Además, se han registrado instrumentalmente otros eventos sísmicos menores, a corta distancia epicentral de Buenos Aires; tal es el caso del evento del 2 de noviembre de 1971, sentido en Vieytes, A. Jonte y Magdalena, con una distancia a La Plata de $57 \mathrm{~km}$ al SE (Jaschek, 1972).

En esta Tabla debe hacerse notar que el evento del 28 de octubre de 1965 no figura en los catálogos del USGS y del ISC, mientras que el evento del 15 de febrero de 1967 sólo figura en el ISC con una determinación preliminar dada por LASA, lo que hace que estos dos eventos tengan un peso relativo menor.

Esta información sismológica acerca del área cercana a la ciudad de Buenos Aires muestra que no han ocurrido hasta la fecha sismos de magnitudes mayores a 6.0. Después de más de cuatrocientos años desde su fundación, no han llegado a nuestros días referencias acerca de algún terremoto que haya ocasionado víctimas fatales o daños graves en la ciudad o en sus alrededores. Sólo en pocas ocasiones se han sentido algunos temblores. El más destacado de ellos fue el que ocurrió hace algo más de una centuria, en los primeros minutos (HOA) del 5 de Junio de 1888 y que 
sacudió a la ciudad de Buenos Aires. Según noticias periodísticas (diario La Prensa) produjo dos sacudimientos con una diferencia de pocos segundos que hizo que los muebles crujieran, los cuadros colocados en los muros se balancearan y aún las paredes se sacudieran, moviéndose visiblemente, además parecía que los ángulos de las esquinas de las habitaciones se desfiguraban, siendo en las casas altas donde el temblor se sintió con mayor fuerza.

\begin{tabular}{|c|c|c|c|c|c|c|c|}
\hline CAT. & $\begin{array}{l}\text { FECHA } \\
\text { aa } \mathrm{mm} \text { dd }\end{array}$ & $\begin{array}{l}\text { HORA } \\
\text { hh mm ss }\end{array}$ & LAT. & LONG. & $\begin{array}{c}\text { PROF. } \\
(\mathrm{km})\end{array}$ & MAG. & $\begin{array}{r}\text { DIST } \\
(\mathrm{km})\end{array}$ \\
\hline SISRA & 18451019 & 19 & -35.100 & -59.800 & 30 & $5.00 \mathrm{MsPSA}$ & 177 \\
\hline SISRA & 18880605 & $03: 20$ & -34.600 & -57.900 & 30 & $5.50 \mathrm{MsPSA}$ & 14 \\
\hline SISRA & 19340611 & 03:07:09 & -33.500 & -64.500 & 30 & 6.00MsPAS & 610 \\
\hline SISRA & 19340611 & 06:00:33 & -33.500 & -64.500 & 30 & 6.00MsPAS & 610 \\
\hline SISRA & 19360522 & 00:15:58 & -32.000 & -66.000 & 40 & 6.00MsPAS & 794 \\
\hline SISRA & 19470116 & 02:37:40 & -31.10 & -64.50 & 50 & 5.50MsPSA & 715 \\
\hline SISRA* & 19651028 & $22: 57: 25$ & -36.62 & -64.28 & 40 & $4.60 \mathrm{bPSA}$ & 443 \\
\hline SISRA* & 19670215 & $16: 44: 33$ & -35.600 & -58.000 & 33 & $5.00 \mathrm{mb} / \mathrm{SC}$ & 121 \\
\hline SISRA & 19670917 & 06:37:08.70 & -34.030 & -66.100 & 12 & $4.10 \mathrm{mblSc}$ & 747 \\
\hline SISRA & 19741006 & $14: 40: 56.10$ & -30.93 & -65.09 & 40 & $4.69 \mathrm{mblSC}$ & 772 \\
\hline SISRA & 19750128 & 06:32:26 & -33.082 & -64.553 & 50 & $4.1 \mathrm{mbGS}$ & 626 \\
\hline PDE & 19831024 & 15:09:54.92 & -32.01 & -65.29 & 33 & $4.70 \mathrm{mb}$ GS & 732 \\
\hline PDE & 19860205 & $01: 12: 22.04$ & -30.22 & -64.34 & 10 & $4.80 \mathrm{mb}$ GS & 761 \\
\hline PDE & 19870325 & 02:43:20.80 & -32.91 & -64.18 & 29 & $4.50 \mathrm{mb}$ GS & 599 \\
\hline PDE & 19880626 & 03:24:25.82 & -36.27 & -52.73 & 31 & $5.10 \mathrm{mb}$ GS & 517 \\
\hline PDE & 19960729 & $00: 04: 47.28$ & -33.47 & -66.10 & 33 & $4.90 \mathrm{mb}$ GS & 756 \\
\hline PDE & 19980530 & 11:31:52.79 & -33.70 & -66.02 & 42 & $4.70 \mathrm{mb}$ GS & 744 \\
\hline PDE & 19991124 & $15: 37: 42.38$ & -33.35 & -65.88 & 33 & $4.50 \mathrm{mb}$ GS & 739 \\
\hline PDE & 20020315 & $21: 35: 48.37$ & -30.99 & -64.09 & 33 & $4.70 \mathrm{mb}$ GS & 690 \\
\hline PDE & 20030305 & \begin{tabular}{|l|}
$15: 22: 12.21$ \\
\end{tabular} & -32.20 & -64.51 & 33 & $4.10 \mathrm{mb}$ GS & 656 \\
\hline
\end{tabular}

Tabla 8.2: Sismicidad superficial con magnitud mayor o igual a 3.0 hasta una distancia de $800 \mathrm{~km}$ de la ciudad de Buenos Aires. Fuente: USGS, modificada (Jaschek y otros, 1982). ( ${ }^{*}$ peso relativo menor).

Fue percibido por las personas que se encontraban en reposo o en casas altas y no por las que circulaban por la calle. Fue sentido en las ciudades de Campana, La Plata y Buenos Aires, con una intensidad sísmica de IV en la escala Mercalli. Por lo anteriormente mencionado, de acuerdo a la información acerca de la percepción del movimiento, una mejor estimación del epicentro es que se localizó sobre el Delta del Río Paraná, mientras que su magnitud se puede considerar de 4.4 (Gershanik, 1996). 
También algunas veces se han percibido a nivel de terreno suaves movimientos oscilatorios de poca amplitud y relativamente larga duración, que han correspondido a eventos sísmicos distantes y con grandes magnitudes. Uno de ellos, quizás el más destacado, es el sismo del 27 de Octubre de 1894, cuya área epicentral se localizó en el noroeste del territorio de la Provincia de San Juan, cerca del limite con Chile, a 1100 kilómetros de Buenos Aires. De acuerdo con la extensión del área en que se produjeron daños y la gran distancia hasta la cual fue nítidamente percibido, su magnitud fue estimada como 7.7 (Gershanik, 1996) y por ello, éste es el evento sísmico de mayor magnitud que haya ocurrido en el territorio argentino. En esa oportunidad, el diario La Nación editado en Buenos Aires, informó acerca de su percepción en esta ciudad “... Las damas fueron las más impresionadas por el fenómeno. Muchas de ellas, quienes estaban caminando por las calles, de repente sintieron náuseas y comenzaron a buscar refugio en las entradas de las casas y de los negocios...".

En varias ocasiones, el movimiento oscilatorio del terreno generado por eventos sísmicos distantes y con grandes magnitudes sólo fue claramente percibido en los niveles superiores de los edificios más altos en la ciudad de Buenos Aires. Así, el Diario La Nación informó de esta percepción durante el terremoto del 15 de Enero de 1944 que destruyó a la ciudad de San Juan y causó mas de 8000 muertes, cuya magnitud alcanzó a Ms=7,2 y su área epicentral se localizó a 1000 kilómetros de Buenos Aires. Quizás, el más desagradable de estos movimientos ocurrió durante el sismo del 23 de Noviembre de 1977 que afectó a Caucete en la provincia de San Juan, cuya magnitud alcanzó a Ms=7,4 y cuyo epicentro se localizó a 950 kilómetros desde Buenos Aires, (Carmona y otros, 1978) evento en el cual varios de los habitantes de los niveles superiores de los edificios de 20 y 30 pisos los abandonaron muy alarmados por la amplitud y duración del movimiento. En ocasión de este sismo colapsó y se destruyó la torre del tanque de agua de la Acería de Bragado (Pcia de Bs.As).

Los eventos sísmicos mencionados anteriormente tuvieron su área epicentral en la parte oeste del territorio Argentino. Similarmente, los eventos sísmicos de gran magnitud con foco en el territorio Chileno también fueron nítidamente percibidos en los niveles superiores de los edificios de la ciudad de Buenos Aires, tal como durante el gran sismo del sur de Chile del 22 de mayo de 1960, el de mayor magnitud registrado en el mundo en el siglo $X X, M w=9,6$, y también durante el último de ellos ocurrido el 3 de Marzo de 1985 en la zona central de Chile con magnitud Mw=7.9. 
Para tener una idea acerca de la sensibilidad de los ocupantes de los edificios altos en la ciudad de Buenos Aires con relación a los eventos sísmicos distantes, es apropiado mencionar que el sismo del 9 de Junio de 1994, que se localizó en territorio boliviano a 650 kilómetros de profundidad y con magnitud $M w=8,2$, cuyo epicentro distó 3000 kilómetros desde Buenos Aires, fue también levemente percibido en algunos de sus edificios más altos.

Para citar un ejemplo de las percepciones sentidas en caso de temblores, se reproduce (en el apartado) parte de una nota del diario El Clarín del 19 de junio de 2002.

\begin{abstract}
"Un temblor que sacudió la zona central de Chile poco antes de las 11 de ayer hizo sentir sus coletazos en pleno centro porteño, donde algunos edificios fueron evacuados por prevención. El sismo tuvo epicentro en la costa chilena, a unos $400 \mathrm{~km}$ de Santiago, y los registros lo ubicaron en los 6,5 grados de la escala Richter y 6 en la escala Mercalli. En Buenos Aires, el movimiento produjo temor entre quienes se encontraban en los pisos más altos de las torres, sobre todo por no entender qué ocurría,.. En el centro porteño los primeros edificios evacuados fueron los de Bouchard al 500. También bajaron apresuradamente las escaleras los empleados de los edificios de Catalinas, donde tienen sus oficinas el Bank Boston e IBM, entre otras firmas. Lo mismo ocurrió en la torre de Chacabuco y Avenida de Mayo. En todos ellos, los relatos fueron similares.,, Diego (22) estaba en la oficina de su jefe, en el piso 12 del edificio HSBC, de Avenida de Mayo y Chacabuco, cuando se empezó a marear. Pensó que tenía un bajón de presión, pero enseguida se dio cuenta de que en realidad estaba temblando el edificio...pensó que esa mole vidriada de 24 pisos, se desplomaría a sus espaldas. Otros creyeron que era un simulacro de incendio. En el piso 22, Mariana (24) sintió algo más que un mareo. "Fue algo espantoso, estaba sentada frente a mi escritorio y la silla empezó a moverse de un lado a otro.... En pocos minutos estuvo en la calle, como tantos otros desorientados. Los vidrios crujían, las oficinas temblaban, y el mareo se hizo insoportable, daba náuseas... En la torre Bouchard, de Bouchard y Lavalle, frente al Luna Park, donde están las oficinas de Aerolíneas Argentinas entre otras empresas, se vivieron escenas similares. "Las cortinas se movían y los vidrios temblaban"... El temblor se sintió del piso 14 en adelante. "Alguien de seguridad pidió que desalojáramos el edificio... En los pisos más altos la mayoría de los empleados dijo que se sentían mareados. "Era como si estuviéramos en un barco: veíamos que el piso y las paredes se movían y estábamos muy mareados", contó un empleado del piso 23. Casi 400 empleados de los edificios Catalinas, en Retiro, también fueron evacuados. "Era como una oscilación, parecido a navegar. Por un momento creía que se movía hasta el piso"...
\end{abstract}

\title{
8.3 - Seis edificios altos de la ciudad de Buenos Aires
}

\subsubsection{Características}

Como en cada gran concentración urbana, la ciudad de Buenos Aires tiene edificios de diferentes alturas, algunos de los cuales, al presente, superan los 100 metros. Las estructuras de estos altos edificios son de hormigón armado y sus fundaciones están adaptadas al tipo de suelo pampeano sobre el que yacen.

Los vientos que soplan en el área son relativamente moderados, aunque en algunas ocasiones se convierten en tornados; por otra parte, la actividad sísmica que se desarrolla en el área circundante es de bajo nivel. Debido a estas circunstancias, las estructuras de estos edificios son diseñadas para fuerzas horizontales que son bastante menores que las empleadas en los edificios de otras áreas con conocida 
actividad sísmica, resultando estructuras bastante menos rígidas que las de edificios similares ubicados en importantes metrópolis sudamericanas de la región andina.

En un estudio previo de particularidades dinámicas de los rascacielos de la ciudad de Buenos Aires, se seleccionaron seis (Carmona y otros, 1995), todos ellos con una planta tipo que se repite en casi toda la altura por lo que su descripción geométrica es simple:

1.- Edificio Torre Le Parc: Es un edificio torre con una altura de $158 \mathrm{~m}$ y está dentro de los más altos de la ciudad; de hecho fue el edificio mas alto de Argentina entre los años 1996 (año de finalización de su construcción) y 2002. El edificio se desarrolla en dos niveles de sótanos, un entrepiso técnico, 43 plantas típicas, 4 plantas en dúplex, una losa superior de terraza con un tanque de reserva de agua de 165 metros cúbicos, un nivel para la maquinaria de los ascensores y una losa de coronamiento para helipuerto, totalizando así 53 niveles desde su fundación. Las losas de piso y la estructura han sido construídas con hormigón armado. La estructura está constituida por tabiques interiores y pórticos en su perímetro.

1. Edificio Torre Libertador 4440: Posee estructura de hormigón armado de 140 metros de alto. Su estructura portante está formada por dos núcleos de hormigón armado vinculados entre sí.

2. Edificio Chacofi: Es un edificio de 115 metros de alto cuya fundación está realizada con pilotes.

3. Edificio Torre Catalinas Norte: Este edificio de planta general cuadrada con 27 pisos y 108 metros de alto es una torre de líneas espigadas y simples. La estructura es de hormigón armado y está sustentada sobre un núcleo central y columnas perimetrales, con su fundación a 12 metros bajo del nivel de terreno.

4. Edificio Torre Bouchard: Destinado a oficinas con una planta cuadrada de 30 metros de lado con 30 pisos y un alto de 100 metros. Tiene una estructura de hormigón armado con un núcleo central de 12 metros de lado y un conjunto de 16 columnas perimetrales. Su fundación consiste en pilotes de gran diámetro.

5. Edificio Torre Pirelli: De los edificios aquí considerados, éste es uno con características distintivas. Es una torre de una planta pentagonal con casi 100 metros de alto constituido por 20 pisos destinados a oficinas, 2 pisos para servicios generales, 1 nivel panorámico y un helipuerto en la terraza. Su estructura es de hormigón armado y está formada por un núcleo de planta triangular con refuerzos en sus vértices sobre el cual apoyan una cenefa a nivel de basamento, dos pisos estructurales ubicados uno a media altura y el otro en el extremo superior. Cada 
piso estructural está formado por un haz de 8 ménsulas de hormigón postensadas empotradas al núcleo central. De cada uno de estos pisos estructurales están colgados los 8 pisos inferiores a ellos.

\subsubsection{Períodos fundamentales de vibración horizontal}

Para obtener los períodos fundamentales de la vibración horizontal de los edificios seleccionados, se captaron, registraron y analizaron las microvibraciones horizontales que ocurren en los niveles más altos a consecuencia de las excitaciones ambientales que permanentemente actúan sobre ellos, tales como el tránsito vehicular en sus inmediaciones, el movimiento de los ascensores o los vientos suaves a moderados que inciden sobre sus cerramientos exteriores (Carmona y otros, 1995).

Éste es un método simple, ampliamente empleado, fácil de aplicar y de bajo costo, aunque debe tenerse en cuenta que los resultados corresponden a niveles de excitación muy bajos y no podrían ser extrapolados para mayores niveles de excitación como son los efectos de los terremotos destructivos en zonas sísmicas, ya que la respuesta no es lineal.

En el caso que nos ocupa, el método es útil, siendo sus principales fundamentos la característica aleatoria de la excitación ambiental y el comportamiento del edificio como un vibrador lineal con baja amortiguación, de lo que resulta que el movimiento en los niveles superiores está predominantemente formado por los períodos fundamentales de vibración del edificio.

Con este objetivo, en alguno de los pisos superiores de cada uno de estos edificios seleccionados se instalaron sismógrafos portátiles de componente horizontal, con un período propio de $2 \mathrm{seg}$, que generaron archivos a 200 muestras por segundo. Las tomas de microvibraciones fueron extendidas durante unos poco minutos y repetidas en diferentes instantes. De esta información captada in-situ se seleccionaron los intervalos con mejor definición de los períodos mayores, a los que se les determinó su Transformada de Fourier, correspondiendo los valores más altos a los períodos de vibración buscados.

Con esta metodología relativamente simple se obtuvieron los períodos fundamentales de vibración horizontal de los seis edificios. En la Tabla 8.3 están detallados los valores de los períodos fundamentales de vibración horizontal obtenidos, mientras que en la Fig. 8.3 están representados los valores de estos períodos en función de la altura de cada edificio. 


\begin{tabular}{|l|c|c|c|}
\hline \multirow{2}{*}{ EDIFICIO } & ALTURA & \multicolumn{2}{c|}{ PERIODOS (seg.) } \\
\cline { 3 - 4 } & $(\mathrm{m})$ & Dirección 1 & Dirección 2 \\
\hline 1 - LE PARC & 160 & 2.7 & 2.3 \\
\hline 2 - LIBERTADOR 4440 & 140 & 2.6 & 2.5 \\
\hline 3 - CHACOFI & 115 & 2 & 2 \\
\hline 4 - CATALINAS NORTE & 108 & 2.4 & 2.3 \\
\hline 5 - BOUCHARD & 100 & 1.8 & 1.8 \\
\hline 6 - PIRELLI & 100 & 2.4 & 2.2 \\
\hline
\end{tabular}

Tabla 8.3 - Edificios Altos de la ciudad de Buenos Aires Periodos Fundamentales de Vibración Horizontal Obtenidos de Microvibraciones Ambientales (de Carmona y otros, 1995)

La relación resultante entre período y la correspondiente altura del edificio está limitada por los valores $0.014 \mathrm{~s} / \mathrm{m}$ y $0.024 \mathrm{~s} / \mathrm{m}$. Estos valores son mayores que aquellos obtenidos en edificios de similar altura con estructura de hormigón armado construidos en áreas sísmicas. [Carmona y Herrera, 1969], (Carmona y otros, 1995).

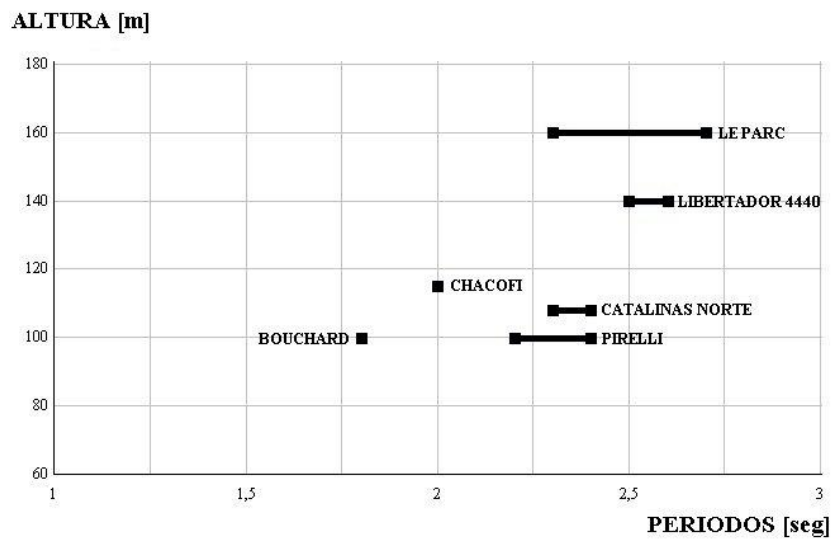

Figura 8.3 - Edificios altos de la ciudad de Buenos Aires. Periodos fundamentales de vibración vs altura (de Carmona y otros, 1995).

\section{4 - Efectos de sismos de mayor magnitud de la zona Central de Chile}

Los registros sísmicos analizados en este capítulo son tres de los de mayor magnitud que se han localizado en el litoral central chileno entre los años 1997-98.

Estos son los que ocurrieron el 15-10-1997 (UTC) con Mw=7,1, el 29-07-1998 con $\mathrm{Mw}=6,4$ y el 03-09-1998 con $\mathrm{Mw}=6,6$ (Tabla 8.4).

\begin{tabular}{|c|c|c|c|c|c|c|c|}
\hline FECHA & $\begin{array}{c}\text { HORA DE ORIGEN } \\
\text { (UTC) }\end{array}$ & LATITUD & LONGITUD & $\begin{array}{c}\text { Prof } \\
(\mathbf{k m})\end{array}$ & $\begin{array}{c}\text { Dist.(km) } \\
\text { La Plata }\end{array}$ & Mw & Ms \\
\hline $15-10-1997$ & $01: 33: 33.4$ & $30^{\circ}, 93 \mathrm{~S}$ & $71^{\circ}, 22 \mathrm{~W}$ & 58 & 1320 & 7,1 & 6,8 \\
\hline $29-07-1998$ & $07: 14: 24.1$ & $32^{\circ}, 31 \mathrm{~S}$ & $71^{\circ}, 29 \mathrm{~W}$ & 51 & 1270 & 6,4 & 6,3 \\
\hline $03-09-1998$ & $17: 37: 58.2$ & $29^{\circ}, 45 \mathrm{~S}$ & $71^{\circ}, 72 \mathrm{~W}$ & 27 & 1430 & 6,5 & 6,5 \\
\hline
\end{tabular}

Tabla 8.4 - Parámetros focales de los eventos que han sido seleccionados para el presente estudio. 
El 14 de Octubre de 1997, pocos minutos después de las 22hs locales, se percibió en los niveles superiores de edificios de Buenos Aires el movimiento no placentero previamente descripto, aunque con una intensidad menor que en ocasiones previas.

La causa fue el sismo de magnitud Mw=7.1 (USGS-NEIC) que había sacudido a la parte central de Chile. Las poblaciones más afectadas fueron Pueblo Nuevo y Punitaqui en donde además se produjeron siete muertos. En las ciudades de La Serena y Coquimbo cercanas al área epicentral también ocurrieron algunos daños.

Los otros dos sismos considerados tienen magnitudes $\mathrm{Mw}=6,4$ y 6,6 y aunque causaron alarma y daños menores en las poblaciones de sus áreas epicentrales, no ocasionaron víctimas fatales.

La Fig. 8.4 muestra los 360 segundos más importantes de las tres componentes del registro de aceleración obtenido en la Estación Sismológica LPA con los sensores BB13, para el sismo del 15 de octubre de 1997. La parte más intensa del movimiento duró aproximadamente 100 segundos en la componente $\mathrm{N}-\mathrm{S}$, que es la de mayor amplitud, y los valores de aceleración máxima resultantes en cada componente son, medidos en gal $=\mathrm{cm} / \mathrm{s}^{2}$ :

$$
\operatorname{Amax}(\mathrm{N}-\mathrm{S})=0,75 \mathrm{gal} ; \quad \operatorname{Amax}(\mathrm{E}-\mathrm{O})=0,53 \mathrm{gal} ; \quad \operatorname{Amax}(\mathrm{V})=0,27 \mathrm{gal}
$$

Estas aceleraciones máximas son sólo casi $1 / 1000$ de las aceleraciones máximas que se registran en las áreas epicentrales de los sismos cuyas magnitudes son similares a este evento sísmico. Por otro lado, estos bajos valores de aceleración justifican que el movimiento no fuera percibido a nivel de terreno en la ciudad de Buenos Aires.

Para los sismos del 29-07-1998 y del 03-09-1998, los valores de aceleración máxima resultante en cada componente registrados en La Plata son, en gal, respectivamente :

$$
\begin{array}{ll}
\operatorname{Amax}(\mathrm{N}-\mathrm{S})=0,14 \mathrm{gal} ; & \operatorname{Amax}(\mathrm{E}-\mathrm{O})=0,12 \mathrm{gal} ; A \max (\mathrm{V})=0,02 \mathrm{gal}, \\
\operatorname{Amax}(\mathrm{N}-\mathrm{S})=0,37 \mathrm{gal} ; & \operatorname{Amax}(\mathrm{E}-\mathrm{O})=0,19 \mathrm{gal} ; A \max (\mathrm{V})=0,09 \text { gal. }
\end{array}
$$

Estas aceleraciones máximas son menores que las correspondientes al sismo del 15-10-1997 y corresponden a movimientos que son imperceptibles para las personas que estén ubicadas a nivel del terreno. 

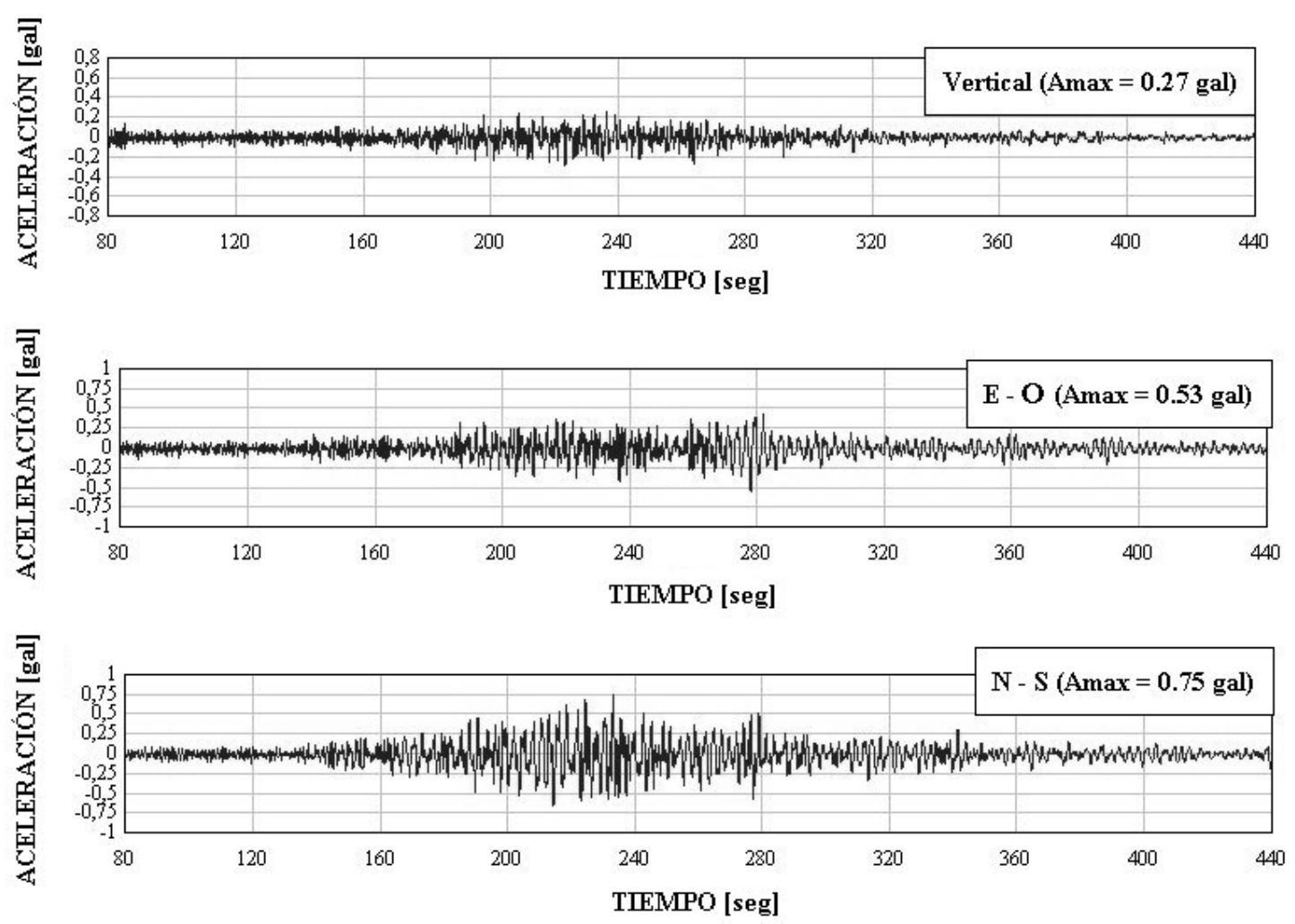

Figura 8.4 - Aceleración a Nivel de Terreno Registrada en la ciudad de La Plata Durante el sismo de Chile del 15 de octubre de 1997 (de Carmona y otros, 2000).

En la Fig. 8.5 están representadas las componentes N-S de estos tres sismos.

Se destaca la diferencia de la aceleración máxima y de la forma de la envolvente de estos dos últimos, no obstante tener magnitudes similares, ya que difieren en solo 0,2.

Otro aspecto a considerar de estos registros de aceleración es la duración de su parte significativa, considerada como el tiempo comprendido entre las aceleraciones mitad de la máxima previa y posterior a la misma. Los valores que resultan para esta duración significativa Ds por componente de cada sismo, son:

Sismo del 15-10-1997 : $\quad$ Ds $(N-S)=91 \mathrm{seg} ; \quad \operatorname{Ds}(E-O)=113 \mathrm{seg} ; \quad \operatorname{Ds}(Z)=293 \mathrm{seg}$

Sismo del 29-07-1998 : $\quad \mathrm{Ds}(\mathrm{N}-\mathrm{S})=134 \mathrm{seg} ; \quad \mathrm{Ds}(\mathrm{E}-\mathrm{O})=116 \mathrm{seg} ; \quad \mathrm{Ds}(\mathrm{Z})=302 \mathrm{seg}$

Sismo del 03-09-1998 : $\quad \mathrm{Ds}(\mathrm{N}-\mathrm{S})=31 \mathrm{seg} ; \quad \mathrm{Ds}(\mathrm{E}-\mathrm{O})=54 \mathrm{seg} ; \mathrm{Ds}(\mathrm{Z})=36 \mathrm{seg}$

Se observa que son comparables las duraciones significativas de las respectivas componentes de los sismos del 15-10-97 y del 29-07-98 no obstante que sus magnitudes difieren, ya que es $\mathrm{Mw}=7,1$ para el primero y $\mathrm{Mw}=6,4$ para el segundo, mientras que difieren notablemente las duraciones significativas de los sismos del 2907-98 y del 03-09-98 aunque sus magnitudes son similares. 
Capítulo 8: El movimiento causado por los sismos de mayor magnitud de la zona central de Chile en edificios altos de Buenos Aires.
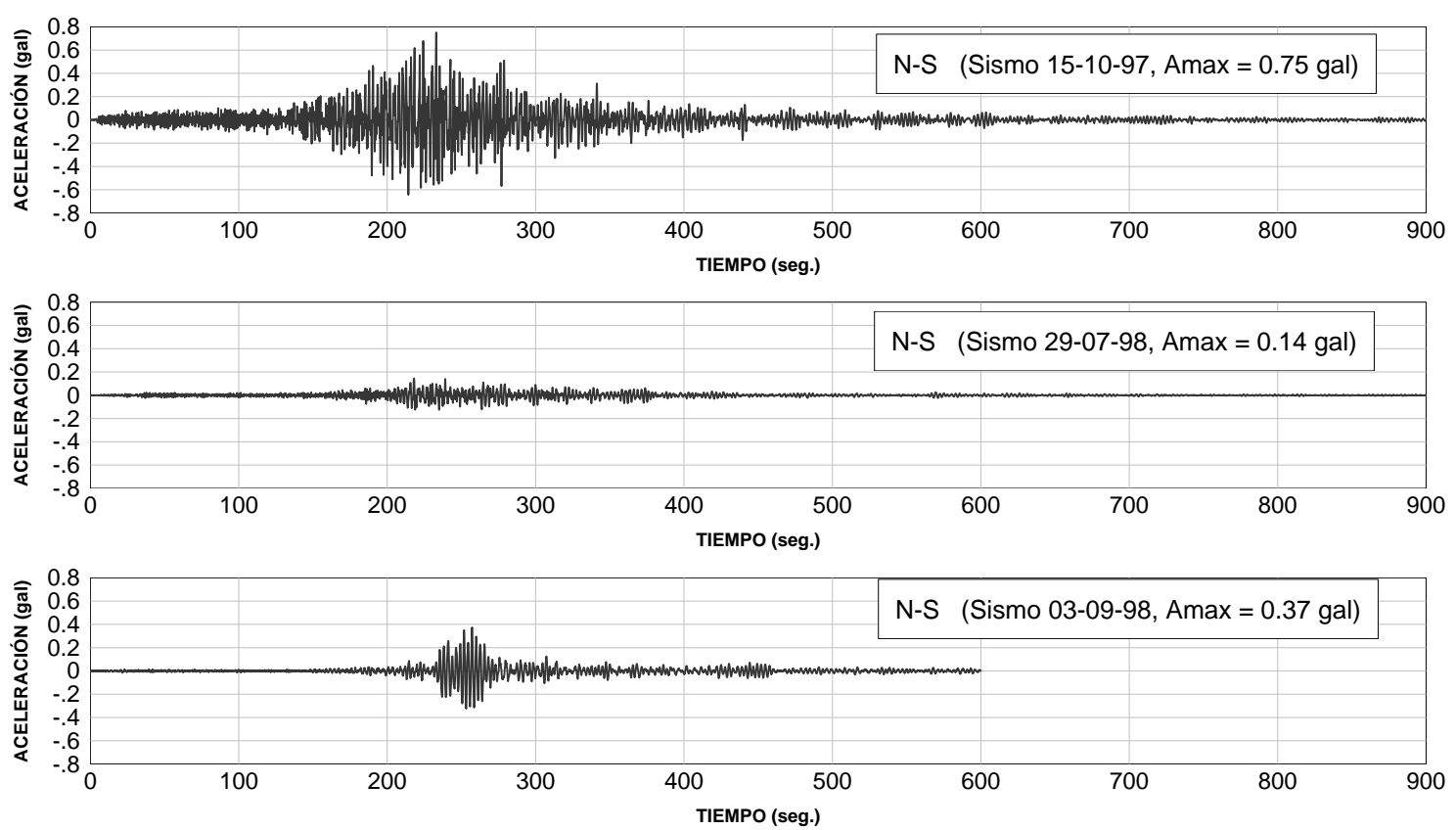

Figura 8.5 - Componentes N-S de la aceleración registrada en la ciudad de La Plata durante los sismos de Chile del $15-10-1997$, del 29-07-1998 y del 3-09-1998 (de Sabbione y otros, 2000).

En la Fig. 8.6 se presentan los espectros de las tres componentes de los correspondientes sismos suavizados mediante su promedio móvil. En los mismos se destaca la amplitud espectral para la frecuencia $\mathrm{f}=0,35 \mathrm{~Hz}$ que exhiben las componentes horizontales, mostrando así que estas ondas de período $T=1 / 0,35=2,86$ seg están fuertemente polarizadas en el plano horizontal.
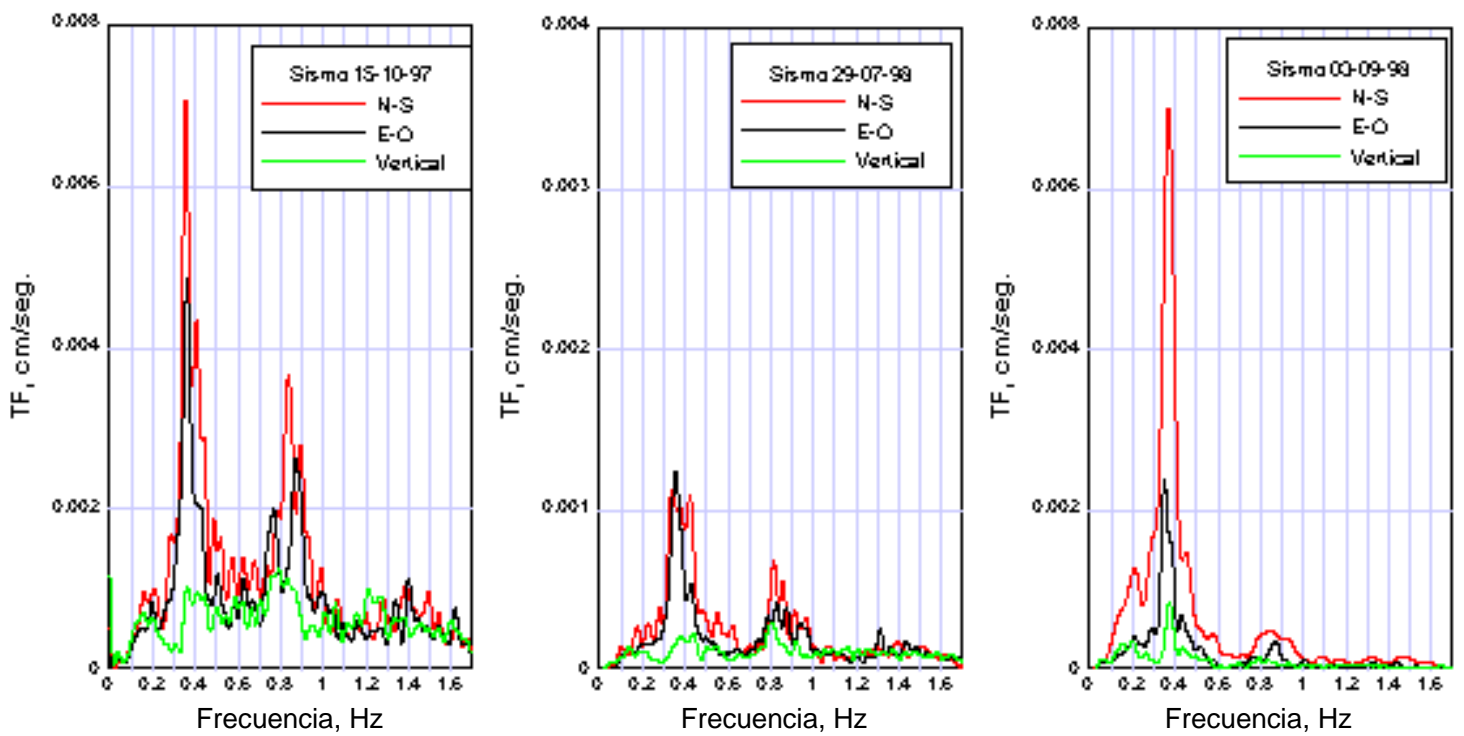

Figura 8.6 -Espectros de las aceleraciones registradas en la ciudad de La Plata durante los sismos de Chile del 15 -10-1997, del 29-07-1998 y del 3-09-1998 (de Sabbione y otros, 2000). 
Ya que componentes de esta frecuencia $\mathrm{f}=0,35 \mathrm{~Hz}$ están presentes en los distintos trenes de ondas cualquiera sea la velocidad del grupo, se puede considerar que las ondas de $\mathrm{f}=0,35 \mathrm{~Hz}$ son la consecuencia de las propiedades dinámicas de los estratos sedimentarios cercanos a la Estación Sismológica de La Plata. Por otra parte, las mayores aceleraciones coinciden con ondas de velocidad de grupo comprendidas entre 2,3 y 3,6 km/seg, que corresponden a las ondas $\mathrm{Lg}$ (Gershanik, 1996), y que resultan de las múltiples reflexiones de ondas $S$ confinadas en la corteza continental de gran espesor. Le sigue en importancia la amplitud para $\mathrm{f}=0,82$ a $0,85 \mathrm{~Hz}$, que es más intensa en el acelerograma del sismo del 15-10-97 y en el del 29-07-98 y en esos mismos acelerogramas una tercer frecuencia menos importante de $\mathrm{f}=1,29$ a 1,39 Hz.

Teniendo en cuenta que el sismo de Chile del 15 de octubre de 1997 ha provocado las mayores aceleraciones, se analizaron las curvas del espectro de respuesta de la aceleración absoluta para una amortiguación del $2 \%$ y períodos comprendidos entre 0,7 y 5 segundos (Fig. 8.7).

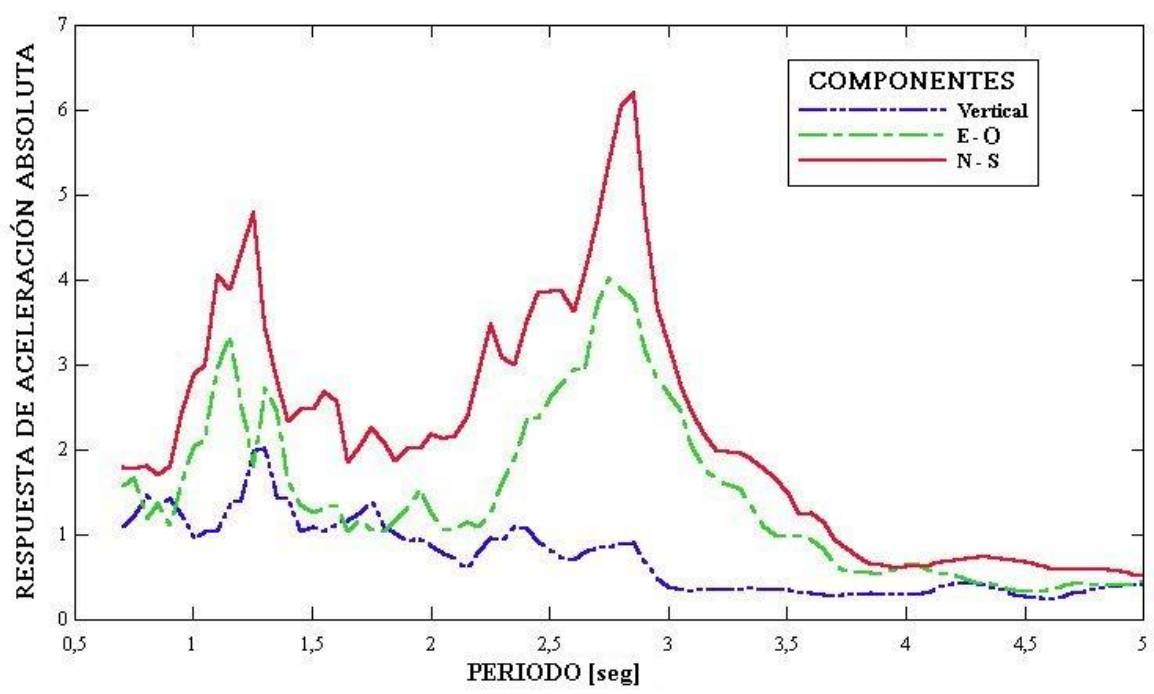

Figura 8.7 - Curvas de Respuesta de Aceleración Absoluta para 2\% de Amortiguación del Registro de Aceleración de La Plata del sismo del 15 de octubre de 1997 ( de Carmona y otros, 2000).

Las curvas correspondientes a las componentes horizontales del movimiento muestran que para los períodos cercanos a 1,2 y 2,7 seg ocurren los valores máximos de la aceleración de la respuesta. Estos valores alcanzan a ser de 6 a 8 veces mayores que el respectivo valor máximo de la aceleración registrada en cada componente y al menos 3 veces para períodos comprendidos entre ellos. Esta amplificación de 6 a 8 veces pone en evidencia la influencia de la duración de la parte más intensa del movimiento cuya aceleración está dada en la Fig. 8.4. 
Con el objeto de evaluar las amplitudes del movimiento en los niveles superiores de los edificios durante este terremoto, se deben realizar algunas consideraciones a partir de la información disponible.

a) El registro de aceleración obtenido en la ciudad de La Plata, Fig. 8.4, debería ser similar en contenido y distribución de frecuencias y amplitudes al acelerograma que se habría obtenido durante este terremoto en cada uno de los niveles de fundación de los edificios aquí estudiados. Esta es una suposición razonable porque ambas ciudades, Buenos Aires y La Plata, que distan entre sí 50 km, están ubicadas sobre terrenos llanos cuyos estratos de varios centenares de metros de profundidad son los de la llanura pampeana argentina (Russo y otros, 1979). Además, los $50 \mathrm{~km}$ de distancia entre ellas es bastante menor que los $1300 \mathrm{~km}$ de distancia epicentral de este sismo a la ciudad de La Plata y por ello debe ser pequeña la diferencia de la atenuación de las amplitudes de las distintas ondas sísmicas que integran el acelerograma.

b) En lo que respecta al comportamiento dinámico de los edificios analizados se supondrá que:

1. Como los movimientos de los edificios fueron relativamente pequeños, las propiedades dinámicas de los edificios altos durante este terremoto fueron similares a las que tenían cuando sus microvibraciones ambientales fueron registradas. En consecuencia, los valores de los períodos fundamentales de vibración son los detallados en la Tabla 8.3 e incluídos en la Fig. 8.3.

2. Los valores de amortiguación modal de los primeros modos de vibración de cada edificio serían no mayores del $2 \%$. Este valor, e incluso menores aún a $1.5 \%$, fueron obtenidos en los análisis de las microvibraciones ambientales registradas en los edificios altos aquí estudiados y también en otros estudios de vibraciones ambientales de edificios (Carmona y Herrera, 1969).

En consecuencia, las aceleraciones máximas absolutas de los movimientos en los niveles superiores de los edificios de Buenos Aires durante el sismo de Chile del 15 de Octubre de 1997 (UTC) alcanzaron valores de 7 y 9,5 gals para aquellos edificios con período de vibración fundamental de 1,2 y 2,7 seg, respectivamente, y un mínimo cercano a 2 gals para edificios con períodos comprendidos entre estos.

Estos valores de aceleración son 9 y 11 veces mayores que el máximo de la componente de aceleración en el terreno y ponen en evidencia la gran influencia del comportamiento dinámico del edificio en el movimiento resultante al ocurrir sismos con epicentros distantes y de gran magnitud. 
Esta notable amplificación es el resultado del contenido de frecuencias y duración del acelerograma, por una parte, y de los períodos de vibración y baja amortiguación de los edificios de mayor altura de la ciudad de Buenos Aires.

\section{5 - Resultados y discusión}

El evento chileno del 15 de Octubre de 1997 (UTC) tuvo una magnitud Mw = 7,1 y su epicentro fue localizado a $1320 \mathrm{~km}$ de la ciudad de Buenos Aires. Este valor de magnitud es menor que el de otros eventos sísmicos con su epicentro también en territorio chileno, que fueron sentidos con más intensidad en los edificios altos de Buenos Aires y aún levemente a nivel de terreno; también ha habido otros sismos con magnitud similar o levemente mayor a éste, con epicentros en la parte oeste de Argentina con distancias del orden de $1000 \mathrm{~km}$, que se han sentido con más intensidad en esta ciudad. No obstante lo mencionado, este evento ha permitido caracterizar por primera vez los movimientos en los edificios altos de la ciudad de Buenos Aires.

Se ha encontrado que los mayores valores en las curvas de respuesta de la aceleración absoluta (Fig. 8.7), de las componentes del acelerograma registrado en la ciudad de La Plata durante el sismo del 15 de octubre de 1997 (Fig. 8.4), están dentro del intervalo de los períodos fundamentales de los edificios altos en Buenos Aires detallados en la Tabla 8.3. Queda evidenciado entonces, que las mayores amplitudes del movimiento de estos edificios son generadas por las ondas sísmicas cuyos períodos son cercanos a 1,2 y 2,7 segundos.

En la Fig. 8.6 el máximo de 1,2 seg aparece tanto en las componentes horizontales como en la vertical, mientras que el máximo de 2,7 seg sólo se presenta en las curvas de las componentes horizontales, siendo mayor en la dirección norte-sur. De esto se deduce que estas ondas están horizontalmente polarizadas en la dirección perpendicular a la dirección de propagación.

Este análisis permite concluir que las mayores amplitudes del movimiento en los edificios considerados son generadas por las ondas volumétricas y por las ondas superficiales Lg.

Una medida de la potencia de las ondas Lg contenida en un acelerograma es la evaluación de la magnitud $\mathrm{mb}(\mathrm{Lg})$ por medio de la definición dada por Nuttli para la zona central de América del Norte (Nuttli, 1973):

$$
m b(L g)=\log A(D)+m b(D)+m b(0)
$$


donde: $A(D)$ es la amplitud de las ondas registradas a una distancia epicentral $D$, mientras que el segundo término representa la influencia de la atenuación debido a la distancia $\mathrm{D}$ en las amplitudes de ondas $\mathrm{Lg}$ y $\mathrm{mb}(0)$ es el valor de referencia.

Tal como para la magnitud $\mathrm{M}_{\mathrm{L}}$ y las magnitudes obtenidas a partir de las ondas volumétricas, esta magnitud Lg también presenta la saturación de su valor en relación al incremento del tamaño del evento sísmico, particularidad que comenzaría con $\mathrm{Mw}=6,6$ a 6,8 y estaría totalmente desarrollada para $\mathrm{Mw}=7,3$ a 7,6. Por ello, si la magnitud de saturación está presente en la evaluación de $\mathrm{mb}(\mathrm{Lg})$ a partir del registro instrumental obtenido en la ciudad de Buenos Aires o en La Plata, en otros sismos con igual distancia epicentral D que el del 15 de Octubre de 1997 pero con mayor magnitud que $M w=7,1$ sólo se incrementaría el término log $A(D)$ en un valor no mayor que $0,5 \mathrm{y}$, en tal caso, la amplitud $A(D)$ se incrementaría al triple para sismos chilenos de mayor magnitud que el del 15 de octubre de 1997.

De igual modo, si un sismo de mayor magnitud que el del 15 de octubre de 1997 ocurriese a menor distancia epicentral de Buenos Aireas y saturara la magnitud $\mathrm{mb}(\mathrm{Lg})$, el valor del término log $A(D)$ podría ser incrementado en la misma cantidad en que el segundo término decreciera. Si se emplea para la atenuación de las ondas Lg los valores obtenidos para la zona central de América del Norte (Nuttli, 1973), el decrecimiento del valor del segundo término sería cercano a 0,2 y por ello el incremento total como consecuencia de ambos efectos, que son los correspondientes a la saturación de la magnitud y la menor distancia epicentral, sería de 0,6 para $\log A(D)$, de donde $A(D)$ se incrementaría en casi cuatro veces la aceleración máxima del registro de La Plata obtenido en este análisis.

Otro importante factor a considerar en el efecto que ocasionan los sismos lejanos de magnitud mayor que $\mathrm{Mw}=8$ es el incremento en la duración (ya importante para el sismo de 1997) de la envolvente del desarrollo temporal de la aceleración en Buenos Aires, como una consecuencia del incremento del tiempo de ruptura en la fuente y de la subsiguiente dispersión de las ondas sísmicas así generadas. El aumento en la duración del movimiento percibido quizás sea el efecto más desagradable para los ocupantes de los niveles superiores de los edificios.

Por todo lo anteriormente expuesto se puede destacar:

1. Los valores máximos de aceleración del registro de la Fig. 8.4 son menores a un milésimo de la aceleración de la gravedad, niveles que no son suficientes para ser percibidos por la población a nivel de terreno. Por otra parte, el registro de 
aceleración muestra una relativamente larga duración de la parte significativa que es de casi 2 minutos.

2. En las curvas de respuesta de aceleración absoluta del citado acelerograma se distinguen dos máximos distintos, uno correspondiente al valor del periodo 1,2seg y otro al periodo 2,7seg (Fig. 8.5), con valores de aceleración absoluta para la amortiguación del $2 \%$, que son 6 y 8 veces las aceleraciones máximas registradas a nivel de terreno.

3. Estos períodos, para los que se presentan los valores máximos de la aceleración absoluta, están dentro del intervalo de los periodos fundamentales de vibración de los edificios mas altos de la ciudad de Buenos Aires detallados en la Tabla 8.3, por ejemplo, 2,3 y 2,7seg para el edificio Torre Le Parc que es el más alto con 160m.

4. Teniendo en cuenta la baja cantidad de amortiguación y las particularidades de las formas modales fundamentales de estos edificios, la aceleración absoluta de los movimientos en sus niveles superiores son hasta 10 veces mayor que la máxima aceleración del terreno durante estos sismos lejanos y de gran magnitud.

5. Para otros sismos que ocurriesen con área epicentral en Chile y con mayor magnitud que éste de octubre de 1997, de $M s=8$, las aceleraciones a nivel de terreno en la ciudad de Buenos Aires se incrementarían a no menos del triple de las máximas registradas en 1997 (Fig. 8.4) y este incremento podría ser de cuatro veces para sismos mayores con área epicentral en el oeste argentino; alcanzarían así valores de aceleración en el terreno de casi 4 milésimos de la gravedad, nivel que puede ser percibido ya por algunas personas. Para estos sismos lejanos de gran magnitud, se incrementaría notablemente la duración de la parte significativa del movimiento.

6. En el caso de ocurrencia de estos sismos lejanos de gran magnitud, por la notable amplificación que se produce en los niveles superiores de los edificios más altos de la ciudad de Buenos Aires, la aceleración absoluta del movimiento en tales niveles alcanzaría a cerca de 30 gals, aproximadamente $0,03 \mathrm{~g}$, y la duración de la percepción alcanzaría varios minutos. En tal caso, los ocupantes de los niveles superiores de estos edificios percibirían durante algunos minutos un movimiento muy desagradable que indudablemente generaría amplia alarma en las personas y en algunos casos pánico de difícil control, tal como ocurrió durante el sismo de Caucete, San Juan, del 23 de noviembre de 1977. 


\section{CONCLUSIONES}

En este trabajo de Tesis de Doctorado presento los estudios y controles que llevé a cabo para implementar una red sismológica digital que provea datos de calidad y dentro de los estándares internacionales, aportando información que proviene de una región que anteriormente no se encontraba instrumentada (sudeste del territorio nacional). Además, presento dos aplicaciones en las cuales, a partir del procesamiento de datos digitales provistos fundamentalmente por la estación sismológica de La Plata, obtuve resultados acerca de la estructura interna de la corteza en las Sierras Pampeanas y de la respuesta de los edificios de mayor altura de la ciudad de Buenos Aires en ocasión de eventos de gran magnitud.

En la estación sismológica de La Plata, que desde el año 1962 funcionó con instrumental donado por el USGS y formó parte de la red sismológica WWSSN hasta el año 1996, llevé a cabo la actualización del sistema de adquisición en varias etapas, resultando en la actualidad una estación de banda ensanchada y con registro digital, que se agrega al sistema analógico tradicional que se conserva.

Además en el mismo recinto instalé, en el año 1997, acelerómetros que permitieron obtener registros con los cuales realicé los estudios de ruido y que muestran la calidad de los datos obtenidos en esta estación.

En la estación sismológica Trelew (TRWA), realicé la primer instalación temporaria en el año 1997 y luego, la instalación de instrumental permanente a partir del año 1999. En este sitio, los estudios de ruido indicaron la conveniencia de construir un recinto por debajo del nivel del suelo, obteniendo entonces una estación que cuenta actualmente con instrumental de banda ancha. Esta estación provee información con una buena relación señal-ruido, de acuerdo a los estándares internacionales, según lo que se desprende del estudio realizado.

En la estación sismológica Despedida (DSPA), en una primera etapa (año 1999) instalé un instrumento de corto período, previo a lo cual hice los estudios pertinentes. Con los datos obtenidos, hice un análisis de sismicidad que demostró la importancia de contar con una estación sismológica de última generación en ese sitio. La posibilidad de contar con registros de eventos de magnitudes menores permitirá, por ejemplo, proveer información de la geodinámica de esta región activa, en la que ha habido un sismo de mayor magnitud en el pasado. 
Desde fines del año 2002, se cuenta con instrumental digital de banda ancha que forma parte de una red ítalo-argentina, la que está previsto que en un futuro próximo forme parte de una red digital mundial.

Como conclusión, en esta primer etapa he instalado con éxito tres estaciones sismológicas digitales de banda ancha en la región este-sudeste de nuestro país, que brindan una mínima cobertura de esa zona. Estas estaciones proveen datos con relaciones señal-ruido que cumplen con los requisitos de calidad según los estándares internacionales.

El hecho de contar con datos sismológicos digitales de sismómetros y acelerómetros permite realizar diversas aplicaciones. En particular, en este trabajo presento dos aplicaciones con datos obtenidos fundamentalmente en La Plata.

- El análisis de ondas superficiales a través del cálculo de las curvas de dispersión realizado con la técnica de Filtrado Múltiple, aplicado por primera vez en nuestro país, me permitió obtener curvas de dispersión para distintas trayectorias en territorio argentino. Con este estudio comprobé que los datos de La Plata resultaron útiles para obtener curvas que reflejan claramente las distintas trayectorias entre evento y estación. A partir de la inversión de curvas de dispersión obtuve perfiles de velocidad de corteza y manto superior. Las trayectorias que atraviesan los Andes y zonas cratónicas, pueden considerarse de espesor cortical similar, aproximadamente $45-55 \mathrm{~km}$ pero con diferentes velocidades. En el caso de las trayectorias que atraviesan las Sierras Pampeanas, las velocidades de ondas transversales resultantes son bajas; esto corresponde a medios de carácter astenosférico, reflejo de la existencia de un área termal además de una anomalía eléctrica en la corteza profunda encontrada por otros métodos geofísicos. En las trayectorias que incluyen eventos cuyos caminos atraviesan sólo zonas cratónicas, los espesores corticales son menores, de aproximadamente $32 \mathrm{~km}$.

Este estudio aportó información basada en el análisis de velocidades de ondas superficiales a través del método de una única estación. Aún con las limitaciones propias del mismo, este trabajo es el primero para obtener estimaciones regionales del Moho a lo ancho de nuestro país y los modelos 1D 
obtenidos de la inversión de las curvas de dispersión están de acuerdo con resultados obtenidos con otros métodos geofísicos.

- $\quad$ El análisis de los acelerogramas de terremotos provenientes de la zona costera de Chile central (USGS-NEIC) de magnitud mayor, ha permitido caracterizar las propiedades dinámicas de los estratos de terreno sobre los que está ubicada la estación sismológica de registro. Teniendo en cuenta que la estación de La Plata se encuentra sobre un suelo blando de características similares al suelo en la ciudad de Buenos Aires, he podido caracterizar, por primera vez, la respuesta de edificios altos en dicha ciudad en ocasión de la ocurrencia de movimientos sísmicos que actúan como excitaciones dinámicas sobre los mismos. Los mayores valores en las curvas de respuesta de la aceleración absoluta de las componentes del acelerograma, registrado en la ciudad de La Plata durante el sismo del 15 de octubre de 1997, están dentro del intervalo de los periodos fundamentales de algunos edificios altos en Buenos Aires. Queda evidenciado entonces, que las mayores amplitudes del movimiento de estos edificios son generadas por las ondas sísmicas cuyos períodos son cercanos a 1,2 y 2,7 segundos y que en el caso de sismos de mayores magnitudes provenientes del litoral chileno, así como del oeste de nuestro país, en los pisos superiores de estos edificios se percibirán vibraciones.

Los resultados obtenidos demostraron que, aún cuando la ciudad de Buenos Aires está ubicada en una región de peligrosidad sísmica muy reducida, los efectos producidos por sismos de mayor magnitud, provenientes del litoral chileno o del oeste argentino, se percibirán con cierta intensidad en los edificios altos. Si bien esto no implicaría un riesgo estructural, las oscilaciones que sobrevendrían en los niveles superiores de esos edificios serán sentidos durante algunos minutos. Como conclusión, considero que sería conveniente realizar tareas de prevención con la población para evitar alarmas o escenas de pánico de difícil control. 


\section{REFERENCIAS}

Aki, K. y P. G. Richards, 1980. Quantitative Seismology-theory and methods. W. H. Freeman and Company, San Francisco.

Astiz , L., 1997. Characteristic low and high noise models from robust PSE of seismic noise of broadband stations, presented at IASPEI 1997 meeting.

Beck, S. L., Zandt, G., Myers, S. C., Wallace, T. C., Silver, P. G. y Drake, L., 1996. Crustal thickness variations in the Central Andes. Geology, 24, 407-410.

Bormann, P. (editor), 2002. New manual of seismological observatory practice (NMSOP), GeoForchungsZentrum Postdam.

Cahill , T. y Isacks B. ,1992. Seismicity and shape of the subducted Nazca Plate. J. Geophys. Res., 97, 17503-17529.

Carmona, J.S. y Herrera J., 1969. "Periods of Buildings of Mendoza City (Argentina)", Proceedings IV World Conference on Earthquake Engineering, Santiago, Chile.

Carmona, J. S. y otros., 1978. "El Sismo de Caucete, San Juan, Argentina, del 23 de noviembre de 1977 y la Seguridad que Proveen las Normas Sismorresistentes", Proceedings Seminario Internacional de Preparación para Atención de Catástrofes - Viña del Mar, Chile.

Carmona, J. S., Sisterna, C. A. y Magrini, M.C., 1995. "Periodos de Vibración Medidos en Edificios Torre de la Ciudad de Buenos Aires con Altura Mayor de 100 m.", Proceedings XXVII Jornadas Sudamericanas de Ingeniería Estructural, Tucumán, Argentina, Vol. 6, pp 561-571.

Carmona J., Sabbione N., Sisterna C., Pinciroli R., Magrini M., Palau, R., Plasencia M., y García L., 2000. El movimiento causado por los grandes sismos distantes en los edificios mas altos de Buenos Aires a partir de la medida del efecto del sismo de Chile del 14-X-1997 - Congreso de Ingeniería 2000. . Contr. Tecn. AIE, pp. 433-446. Con ref.

Chebli, Walter, C. Nakayama y J. Schiutto, 1978. Mapa geológico de la provincia del Chubut. VII Congreso Geológico Argentino. Actas I, pp 639-655.

Cunningham, W.D., 1993. Strike-slip faults in the southernmost Andes and the development of the Patagonian orocline. Tectonics, 12(1): 169-186.

Cunningham, D.W., Dalziel, I.A.W., Tung-Yi, L. y Lawver, L.A., 1995. Southernmost South America-Antarctic Peninsula relative plate motions since $84 \mathrm{Ma}$ : implications for the tectonic evolution of the Scotia Arc region. Journal of Geophysical Research 100 (B5): 8257-8266.

Davis, G.H., Bump, A.P., García, P.E. y Ahlgren, S.G., 2000. Conjugate Riedel deformation band shear zone. Journal of Structural Geology 22(2): 169-190.

Dorbath, C., Granet, M., Poupinet, G. y Martinez, C., 1993. A Teleseismic Study of the Altiplano and the Eastern Cordillera in Northern Bolivia: New Constraints on a Lithospheric Model. J. Geophys. Res., 98, 9825-9844.

Dziewonski, A., Bloch, S. y Landisman, M., 1969. A technique for the analysis of transient seismic signals. Bull. Seis. Soc. Am. 59, 427-444.

Febrer, J.M., 2002. La red ASAIN de observación sismológica en Antártica, II. Sismicidad en Tierra del Fuego. CD XXI Reunión de la Asociación Argentina de Geofísicos y Geodestas, pp,273-279.

Götze, H. J., Lahmeyer, B., Schmidt, S. y Strunk, S., 1994. The lithospheric structure of the Central Andes (20-25) as inferred from quantitative interpretation of regional gravity, in Tectonics of the Southern central Andes: structure and evolution of an active continental margin, edited by K. J. Reutter, E. Scheuber, and P. J. Wigger, pp. 23-48, Springer-Verlag, Berlin.

Gershanik, S., 1996. Sismología. Universidad Nacional de La Plata. 
Havskov J. y G. Alguacil, 2002. Instrumentation in Earthquake Seismology . University of Bergen and University of Granada. Preliminary version, pp. 305

Introcaso, A., Robles, A. y Guspi, F., 1990. Interpretación gravimétrica preliminar en la cuenca del Bermejo. Geoacta, 17(2), 179-202.

James, D. y Snoke, J., 1994. Structure and tectonic in the region of flat subduction beneath Central Peru: Crust and uppermost mantle. J. Geophys. Res., 99, 6899-6912.

Jaschek, E.,1972 ¿Hay sismos en la Provincia de Buenos Aires?. Ciencia e Investigación. Tomo 28 Nro. 1, pp.26-29.

Jaschek, E., N. Sabbione y P. Sierra, 1982. Reubicación de Sismos localizados en Territorio Argentino (1920 - 1963). Observatorio Astronómico de la Universidad Nacional de La Plata. Serie Geofísica, Tomo XI, № 1.

Jordan, T., Isacks, B., Allmendinger, J., Brewer, J., Ramos, V. y Ando, C., 1983. Andean tectonics related to geometry of subducted Nazca Plate. Geol. Soc. Am. Bull., 94, 341-361.

Kocaoglu, A. H. y Long, L. T., 1993. A review of time-frequency analysis techniques for estimation of group velocities. Seism. Res. Lett., 64, 157-167.

Kushnir, A. F., Levshin, A. L. y Lokshtanov, D. E., 1988. Determination of the velocity section from the spectra of surface waves by nonlinear optimization methods. Problems of Seismological Information Science, Comput. Seismology, 21, Allerton Press, NY, 137-152.

Lay, T. y R. Wallace, 1995. Modern Global Seismology. Academic Press, pp. 497

Lee, W.H.K., 1989. Toolbox for seismic data acquisition, processing and analysis. IASPEI software library, Vol I. Published by IASPEI in collaboration with Seismological Society of America.

Lesta, Pedro, R. Ferello y G. Chebli, 1976. Chubut extraandino. $2^{\circ}$ Simposio de Geología Regional Argentina. Academia Nacional de Cs. de Córdoba. Vol II, pp 1307-1387.

Levshin, A. L., Pisarenko, V. F. y Pogrebinsky, G.A., 1972. On a frequency-time analysis if oscillations. Ann. Geophys., 28, 211-218.

Lokshtanov, D. E, 1993. UDIMA program. Personal communication.

Mooney, W., Laske, G. y Guy Masters, T., 1998. CRUST 5.1: a global crustal model at $5^{\circ} \times 5^{\circ}$. J. Geophys. Res., 103, 727-747.

Lomnitz, C., 1970. Major Earthquakes and Tsunamis in Chile, Geologische Rundschau, Band 59, pp. 951,955, Springer Verlag, Stuttgart.

Lomnitz, C.,1988. Las causas probables de la catástrofe sísmica del 19 de septiembre de 1985. www.ssn.unam.mx/SSN/DOC/CONACYT/88-82cinna.htm

Longuet-Higgins, M. S, 1950. A theory of the origin of microseisms. Philosophical Transactions of the Royal Society of London A243: 1-35.

Lorca Mella, Emilio y M, Recabarren Herrera, 2003: Terremotos y tsunamis o maremotos, cap, 5, (ed,) Servicio hidrográfico y oceanográfico de la armada de Chile, Comisión oceanográfica intergubernamental, Centro integral de información de tsunamis, pp 127,

Mooney, W., Laske, G. y Guy Masters, T., 1998. CRUST 5.1: a global crustal model at $5^{\circ}$ x 5․ J. Geophys. Res., 103, 727-747.

.Nuttli, O.W.,1973. Seismic wave attenuation and magnitude relations for eastern North America, J. Geophys. Res.,78, pp876-885.

Olivero E., Malagnino E.y Gagliardini D. 1995. Interpretación Preliminar del Sistema de Fracturas del Este de Tierra del Fuego basada en Imágenes ERS-1. SELPER, Rev. Técnica de Integración Iberoamericana y Mundial. Vol. 11, Nro. 1-2. Pag 34-39. 
Olivero, E., 1997. Jurassic-Paleogene Stratigraphy and Basin Evolution of the Eastern Andes of Tierra del Fuego. GAEA heidelbergensis: 258-259, IAS $18^{\text {th }}$ European Regional Meeting of Sedimentology, Heidelberg, Alemania.

Olivero, E. B. y Martinioni, D., 2001. A review of the geology of the Argentinian Fuegian Andes, J. Soc. Am. Earth. Sci, 14, 175-188.

Osella, A. M., Pomposiello C., Maidana, A. y Borzotta, E., 1992. Electromagnetic Studies in the Tucumán Plain. Acta Geod. Geophys. et Mont., 27(1): 103-110

Osella, A. M., Pomposiello, C., Favetto, A., Sainato, C. y Martinelli, P., 1993. Bidimensional modeling of a geothermal anomaly in the eastern border of Aconquija range. Acta Geod. Geophys. Mont., 28, 329-341.

Peterson, J., C. Hutt y L. Holcomb, 1980. Test and Calibration of the Seismic Research Observatory. United States Department of the Interior. Geological Survey. Open File Report 80-187, pp. 89.

Peterson, J., 1993. Observations and modeling of seismic background noise. U.S.Geol. Survey Open-File Report 93-322, 95 pp.

Pinciroli, R., Sabbione, N. y Rosa, M. L., 1997. Aspectos del ruido sísmico en la estación LPA. Actas 19a Reunión Científica de Geofísica y Geodesia, 237-241. Con Ref..

Pinciroli R., Sabbione N., Rastelli C.y Pinciroli E., 2000. Sensores de banda ancha en la estación sismológica de Trelew. Actas de la 20va. Reunión Científica de la Asociación Argentina de Geofísicos y Geodestas. En CD, pp. 96-102. Con ref.

Plasencia, G. Connon, J. L. Hormaechea y N. Sabbione, 2002. Determinación preliminar de epicentros registrados en la estación sismológica DSPA (Despedida), prov. de Tierra del Fuego.M. CD XXI Reunión Científica Asociación Argentina de Geofísicos y Geodestas. 23-27, pp 285-289.

Pomposiello C., Osella, A.M., Maidana, A. y Borzotta, E., 1994. MT studies in SouthEast region of Tucumán Plain in NW Argentina. Journal of South-American Earth Sciences, 7(2), 101-107.

Pomposiello C., Osella, A.M., Favetto, A., Sainato, C., Martinelli, P. y Aprea, C., 1998. Current channelling and three-dimensional effects detected from magnetotelluric data from a sedimentary basin in Sierras Pampeanas, Argentina. Geophys. J. Inter., 135 (2), 339-351.

Pomposiello, M. C., Favetto, A., Sainato, C., Booker, J. y Li, S., 2002. Imaging the sedimentary basin of the Tucumán Plain in the northern Pampean Ranges, Argentina. J. Applied Geoph., Special Issue, 47-58.

Rastelli, C., N. Sabbione y R. Pinciroli, 1997. Registración sismológica en Trelew, julio - Octubre 1997. Informe Técnico biblioteca FCAG-UNLP.

Regnier, M., Chatelain. J., Smalley, R. Jr., Chiu J., Isacks, B. y Araujo, M., 1992. Seismotectonics of Sierra Pie de Palo, a basement block uplift in the Andean Foreland of Argentina. Bull. Seis. Soc. Am. 82, pp. $2549-2571$.

Regnier, M., Chiu, J., Smalley, R. Jr., Isacks, B. y Araujo, M., 1994. Crustal thickness variation in the Andean foreland, Argentina, from converted waves. Bull. Seis. Soc. Am., 84, 1097-1111.

Rial, J. y Ritzwoller, M., 1997. Propagation efficiency of long-period Lg waves in the South American continent. Geophys. J. Int., 131, 401-408.

Roberts R. G., Christofferson A. y Cassidy F., 1989. Real-time detection, phase identification and source location estimation using single station threecomponent seismic data. Geophys. J. R. Astr. Soc., 97, 471-480.

Rosello, E., Mozetic, M., Cobbold, P., de Urreiztieta, M., Gapais, D. y Lopez Gamundi, O., 1996. The Valle Fertil flower structure and its relationnship with the Precordillera and Pampean ranges. Third ISAG, 481-484. 
Russi M. y Febrer J.M., 2001, Broad-band seismology in the Scotia Sea region, Antarctica, Italian and Argentinean contributions to the Scotia Sea Broadband Network, In "Results from the Workshop Broadband Seismic Observations and the Goedynamics of the Scotia Sea Region", Trieste, Italy, 25-26 octubre 1999. Giuliano Brancolini, Giuliano F. Panza, Marino Russi \& Francias Wu editors, Terra Antartica, 2001, 8(2), 29-34.

Russo, A, y otros., 1979. Llanura Chaco Pampeana, Proceedings Segundo Simposio de Geología Regional Argentina, Academia Nacional de Ciencias, Córdoba, Argentina, Vol. I, pp139-183.

Sabbione N., Pinciroli R., Rastelli C. y Roizman M., 1995. Procesamiento de datos sismológicos digitales en La Plata. Cuartas Jornadas Geológicas y Geofísicas Bonaerenses 2, 161-168. Con Ref.

Sabbione N., Roizman M., Rastelli C. y Plasencia ,M., 1997. Curvas de dispersión de ondas Rayleigh en distintas trayectorias hacia la La Plata (Pcia. Bs. As.). Actas 19a Reunión Científica de Geofísica y Geodesia, pp. 253-257.

Sabbione, N., Rastelli, C., Pinciroli, R. y Plasencia, M., 1998. Análisis comparativo de datos sismológicos obtenidos en Trelew (Pcia de Chubut) y La Plata (Pcia. de Buenos Aires). Actas de las V Jornadas Geológicas y Geofísicas Bonaerenses. Vol 2, pp. 209-216. Con ref.

Sabbione, N., R. Pinciroli, C. Rastelli, M. Plasencia y G. Connon, 2000a. Estaciones sismológicas en Trelew y Río Grande, Argentina. Actas de la 20va. Reunión Científica de la Asociación Argentina de Geofísicos y Geodestas. C.A. En CD, pp. X-XV. Con ref.

Sabbione N., Carmona J., Pinciroli R. y Gregori D., 2000b. Las frecuencias dominantes de acelerogramas registrados en La Plata por efectos de sismos de mayor magnitud del centro de Chile. Actas de la 20va. Reunión Científica de la Asociación Argentina de Geofísicos y Geodestas. En CD, pp. 103-108. Con ref.

Sabbione, N., Pinciroli, R., Rastelli, C., Plasencia M. y Connon G., 2001. New Seismological Stations in Trelew and Rio Grande, Argentine. (Results from the Workshop on Broad-Band Seismic Observations and the Geodynamics of the Scotia Sea Region, Antarctica. Trieste). Terra Antarctica. Vol 8 (2), 111-114.

Sabbione, N., M. L. Rosa y A. M. Osella, 2003. Analisis preliminar de ondas superficiales aplicado al modelado cortical en las Sierras Pampeanas, Argentina. Geoacta, vol. 28. pp. 49-60

Sabbione, Nora, 2004. Red de estaciones sismológicas digitales en la Universidad Nacional de La Plata. Su puesta en funcionamiento. Capítulo de Libro Tópicos de geociencias. Un volumen de estudios sismológicos, geodésicos y geológicos en homenaje al Ing. Fernando Séptimo Volponi. Editorial EFU, Fundación Universidad Nacional de San Juan, pp. 95-138.

Schawrz, G., Chong, G., Kruger, D., Martinez, E., Massow, W., Rath, V. y Viramonte, J., 1994. Crustal high conductivity zones in the southern Central Andes, in Tectonics of the Southern central Andes: structure and evolution of an active continental margin, edited by K. J. Reutter, E. Scheuber and P. J. Wigger, pp. 49-67, Springer-Verlag, Berlin.

Scherbaum F. y Johnson J., 1993. PITSA, Programmable Interactive Toolbox for Seismological Analysis. IASPEI Software Library, Vol. 5.

Shearer, P. M.,1999. Introduction to Seismology. Cambridge University Press, pp. 260. SeisAn, 2003: The Earthquake analysis software, Institute of Solid Earth Physics, Bergen University, Norway. (http://www.ifjf.uib.no). 
Smalley, R., Pujol, J., Regnier, M., Chiu, J., Chatelain, B. y Puebla, N., 1993. Basement seismicity beneath the Andean Precordillera thin-skinned thust belt and its implications for crustal and lithosphere behavior. Tectonics, 112, 63-76.

Snoke, J. y James, D. 1997. Lithosferic structure of the Chaco and Paraná basins of South America from surface-wave inversion. J Geophys. Res., 102, 2939-2951.

Stipanicic, Pedro y E. Methol, 1976. Comarca Norpatagónica. $2^{\circ}$ Simposio de Geología Regional Argentina. Academia Nacional de Cs. de Córdoba. Vol II, pp 10711097.

Tapley, W.C. y J.E.Tull, 2000. SAC2000, Seismic Analysis Code,. Lawrence Livermore National Laboratory. (http://www-ep.es.Ilnl.gov/tvp/sac.html).

Toselli, A., Pomposiello, C., Durand, F., Osella, A. y Lopez de Luchi, M., 1999. South American Global Geoscience Transect 7: Cordillera Frontal to Llanura Chaco Pampeana, Northern Argentina. International Geology Review, 41, 154-174.

Uhrammer, R.A., W. Karavas y B. Romanowiczs, 1998. "Broadband Seismic Station Installation Guidlines” Seismological Reserch Letters Vol.69 № 1, pp15-26.

Whitman, D., 1994. Moho geometry beneath the eastern margin of the Andes, northwest Argentina, and its implications to the effective elastic thickness of the Andean foreland . J. Geophys. Res., 99, 15277-15289.

Zandt, G., Velasco, A. y Beck, S. L., 1994. Composition and thickness of the southern Altiplano crust, Bolivia. Geology, 22, 1003-1006. 